\title{
Coordination environment-controlled photoinduced electron transfer quenching in luminescent europium complexes
}

Daniel Kovacs, Emilie Mathieu, Salauat R. Kiraev, Jordann A. L. Wells, Ellen Demeyere, Agnès Sipos, and K. Eszter Borbas *

Department of Chemistry, Ångström Laboratory, Box 523, Uppsala University, 75120, Uppsala, Sweden

Email: eszter.borbas@kemi.uu.se

$\begin{array}{ll}\text { General procedures } & \mathrm{S} 2\end{array}$

Paramagnetic ${ }^{1} \mathrm{H}$ NMR $\quad$ S2

$\begin{array}{ll}\text { Chromatography } & \text { S2 }\end{array}$

Electrochemistry S3

$\mathrm{UV}-\mathrm{Vis}$ absorption and emission spectroscopy $\quad \mathrm{S} 4$

Crystallography S6

$\begin{array}{ll}\text { Synthesis and chemical characterization } & \text { S9 }\end{array}$

$\begin{array}{lr}{ }^{1} \mathrm{H} \text { NMR spectra of Eu(III) complexes } & \text { S30 }\end{array}$

$\begin{array}{ll}X \text {-ray diffraction data } & \text { S35 }\end{array}$

$\begin{array}{ll}\text { Cyclic voltammograms } & \text { S40 }\end{array}$

$\begin{array}{ll}\text { Photophysical characterization } & \text { S53 }\end{array}$

${ }^{1} \mathrm{H},{ }^{13} \mathrm{C}$ and ${ }^{19} \mathrm{~F}$ NMR spectra $\quad$ S84

$\begin{array}{ll}\text { References } & \text { S131 }\end{array}$ 
General Procedures. ${ }^{1} \mathrm{H}$ NMR (400 MHz), ${ }^{13} \mathrm{C}$ NMR (100 MHz) and ${ }^{19} \mathrm{~F}$ NMR (376 MHz) spectra were recorded on a JEOL $400 \mathrm{MHz}$ instrument. Chemical shifts were referenced to residual solvent peaks and are given as follows: chemical shift $(\delta, \mathrm{ppm})$, multiplicity (s, singlet; br, broad; d, doublet, t, triplet; q, quartet; m, multiplet), coupling constant (Hz), integration. LC-MS analysis was carried out using an analytical Dionex UltiMate 3000 HPLC instrument coupled to a Thermo Finnigan LCQ DECA XP MAX mass spectrometer. HR-ESI-MS analyses were performed at the Organisch Chemisches Institut WWU Münster, Germany. All compounds displayed the expected isotope distribution pattern. Anhydrous $\mathrm{CH}_{2} \mathrm{Cl}_{2}$ was obtained by distillation from $\mathrm{CaH}_{2}$ under an Ar atmosphere.

Compounds $\mathbf{1},{ }^{1} \mathbf{6}^{2}{ }^{1} 1^{\mathrm{Me}},{ }^{3}$ and $\mathbf{1 1}^{\mathrm{CF} 3[3]}$ were synthesized following literature methods. All other chemicals were from commercial sources and used as received.

Paramagnetic ${ }^{1} \mathrm{H}$ NMR. ${ }^{1} \mathrm{H}$ NMR spectra of Eu-complexes were recorded at 400 $\mathrm{MHz}$ using the following parameters: cooling for 5 min until the temperature stabilizes at $0 \pm 0.1^{\circ} \mathrm{C}$ for samples measured in $\mathrm{CD}_{3} \mathrm{OD}$ and at $10 \pm 0.1^{\circ} \mathrm{C}$ for samples measured in $\mathrm{D}_{2} \mathrm{O}$; relaxation delay: $1 \mathrm{~s}$; number of scans: 128; number of points: 131072 ; range: -60 to $60 \mathrm{ppm}$.

Chromatography. Preparative chromatography was carried out on silica gel [Normasil 60 chromatographic silica media (40-63 micron)] and aluminum oxide [activated, neutral, Brockmann Activity I, Sigma-Aldrich]. Thin layer chromatography was performed on silica-coated (60 F254) aluminum plates from Merck and aluminum oxide coated with $254 \mathrm{~nm}$ fluorescent indicator aluminum plates from Sigma-Aldrich. Samples were visualized by UV-light (254 and $365 \mathrm{~nm}$ ).

HPLC-analysis was performed on a Dionex UltiMate 3000 system using a Phenomenex Gemini® ${ }^{\circledR}$ C18 TMS end-capped $150 \mathrm{~mm} \times 4.6 \mathrm{~mm}$ HPLC column with water $\left(0.05 \%\right.$ formic acid): $\mathrm{CH}_{3} \mathrm{CN}$ ( $0.05 \%$ formic acid) eluent system using the 
methods: (a) $0-10 \mathrm{~min}: 10 \% \rightarrow 90 \% \mathrm{CH}_{3} \mathrm{CN}, 0.5 \mathrm{~mL} / \mathrm{min}$; (b) $0-12 \mathrm{~min}: 10 \% \rightarrow 50 \%$ $\mathrm{CH}_{3} \mathrm{CN} \& 12-14 \mathrm{~min}: 50 \% \rightarrow 90 \%, 0.5 \mathrm{~mL} / \mathrm{min}$; (c) $0-8 \mathrm{~min}: 10 \rightarrow 20 \%$ \& $8-12 \mathrm{~min}:$ $20 \%$ iso $\mathrm{CH}_{3} \mathrm{CN}, 0.5 \mathrm{~mL} / \mathrm{min}$; (d) $0-6 \mathrm{~min}: 10 \%$ iso $\mathrm{CH}_{3} \mathrm{CN} \& 6-12 \mathrm{~min}: 10 \% \rightarrow 50 \%$, $0.25 \mathrm{~mL} / \mathrm{min}$. UV - (UltiMate 3000 Photodiode Array Detector) and ESI-MS detections (LCQ DECA XP MAX) were used.

Electrochemistry. Cyclic voltammograms were obtained in an argon atmosphere at room temperature $\left(\sim 20^{\circ} \mathrm{C}\right)$ using an AUTOLAB PGSTAT 100 potentiostat, or an AUTOLAB PGSTAT $204 \mathrm{~N}$ potentiostat, equipped with a $3 \mathrm{~mm}$ glassy carbon electrode, a platinum wire auxiliary electrode, and a saturated calomel reference electrode. The solution was stirred in between each measurement. The solution was let to equilibrate for $10 \mathrm{~s}$ at the start potential before starting the measurements. A step potential of $0.9 \mathrm{mV}$ was used. Measurements were performed in distilled water with $\mathrm{LiCl}(0.1 \mathrm{M})$ as the supporting electrolyte.

A solution of $\mathrm{LiCl}(0.1 \mathrm{M})$ was prepared and $\mathrm{pH}$ was set to $\sim 6.5$ by addition of $\mathrm{NaOH}(0.1$ M) or $\mathrm{HCl}(0.1 \mathrm{M})$. This solution was added to the electrochemical cell, allowed to stir, and purged with argon for 10 min prior to each measurement. The working electrode was polished with $0.05 \mu \mathrm{m}$ alumina on a polishing pad, washed with water and ethanol, and dried with air. The three electrodes (GC working electrode, platinum wire auxiliary electrode, and SCE reference electrode) were inserted into the cell setup and a background scan was recorded with a scan rate of $100 \mathrm{mV} / \mathrm{s}$, and four sweeps. A lack of oxygen redox signal verified that oxygen had been removed below detectable levels. The europium complex $(1 \mathrm{mM})$ was added in the solution, and the $\mathrm{pH}$ of the resulting solution was adjusted to $\sim 6.5$ (Table S5) by addition of $\mathrm{NaOH}(0.1 \mathrm{M})$ or $\mathrm{HCl}(0.1 \mathrm{M})$. The resulting solution was stirred and purged with argon for 10 min. Scans were recorded at various scan rates (50 to $1000 \mathrm{mV} / \mathrm{s}$ ) with four sweeps for each measurement. Three independent measurements were performed for each compound. The voltammograms obtained at various scan rates are shown in Figures S15-25. The current 
intensities were plotted $v s$. the square root of scan rate and fit to a linear regression to ensure that the electron transfer was heterogenous.

UV-Vis absorption and emission spectroscopy. All measurements were performed in PIPES buffered distilled water at $\mathrm{pH}$ 6.5. Complex concentration was nominally 10 $\mu \mathrm{M}$, however, small quantities of Ln salts may diminish this. Glycerol was of $99.9+\%$ purity. Quartz cells with $1 \mathrm{~cm}$ or $0.2 \mathrm{~cm}$ optical pathlengths were used for the room temperature measurements. The absorbance spectra were measured by a Varian Cary 100 Bio UV-Visible spectrophotometer. The emission and excitation spectra, lifetimes, time-resolved spectra and quantum yields were recorded on a Horiba FluoroMax-4P. All emissions were corrected by the wavelength sensitivity (correction function) of the spectrometer. All measurements were performed at room temperature unless stated otherwise.

Quantum yields were measured at room temperature, using quinine sulfate (QS) in $\mathrm{H}_{2} \mathrm{SO}_{4} 0.05 \mathrm{M}\left(\Phi_{\text {ref }}=0.59\right)$ as reference, ${ }^{4}$ in Equation (1). Quantum yields were calculated according to (1), with $\Phi_{\mathrm{s}}$ the quantum yield of the sample, $\Phi_{\text {ref }}$ the quantum yield of the reference, $I$ the integrated corrected emission intensity of the sample (s) and of the reference (ref), $f_{\mathrm{A}}$ the absorption factor of the sample (s) and of the reference (ref) at the excitation wavelength and $n$ the refractive indexes of the sample (s) and of the reference (ref). The concentration of the complexes was adjusted to obtain an absorbance around the maxima of the antennae matching that of the QS fluorescence standard. The excitation wavelength where the absorption factors of the samples and of the reference were the same was chosen (i.e. where the absorptions are identical). The corrected emission spectra of the sample and reference standard were then measured under the same conditions over the $330-800 \mathrm{~nm}$ spectral range as well as blank samples containing only the solvent (i.e. PIPES buffered aqueous solutions). The appropriate blanks were 
subtracted from their respective spectra, and the antenna fluorescence and lanthanide luminescence were separated by fitting the section of the antenna emission overlapping the lanthanide emission with an exponential decay or with a scaled emission spectrum from the corresponding gadolinium complexes. The quantum yields were then calculated according to (1). The given relative error on the quantum yields $(\delta \Phi=\Delta \Phi / \Phi$, where $\Delta \Phi$ is the absolute error) take into account the accuracy of the spectrometer and of the integration procedure $\left[\delta\left(I_{s} / I_{r e f}\right)<2 \%\right]$, an error of $0.59 \pm 0.01$ on the quantum yield of the reference QS $\left[\delta\left(\Phi_{\text {ref }}\right)<2 \%\right]$, an error on the ratio of the absorption factors $\left[\delta\left(f_{\text {Aref }} / f_{A s}\right)\right.$ $<5 \%$, relative to the fixed absorption factor of the reference QS] and an error on the ratio of the squared refractive indexes $\left[\delta\left(n_{s}^{2} / n_{r e f}^{2}\right)<1 \%,<0.25 \%\right.$ around 1.333 on each individual refractive index], which sums to a total estimated relative error that should be $\delta \Phi_{s}<10 \%$. A limit value of $10 \%$ is thus chosen.

$$
\Phi_{s}=\frac{I_{S}}{I_{\text {ref }}} \cdot \frac{f_{\text {Aref }}}{f_{A s}} \cdot \frac{\left(n_{S}\right)^{2}}{\left(n_{\text {ref }}\right)^{2}} \cdot \Phi_{\text {ref }}
$$

Low temperature measurements were done in quartz capillaries at $77 \mathrm{~K}$ by immersion in a liquid $\mathrm{N}_{2}$-filled quartz Dewar and with addition of glycerol (1 drop) to the solutions (9 drops) measured at room temperature.

Lifetimes in the millisecond range were recorded $0.05 \mathrm{~ms}$ after pulsed excitation at the excitation maxima ( $\left.\lambda_{\mathrm{ex}}\right)$ between $300-345 \mathrm{~nm}$ by measuring the decay of the lanthanide main emission peak (i.e. Eu $615 \mathrm{~nm}$, Tb $545 \mathrm{~nm}$ ). The increments after the initial delay were adjusted between $0.2-20 \mu$ s depending on the lifetime in order to have a good sampling of the decay. The obtained data were fitted by single and double exponential decay models in OriginPro 9, and the most reliable value was chosen according to the adjusted $\mathrm{R}^{2}$ value and the shape of the residuals. A relative error of $10 \%$ is typically found among a series of measurements on the same sample. 
Hydration numbers $q$ were obtained by measuring the lifetimes of the same quantity of complex in a PIPES buffered solution in $\mathrm{H}_{2} \mathrm{O}$ and in $\mathrm{D}_{2} \mathrm{O}$ and fitting the difference according to the model of Horrocks et al.,,$^{5-6}$ and Beeby et al. ${ }^{7}$

The fluorescence lifetime decays in the nanosecond range were measured on Fluorolog 3 TCSPC system from Horiba Instruments Inc. The system was equipped with pulsed diode light source NanoLED with excitation wavelength at $315 \mathrm{~nm}$. The data were acquired in the $100 \mathrm{~ns}$ measurement range with peak preset at $10^{4}$ counts in 4096 channels. The repetition rate of the excitation source was $1 \mathrm{MHz}$, and the synchronization delay was $40 \mathrm{~s}$. The scatter light profile (prompt signal, blue in the decay figures) was recorded for each experiment individually in the same quartz cuvette using diluted Ludox solution in Milli-Q water at $315 \mathrm{~nm}$ emission wavelength with similar parameters as were used for the measured sample (red in the decay figures). All measurements were done at room temperature with $10 \mu \mathrm{M}$ concentration of the sample by using $320 \mathrm{~nm}$ bandpass filter and $5 \mathrm{~nm}$ slits width of the detector. Ln complexes and L1 ${ }^{\text {MOM }}$ were measured twice from different stock solutions with $390 \mathrm{~nm}$ emission wavelength, and the solvent was PIPES buffered in distilled water $(\mathrm{pH} \sim 6.5)$. The control experiments were performed with Rose Bengal and Erythrosin B as lifetime standards in $\mathrm{MeOH}$ at 577 and $555 \mathrm{~nm}$ emission wavelengths, respectively (Table S20 and Figures S51-53). The obtained data were fitted in the fluorescence decay analysis software DAS6 using single exponential fit model in Equation (2), where $\tau$ is the sample lifetime, $i$ is times that are represented in channels, A and B are the amplitudes (free in all cases) (Table S18-19 and Figures S54-67).

$$
y(i)=A+B * \exp (-i / \tau)
$$

Crystallography. Measurements were performed using graphite-monochromatized Mo $\mathrm{K}_{\alpha}$ radiation at $150 \mathrm{~K}$ using a Bruker D8 APEX-II equipped with a CCD camera. 
The structure was solved by direct methods (SHELXS-2014) and refined by full-matrix least-squares techniques against $F^{2}$ (SHELXL-2018). The non-hydrogen atoms were refined with anisotropic displacement parameters. The $\mathrm{H}$ atoms of the $\mathrm{CH}_{2} / \mathrm{CH}$ groups were refined with common isotropic displacement parameters for the $\mathrm{H}$ atoms of the same group and idealized geometry. The $\mathrm{H}$ atoms of the methyl groups were refined with common isotropic displacement parameters for the $\mathrm{H}$ atoms of the same group and idealized staggered geometry; one methyl group is modelled as a disordered staggered configuration.

Specific for EuL2 ${ }^{\text {мом}}$ : Lattice $\mathrm{H}_{2} \mathrm{O}$ protons were located on the difference map. Those which could not be located on the difference map were omitted.

Specific for GdL3 ${ }^{\text {MOM: }}$ : The non-coordinated chloride counterions are disordered over numerous sites in the lattice, and have been modelled to reflect this to the best of our ability. The lattice $\mathrm{H}_{2} \mathrm{O}$ protons could not be found on the difference map and were omitted. The methoxymethyl group of the antenna shows a positional disorder which was modelled with occupancies of 0.5 and 0.5 for the two orientations. Solvent accessible voids were treated using the Olex2 solvent mask accounting for 1348 electrons in a $3712 \AA^{3}$ large void (equivalent to approximately $168 \mathrm{H}_{2} \mathrm{O}$ molecules per unit cell, or 21 per asymmetric unit). The final output of solvent mask is appended to the cif file.

Specific for TbL3 $\mathbf{3 0 M}^{\text {мом }}$ The non-coordinated chloride counterions are disordered over numerous sites in the lattice, and have been modelled to reflect this to the best of our ability. The lattice $\mathrm{H}_{2} \mathrm{O}$ protons could not be found on the difference map and were omitted. The methoxymethyl group of the antenna shows a positional disorder which was modelled with occupancies of 0.5 and 0.5 for the two orientations. Solvent accessible voids were treated using the Olex2 solvent mask accounting for 1222 
electrons in a $3525 \AA^{3}$ large void (equivalent to approximately $152 \mathrm{H}_{2} \mathrm{O}$ molecules per unit cell, or 19 per asymmetric unit). The final output of solvent mask is appended to the cif file.

Specific for EuL4a $\mathbf{a}^{\text {MOM}}$-F : Lattice $\mathrm{H}_{2} \mathrm{O}$ protons were located on the difference map. Those which could not be located on the difference map were omitted. The noncoordinated chloride counterions are disordered over numerous sites in the lattice, and have been modelled to reflect this to the best of our ability.

CCDC 1993033, 1993034, 1993035 and 1993036 contain the supplementary crystallographic data for this paper. The data can be obtained free of charge from The Cambridge Crystallographic Data Centre via www.ccdc.cam.ac.uk/structures. 


\section{Synthesis and chemical characterization}

Synthesis of cyclen-based ligands and lanthanide complexes. The neutral complexes $\mathbf{L n L 1}^{\mathrm{Me}}$, LnL1 ${ }^{\mathrm{MOM}}$ and $\mathbf{L n L 1}{ }^{\mathrm{CF3}}$ have been reported before, ${ }^{3}$ and were re-synthesized following the original procedures. Minor changes in the protocols resulted in improved yields and/or simpler purification. These are noted in the protocols.

The complexes carrying a +1 overall charge $\left(\mathbf{L n L} \mathbf{2}^{\mathbf{M O M}}\right)$ were prepared as shown in Scheme S1. The known building block $\mathbf{1}^{1}$ was alkylated on one of the secondary amines with the Nchloroacetylated antenna $\mathbf{2}^{3}$ in the presence of Hünig's base to yield trialkylated $\mathbf{3}$. The remaining secondary amine was then alkylated with bromoacetamide $\mathbf{4 a}$ to give $\mathbf{5}$, which could be deprotected to $\mathbf{L} 2^{\text {MOM }}$ by treatment with a 1:1 mixture of TFA and $\mathrm{CH}_{2} \mathrm{Cl}_{2}$. Heating a mixture of the ligand and $\mathrm{LnCl}_{3}(\mathrm{Ln}=\mathrm{Eu}, \mathrm{Tb}, \mathrm{Gd})$ in $\mathrm{EtOH}: \mathrm{H}_{2} \mathrm{O}$ afforded the $\mathrm{Ln}$ complexes, which could be purified by column chromatography on neutral aluminium oxide.

The starting point for the +2-charged complexes $\left(\mathbf{L n L} \mathbf{3}^{\mathbf{M O M}}\right)$ was the 1,7-diBoc-protected cyclen 6 (Scheme S2). The monoalkylation of $\mathbf{6}$ was possible by reaction with 0.4 equiv. 2. Increasing the amount of added $\mathbf{2}$ afforded large quantities of the overalkylated side-product, which was observed even under the optimized conditions. The isolation of $\mathbf{7}$ required lengthy chromatographic purification. The subsequent steps were straightforward, and consisted of a second alkylation with ethyl bromoacetate $(\mathbf{8})$, the acidic removal of the Boc groups (9), and double alkylation of the revealed secondary amines with bromoacetamide $\mathbf{4 a}$. The ethyl ester 10 was hydrolyzed under basic conditions to yield $\mathbf{L 2}^{\text {MOM }}$. The complexes were synthesized and isolated as described for $\mathbf{L n L 2} \mathbf{2}^{\mathrm{MOM}}$.

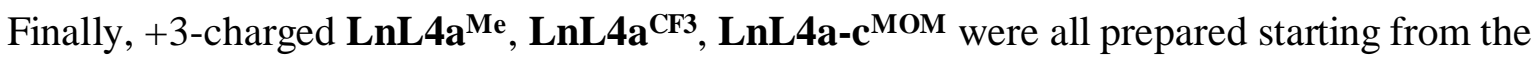
known monoalkylated cyclen derivatives carrying the appropriate antennae (Scheme S3). Trifold alkylation of the secondary amines in $11^{\mathrm{Me}}$ and $\mathbf{1 1}^{\mathrm{CF3}}$ with $4 \mathrm{a}$ and in $\mathbf{1 1}^{\mathrm{MOM}}$ with bromoacetamides $\mathbf{4 a}, \mathbf{4 b}$ or $\mathbf{4 c}$ yielded the ligands in one step. Ligands $\mathbf{L} \mathbf{4 a}^{\mathrm{Me}}, \mathbf{L} \mathbf{4 a} \mathbf{a}^{\mathbf{C F} 3}$ and 
$\mathbf{L 4} \mathbf{a}^{\text {MOM }}$ required only filtration of the product from the reaction mixture after precipitation, whereas more soluble $\mathbf{L} 4 b^{\text {MOM }}$ and L4c $\mathbf{c}^{\text {MOM }}$ were isolated after column chromatography on neutral alumina. Complexation with $\mathrm{LnCl}_{3}$ could be carried out as for the other ligands, and the complexes were isolated in a similar fashion as white solids.

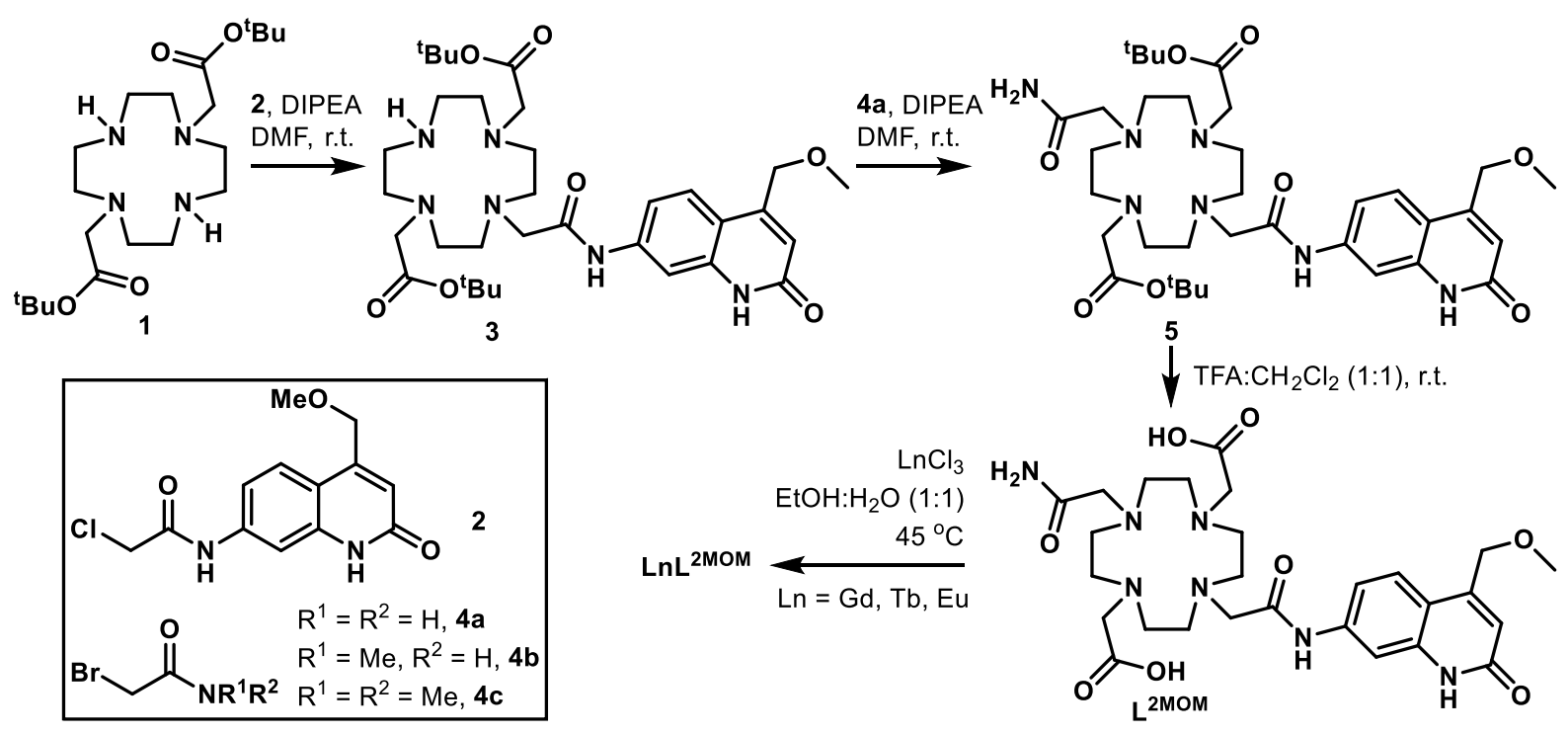

Scheme S1. Synthesis of LnL2 $2^{\text {MOM. }}$

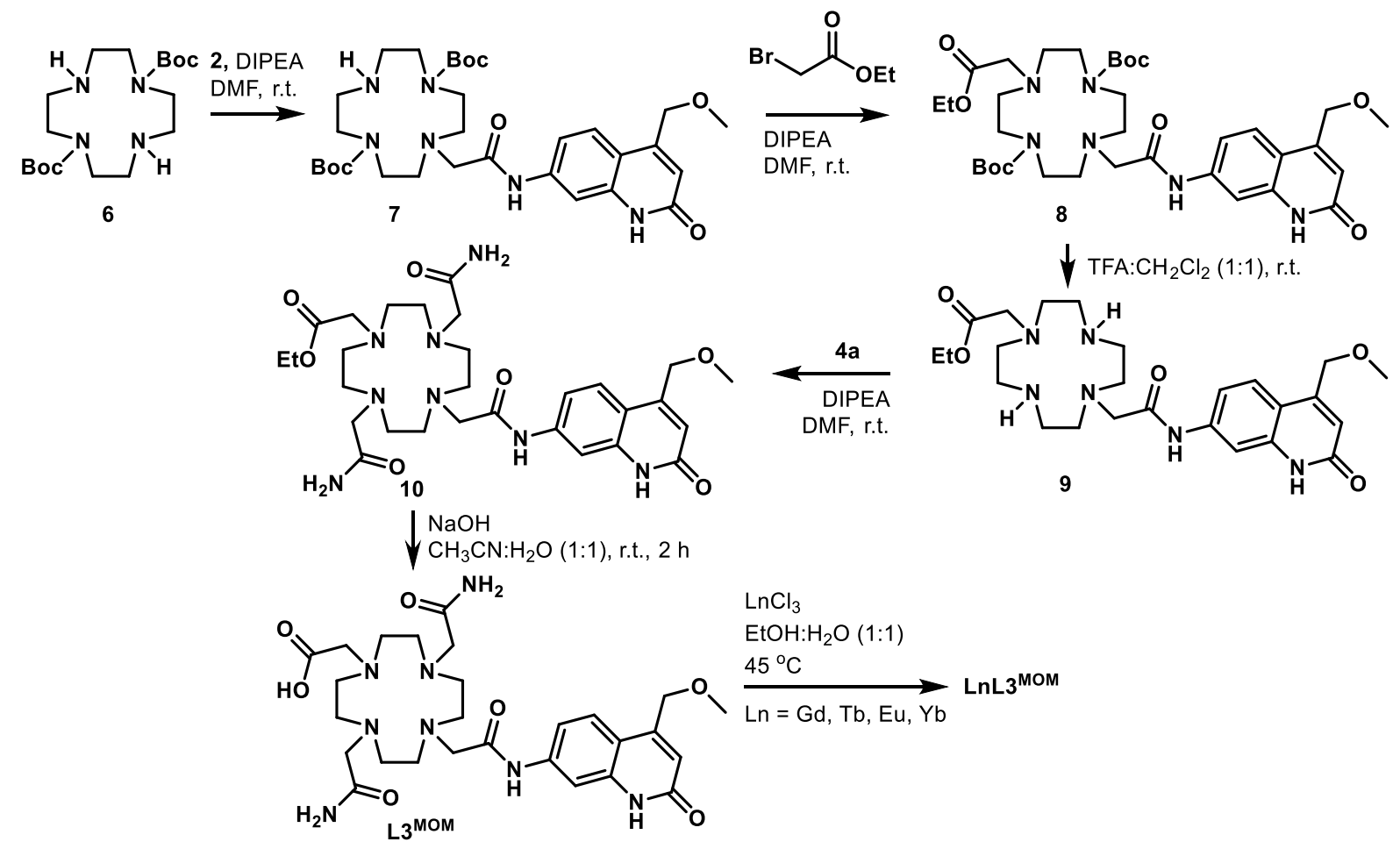

Scheme S2. Synthesis of $\mathbf{L n L 3} 3^{\text {MOM }}$. 


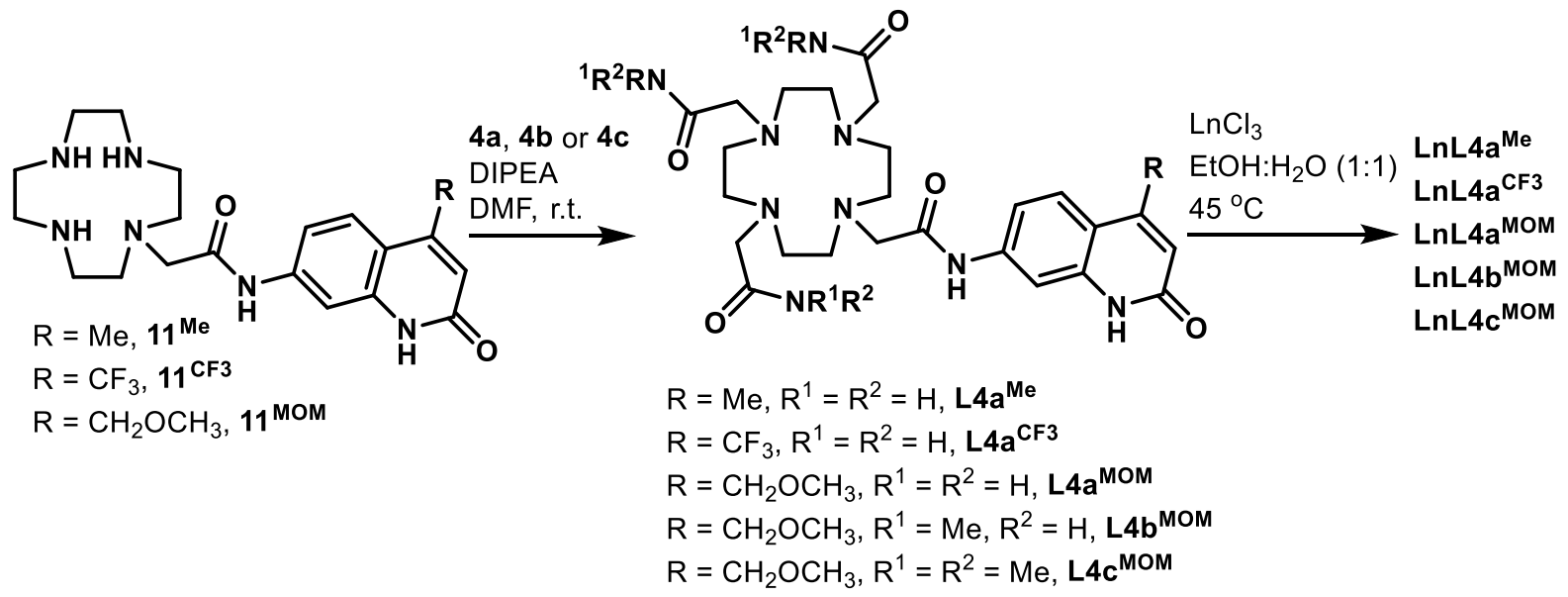

Scheme S3. Synthesis of $\mathbf{L n L 4 a ^ { M e }}, \mathbf{L n L 4 a}^{\mathrm{CF}}, \mathbf{L n L 4 a - c}^{\mathrm{MOM}}$.

\section{Synthetic procedures and characterization data of new compounds}<smiles>COCc1cc(=O)[nH]c2cc(N)ccc12</smiles>

7-amino-4-(methoxymethyl)quinolin-2(1H)-one. Known compound, ${ }^{1}$ prepared in improved yield. A sample of 1,3-diaminobenzene (4.0 g, $37 \mathrm{mmol})$ and methyl-4methoxyacetoacetate $(5.74 \mathrm{~mL}, 44.4 \mathrm{mmol})$ was placed in $25 \mathrm{~mL}$ round bottom flask equipped with a condenser. The mixture was heated at $130{ }^{\circ} \mathrm{C}$ overnight with stirring. The reaction mixture was let cooling down to room temperature after the formation of the bright blue fluorescent product was confirmed by TLC analysis. The thick reaction mixture was diluted with $\mathrm{CH}_{2} \mathrm{Cl}_{2}: \mathrm{MeOH}$ (1:1) mixture and upon sonication a brownish orange solid was precipitated. The latter was filtered and washed with $\mathrm{CH}_{2} \mathrm{Cl}_{2}: \mathrm{MeOH}$ equal mixture to yield the expected product (7.0 g, 92\%). ${ }^{1} \mathrm{H}$ NMR (400 MHz, DMSO-d $) \delta$ ppm 3.35 (s, 3H), 4.52 (s, 2H), $5.77(\mathrm{~s}, 2 \mathrm{H}), 6.07(\mathrm{~s}, 2 \mathrm{H}), 6.38(\mathrm{~d}, J=2.1 \mathrm{~Hz}, 1 \mathrm{H}), 6.43\left(\mathrm{dd}, J_{1}=8.7 \mathrm{~Hz}, J_{2}=2.1 \mathrm{~Hz}, 1 \mathrm{H}\right)$, $7.30(\mathrm{~d}, J=8.7 \mathrm{~Hz}, 1 \mathrm{H}), 11.25(\mathrm{~s}, 1 \mathrm{H}) ;{ }^{13} \mathrm{C}$ NMR $\left(101 \mathrm{MHz}, \mathrm{DMSO}-d_{6}\right) \delta \mathrm{ppm} 58.0,70.5,96.8$ $108.1,110.5,112.7,125.0,141.1,147.3,151.1,162.4 . R P-H P L C ~ t_{R}=4.27 \min (10 \mathrm{~min}$ method 
$\left.10 \% \rightarrow 90 \% \mathrm{CH}_{3} \mathrm{CN}\right)$; ESI-MS obsd 205.16, calcd $205.10(\mathrm{M}+\mathrm{H})^{+}$; HR-ESI-MS obsd 227.0797, calcd 227.0791 [(M $\left.+\mathrm{Na})^{+}, \mathrm{M}=\mathrm{C}_{11} \mathrm{H}_{12} \mathrm{~N}_{2} \mathrm{O}_{2}\right]$.

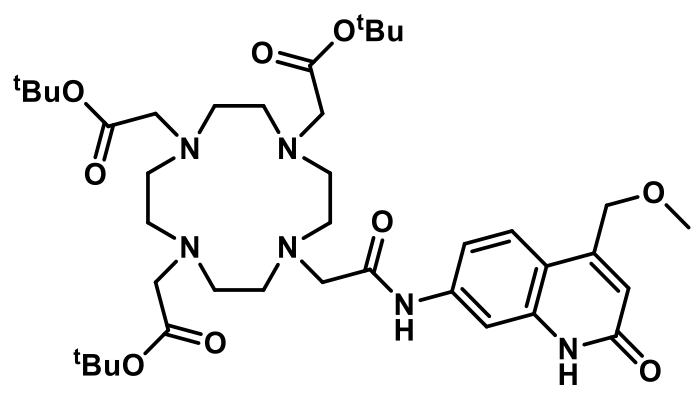

Protected L1 ${ }^{\text {MOM }}$. Known compound, ${ }^{2}$ new procedure. A sample of $\mathbf{1 1}^{\text {MOM }}$ (50 mg, 0.12 mmol) was dissolved in DMF (1.67 mL) followed by the addition of DIPEA (104.5 $\mu \mathrm{L}, 0.60$ mmol). The reaction mixture was stirred at room temperature overnight after the addition of tert-butyl bromoacetate $(57 \mu \mathrm{L}, 0.384 \mathrm{mmol})$. The next day LC-MS analysis showed full conversion of the monoalkylated cyclen to the target product. The reaction mass was directly loaded on a neutral alumina column which had been conditioned with $100 \% \mathrm{CH}_{3} \mathrm{CN}$. Elution with $\mathrm{CH}_{3} \mathrm{CN}: \mathrm{H}_{2} \mathrm{O} \quad(98: 2 \rightarrow 85: 15)$ removed DMF and DIPEA. A second column chromatography was performed on silica gel with $\mathrm{CHCl}_{3}$ :Acetone:MeOH mixture (90:5:5 $\rightarrow$ 60:20:20) to remove residues of solvent and base. Final purification step yielded off-white solid (47 mg, 52\%). ${ }^{1} \mathrm{H}$ NMR (400 MHz, DMSO- $\left.d_{6}\right) \delta \mathrm{ppm} 1.15-1.64(\mathrm{~m}, 27 \mathrm{H},+2 \mathrm{H}$ DIPEA $\cdot \mathrm{HBr})$, 1.67-3.80 (m, 27H, + 8H DMSO, $\mathrm{H}_{2} \mathrm{O}$, DIPEA·HBr), $4.62(\mathrm{~s}, 2 \mathrm{H}), 6.37(\mathrm{~s}, 1 \mathrm{H}), 7.15(\mathrm{~d}, J=$ $1.3 \mathrm{~Hz}, 1 \mathrm{H}), 7.47(\mathrm{~d}, J=8.9 \mathrm{~Hz}, 1 \mathrm{H}), 7.82(\mathrm{~d}, J=8.9 \mathrm{~Hz}, 1 \mathrm{H}), 10.69(\mathrm{~s}, 1 \mathrm{H}), 11.75$ (br, 1H); ${ }^{13} \mathrm{C}$ NMR (101 MHz, DMSO- $\left.d_{6}\right) \delta$ ppm 27.6, 47.2-48.7, 51.5-52.9, 55.3, 56.5, 58.0, 70.2, 81.1, $81.2,105.0,113.6,114.5,117.5,124.3,139.5,140.6,146.9,161.9,171.2,172.5 ;$ RP-HPLC $t_{R}$ $=7.30 \min \left(0-12 \min : 10 \% \rightarrow 50 \% \mathrm{CH}_{3} \mathrm{CN} \& 12-14 \min : 50 \% \rightarrow 90 \%\right)$; ESI-MS obsd 761.42 , calcd $761.48(\mathrm{M}+3 \mathrm{H})^{3+}$. HR-ESI-MS obsd 781.4488, calcd $781.4470\left[(\mathrm{M}+\mathrm{Na})^{+}, \mathrm{M}=\right.$ $\left.\mathrm{C}_{39} \mathrm{H}_{62} \mathrm{~N}_{6} \mathrm{O}_{9}\right]$. 
<smiles>COCc1cc(=O)[nH]c2cc(NC(=O)CCl)ccc12</smiles>

2. Known compound, ${ }^{2}$ isolated sample of analytical purity. 7-amino-4(methoxymethyl)quinolin-2(1H)-one $(4.5 \mathrm{~g}, 22.0 \mathrm{mmol})$ and $\mathrm{Et}_{3} \mathrm{~N}(9.21 \mathrm{~mL}, 66.1 \mathrm{mmol})$ were dissolved in DMF $(67 \mathrm{~mL})$, and the solution was cooled at $0{ }^{\circ} \mathrm{C}$ in ice-water bath. Chloroacetyl chloride $(2.1 \mathrm{~mL}, 26 \mathrm{mmol})$ was added to the reaction mixture and it was stirred at $0{ }^{\circ} \mathrm{C}$ for 30 min. Then the cooling bath was removed and the reaction mass was stirred for $1 \mathrm{~h}$. The excess of chloroacetyl chloride was added $(1.05 \mathrm{~mL}, 13.2 \mathrm{mmol})$ under the same conditions for the full conversion of 7-amino-4-(methoxymethyl)quinolin- $2(1 \mathrm{H})$-one, and the reaction mixture was stirred at room temperature for another hour. The formed reaction mass was poured into a separation funnel containing $\mathrm{CH}_{2} \mathrm{Cl}_{2}(340 \mathrm{~mL}), \mathrm{MeOH}(85 \mathrm{~mL})$ and $\mathrm{H}_{2} \mathrm{O}(425 \mathrm{~mL})$. The aqueous phase was extracted with $2 \times 425 \mathrm{~mL} \mathrm{CH}_{2} \mathrm{Cl}_{2}$. The combined organic phases were dried over $\mathrm{MgSO}_{4}$, filtered, and the filtrate was concentrated under reduced pressure. DMF residues were removed by co-evaporation with toluene. The viscous brown residue was recrystallized from $\mathrm{CH}_{2} \mathrm{Cl}_{2}$ :MeOH (1:1) mixture. The purple-grey precipitate was collected, and the solid was washed with a small amount of $\mathrm{CH}_{2} \mathrm{Cl}_{2}$ :MeOH equal mixture. The mass of the solid was $2.9 \mathrm{~g} \mathrm{(46 \% ).} \mathrm{The} \mathrm{filtrate} \mathrm{was} \mathrm{concentrated,} \mathrm{and} \mathrm{the} \mathrm{crude} \mathrm{product} \mathrm{was} \mathrm{purified} \mathrm{on}$ a silica gel column by elution with $\mathrm{CH}_{2} \mathrm{Cl}_{2}: \mathrm{Et}_{2} \mathrm{O}$ :Isopropanol $(70: 30: 0 \rightarrow 70: 26: 4 \rightarrow 70: 22: 8 \rightarrow 70: 20: 10)$, yielding a brown analytically pure solid (237 mg, 4\%): ${ }^{1} \mathrm{H}$ NMR (400 MHz, DMSO-d $) \delta$ ppm 3.38 (s, 3H), $4.28(\mathrm{~s}, 2 \mathrm{H}), 4.62(\mathrm{~s}, 2 \mathrm{H}), 6.39$ (s, 1H), $7.31(\mathrm{~d}, J=9.0 \mathrm{~Hz}, 1 \mathrm{H}), 7.61(\mathrm{~d}, J=9.0 \mathrm{~Hz}, 1 \mathrm{H}), 7.78(\mathrm{~s}, 1 \mathrm{H}), 10.58(\mathrm{~s}, 1 \mathrm{H}), 11.72(\mathrm{~s}$, $1 \mathrm{H}) ;{ }^{13} \mathrm{C}$ NMR (101 MHz, DMSO- $\left.d_{6}\right) \delta \mathrm{ppm} 43.7,58.1,70.2,104.9,113.7,113.8,117.6,125.0$, $139.7,140.2,147.0,162.0,165.1 ; \mathrm{RP}-\mathrm{HPLC} \mathrm{t}_{\mathrm{R}}=4.97 \mathrm{~min}(10 \mathrm{~min} \operatorname{method} 10 \% \rightarrow 90 \%$ 
$\left.\mathrm{CH}_{3} \mathrm{CN}\right)$; ESI-MS obsd 283.79, calcd $283.73(\mathrm{M}+3 \mathrm{H})^{3+}$. HR-ESI-MS obsd 303.0503, calcd $303.0507\left[(\mathrm{M}+\mathrm{Na})^{+}, \mathrm{M}=\mathrm{C}_{13} \mathrm{H}_{13} \mathrm{~N}_{2} \mathrm{O}_{3} \mathrm{Cl}\right]$.

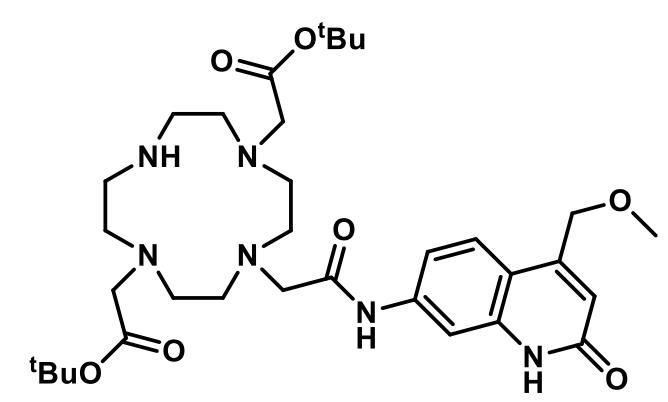

3. A sample of 1 (1.0 g, $2.5 \mathrm{mmol})$ was dissolved in DMF (33 $\mathrm{mL})$ and DIPEA $(870 \mu \mathrm{L}$, $5.00 \mathrm{mmol})$ was added, followed by the addition of $2(350 \mathrm{mg}, 1.25 \mathrm{mmol})$. The reaction mixture was stirred overnight at room temperature. HPLC-MS analysis showed full conversion of the limiting reagent, and alongside the expected product small amounts of dialkylated product was observed $(<10 \%)$. Sequential column chromatography on alumina columns $\left(1^{\text {st }}\right.$, $2^{\text {nd }}: \mathrm{CH}_{3} \mathrm{CN}: \mathrm{H}_{2} \mathrm{O} 97: 3 \rightarrow 9: 1 ; 3^{\text {rd }}, 4^{\text {th }}: \mathrm{CHCl}_{3}:$ Acetone:MeOH 100:0:0 $\rightarrow$ 90:5:5) yielded the pure product as an off-white solid (662 mg, 82\%). ${ }^{1} \mathrm{H}$ NMR (400 MHz, DMSO- $\left.d_{6}\right) \delta$ ppm 1.34 (s, 18H), 2.53-3.30 (m, 22H, + 1.9H solvents), $3.39(\mathrm{~s}, 3 \mathrm{H}), 4.64(\mathrm{~s}, 2 \mathrm{H}), 6.38(\mathrm{~s}, 1 \mathrm{H}), 7.16$ (br, 1H), $7.66(\mathrm{~d}, J=8.7 \mathrm{~Hz}, 1 \mathrm{H}), 7.85(\mathrm{br}, 1 \mathrm{H}), 10.24(\mathrm{br}, 1 \mathrm{H}), 11.75(\mathrm{br}, 1 \mathrm{H}) ;{ }^{13} \mathrm{C}$ NMR $(101$ MHz, DMSO- $\left.d_{6}\right) \delta$ ppm 27.8, 47.2-47.7, 50.8-52.0, 55.1, 56.3, 58.2, 70.3, 80.3, 80.4, 103.7, $112.8,113.4,117.3,125.2,140.0,140.1,147.0,162.0,170.3,170.8 ; \mathrm{RP}-H P L C t_{R}=5.55 \mathrm{~min}$ (16 min method, 0-12 min: $10 \% \rightarrow 50 \% \mathrm{CH}_{3} \mathrm{CN} \& 12-14 \mathrm{~min}: 50 \% \rightarrow 90 \%$ ); ESI-MS obsd 645.36, calcd 645.40 $(\mathrm{M}+\mathrm{H})^{+}$; HR-ESI-MS obsd 645.3978, calcd $645.3970\left[(\mathrm{M}+\mathrm{H})^{+}, \mathrm{M}=\right.$ $\mathrm{C}_{33} \mathrm{H}_{52} \mathrm{~N}_{6} \mathrm{O}_{7}$ ]. 


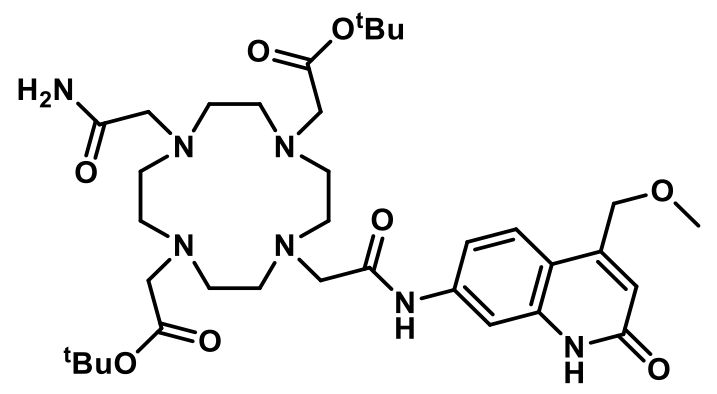

5. A sample of 3 (300 mg, $0.465 \mathrm{mmol})$ was dissolved in DMF (6.2 mL). DIPEA (243 $\mu \mathrm{L}$, $1.40 \mathrm{mmol}$ ) was added to the solution, followed by 2 -bromoacetamide (4a, $87 \mathrm{mg}, 0.63 \mathrm{mmol})$. The reaction mixture was stirred overnight at room temperature. The reaction mixture was directly loaded onto a neutral alumina column which had been conditioned with $100 \%$ acetonitrile after LC-MS analysis showed full conversion to the product. Column chromatography $\left(\mathrm{CH}_{3} \mathrm{CN}: \mathrm{H}_{2} \mathrm{O}\right)$ using a low gradient yielded the product as an off-white solid (325 mg, quant.). ${ }^{1} \mathrm{H}$ NMR (400 MHz, DMSO- $\left.d_{6}\right) \delta \mathrm{ppm} 1.37(\mathrm{~s}, 18 \mathrm{H}), 1.87-3.66(\mathrm{~m}, 27 \mathrm{H},+$ 7.2H DMSO, $\mathrm{H}_{2} \mathrm{O}, \mathrm{DMF}$, DIPEA·HBr), 4.61 (s, 2H), 6.37 (s, 1H), 7.15 (s, 1H, NH), 7.33 (d, J $=1.4 \mathrm{~Hz}, 1 \mathrm{H}), 7.51(\mathrm{~d}, J=8.9 \mathrm{~Hz}, 1 \mathrm{H}), 7.68(\mathrm{~s}, 1 \mathrm{H}, \mathrm{NH}), 7.70\left(\mathrm{dd}, J_{l}=9.0 \mathrm{~Hz}, J_{2}=1.5 \mathrm{~Hz}\right.$, 1H), 10.69 (s, 1H), 11.73 (s, 1H); ${ }^{13} \mathrm{C}$ NMR (101 MHz, DMSO- $\left.d_{6}\right) \delta$ ppm 27.7, 49.0-52.4, 55.6, $55.8,57.0,58.1,70.2,81.0,105.0,113.6,114.4,117.5,124.4,139.6,140.6,146.8,161.9,171.3$, $171.8,173.5 ; \mathrm{RP}-\mathrm{HPLC} \mathrm{t}_{\mathrm{R}}=1.73,5.03 \mathrm{~min}\left(16 \mathrm{~min}\right.$ method, $0-12 \mathrm{~min}: 10 \% \rightarrow 50 \% \mathrm{CH}_{3} \mathrm{CN} \&$ 12-14 min: 50\% $\rightarrow 90 \%)$; ESI-MS obsd 702.80, calcd $702.42(\mathrm{M}+\mathrm{H})^{+}$; HR-ESI-MS obsd 702.4188, calcd 702.4185 $\left[(\mathrm{M}+\mathrm{H})^{+}, \mathrm{M}=\mathrm{C}_{35} \mathrm{H}_{55} \mathrm{~N}_{7} \mathrm{O}_{8}\right]$.

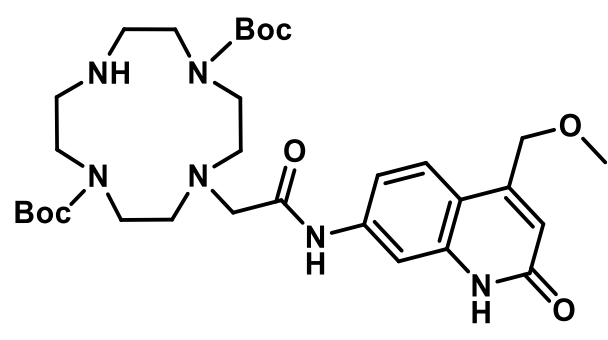

7. A sample of 6 (3.074 g, $8.25 \mathrm{mmol})$ was dissolved in DMF (100 mL), and DIPEA (2.16 $\mathrm{mL}, 12.4 \mathrm{mmol})$ was added. 2 (927 mg, $3.30 \mathrm{mmol})$ dissolved in DMF (10 mL) was added 
dropwise to the solution. The reaction mixture was stirred overnight at room temperature. The reaction mixture was directly loaded onto neutral alumina column which had been conditioned with acetonitrile after the full consumption of the limiting reagent. Elution with $\mathrm{CH}_{3} \mathrm{CN}: \mathrm{H}_{2} \mathrm{O}$ $(100: 0 \rightarrow 70: 30)$ removed most of the DMF and the base. A second chromatographic step on silica gel using $\mathrm{CH}_{2} \mathrm{Cl}_{2}: \mathrm{MeOH}(80: 20 \rightarrow 55: 45)$ removed the solvent and base residues, and yielded a yellow-white solid (1.62 g, 80\%). ${ }^{1} \mathrm{H}$ NMR (400 MHz, DMSO- $\left.d_{6}\right) \delta$ ppm $1.12-1.55$ (s, 18H), 2.53-3.76 (m, 22H, + 0.4H H $\left.{ }_{2} \mathrm{O}\right), 4.62(\mathrm{~s}, 2 \mathrm{H}), 6.36(\mathrm{~s}, 1 \mathrm{H}), 7.34(\mathrm{~d}, J=8.6 \mathrm{~Hz}, 1 \mathrm{H})$, $7.58(\mathrm{~d}, J=8.8 \mathrm{~Hz}, 1 \mathrm{H}), 7.85(\mathrm{~s}, 1 \mathrm{H}), 10.36(\mathrm{br}, 1 \mathrm{H}), 11.65(\mathrm{~s}, 1 \mathrm{H}) ;{ }^{13} \mathrm{C}$ NMR $(101 \mathrm{MHz}$, DMSO- $\left.d_{6}\right) \delta$ ppm $28.0,46.5,46.6,47.2,48.8,48.9,49.2,49.3,49.8,52.5,53.0,53.2,53.8$, $55.1,58.1,70.2,78.6,104.8,113.3,113.7,117.2,124.6,139.7,140.7,146.9,154.9,155.5$, $162.0,170.1 ; \mathrm{RP}-\mathrm{HPLC} \mathrm{t}_{\mathrm{R}}=7.98 \mathrm{~min}\left(16 \mathrm{~min}\right.$ method, $0-12 \mathrm{~min}: 10 \% \rightarrow 50 \% \mathrm{CH}_{3} \mathrm{CN} \& 12-$ $14 \min : 50 \% \rightarrow 90 \%)$; ESI-MS obsd 617.40, calcd $617.37(\mathrm{M}+\mathrm{H})^{+}$; HR-ESI-MS obsd 617.3681, calcd 617.3657 $\left[(\mathrm{M}+\mathrm{H})^{+}, \mathrm{M}=\mathrm{C}_{31} \mathrm{H}_{48} \mathrm{~N}_{6} \mathrm{O}_{7}\right]$.

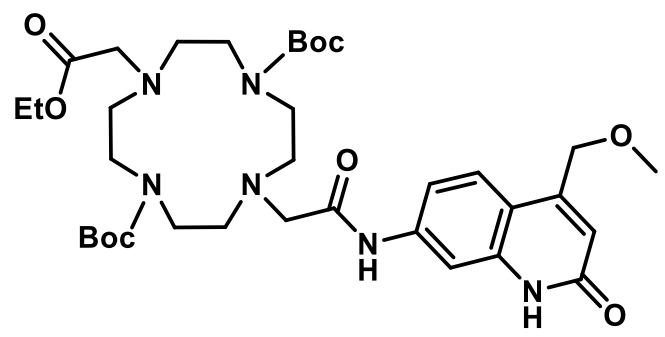

8. A sample of 7 (1.16 g, $1.88 \mathrm{mmol})$ was dissolved in DMF (25 mL) and DIPEA (981 $\mu \mathrm{L}$, $5.63 \mathrm{mmol})$ was added. Ethyl bromoacetate $(250 \mu \mathrm{L}, 2.25 \mathrm{mmol})$ was added to the reaction mixture with a pipette and the formed solution was let to stir for overnight at room temperature. The reaction mixture was directly loaded onto neutral alumina column which had been conditioned with acetonitrile when LC-MS analysis showed full conversion to the product. Elution with $\mathrm{CH}_{3} \mathrm{CN}: \mathrm{H}_{2} \mathrm{O}(100: 0 \rightarrow 90: 10)$ removed most of the DMF and the base. A second chromatographic step on silica gel using $\mathrm{CH}_{2} \mathrm{Cl}_{2}: \mathrm{EtOH}(100: 0 \rightarrow 90: 10 \rightarrow 80: 20)$ removed the solvent and base residues, and yielded a white solid (1.14 g, 86\%). ${ }^{1} \mathrm{H}$ NMR (400 MHz, DMSO- 
$\left.d_{6}\right) \delta \mathrm{ppm} 1.10-1.53(\mathrm{~m}, 21 \mathrm{H}), 2.58-3.02(\mathrm{~m}, 8 \mathrm{H}), 3.04-3.74\left(\mathrm{~m}, 15 \mathrm{H},+0.9 \mathrm{H} \mathrm{H}_{2} \mathrm{O}\right), 4.09$ (q, $J$ $=7.1 \mathrm{~Hz}, 2 \mathrm{H}), 4.63(\mathrm{~s}, 2 \mathrm{H}), 6.37(\mathrm{~s}, 1 \mathrm{H}), 7.32-7.36(\mathrm{~m}, 1 \mathrm{H}), 7.57(\mathrm{~d}, J=8.8 \mathrm{~Hz}, 1 \mathrm{H}), 7.82-$ $8.04(\mathrm{~m}, 1 \mathrm{H}), 9.59,9.97,10.15(3 \times \mathrm{br}, 1 \mathrm{H}), 11.65(\mathrm{~s}, 1 \mathrm{H}) ;{ }^{13} \mathrm{C} \mathrm{NMR}\left(101 \mathrm{MHz}, \mathrm{DMSO}-d_{6}\right) \delta$ ppm 14.2, 28.0, 44.7, 46.2, 46.5, 47.1, 47.9, 51.2, 52.2, 53.2, 53.5, 54.1, 54.6, 58.0, 59.8, 70.2, $78.5,78.8,104.9,113.3,113.7,117.2,124.4,139.7,140.2,146.9,154.9,155.2,155.6,161.9$, $170.4,170.6,170.9 ; \mathrm{RP}-H P L C t_{\mathrm{R}}=9.85 \mathrm{~min}\left(16 \mathrm{~min}\right.$ method, $0-12 \mathrm{~min}: 10 \% \rightarrow 50 \% \mathrm{CH}_{3} \mathrm{CN}$ \& 12-14 min: 50\% $\rightarrow 90 \%)$; ESI-MS obsd 705.48, calcd $705.42(\mathrm{M}+3 \mathrm{H})^{3+} ;$ HR-ESI-MS obsd 703.4048, calcd $703.4025\left[(\mathrm{M}+\mathrm{H})^{+}, \mathrm{M}=\mathrm{C}_{35} \mathrm{H}_{54} \mathrm{~N}_{6} \mathrm{O}_{9}\right]$.

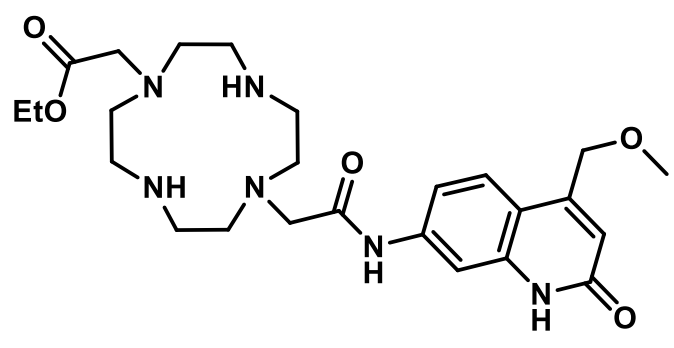

9. A sample of $\mathbf{8}(150 \mathrm{mg}, 0.213 \mathrm{mmol})$ was dissolved in $\mathrm{CH}_{2} \mathrm{Cl}_{2}(2.4 \mathrm{~mL})$, and TFA (2.4 $\mathrm{mL}$ ) was added dropwise. The solution was stirred at room temperature. TLC analysis showed complete cleavage of the Boc protective groups after $2 \mathrm{~h}$. The reaction mixture was concentrated under reduced pressure to receive an oily residue which was dissolved in $\mathrm{CH}_{2} \mathrm{Cl}_{2}: \mathrm{MeOH}: \mathrm{NH}_{4} \mathrm{OH}$ (4 mL, 50:47:3). The mixture was loaded onto a silica column. Column chromatography $\left(\mathrm{CH}_{2} \mathrm{Cl}_{2}: \mathrm{MeOH}: \mathrm{NH}_{4} \mathrm{OH}, 50: 47: 3\right)$ yielded a white solid $(74.4 \mathrm{mg}, 69 \%) ;{ }^{1} \mathrm{H}$ NMR (400 MHz, DMSO- $\left.d_{6}\right) \delta$ ppm $1.13(\mathrm{t}, J=7.1 \mathrm{~Hz}, 3 \mathrm{H}), 1.84-3.74$ (m, 25H, + 6.1H DMSO; $\left.\mathrm{H}_{2} \mathrm{O}\right), 3.98(\mathrm{q}, J=7.1 \mathrm{~Hz}, 2 \mathrm{H}), 4.61(\mathrm{~s}, 2 \mathrm{H}), 6.36(\mathrm{~s}, 1 \mathrm{H}), 7.28(\mathrm{~d}, J=8.5 \mathrm{~Hz}, 1 \mathrm{H}), 7.57(\mathrm{~d}, J=$ $8.4 \mathrm{~Hz}, 1 \mathrm{H}), 7.83(\mathrm{~s}, 1 \mathrm{H}), 10.32(\mathrm{br}, 1 \mathrm{H}), 11.72(\mathrm{~s}, 1 \mathrm{H}) ;{ }^{13} \mathrm{C} \mathrm{NMR}\left(101 \mathrm{MHz}, \mathrm{DMSO}-d_{6}\right) \delta \mathrm{ppm}$ $14.1,45.3,51.8,52.5,55.8,58.1,59.8,70.3,104.7,113.3,114.0,117.2,124.5,139.7,140.5$, 146.9, 162.0, 170.3, 171.4; RP-HPLC $\mathrm{t}_{\mathrm{R}}=1.22 \min (16 \mathrm{~min}$ method, $0-12 \mathrm{~min}: 10 \% \rightarrow 50 \%$ $\mathrm{CH}_{3} \mathrm{CN} \& 12-14$ min: $\left.50 \% \rightarrow 90 \%\right)$; ESI-MS obsd 505.27, calcd $505.31(\mathrm{M}+2 \mathrm{H})^{2+}$; HR-ESIMS obsd 503.2986, calcd 503.2976 [(M+ H $\left.)^{+}, \mathrm{M}=\mathrm{C}_{25} \mathrm{H}_{38} \mathrm{~N}_{6} \mathrm{O}_{5}\right]$. 


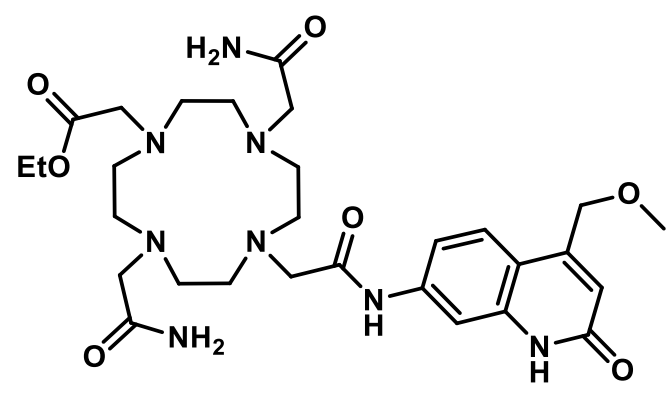

10. A sample of 7 (350 mg, $0.696 \mathrm{mmol})$ was dissolved in DMF (9.3 mL) upon sonication. DIPEA (485 $\mu \mathrm{L}, 2.79$ mmol) was added. A few minutes later 2-bromoacetamide (4a, $211 \mathrm{mg}$, $1.53 \mathrm{mmol}$ ) was added. Stirring was continued at room temperature overnight. LC-MS analysis of the pale-yellow transparent solution indicated the full conversion of the starting materials. One day later the expected product precipitated out as a white solid. The solid was filtered, and the filter cake was washed with a small volume of $\mathrm{CH}_{3} \mathrm{CN}: \mathrm{MeOH}$ (1:1), yielding the product (225 mg, 52\%). The filtrate was concentrated under reduced pressure, and the residue was purified by column chromatography. Gradient elution with $\mathrm{CH}_{3} \mathrm{CN}: \mathrm{H}_{2} \mathrm{O}$ (100:0 $\left.\rightarrow 70: 30\right)$ yielded a white solid (177 mg, 41\%). ${ }^{1} \mathrm{H}$ NMR (400 MHz, DMSO- $\left.d_{6}\right) \delta$ ppm $1.18(\mathrm{t}, J=7.1$ $\mathrm{Hz}, 3 \mathrm{H}), 2.55-3.12(\mathrm{~m}, 20 \mathrm{H},+1.4 \mathrm{H}$ solvents $), 3.14-3.48\left(\mathrm{~m}, 7 \mathrm{H},+2.9 \mathrm{H} \mathrm{H}_{2} \mathrm{O}\right), 4.07(\mathrm{q}, J=7.1$ $\mathrm{Hz}, 2 \mathrm{H}), 4.62(\mathrm{~s}, 2 \mathrm{H}), 6.36(\mathrm{~s}, 1 \mathrm{H}), 6.89(\mathrm{~d}, J=1.9 \mathrm{~Hz}, 2 \mathrm{H}, \mathrm{NH}), 7.33\left(\mathrm{dd}, J_{l}=8.8 \mathrm{~Hz}, J_{2}=1.6\right.$ $\mathrm{Hz}, 1 \mathrm{H}), 7.58(\mathrm{~d}, J=8.8 \mathrm{~Hz}, 1 \mathrm{H}), 7.63(\mathrm{~s}, J=1.9 \mathrm{~Hz}, 2 \mathrm{H}, \mathrm{NH}), 7.82(\mathrm{~d}, J=1.3 \mathrm{~Hz}, 1 \mathrm{H}), 10.17$ (s, $1 \mathrm{H}), 11.62(\mathrm{~s}, 1 \mathrm{H}) ;{ }^{13} \mathrm{C}$ NMR (101 MHz, DMSO- $\left.d_{6}\right) \delta \mathrm{ppm} 14.2$, 51.9, 52.9, 53.0, 53.3, 55.0, $57.8,58.1,59.7,70.3,104.7,113.4,113.9,117.2,124.7,139.7,140.4,147.0,162.0,170.1$, 170.8, 173.0. RP-HPLC $t_{\mathrm{R}}=1.33 \mathrm{~min}\left(16 \mathrm{~min}\right.$ method, $0-12 \mathrm{~min}: 10 \% \rightarrow 50 \% \mathrm{CH}_{3} \mathrm{CN} \& 12-$ 14 min: $50 \% \rightarrow 90 \%)$; ESI-MS obsd 617.36, calcd $617.34(\mathrm{M}+\mathrm{H})^{+}$; HR-ESI-MS obsd 639.3238, calcd 639.3225 [(M + Na) $\left.{ }^{+}, \mathrm{M}=\mathrm{C}_{29} \mathrm{H}_{44} \mathrm{~N}_{8} \mathrm{O}_{7}\right]$.

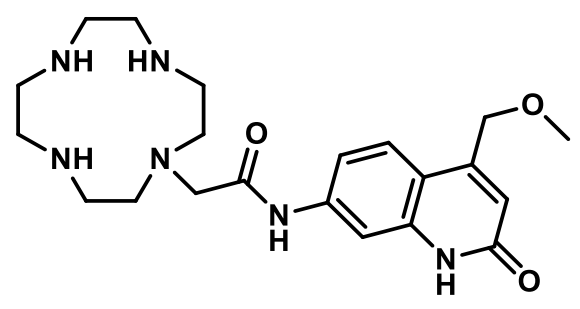


11 ${ }^{\text {MOM }}$. Known compound, ${ }^{2}$ prepared in improved yield. Cyclen $(1032 \mathrm{mg}, 5.990 \mathrm{mmol})$ was dissolved in $\mathrm{CHCl}_{3}(12 \mathrm{~mL})$. The chloroacetylated MOM carbostyril 2 (560 mg, 1.995 mmol) was suspended in the mixture of $\mathrm{CHCl}_{3}(7 \mathrm{~mL})$ and DMF $(4.3 \mathrm{~mL})$. The resulting light brown suspension was stirred overnight at room temperature. On the following day the color of the precipitate had changed to white-grey. The reaction mixture was filtered, and the solid was washed twice with a small amount of $\mathrm{CHCl}_{3}: \mathrm{MeOH}$ (4:1) mixture. A light grey solid was obtained in high yield (802 mg, 97\%). ${ }^{1} \mathrm{H}$ NMR (400 MHz, DMSO- $\left.d_{6}\right) \delta$ ppm 2.64-2.86 (m, $16 \mathrm{H}), 3.38(\mathrm{~s}, 3 \mathrm{H}), 3.49(\mathrm{~s}, 2 \mathrm{H}), 4.62(\mathrm{~s}, 2 \mathrm{H}), 6.36(\mathrm{~s}, 1 \mathrm{H}), 6.92(\mathrm{br}, 3 \mathrm{H}, \mathrm{NH}), 7.42\left(\mathrm{dd}, J_{l}=8.8\right.$ $\left.\mathrm{Hz}, J_{2}=1.5 \mathrm{~Hz}, 1 \mathrm{H}\right), 7.59\left(\mathrm{~d}, J_{1}=8.8 \mathrm{~Hz}, 1 \mathrm{H}\right), 7.80(\mathrm{~d}, J=1.5 \mathrm{~Hz}, 1 \mathrm{H}), 11.69(\mathrm{br}, 1 \mathrm{H}) ;{ }^{13} \mathrm{C}$ NMR (101 MHz, DMSO- $\left.d_{6}\right) \delta$ ppm 44.7, 45.5, 46.7, 51.4, 58.1, 58.3, 70.2, 104.6, 113.3, 113.8, 117.2, 124.7, 139.7, 140.6, 146.9, 162.0, 170.5. RP-HPLC $t_{R}=1.18 \min (16 \min$ method, $0-12$ min: $10 \% \rightarrow 50 \% \mathrm{CH}_{3} \mathrm{CN} \& 12-14$ min: $\left.50 \% \rightarrow 90 \%\right)$; ESI-MS obsd 417.27, calcd 417.26 (M+ $\mathrm{H})^{+}$; HR-ESI-MS obsd 417.2613, calcd 417.2609 [(M+H)+, $\left.\mathrm{M}=\mathrm{C}_{21} \mathrm{H}_{32} \mathrm{~N}_{6} \mathrm{O}_{3}\right]$.

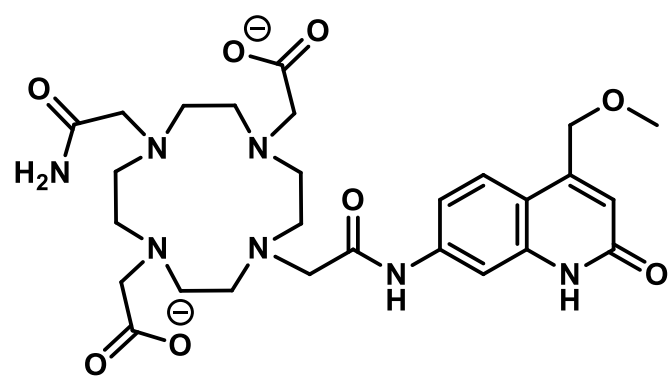

$\mathbf{L 2}^{\mathrm{MOM}}$. A sample of $\mathbf{5}$ was dissolved in $\mathrm{CH}_{2} \mathrm{Cl}_{2}$, and TFA was added dropwise to the solution with a syringe. The mixture was stirred overnight at room temperature. The solvents were removed under reduced pressure after the full conversion to the product. The oily residue was dissolved in $\mathrm{CH}_{3} \mathrm{CN}: \mathrm{H}_{2} \mathrm{O}: \mathrm{NH}_{4} \mathrm{OH}$ (8:1.5:0.5, $\left.10 \mathrm{~mL}\right)$. Column chromatography on neutral alumina $\left(\mathrm{CH}_{3} \mathrm{CN}: \mathrm{H}_{2} \mathrm{O}: \mathrm{NH}_{4} \mathrm{OH}, 90: 10: 0 \rightarrow 80: 15: 5 \rightarrow 70: 25: 5\right)$ yielded an ivory solid $(260 \mathrm{mg}$, quant.). ${ }^{1} \mathrm{H}$ NMR (400 MHz, $\left.\mathrm{D}_{2} \mathrm{O}\right) \delta$ ppm 2.74-4.35 (m, 27H, + 2.35H MeOH), $4.51(\mathrm{~s}, 2 \mathrm{H})$, $6.35(\mathrm{~s}, 1 \mathrm{H}), 6.97(\mathrm{~d}, J=8.7 \mathrm{~Hz}, 1 \mathrm{H}), 7.26(\mathrm{~d}, J=8.9 \mathrm{~Hz}, 1 \mathrm{H}), 7.49(\mathrm{~s}, 1 \mathrm{H}) ;{ }^{13} \mathrm{C}$ NMR $(101$ 
$\left.\mathrm{MHz}, \mathrm{D}_{2} \mathrm{O}\right) \delta \mathrm{ppm} 48.1,48.4,50.9,51.7,55.2,56.1,56.6,58.5,70.2,105.3,114.2,115.2,124.2$, $138.0,139.8,148.7,164.1,169.9,170.8,175.3 ; \mathrm{RP}-\mathrm{HPLC} \mathrm{t}_{\mathrm{R}}=2.47 \mathrm{~min}$ (16 min method, $0-12$ min: $10 \% \rightarrow 50 \% \mathrm{CH}_{3} \mathrm{CN} \& 12-14$ min: $\left.50 \% \rightarrow 90 \%\right)$; ESI-MS obsd 590.69, calcd $590.29(\mathrm{M}+$ $\mathrm{H})^{+}$; HR-ESI-MS obsd 590.29349, calcd 590.29329 [(M +H $\left.)^{+}, \mathrm{M}=\mathrm{C}_{27} \mathrm{H}_{39} \mathrm{~N}_{7} \mathrm{O}_{8}\right]$.

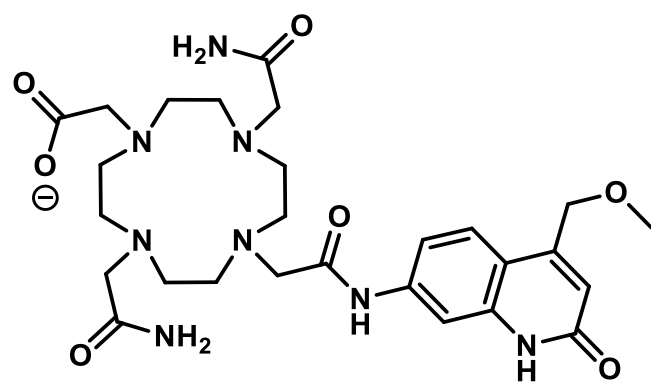

$\mathbf{L 3}^{\text {MOM }}$. A sample of $\mathbf{1 0}(347 \mathrm{mg}, 0.563 \mathrm{mmol})$ was suspended in $\mathrm{CH}_{3} \mathrm{CN}(4.8 \mathrm{~mL})$ and $\mathrm{H}_{2} \mathrm{O}$ (3.0 mL). Then aqueous $\mathrm{NaOH}$ solution $(1.0 \mathrm{M}, 1.8 \mathrm{~mL}, 1.8 \mathrm{mmol})$ was added. The mixture became a pale-yellow transparent solution. After 1 hour HPLC-MS analysis showed full cleavage of the ethyl ester. The reaction mixture was concentrated under reduced pressure and the crude product was dissolved in a few $\mathrm{mL}$ of $\mathrm{CH}_{3} \mathrm{CN}: \mathrm{H}_{2} \mathrm{O}$ equal mixture. The solution was loaded onto an alumina column. Elution with $\mathrm{CH}_{3} \mathrm{CN}: \mathrm{H}_{2} \mathrm{O}(100: 0 \rightarrow 70: 30)$ yielded a white solid (330 mg, quant.). ${ }^{1} \mathrm{H}$ NMR (400 MHz, DMSO-d6) $\delta$ ppm $1.74-4.23(\mathrm{~m}, 27 \mathrm{H},+1.8 \mathrm{H}$ $\mathrm{MeOH}), 4.51(\mathrm{~s}, 2 \mathrm{H}), 6.33(\mathrm{~s}, 1 \mathrm{H}), 7.10(\mathrm{~d}, J=8.2 \mathrm{~Hz}, 2 \mathrm{H}), 7.31(\mathrm{~d}, J=8.8 \mathrm{~Hz}, 1 \mathrm{H}), 7.44(\mathrm{~d}$, $J=1.5 \mathrm{~Hz}, 1 \mathrm{H}) ;{ }^{13} \mathrm{C}$ NMR $\left(101 \mathrm{MHz}, \mathrm{DMSO}-d_{6}\right) \delta \mathrm{ppm} 48.4,49.0,50.5,51.0,52.4,56.5,57.5$, 58.6, 70.1, 106.1, 114.5, 115.3, 115.7, 124.4, 137.8, 139.5, 148.9, 164.0, 170.9, 171.8, 176.1; RP-HPLC $t_{R}=2.63 \min \left(16 \min\right.$ method, $0-6 \min : 10 \%$ iso $\left.\mathrm{CH}_{3} \mathrm{CN} \& 6-12 \min : 10 \% \rightarrow 50 \%\right)$; ESI-MS obsd 589.82, calcd 589.32 (M + H) ${ }^{+}$; HR-ESI-MS obsd 611.29091, calcd 611.29122 $\left[(\mathrm{M}+\mathrm{Na})^{+}, \mathrm{M}=\mathrm{C}_{27} \mathrm{H}_{40} \mathrm{~N}_{8} \mathrm{O}_{7}\right]$

General procedure for synthesis of $\mathbf{L 4 a} a^{M e}, \mathbf{L A a}^{\mathrm{MOM}}$ and $\mathbf{L 4 a ^ { C F 3 }}$. The appropriate monoalkylated cyclen $\mathbf{1 1}^{\mathrm{Me}}, \mathbf{1 1}^{\mathrm{MOM}}$ or $\mathbf{1 1}^{\mathrm{CF} 3}$ (1 equiv.) was dissolved in DMF (70 mM). The base DIPEA (6 equiv.) was added, followed by the addition of 2-bromoacetamide (4a, 3.2 
equiv.). The reaction mixture was stirred at room temperature overnight. Next day the full conversion of the starting material to the target product was confirmed by $\mathrm{LC}-\mathrm{MS}$ and $\mathrm{CH}_{2} \mathrm{Cl}_{2}$ was added to the reaction mass. The formed precipitate was filtered, and the filter cake was washed with $\mathrm{CH}_{3} \mathrm{CN}: \mathrm{Et}_{2} \mathrm{O}$ (4:1) mixture. The products were obtained as white solids.

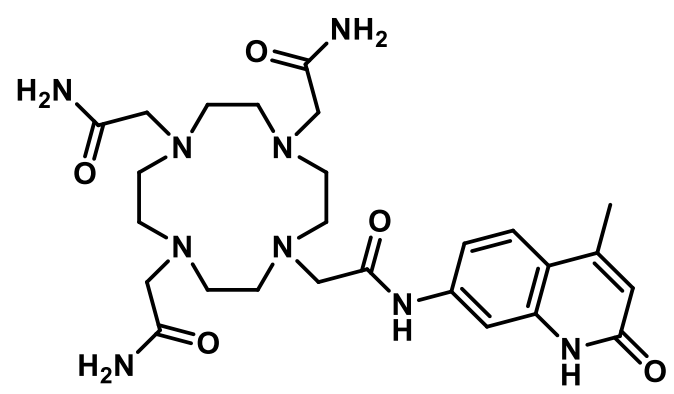

L4a ${ }^{\text {Me }}$. Known compound, ${ }^{3}$ full characterization. $206 \mathrm{mg}(49 \%) ;{ }^{1} \mathrm{H}$ NMR (400 MHz, DMSO- $\left.d_{6}\right) \delta$ ppm 2.37 (s, 3H), 2.55-3.50 (m, 30H (24H + 6H solvent residuals)), $6.25(\mathrm{~s}, 1 \mathrm{H})$, 6.73-8.01 (m, 9H), $10.10(\mathrm{~s}, 1 \mathrm{H}), 11.55(\mathrm{~s}, 1 \mathrm{H}) ;{ }^{13} \mathrm{C}$ NMR (101 MHz, DMSO- $\left.d_{6}\right) \delta \mathrm{ppm} \mathrm{18.4,}$ 53.0, 53.1, 53.2, 53.3, 57.8, 58.1, 58.5, 104.6, 113.7, 115.6, 119.0, 125.3, 139.5, 140.5, 147.7, 162.1 $170.0,172.9$; RP-HPLC $t_{\mathrm{R}}=1.18 \min (16 \min$ method, $0-8 \mathrm{~min}: 10 \rightarrow 20 \% \& 8-12 \mathrm{~min}:$ $20 \%$ iso $\left.\mathrm{CH}_{3} \mathrm{CN}\right)$; ESI-MS obsd 558.29, calcd 558.31 (M + H) ${ }^{+}$; HR-ESI-MS obsd 298.6364, calcd 298.6389 $\left[(\mathrm{M}+\mathrm{K}+\mathrm{H})^{2+}, \mathrm{M}=\mathrm{C}_{26} \mathrm{H}_{39} \mathrm{~N}_{9} \mathrm{O}_{5}\right]$.

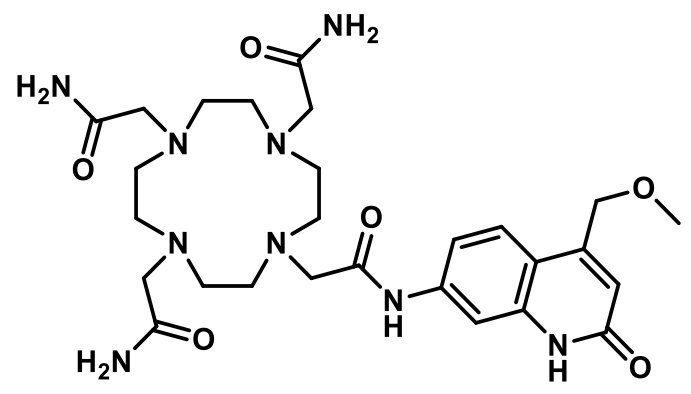

$\mathbf{L 4 a}^{\text {MOM }}$. $1.224 \mathrm{~g}(74 \%) ;{ }^{1} \mathrm{H}$ NMR (400 MHz, DMSO- $\left.d_{6}\right) \delta \mathrm{ppm} 2.52-3.13(\mathrm{~m}, 24 \mathrm{H},+3.3 \mathrm{H}$ DMF), 3.14-3.50 (m, 3H, + 5.6H H2 $\mathrm{O}, \mathrm{MeOH}), 4.62$ (s, 2H), 6.37 (s, 1H), 6.91 (br, 3H, NH), $7.35\left(\mathrm{dd}, J_{l}=8.8 \mathrm{~Hz}, J_{2}=1.8 \mathrm{~Hz}, 1 \mathrm{H}\right), 7.53(\mathrm{~s}, 3 \mathrm{H}, \mathrm{NH}), 7.59(\mathrm{~d}, J=8.8 \mathrm{~Hz}, 1 \mathrm{H}), 7.81(\mathrm{~d}, J=$ $1.7 \mathrm{~Hz}, 1 \mathrm{H}), 10.14(\mathrm{~s}, 1 \mathrm{H}), 11.66(\mathrm{~s}, 1 \mathrm{H}) ;{ }^{13} \mathrm{C} \mathrm{NMR}\left(101 \mathrm{MHz}, \mathrm{DMSO}-d_{6}\right) \delta \mathrm{ppm} 30.8,35.8$, 
53.1, 53.3, 57.8, 58.1, 58.1, 70.2, 104.7, 113.4, 113.7, 117.2, 124.7, 139.7, 140.5, 147.0, 162.0, 162.3, 170.5, 172.8; RP-HPLC $t_{\mathrm{R}}=1.17 \mathrm{~min}(16 \mathrm{~min}$ method, $0-8 \mathrm{~min}: 10 \rightarrow 20 \% \& 8-12 \mathrm{~min}:$ $20 \%$ iso $\left.\mathrm{CH}_{3} \mathrm{CN}\right)$; ESI-MS obsd 588.39, calcd 588.33 $(\mathrm{M}+\mathrm{H})^{+}$; HR-ESI-MS obsd 313.6423, calcd $313.6442\left[(\mathrm{M}+\mathrm{K}+\mathrm{H})^{2+}, \mathrm{M}=\mathrm{C}_{27} \mathrm{H}_{41} \mathrm{~N}_{9} \mathrm{O}_{6}\right]$.

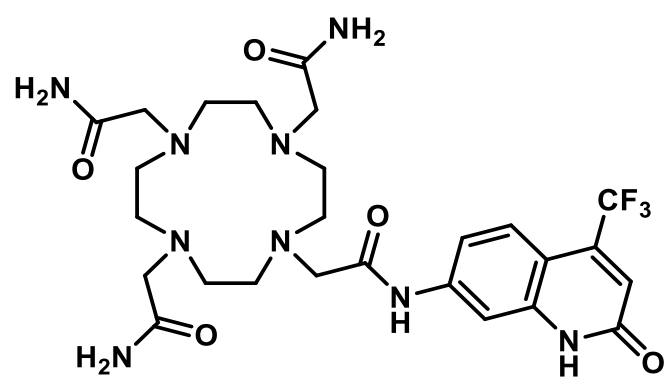

$\mathbf{L 4 a}^{\text {CF3 }} .257 \mathrm{mg}$ (74\%); ${ }^{1} \mathrm{H}$ NMR (400 MHz, DMSO- $\left.d_{6}\right) \delta$ ppm 2.57-4.18 (m, 30H (24H + 6H solvent residue), $6.81(\mathrm{~s}, 1 \mathrm{H}), 7.06-7.85(\mathrm{~m}, 8 \mathrm{H}), 7.99(\mathrm{~s}, 1 \mathrm{H}), 10.35(\mathrm{~s}, 1 \mathrm{H}), 12.23(\mathrm{~s}, 1 \mathrm{H})$; ${ }^{13} \mathrm{C}$ NMR (101 MHz, DMSO-d $) \delta$ ppm 50.3, 50.6, 51.5, 52.0, 55.8, 55.9, 57.4, 105.2, 108.9, $115.1,119.4,122.60$ (q, $J=274.8 \mathrm{~Hz}), 125.0,136.3(\mathrm{q}, J=30.8 \mathrm{~Hz}), 140.7,141.5,160.4,169.6$, 170.5, 171.0; ${ }^{19}$ F NMR (376 MHz, DMSO- $\left.d_{6}\right) \delta \mathrm{ppm}-62.4 ; \mathrm{RP}-\mathrm{HPLC} \mathrm{t}_{\mathrm{R}}=1.52 \min (16 \mathrm{~min}$ method, $0-8$ min: $10 \rightarrow 20 \% \& 8-12$ min: $20 \%$ iso $\mathrm{CH}_{3} \mathrm{CN}$ ); ESI-MS obsd 612.29, calcd 612.29 $(\mathrm{M}+\mathrm{H})^{+} ;$HR-ESI-MS obsd 370.6766, calcd $370.6782\left[(\mathrm{M}+\mathrm{K}+\mathrm{H})^{2+}, \mathrm{M}=\mathrm{C}_{26} \mathrm{H}_{36} \mathrm{~N}_{9} \mathrm{O}_{5} \mathrm{~F}_{3}\right]$.

General procedure for synthesis of $\mathbf{L} 4 \mathbf{b}, \mathbf{c}^{\mathrm{MOM}}$. The antenna-functionalized cyclen (11 $\left.{ }^{\text {MOM }}\right)$ was dissolved in DMF (72 $\left.\mathrm{mM}\right)$ and DIPEA (5 equiv.) was added to the solution. The reaction mixture was stirred at room temperature overnight after the addition of 3.2 equivalents of 2-bromo-N-methylacetamide (4b) or 2-bromo-N,N-dimethylacetamide (4c) for the preparation of $\mathbf{L} \mathbf{4} b^{\text {MOM }}$ or $\mathbf{L} 4 c^{\text {MOM }}$ respectively. On the following day the LC-MS analysis showed full conversion of the starting reagent to the product and the reaction mass was directly loaded on neutral alumina column which had been conditioned with $100 \% \mathrm{CH}_{3} \mathrm{CN}$. Elution with $\mathrm{CH}_{3} \mathrm{CN}: \mathrm{H}_{2} \mathrm{O}(100: 0 \rightarrow 70: 30$, low gradient $)$ yielded the product of high purity. 


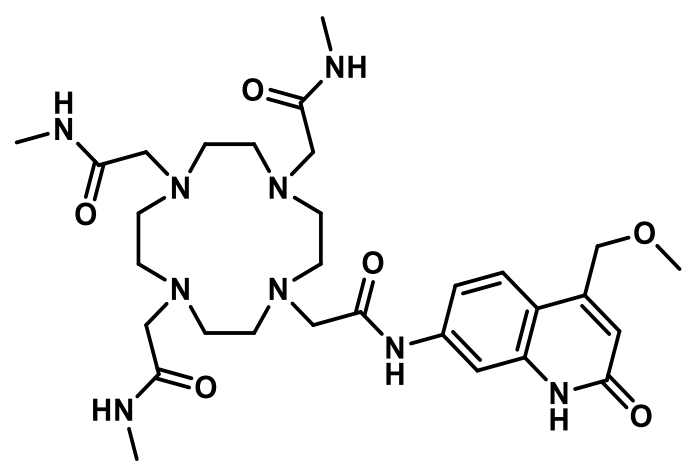

L4b ${ }^{\text {MOM }}$. 169 mg (74\%); ${ }^{1} \mathrm{H}$ NMR (400 MHz, DMSO-d $) \delta$ ppm 2.25-3.01 (m, 27H), 3.30$3.38(\mathrm{~m}, 9 \mathrm{H}), 4.62(\mathrm{~s}, 2 \mathrm{H}), 6.36(\mathrm{~s}, 1 \mathrm{H}), 7.50\left(\mathrm{dd}, J_{l}=8.9 \mathrm{~Hz}, J_{2}=1.5 \mathrm{~Hz}, 1 \mathrm{H}\right), 7.58(\mathrm{~d}, J=8.9$ $\mathrm{Hz}, 1 \mathrm{H}), 7.62(\mathrm{~d}, J=1.4 \mathrm{~Hz}, 1 \mathrm{H}), 8.16(\mathrm{~s}, 2 \mathrm{H}), 8.17(\mathrm{~s}, 1 \mathrm{H}), 10.59(\mathrm{~s}, 1 \mathrm{H}), 11.73(\mathrm{br}, 1 \mathrm{H}) ;{ }^{13} \mathrm{C}$ NMR (101 MHz, DMSO-d6) $\delta$ ppm 25.4, 25.4, 50.4, 56.7, 57.0, 58.1, 70.2, 105.0, 113.4, 114.2, $117.2,124.6,139.6,140.6,147.0,161.9,162.3,170.6,171.4,171.5 ; \mathrm{RP}-H P L C t_{R}=2.40 \mathrm{~min}$ (16 min method, 0-6 min: $10 \%$ iso $\mathrm{CH}_{3} \mathrm{CN} \&$ 6-12 $\mathrm{min}: 10 \% \rightarrow 50 \%$ ); ESI-MS obsd 630.68, calcd $630.37(\mathrm{M}+\mathrm{H})^{+}$; HR-ESI-MS obsd 652.3559, calcd $652.3542\left[(\mathrm{M}+\mathrm{Na})^{+}, \mathrm{M}=\right.$ $\left.\mathrm{C}_{30} \mathrm{H}_{47} \mathrm{~N}_{9} \mathrm{O}_{6}\right]$.

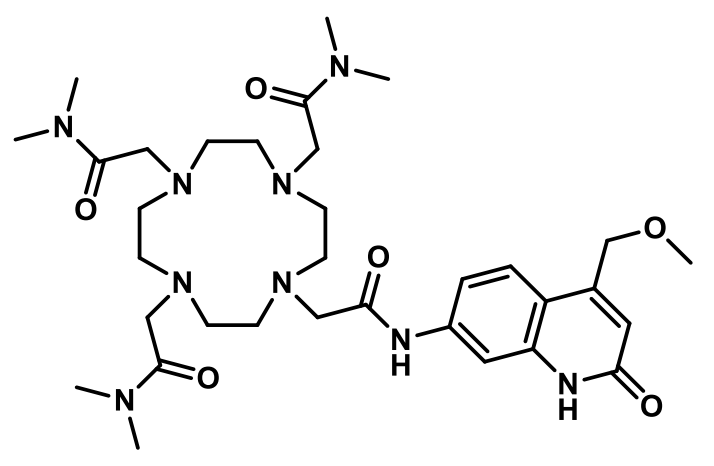

L4c ${ }^{\text {MOM }} .142 \mathrm{mg}(59 \%) ;{ }^{1} \mathrm{H}$ NMR (400 MHz, DMSO-d $)_{6} \delta \mathrm{ppm} 1.98-3.55(\mathrm{~s}, 45 \mathrm{H},+7.2 \mathrm{H}$ DMSO, $\left.\mathrm{H}_{2} \mathrm{O}\right), 4.64(\mathrm{~s}, 2 \mathrm{H}), 6.37(\mathrm{~s}, 1 \mathrm{H}), 7.49(\mathrm{~s}, 1 \mathrm{H}), 7.55-7.60(\mathrm{~m}, 2 \mathrm{H}), 10.68(\mathrm{~s}, 1 \mathrm{H}), 11.71$ $(\mathrm{s}, 1 \mathrm{H}) ;{ }^{13} \mathrm{C}$ NMR (101 MHz, DMSO-d $) \delta \mathrm{ppm} 35.0,35.1,35.6,35.8$. 49.7-51.6, 54.6, 57.3, $58.1,70.2,104.8,113.5,114.0,117.2,124.5,139.6,140.7,147.0,161.9,170.9,171.0$; RPHPLC $t_{R}=2.52,4.15 \min \left(0-6 \min : 10 \%\right.$ iso $\left.\mathrm{CH}_{3} \mathrm{CN} \& 6-12 \mathrm{~min}: 10 \% \rightarrow 50 \%\right)$; ESI-MS obsd 
672.60, calcd 672.42 $(\mathrm{M}+\mathrm{H})^{+}$. HR-ESI-MS obsd 694.4031, calcd 694.4011 [(M + Na) ${ }^{+}, \mathrm{M}=$ $\left.\mathrm{C}_{33} \mathrm{H}_{53} \mathrm{~N}_{9} \mathrm{O}_{6}\right]$.

General procedure for Ln (III) complexation with $\mathrm{L2}^{\mathrm{MOM}}, \mathrm{L3}^{\mathrm{MOM}}, \mathrm{L4a-cMOM}$ and $\mathbf{L 4} \mathbf{a}^{\text {Me/CF3 }}$. A sample of the appropriate ligand (1 equiv.) in the mixture with the corresponding anhydrous $\mathrm{LnCl}_{3}$ (2.4 equiv.) were dissolved in $\mathrm{H}_{2} \mathrm{O}: \mathrm{EtOH}$ equal mixture (0.05 M). The reaction solution was stirred at $45{ }^{\circ} \mathrm{C}$ for $24 \mathrm{~h}$. The completion of the complexation was observed via LC-MS and TLC analysis. Column chromatography on neutral alumina (Isopropanol: $\mathrm{H}_{2} \mathrm{O}, 9: 1 \rightarrow 6: 4$; Isopropanol:EtOH: $\mathrm{H}_{2} \mathrm{O}, 4: 2: 4 \rightarrow 2: 4: 4$ ) was performed on the reaction mixtures yielding colorless complexes. The isolated compounds contain traces of initial lanthanide chlorides.

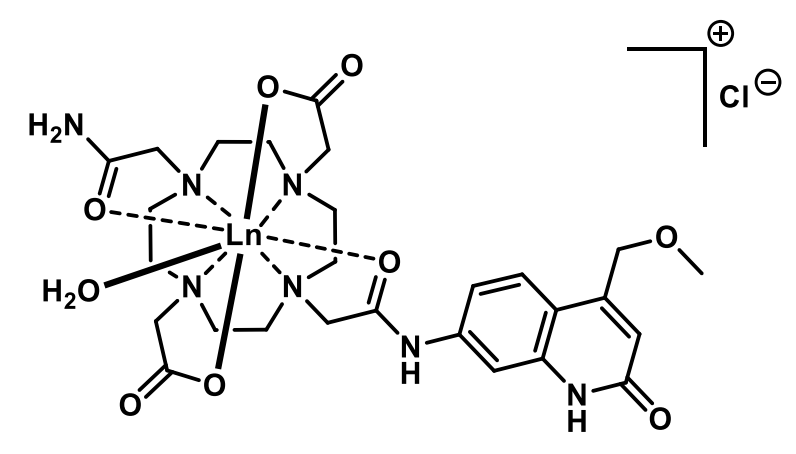

EuL2 ${ }^{\text {MOM }} .24 \mathrm{mg}(61 \%)$; RP-HPLC $t_{R}=2.72$ min 16 min method, $0-6$ min: $10 \%$ iso $\mathrm{CH}_{3} \mathrm{CN}$ \& 6-12 min: $10 \% \rightarrow 50 \%)$; ESI-MS obsd 741.53, calcd $741.20(\mathrm{M}+\mathrm{H})^{+}$; HR-ESI-MS obsd 740.19080, calcd 740.19127 [(M)+, $\left.\mathrm{M}=\mathrm{C}_{27} \mathrm{H}_{37} \mathrm{~N}_{7} \mathrm{O}_{8} \mathrm{Eu}\right] ; \lambda_{\mathrm{em}}=374,579,588,592,596,613$, $617,623,653,682,688,694,700,752,760 \mathrm{~nm}\left(\lambda_{\mathrm{ex}}=329.5 \mathrm{~nm}\right)$.

GdL2 $^{\text {MOM }}$. $45 \mathrm{mg}$ (quant.); RP-HPLC $t_{\mathrm{R}}=2.67 \mathrm{~min} 16 \mathrm{~min}$ method, $0-6 \mathrm{~min}: 10 \%$ iso $\left.\mathrm{CH}_{3} \mathrm{CN} \& 6-12 \mathrm{~min}: 10 \% \rightarrow 50 \%\right)$; ESI-MS obsd 746.55 , calcd $746.21(\mathrm{M}+2 \mathrm{H})^{2+}$; HR-ESIMS obsd 745.19515, calcd 745.19454 [(M)+, $\left.\mathrm{M}=\mathrm{C}_{27} \mathrm{H}_{37} \mathrm{~N}_{7} \mathrm{O}_{8} \mathrm{Gd}\right] ; \lambda_{\mathrm{em}}=376 \mathrm{~nm}\left(\lambda_{\mathrm{ex}}=329.5\right.$ $\mathrm{nm})$. 
TbL2 $^{\text {MOM }}$. $61 \mathrm{mg}$ (quant.); RP-HPLC $\mathrm{t}_{\mathrm{R}}=2.70 \mathrm{~min} 16 \mathrm{~min}$ method, $0-6 \mathrm{~min}: 10 \%$ iso $\mathrm{CH}_{3} \mathrm{CN} \&$ 6-12 min: $\left.10 \% \rightarrow 50 \%\right)$; ESI-MS obsd 746.90, calcd 746.20 (M + H) ${ }^{+} ;$HR-ESI-MS obsd 746.19461, calcd 746.19516 [(M)+, M = $\left.\mathrm{C}_{27} \mathrm{H}_{37} \mathrm{~N}_{7} \mathrm{O}_{8} \mathrm{~Tb}\right] ; \lambda_{\mathrm{em}}=375,487,545,587,620$, $640,649,666,682 \mathrm{~nm}\left(\lambda_{\mathrm{ex}}=329.5 \mathrm{~nm}\right)$.

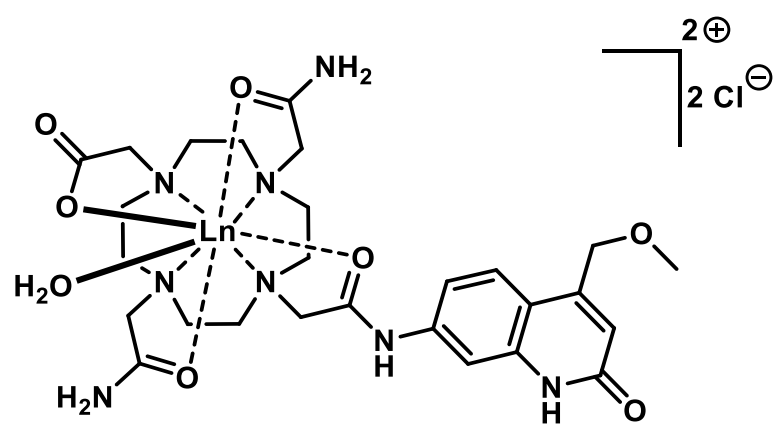

EuL3 $^{\text {MOM }}$. $67 \mathrm{mg}$ (quant.); RP-HPLC $t_{\mathrm{R}}=2.42 \mathrm{~min} 16 \mathrm{~min}$ method, $0-6 \mathrm{~min}: 10 \%$ iso $\mathrm{CH}_{3} \mathrm{CN} \&$ 6-12 min: $\left.10 \% \rightarrow 50 \%\right)$; ESI-MS obsd 371.06, calcd $371.12(\mathrm{M}+\mathrm{H})^{3+}$; HR-ESI-MS obsd 370.10711, calcd 370.10726 [(M $\left.{ }^{2+}, \mathrm{M}=\mathrm{C}_{27} \mathrm{H}_{39} \mathrm{~N}_{8} \mathrm{O} 7 \mathrm{Eu}\right] ; \lambda_{\mathrm{em}}=376,579,589,594,615$, $623,653,682,687,694,700,752,761 \mathrm{~nm}\left(\lambda_{\mathrm{ex}}=329.5 \mathrm{~nm}\right)$.

GdL3 $^{\text {MOM }}$. $63 \mathrm{mg}$ (quant.); RP-HPLC $t_{\mathrm{R}}=2.28 \mathrm{~min} 16 \mathrm{~min}$ method, $0-6 \mathrm{~min}: 10 \%$ iso $\left.\mathrm{CH}_{3} \mathrm{CN} \& 6-12 \min : 10 \% \rightarrow 50 \%\right)$; ESI-MS obsd 373.29, calcd $373.62(\mathrm{M}+\mathrm{H})^{3+} ;$ HR-ESI-MS obsd 372.60873, calcd 372.60890 [(M $\left.)^{2+}, \mathrm{M}=\mathrm{C}_{27} \mathrm{H}_{39} \mathrm{~N}_{8} \mathrm{O}_{7} \mathrm{Gd}\right] ; \lambda_{\mathrm{em}}=375 \mathrm{~nm}\left(\lambda_{\mathrm{ex}}=329.5 \mathrm{~nm}\right)$.

TbL3 $^{\text {MOM }} .68 \mathrm{mg}$ (quant.); RP-HPLC $\mathrm{t}_{\mathrm{R}}=2.23 \mathrm{~min} 16 \mathrm{~min}$ method, $0-6 \mathrm{~min}: 10 \%$ iso $\mathrm{CH}_{3} \mathrm{CN} \&$ 6-12 min: $\left.10 \% \rightarrow 50 \%\right)$; ESI-MS obsd 374.04, calcd $374.12(\mathrm{M}+\mathrm{H})^{3+}$; HR-ESI-MS obsd 373.10910, calcd 373.10921 [(M $\left.)^{2+}, \mathrm{M}=\mathrm{C}_{27} \mathrm{H}_{39} \mathrm{~N}_{8} \mathrm{O}_{7} \mathrm{~Tb}\right] ; \lambda_{\mathrm{em}}=376,487,546,582,587$, $620,640,650,667,680 \mathrm{~nm}\left(\lambda_{\mathrm{ex}}=329.5 \mathrm{~nm}\right)$. 


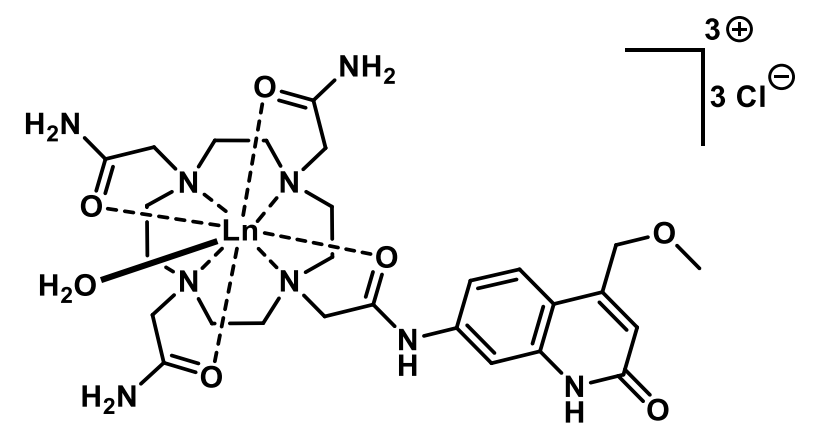

EuL4a $^{\text {MOM }}$. $191 \mathrm{mg}(95 \%)$; RP-HPLC $t_{\mathrm{R}}=2.25 \mathrm{~min} 16 \mathrm{~min}$ method, 0-6 min: $10 \%$ iso $\mathrm{CH}_{3} \mathrm{CN} \&$ 6-12 min: $\left.10 \% \rightarrow 50 \%\right)$; ESI-MS obsd 392.87, calcd $393.11(\mathrm{M}+\mathrm{Na})^{3+}$; HR-ESIMS obsd 246.74620, calcd $246.74593\left[(\mathrm{M})^{3+}, \mathrm{M}=\mathrm{C}_{27} \mathrm{H}_{41} \mathrm{~N}_{9} \mathrm{O}_{6} \mathrm{Eu}\right] ; \lambda_{\mathrm{em}}=375,579,589,594$, $615,623,653,682,687,695,699 \mathrm{~nm}\left(\lambda_{\mathrm{ex}}=329.5 \mathrm{~nm}\right)$.

GdL4a $^{\text {MOM }}$. $41.5 \mathrm{mg}$ (96\%); RP-HPLC $\mathrm{t}_{\mathrm{R}}=2.28 \mathrm{~min} 16 \mathrm{~min}$ method, $0-6 \mathrm{~min}: 10 \%$ iso $\mathrm{CH}_{3} \mathrm{CN} \&$ 6-12 min: 10\% $\rightarrow 50 \%$ ); ESI-MS obsd 373.29, calcd $373.63(\mathrm{M}+\mathrm{H})^{3+} ;$ HR-ESI-MS obsd 248.41346, calcd 248.41368 [(M $\left.{ }^{3+}, \mathrm{M}=\mathrm{C}_{27} \mathrm{H}_{41} \mathrm{~N}_{9} \mathrm{O}_{6} \mathrm{Gd}\right] ; \lambda_{\mathrm{em}}=376 \mathrm{~nm}\left(\lambda_{\mathrm{ex}}=329.5 \mathrm{~nm}\right)$.

TbL4a ${ }^{\text {MOM }}$. $40.9 \mathrm{mg}(94 \%)$; RP-HPLC $\mathrm{t}_{\mathrm{R}}=2.22 \mathrm{~min} 16 \mathrm{~min}$ method, $0-6 \mathrm{~min}: 10 \%$ iso $\mathrm{CH}_{3} \mathrm{CN} \&$ 6-12 min: $\left.10 \% \rightarrow 50 \%\right)$; ESI-MS obsd 397.36, calcd $397.12(\mathrm{M}+\mathrm{Na}+\mathrm{H})^{4+}$; HRESI-MS obsd 248.74698, calcd 248.74723 [(M) $\left.{ }^{3+}, \mathrm{M}=\mathrm{C}_{27} \mathrm{H}_{41} \mathrm{~N}_{9} \mathrm{O}_{6} \mathrm{~Tb}\right] ; \lambda_{\mathrm{em}}=375,487,545$, $582,587,620,640,650,667,680 \mathrm{~nm}\left(\lambda_{\mathrm{ex}}=329.5 \mathrm{~nm}\right)$.

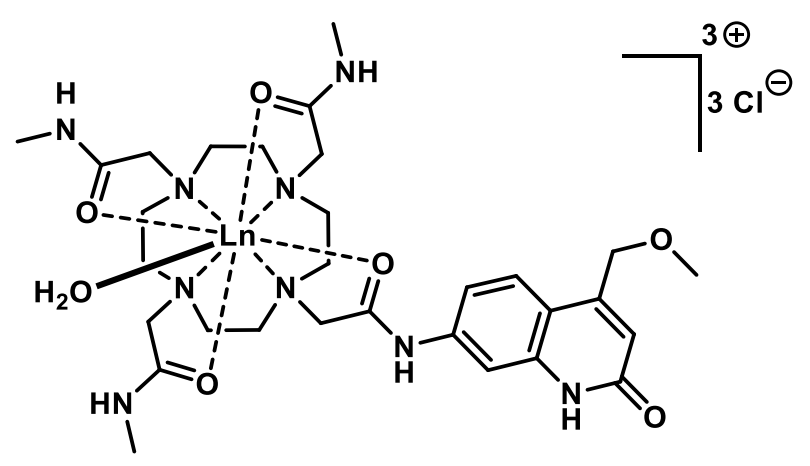

EuL4b $^{\text {MOM }}$. $36 \mathrm{mg}$ (quant.); RP-HPLC $\mathrm{t}_{\mathrm{R}}=2.23 \mathrm{~min} 16 \mathrm{~min}$ method, $0-6 \mathrm{~min}: 10 \%$ iso $\mathrm{CH}_{3} \mathrm{CN} \&$ 6-12 min: $\left.10 \% \rightarrow 50 \%\right)$; ESI-MS obsd 415.23, calcd $415.14(\mathrm{M}+\mathrm{Na}+\mathrm{H})^{4+}$; HR- 
ESI-MS obsd 390.63842, calcd $390.63875\left[(\mathrm{M}-\mathrm{H})^{2+}, \mathrm{M}=\mathrm{C}_{30} \mathrm{H}_{47} \mathrm{~N}_{9} \mathrm{O}_{6} \mathrm{Eu}\right] ; \lambda_{\mathrm{em}}=374,579$, $589,594,615,622,653,682,687,695,699,751,761 \mathrm{~nm}\left(\lambda_{\mathrm{ex}}=335 \mathrm{~nm}\right)$.

GdL4b ${ }^{\text {MOM }} .22 \mathrm{mg}(62 \%)$; RP-HPLC $\mathrm{t}_{\mathrm{R}}=2.25 \mathrm{~min} 16 \mathrm{~min}$ method, $0-6 \mathrm{~min}: 10 \%$ iso $\mathrm{CH}_{3} \mathrm{CN} \&$ 6-12 min: $\left.10 \% \rightarrow 50 \%\right)$; ESI-MS obsd 418.26, calcd $418.64(\mathrm{M}+\mathrm{Na}+2 \mathrm{H})^{5+}$; HRESI-MS obsd 262.42970, calcd 262.42936 [(M) $\left.{ }^{3+}, \mathrm{M}=\mathrm{C}_{30} \mathrm{H}_{47} \mathrm{~N}_{9} \mathrm{O}_{6} \mathrm{Gd}\right] ; \lambda_{\mathrm{em}}=375 \mathrm{~nm}\left(\lambda_{\mathrm{ex}}=\right.$ $329.5 \mathrm{~nm})$.

TbL4b $^{\text {MOM }} .37 \mathrm{mg}$ (quant.); RP-HPLC $\mathrm{t}_{\mathrm{R}}=2.23 \mathrm{~min} 16 \mathrm{~min}$ method, $0-6 \mathrm{~min}: 10 \%$ iso $\mathrm{CH}_{3} \mathrm{CN} \&$ 6-12 min: $\left.10 \% \rightarrow 50 \%\right)$; ESI-MS obsd 417.55, calcd $417.13(\mathrm{M}+\mathrm{Na})^{3+}$; HR-ESIMS obsd 393.64037, calcd 393.64068 $\left[(\mathrm{M}-\mathrm{H})^{2+}, \mathrm{M}=\mathrm{C}_{30} \mathrm{H}_{47} \mathrm{~N}_{9} \mathrm{O}_{6} \mathrm{~Tb}\right] ; \lambda_{\mathrm{em}}=376,487,545$, $582,587,620,640,650,667,680 \mathrm{~nm}\left(\lambda_{\mathrm{ex}}=329.5 \mathrm{~nm}\right)$.

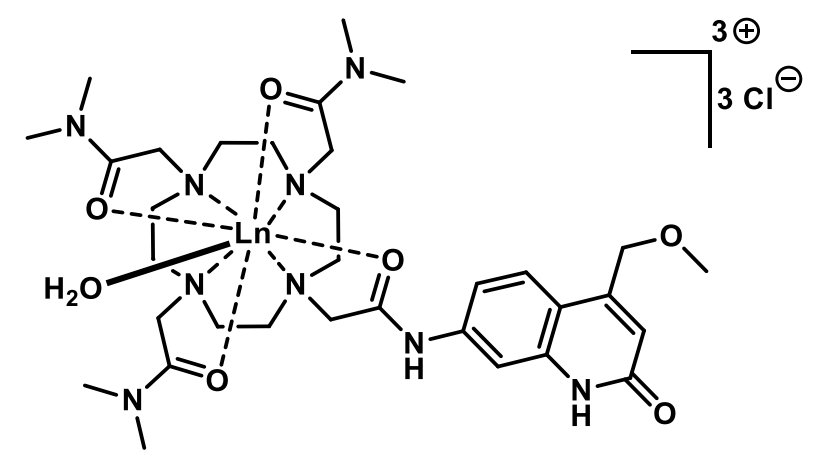

EuL4c ${ }^{\text {MOM }} .36$ mg (quant.); RP-HPLC $t_{R}=2.30 \mathrm{~min} 16 \mathrm{~min}$ method, $0-6 \mathrm{~min}: 10 \%$ iso $\mathrm{CH}_{3} \mathrm{CN} \&$ 6-12 min: $\left.10 \% \rightarrow 50 \%\right)$; ESI-MS obsd 435.10, calcd $435.15(\mathrm{M}+\mathrm{Na})^{3+}$; HR-ESIMS obsd 411.66183, calcd 411.66225 [(M - H $\left.)^{2+}, \mathrm{M}=\mathrm{C}_{33} \mathrm{H}_{53} \mathrm{~N}_{9} \mathrm{O}_{6} \mathrm{Eu}\right] ; \lambda_{\mathrm{em}}=375,579,589$, $593,615,623,653,682,688,695,700,751,761 \mathrm{~nm}\left(\lambda_{\mathrm{ex}}=335 \mathrm{~nm}\right)$.

GdL4c MOM $.27 \mathrm{mg}(77 \%)$; RP-HPLC $\mathrm{t}_{\mathrm{R}}=2.27 \mathrm{~min} 16 \mathrm{~min}$ method, $0-6 \mathrm{~min}: 10 \%$ iso $\mathrm{CH}_{3} \mathrm{CN} \& 6-12$ min: $\left.10 \% \rightarrow 50 \%\right)$; ESI-MS obsd 437.34, calcd $437.66(\mathrm{M}+\mathrm{Na})^{3+}$; HR-ESIMS obsd 276.44488, calcd $276.44503\left[(\mathrm{M})^{3+}, \mathrm{M}=\mathrm{C}_{33} \mathrm{H}_{53} \mathrm{~N}_{9} \mathrm{O}_{6} \mathrm{Gd}\right] ; \lambda_{\mathrm{em}}=376 \mathrm{~nm}\left(\lambda_{\mathrm{ex}}=329.5\right.$ $\mathrm{nm})$. 
TbL4c $\mathbf{c}^{\text {MOM }} .32 \mathrm{mg}(92 \%)$; RP-HPLC $\mathrm{t}_{\mathrm{R}}=2.32 \mathrm{~min} 16 \mathrm{~min}$ method, $0-6 \mathrm{~min}: 10 \%$ iso $\mathrm{CH}_{3} \mathrm{CN} \&$ 6-12 min: $\left.10 \% \rightarrow 50 \%\right)$; ESI-MS obsd 438.79, calcd $438.16(\mathrm{M}+\mathrm{Na})^{3+}$; HR-ESIMS obsd 414.66365, calcd 414.66415 [(M - H $\left.)^{2+}, \mathrm{M}=\mathrm{C}_{33} \mathrm{H}_{53} \mathrm{~N}_{9} \mathrm{O}_{6} \mathrm{~Tb}\right] ; \lambda_{\mathrm{em}}=375,487,542$, $546,581,587,620,640,650,667,681 \mathrm{~nm}\left(\lambda_{\mathrm{ex}}=329.5 \mathrm{~nm}\right)$.

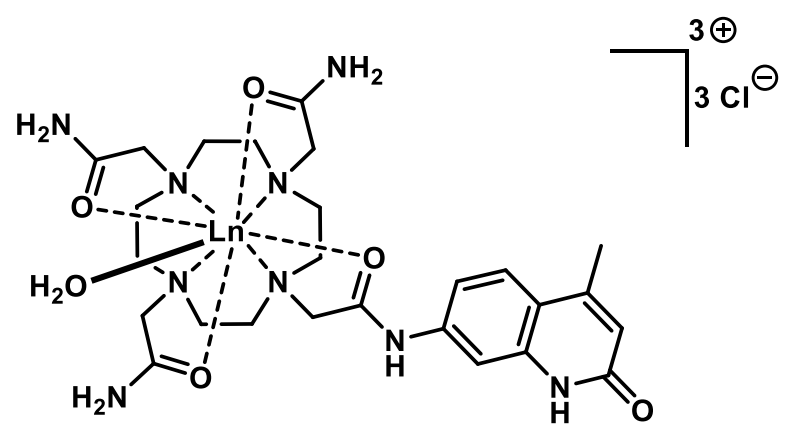

EuL4a $^{\text {Me }} .28 \mathrm{mg}(64 \%)$; RP-HPLC $t_{R}=1.12 \min (16 \min$ method, $0-8 \mathrm{~min}: 10 \rightarrow 20 \%$ \& $8-$ 12 min: $20 \%$ iso $\left.\mathrm{CH}_{3} \mathrm{CN}\right)$; ESI-MS obsd 377.18, calcd $377.10(\mathrm{M}+\mathrm{Na}-\mathrm{H})^{2+}$; HR-ESI-MS obsd 236.74212, calcd 236.74240 [(M $\left.)^{3+}, \mathrm{M}=\mathrm{C}_{26} \mathrm{H}_{39} \mathrm{~N}_{9} \mathrm{O}_{5} \mathrm{Eu}\right] ; \lambda_{\mathrm{em}}=366,579,589,594,615$, $623,653,682,687,699 \mathrm{~nm}\left(\lambda_{\mathrm{ex}}=308 \mathrm{~nm}\right)$.

GdL4a $^{\mathrm{Me}} .10 \mathrm{mg}(23 \%)$; RP-HPLC $\mathrm{t}_{\mathrm{R}}=1.13 \mathrm{~min}(16 \mathrm{~min}$ method, $0-8 \mathrm{~min}: 10 \rightarrow 20 \%$ \& $8-$ 12 min: $20 \%$ iso $\left.\mathrm{CH}_{3} \mathrm{CN}\right)$; ESI-MS obsd 379.77, calcd $379.60(\mathrm{M}+\mathrm{Na}-\mathrm{H})^{2+}$; HR-ESI-MS obsd 238.40992, calcd 238.41015 [(M) $\left.{ }^{3+}, \mathrm{M}=\mathrm{C}_{26} \mathrm{H}_{39} \mathrm{~N}_{9} \mathrm{O}_{5} \mathrm{Gd}\right] ; \lambda_{\mathrm{em}}=368 \mathrm{~nm}\left(\lambda_{\mathrm{ex}}=308 \mathrm{~nm}\right)$.

TbL4a $^{\text {Me }} .18 \mathrm{mg}(41 \%)$; RP-HPLC $t_{R}=1.12 \min (16 \min$ method, $0-8 \mathrm{~min}: 10 \rightarrow 20 \% \& 8-$ 12 min: $20 \%$ iso $\left.\mathrm{CH}_{3} \mathrm{CN}\right)$; ESI-MS obsd 380.42, calcd $380.10(\mathrm{M}+\mathrm{Na}-\mathrm{H})^{2+}$; HR-ESI-MS obsd 238.74346, calcd 238.74370 [(M) $\left.{ }^{3+}, \mathrm{M}=\mathrm{C}_{26} \mathrm{H}_{39} \mathrm{~N}_{9} \mathrm{O}_{5} \mathrm{~Tb}\right] ; \lambda_{\mathrm{em}}=368,487,545,582,587$, $620,640,650,667,680 \mathrm{~nm}\left(\lambda_{\mathrm{ex}}=307 \mathrm{~nm}\right)$. 


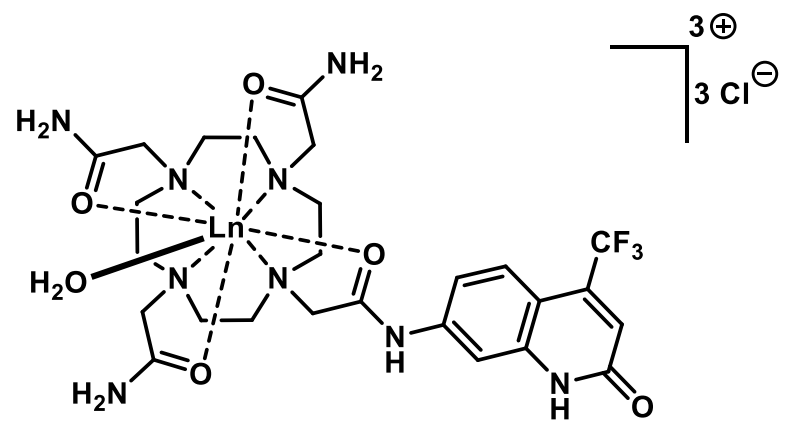

EuL4a ${ }^{\text {CF3 }} .12 \mathrm{mg}(28 \%)$; RP-HPLC $\mathrm{t}_{\mathrm{R}}=1.22 \mathrm{~min}(16 \mathrm{~min}$ method, $0-8 \mathrm{~min}: 10 \rightarrow 20 \%$ \& 8-12 min: 20\% iso $\left.\mathrm{CH}_{3} \mathrm{CN}\right)$; ESI-MS obsd 404.57, calcd 404.08 $(\mathrm{M}+\mathrm{Na}-\mathrm{H})^{2+} ;$ HR-ESI-MS obsd 254.73300, calcd 254.73298 [(M $\left.)^{3+}, \mathrm{M}=\mathrm{C}_{26} \mathrm{H}_{36} \mathrm{~N}_{9} \mathrm{O}_{5} \mathrm{~F}_{3} \mathrm{Eu}\right] ; \lambda_{\mathrm{em}}=393,579,590,594$, $615,623,653,682,687,699 \mathrm{~nm}\left(\lambda_{\mathrm{ex}}=315 \mathrm{~nm}\right)$.

GdL4a $^{\text {CF3 }} .16 \mathrm{mg}(38 \%)$; RP-HPLC $t_{R}=1.20 \mathrm{~min}(16 \mathrm{~min}$ method, $0-8 \mathrm{~min}: 10 \rightarrow 20 \%$ \& 8-12 min: $20 \%$ iso $\left.\mathrm{CH}_{3} \mathrm{CN}\right)$; ESI-MS obsd 405.92, calcd $405.56(\mathrm{M}+\mathrm{Na}-2 \mathrm{H})^{+}$; HR-ESI-MS obsd 256.40052, calcd 256.40073 [(M) $\left.{ }^{3+}, \mathrm{M}=\mathrm{C}_{26} \mathrm{H}_{36} \mathrm{~N}_{9} \mathrm{O}_{5} \mathrm{~F}_{3} \mathrm{Gd}\right] ; \lambda_{\mathrm{em}}=391 \mathrm{~nm}\left(\lambda_{\mathrm{ex}}=315 \mathrm{~nm}\right)$.

TbL4a ${ }^{\text {CF3 }} .34 \mathrm{mg}(79 \%)$; RP-HPLC $t_{\mathrm{R}}=1.18 \mathrm{~min}(16 \mathrm{~min}$ method, $0-8 \mathrm{~min}: 10 \rightarrow 20 \%$ \& 8-12 min: 20\% iso $\left.\mathrm{CH}_{3} \mathrm{CN}\right)$; ESI-MS obsd 407.55, calcd $407.08(\mathrm{M}+\mathrm{Na}-\mathrm{H})^{2+}$; HR-ESI-MS obsd 256.73416, calcd 256.73428 [(M $\left.)^{3+}, \mathrm{M}=\mathrm{C}_{26} \mathrm{H}_{36} \mathrm{~N}_{9} \mathrm{O}_{5} \mathrm{~F}_{3} \mathrm{~Tb}\right] ; \lambda_{\mathrm{em}}=391,487,545,582$, $587,620,641,650,668,680 \mathrm{~nm}\left(\lambda_{\mathrm{ex}}=315 \mathrm{~nm}\right)$. 


\section{${ }^{1} \mathrm{H}$ NMR spectra of Eu(III) complexes}

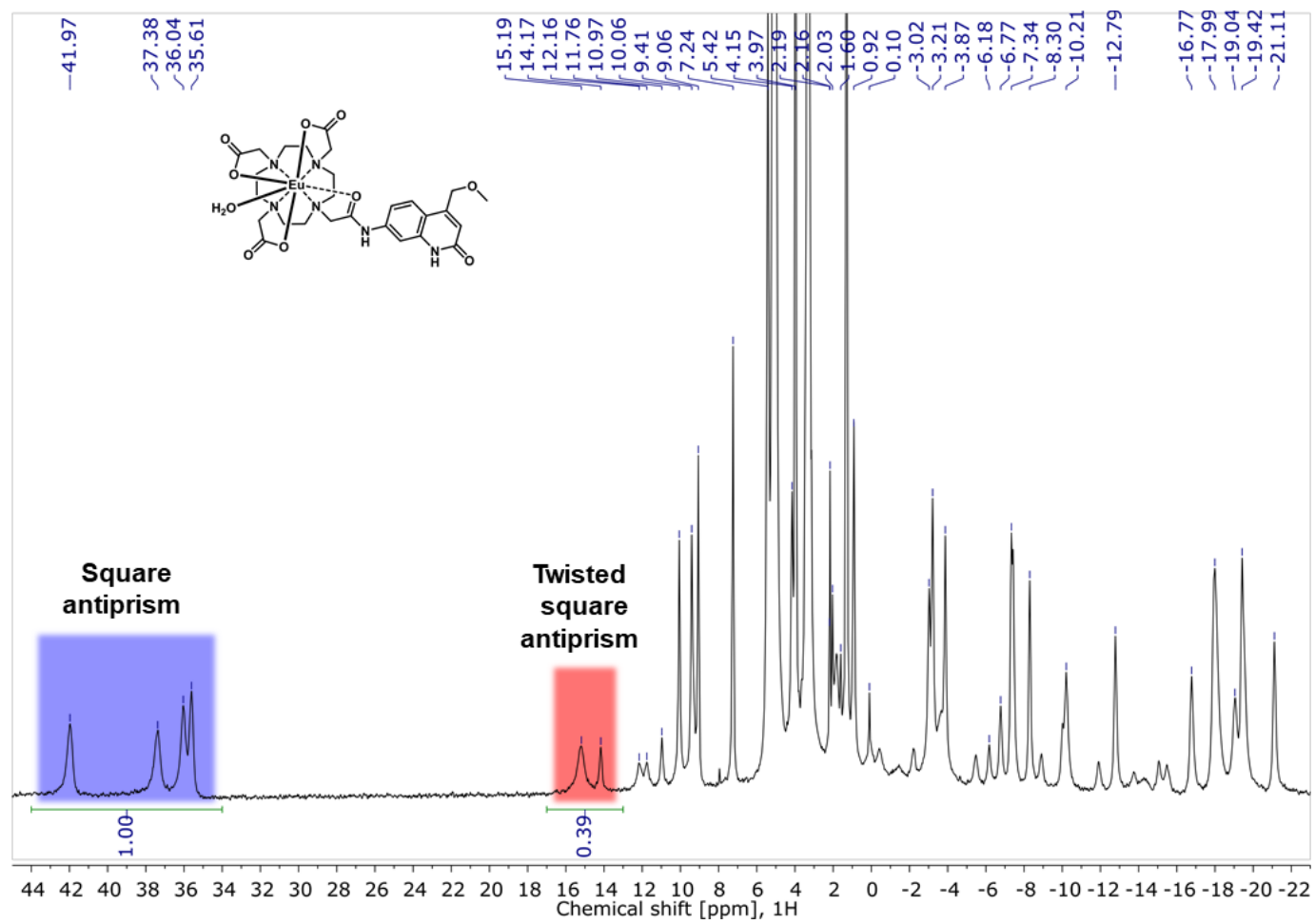

Figure S1. ${ }^{1} \mathrm{H}$ NMR spectrum of EuL1 ${ }^{\text {MOM }}\left(400 \mathrm{MHz}, \mathrm{CD}_{3} \mathrm{OD}, 0{ }^{\circ} \mathrm{C}\right.$ ) with the regions corresponding to the SAP and TSAP conformations highlighted in blue and red, respectively.

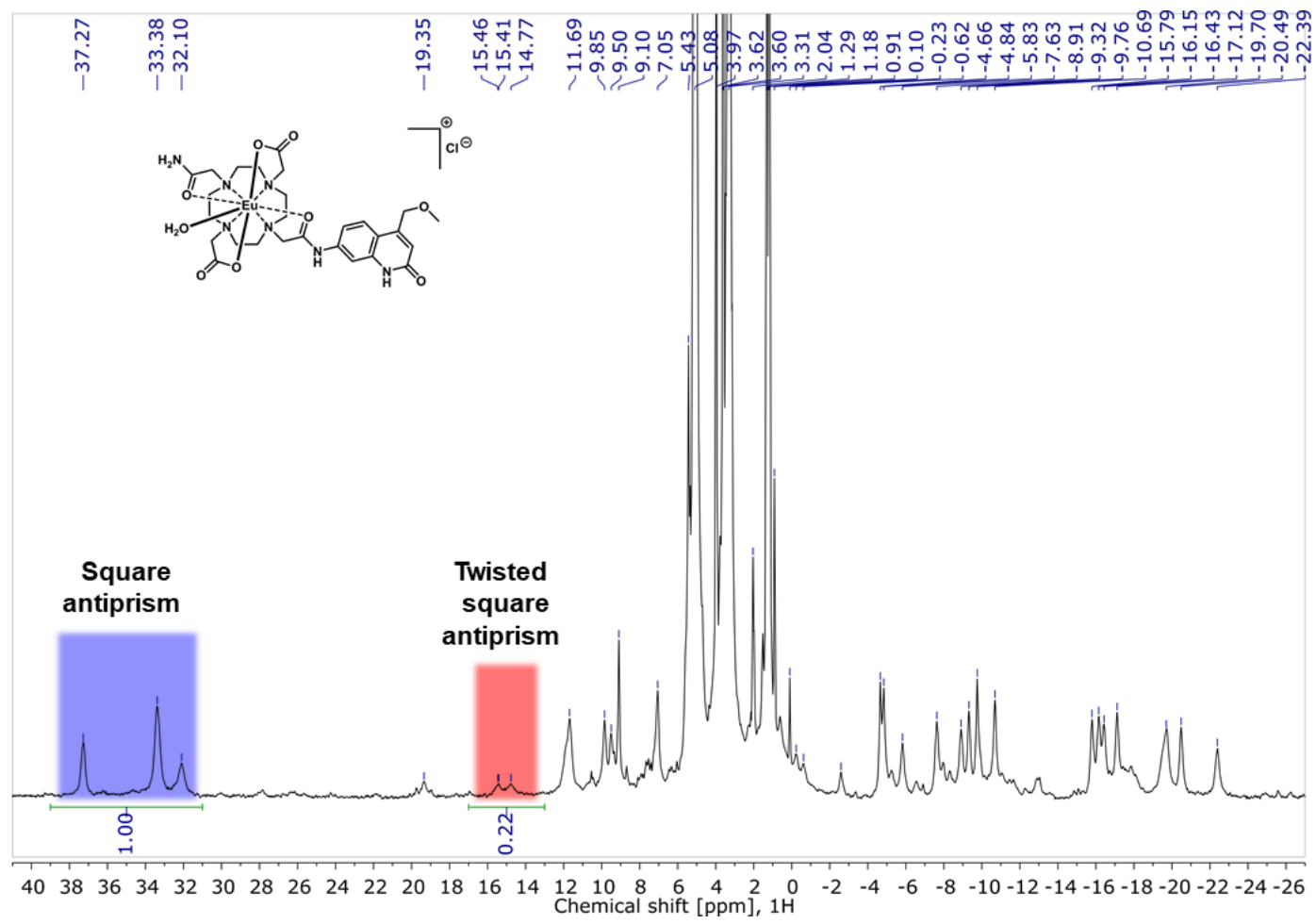

Figure S2. ${ }^{1} \mathrm{H}$ NMR spectrum of EuL2 ${ }^{\mathrm{MOM}}\left(400 \mathrm{MHz}, \mathrm{CD}_{3} \mathrm{OD}, 0{ }^{\circ} \mathrm{C}\right.$ ) with the regions corresponding to the SAP and TSAP conformations highlighted in blue and red, respectively. 


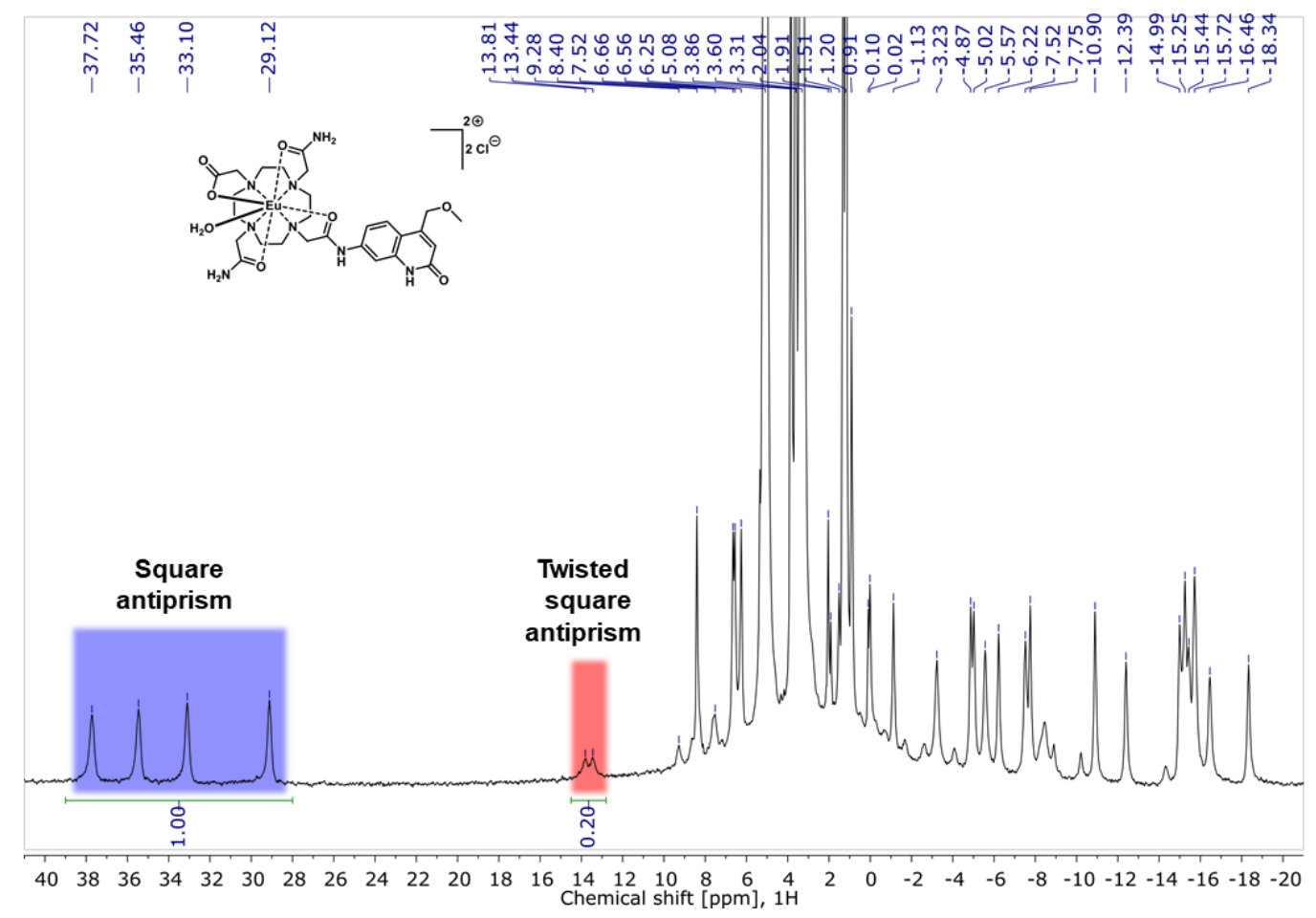

Figure S3. ${ }^{1} \mathrm{H}$ NMR spectrum of EuL3 ${ }^{\text {MOM }}\left(400 \mathrm{MHz}, \mathrm{CD}_{3} \mathrm{OD}, 0{ }^{\circ} \mathrm{C}\right.$ ) with the regions corresponding to the SAP and TSAP conformations highlighted in blue and red, respectively.

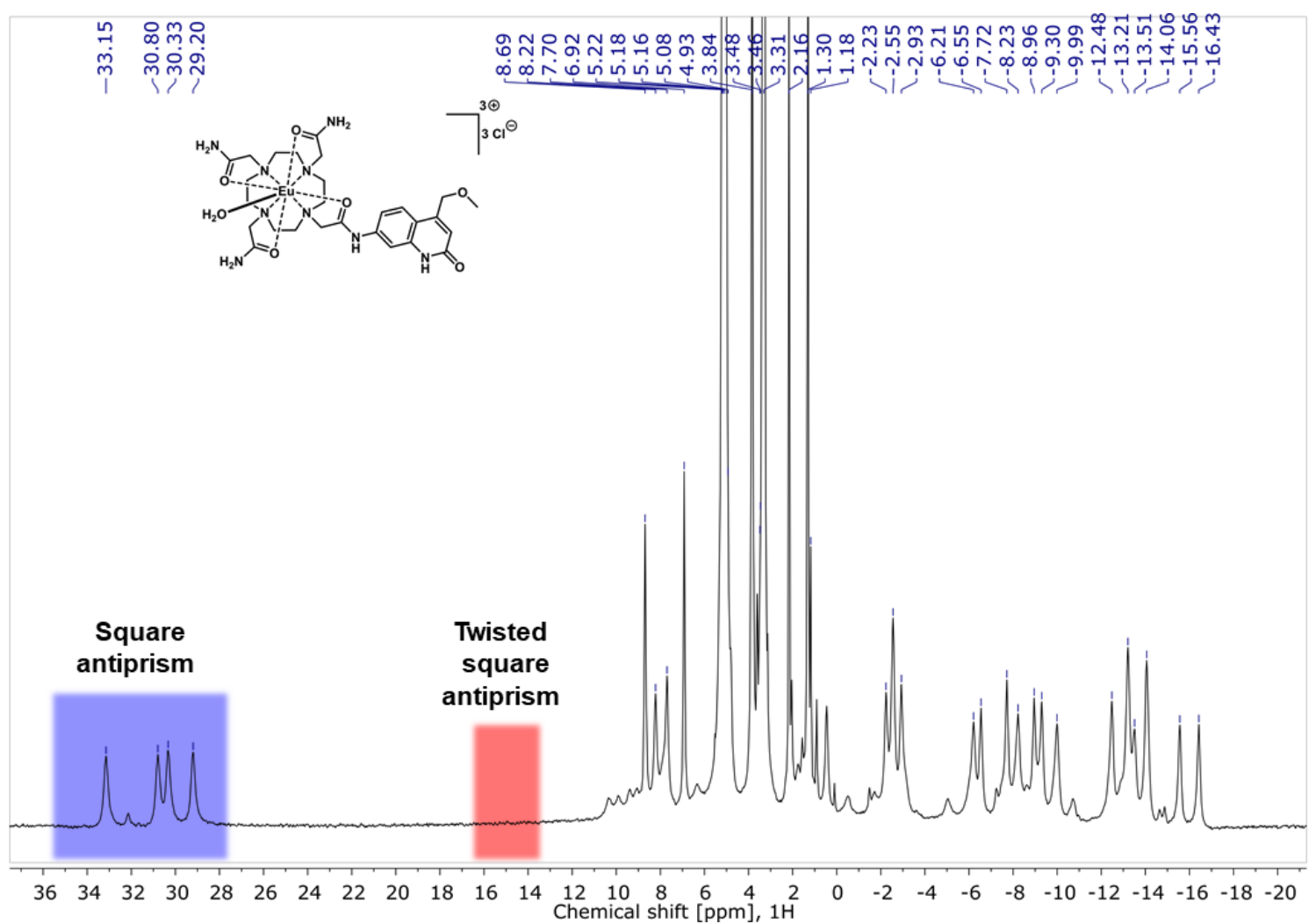

Figure S4. ${ }^{1} \mathrm{H}$ NMR spectrum of EuL4a ${ }^{\text {MOM }}\left(400 \mathrm{MHz}, \mathrm{CD}_{3} \mathrm{OD}, 0{ }^{\circ} \mathrm{C}\right)$ with the regions corresponding to the SAP and TSAP conformations highlighted in blue and red, respectively. 


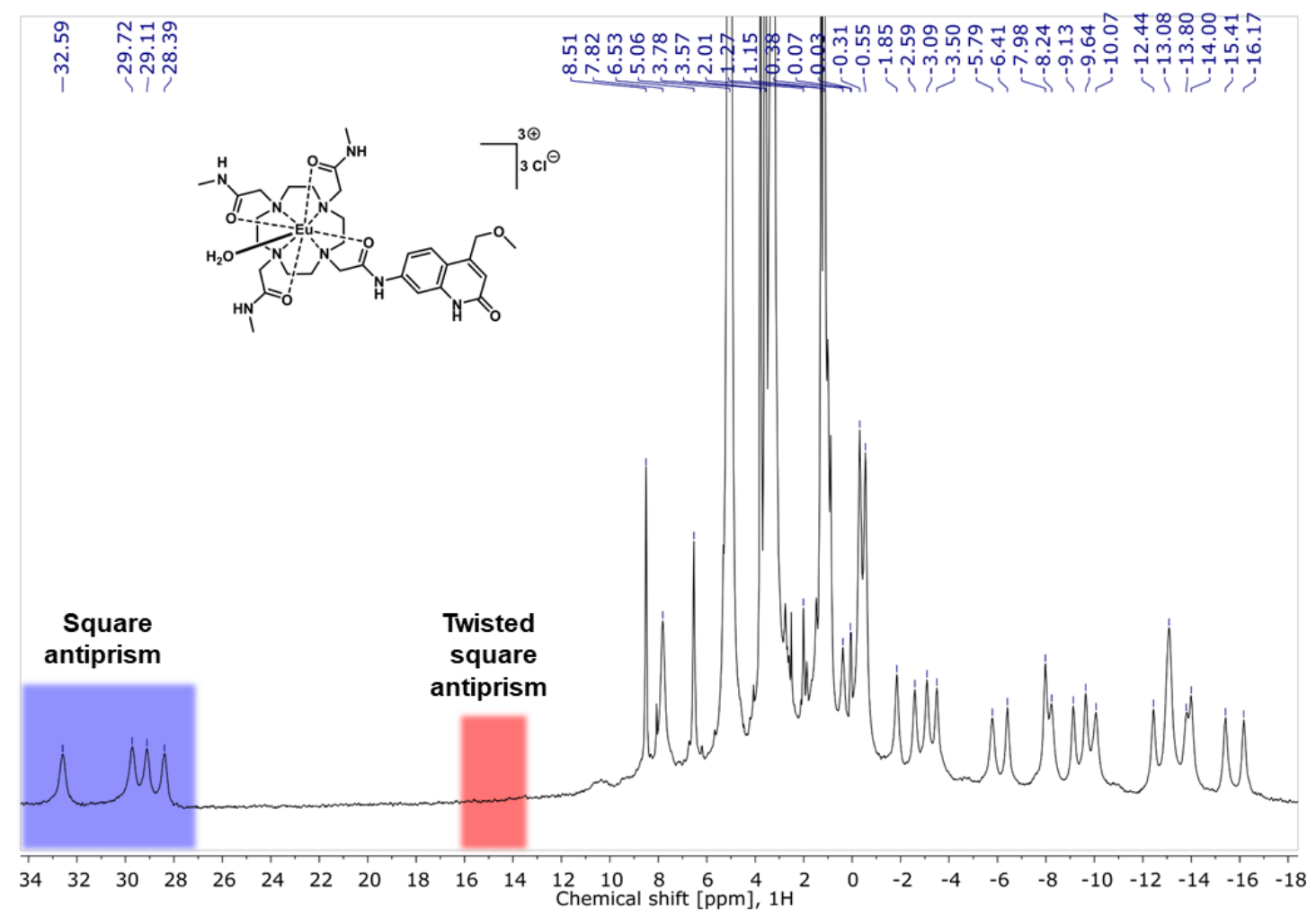

Figure S5. ${ }^{1} \mathrm{H}$ NMR spectrum of EuL4b ${ }^{\mathrm{MOM}}\left(400 \mathrm{MHz}, \mathrm{CD}_{3} \mathrm{OD}, 0{ }^{\circ} \mathrm{C}\right.$ ) with the regions corresponding to the SAP and TSAP conformations highlighted in blue and red, respectively.

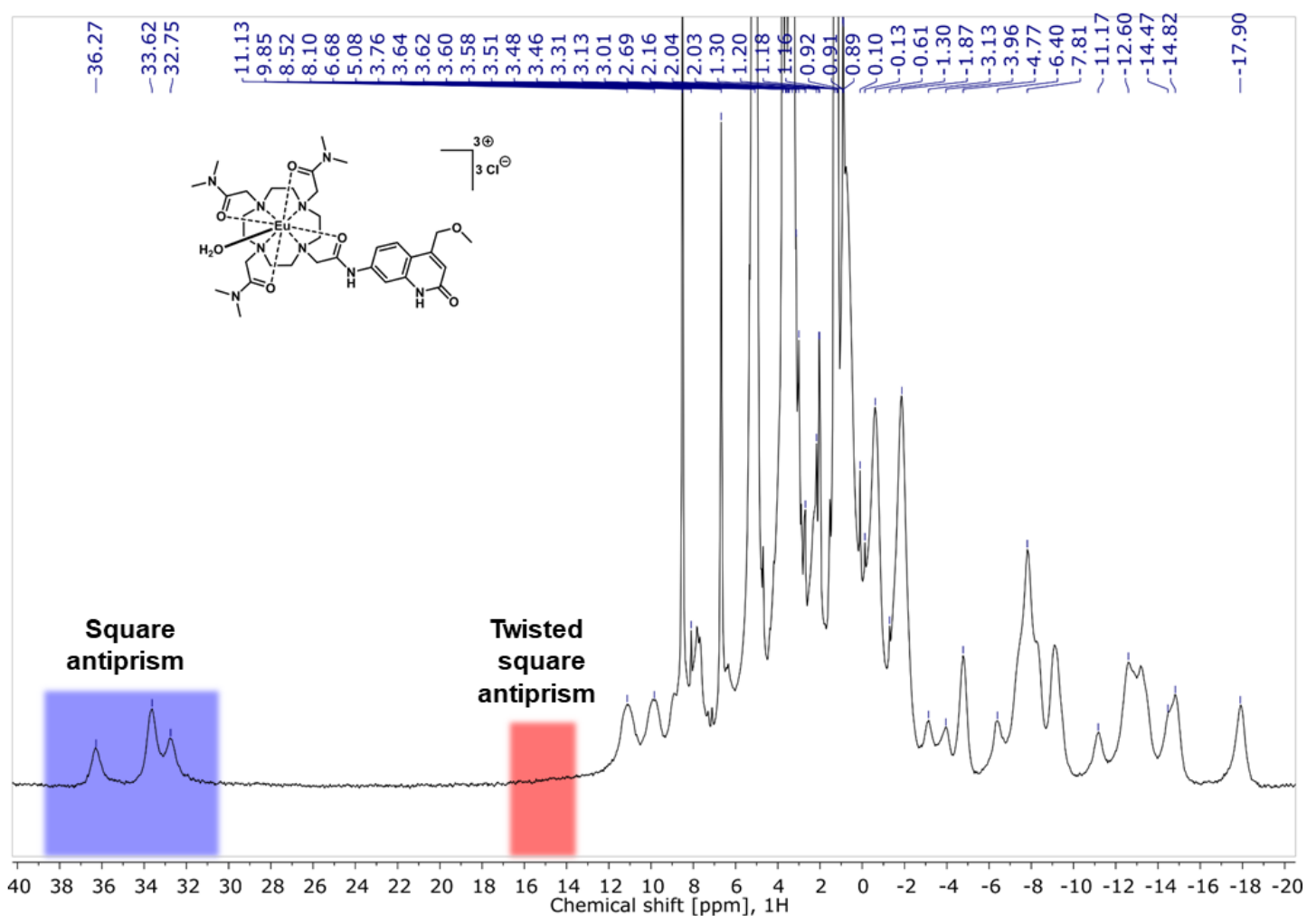

Figure S6. ${ }^{1} \mathrm{H}$ NMR spectrum of EuL4c ${ }^{\text {MOM }}$ (400 $\mathrm{MHz}, \mathrm{CD}_{3} \mathrm{OD}, 0{ }^{\circ} \mathrm{C}$ ) with the regions corresponding to the SAP and TSAP conformations highlighted in blue and red, respectively. 


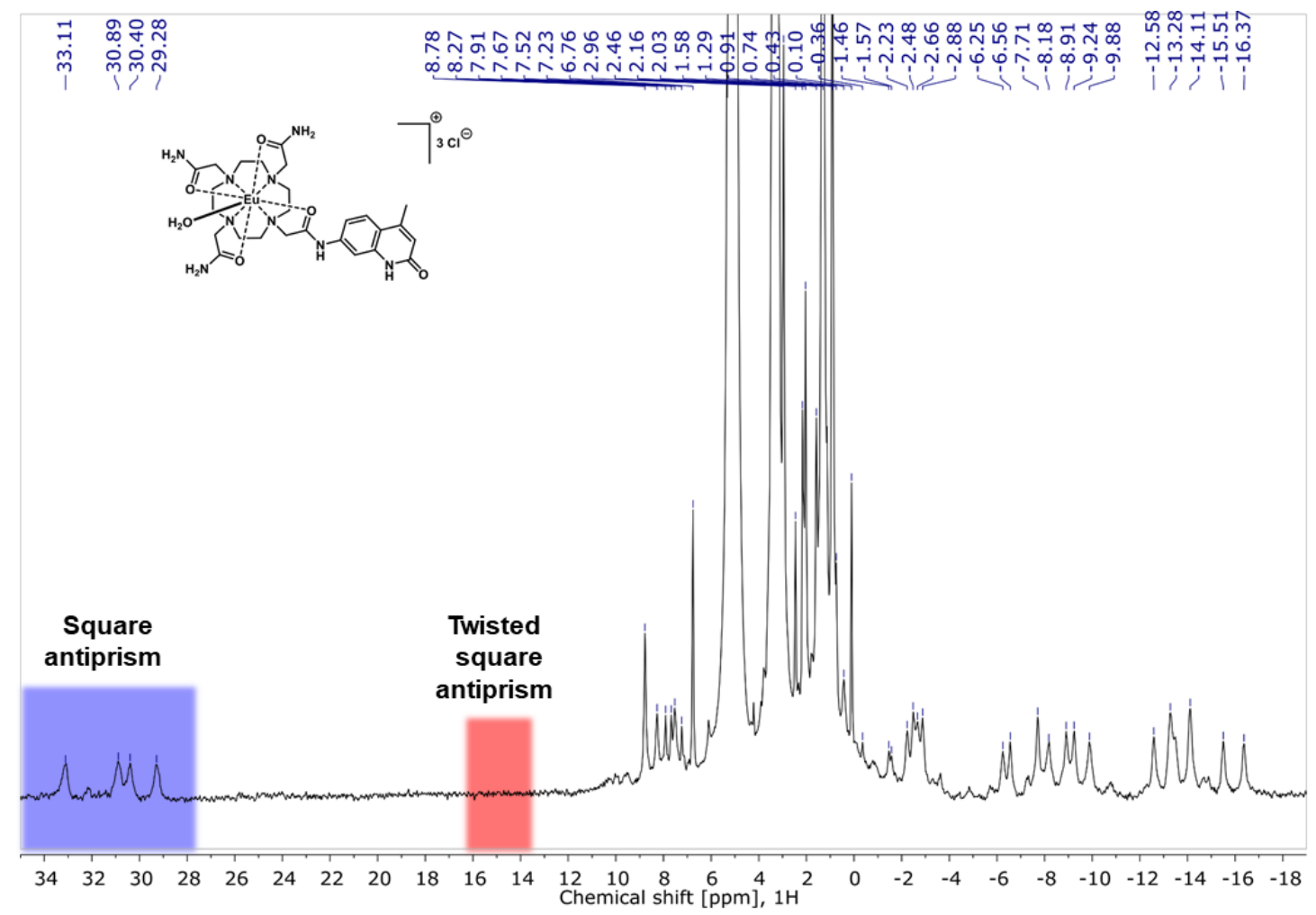

Figure S7. ${ }^{1} \mathrm{H}$ NMR spectrum of EuL4a $\mathbf{a}^{\mathrm{Me}}\left(400 \mathrm{MHz}, \mathrm{CD}_{3} \mathrm{OD}, 0{ }^{\circ} \mathrm{C}\right.$ ) with the regions corresponding to the SAP and TSAP conformations highlighted in blue and red, respectively.

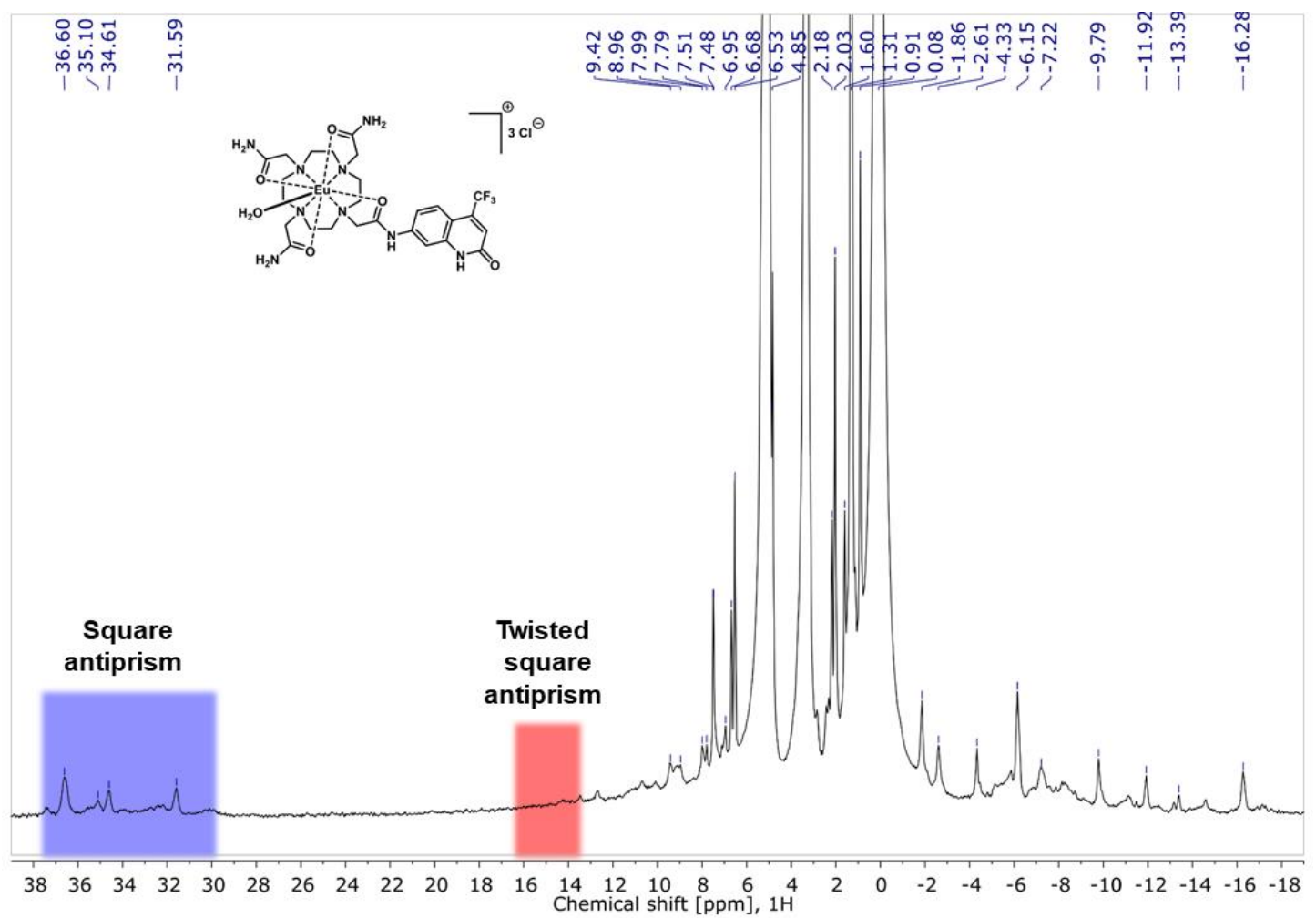

Figure S8. ${ }^{1} \mathrm{H}$ NMR spectrum of EuL4a ${ }^{\mathrm{CF} 3}\left(400 \mathrm{MHz}, \mathrm{CD}_{3} \mathrm{OD}, 0{ }^{\circ} \mathrm{C}\right)$ with the regions corresponding to the SAP and TSAP conformations highlighted in blue and red, respectively. 


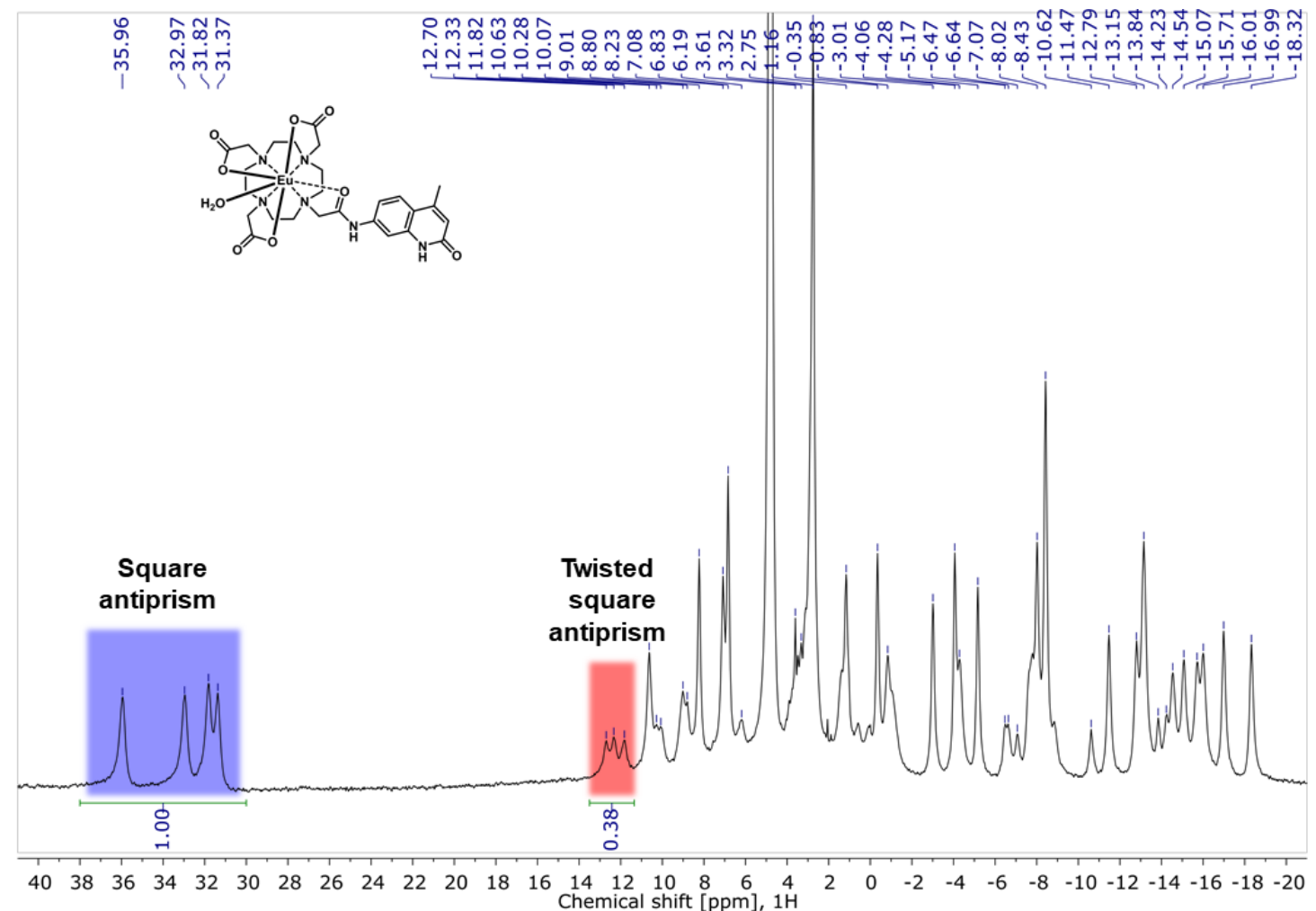

Figure S9. ${ }^{1} \mathrm{H}$ NMR spectrum of $\mathbf{E u L 1}^{\mathrm{Me}}\left(400 \mathrm{MHz}, \mathrm{D}_{2} \mathrm{O}, 10{ }^{\circ} \mathrm{C}\right)$ with the regions corresponding to the SAP and TSAP conformations highlighted in blue and red, respectively.

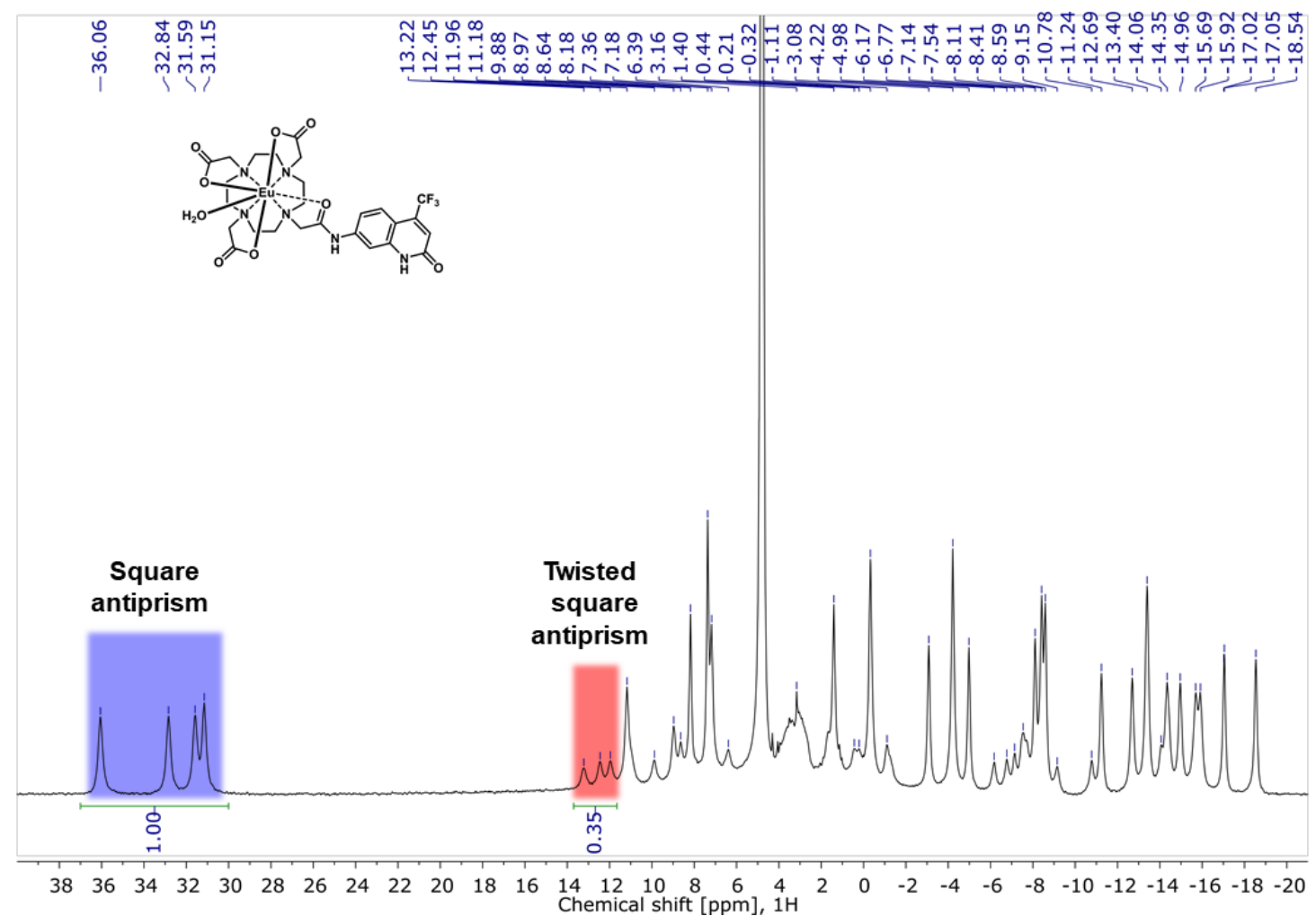

Figure S10. ${ }^{1} \mathrm{H}$ NMR spectrum of EuL1 ${ }^{\mathbf{C F} 3}\left(400 \mathrm{MHz}, \mathrm{D}_{2} \mathrm{O}, 10{ }^{\circ} \mathrm{C}\right.$ ) with the regions corresponding to the SAP and TSAP conformations highlighted in blue and red, respectively. 


\section{X-ray diffraction data}
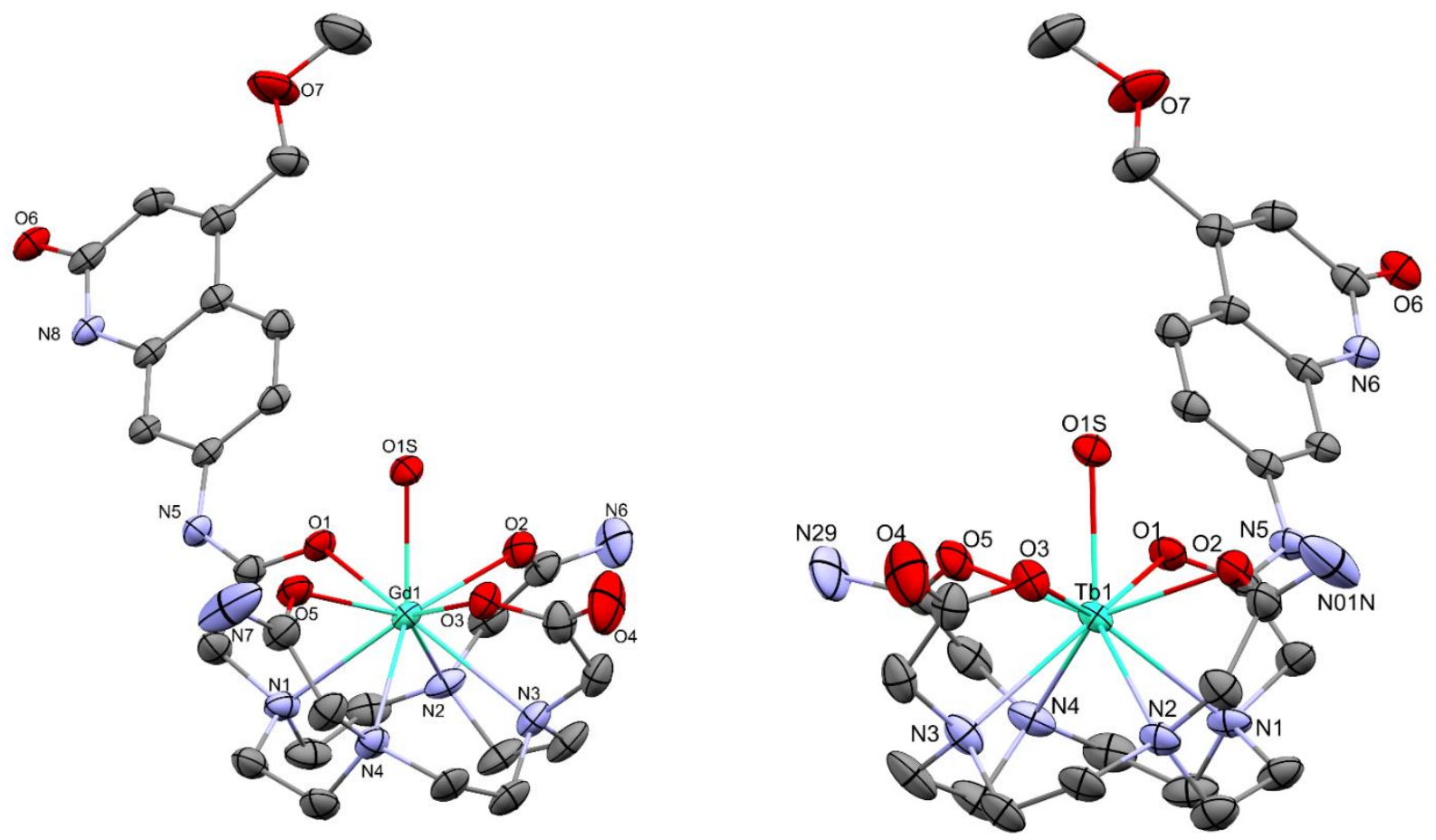

Figure S11. Solid-state structures of GdL3 $3^{\text {MOM }}$ (left) and TbL3 $3^{\text {MOM }}$ (right). H atoms, noncoordinating $\mathrm{Cl}^{-}$counterions and water molecules are omitted for clarity. Ellipsoids displayed at $50 \%$ probability.
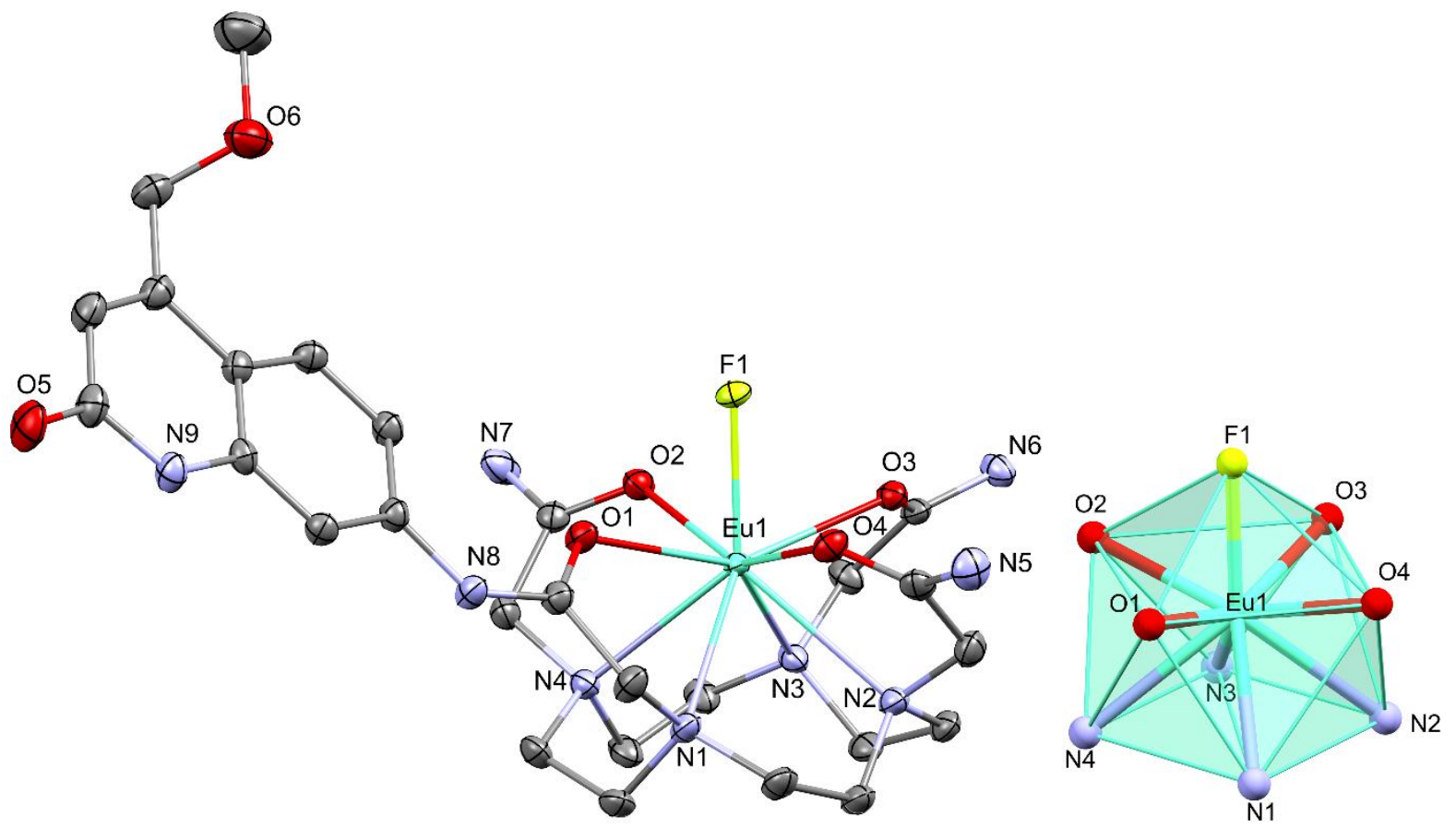

Figure S12. Solid-state structure of EuL4a ${ }^{\text {MOM}}-\mathbf{F}$ (left) and coordination environment of the metal centre (right). $\mathrm{H}$ atoms, non-coordinating $\mathrm{Cl}^{-}$counterions and water molecules are omitted for clarity. Ellipsoids displayed at $50 \%$ probability. 


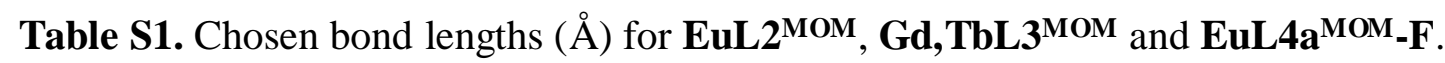

\begin{tabular}{|c|c|c|c|c|c|}
\hline \multirow[t]{2}{*}{ Parameter } & \multicolumn{2}{|c|}{ EuL2MOM } & \multirow[t]{2}{*}{ GdL3 $^{\text {MOM }}$} & \multirow[t]{2}{*}{ TbL3 ${ }^{\text {MOM }}$} & \multirow[t]{2}{*}{$\mathbf{E u L 4 a}^{\mathrm{MOM}}-\mathbf{F}$} \\
\hline & Eu1 & Eu2 & & & \\
\hline $\mathbf{X}=$ & $\mathrm{H}_{2} \mathrm{O}$ & $\mu-\mathrm{AcO}^{-}$ & $\mathrm{H}_{2} \mathrm{O}$ & $\mathrm{H}_{2} \mathrm{O}$ & $\mathbf{F}^{-}$ \\
\hline Ln-Oav & $2.367(3)$ & $2.380(3)$ & $2.360(3)$ & $2.348(3)$ & $2.406(2)$ \\
\hline Ln-O1 & $2.479(2)$ & $2.429(2)$ & $2.437(3)$ & $2.427(3)$ & $2.446(2)$ \\
\hline $\mathbf{L n}-\mathbf{N}_{\mathrm{av}}$ & $2.645(3)$ & $2.672(3)$ & $2.650(4)$ & $2.643(4)$ & $2.702(2)$ \\
\hline Ln-X & $2.427(3)$ & $2.390(3)$ & $2.399(3)$ & $2.398(3)$ & $2.225(2)$ \\
\hline $\mathrm{Ln}-\mathrm{O}_{4 \mathrm{PL}}$ & 0.734 & 0.701 & 0.733 & 0.739 & 0.642 \\
\hline Ln-N4PL & 1.604 & 1.648 & 1.611 & 1.602 & 1.695 \\
\hline
\end{tabular}


Table S2. Torsion angles NCCN and NCCO $\left(^{\circ}\right)$ for $\mathbf{E u L 2} 2^{\text {MOM }}, \mathbf{L n L} 3^{\text {MOM }}(\operatorname{Ln}=\mathrm{Gd}, \mathrm{Tb})$ and EuL4a $\mathbf{a}^{\text {MOM.F. }}$

\begin{tabular}{|c|c|c|c|c|c|c|c|c|}
\hline \multirow[t]{2}{*}{ Parameter } & \multicolumn{2}{|c|}{ EuL2 ${ }^{\text {MOM }}$} & \multicolumn{2}{|c|}{ GdL3 $^{\text {MOM }}$} & \multicolumn{2}{|c|}{ TbL3 ${ }^{\text {MOM }}$} & \multicolumn{2}{|c|}{ EuL4a $^{\text {MOM_F }}$} \\
\hline & Eu1 & Eu2 & Gd1 & Gd2 & Tb1 & Tb2 & Eu1 & Eu2 \\
\hline $\mathbf{X}=$ & $\mathrm{H}_{2} \mathrm{O}$ & $\mu-\mathrm{AcO}^{-}$ & $\mathrm{H}_{2} \mathrm{O}$ & $\mathrm{H}_{2} \mathrm{O}$ & $\mathrm{H}_{2} \mathrm{O}$ & $\mathrm{H}_{2} \mathrm{O}$ & $\mathbf{F}^{-}$ & $\mathbf{F}^{-}$ \\
\hline $\mathrm{N}_{1} \mathrm{C}_{1} \mathrm{C}_{2} \mathrm{~N}_{2}$ & -62.82 & $60.28^{a}$ & 56.74 & $-61.64^{a}$ & -59.93 & $61.28^{a}$ & 58.89 & $-58.37^{a}$ \\
\hline $\mathrm{N}_{2} \mathrm{C}_{5} \mathrm{C}_{6} \mathrm{~N}_{3}$ & -57.19 & $64.18^{b}$ & 57.72 & $-56.66^{b}$ & -56.64 & $57.09^{b}$ & 57.44 & $-59.51^{b}$ \\
\hline $\mathrm{N}_{3} \mathrm{C}_{9} \mathrm{C}_{10} \mathrm{~N}_{4}$ & -60.90 & $58.85^{\mathrm{c}}$ & 58.22 & $-58.79^{c}$ & -58.33 & $60.15^{c}$ & 59.98 & $-59.00^{\mathrm{c}}$ \\
\hline $\mathrm{N}_{4} \mathrm{C}_{13} \mathrm{C}_{14} \mathrm{~N}_{1}$ & -54.00 & $58.29^{d}$ & 61.05 & $-59.02^{d}$ & -57.12 & $59.57^{d}$ & 58.10 & $-59.39^{d}$ \\
\hline $\begin{array}{l}\text { Mean NCCN } \\
\text { torsion angle }\end{array}$ & -58.73 & 60.40 & 58.43 & -59.03 & -58.01 & 59.52 & 58.60 & -59.07 \\
\hline $\mathrm{N}_{1} \mathrm{C}_{15} \mathrm{C}_{16} \mathrm{O}_{1}$ & 36.04 & $-24.50^{\mathrm{e}}$ & -29.60 & $21.81^{\mathrm{e}}$ & 29.14 & $-22.14^{\mathrm{e}}$ & -36.52 & $32.33^{\mathrm{e}}$ \\
\hline $\mathrm{N}_{2} \mathrm{C}_{3} \mathrm{C}_{4} \mathrm{O}_{2}$ & 5.96 & $-8.01^{\mathrm{f}}$ & -37.61 & $19.95^{\mathrm{f}}$ & 13.47 & $-21.54^{f}$ & $-29.24 \mathrm{j}$ & $25.85^{f}$ \\
\hline $\mathrm{N}_{3} \mathrm{C}_{7} \mathrm{C}_{8} \mathrm{O}_{4}$ & 26.89 & $-40.05^{\mathrm{g}}$ & $-20.77 \mathrm{i}$ & $20.55^{\mathrm{g}}$ & $21.91^{\mathrm{i}}$ & $-21.24 \mathrm{~g}$ & $-31.83^{i}$ & $23.01^{\mathrm{g}}$ \\
\hline $\mathrm{N}_{4} \mathrm{C}_{11} \mathrm{C}_{12} \mathrm{O}_{5}$ & 28.40 & $-24.11^{\mathrm{h}}$ & -13.54 & $33.30^{\mathrm{h}}$ & 36.72 & $-33.60^{h}$ & $-28.24^{k}$ & $36.36^{h}$ \\
\hline $\begin{array}{l}\text { Mean NCCO } \\
\text { torsion angle }\end{array}$ & 24.32 & -24.17 & -20.77 & 23.90 & 25.31 & -24.63 & -31.46 & 29.39 \\
\hline
\end{tabular}

${ }^{a}$ The torsion angle of $\mathrm{N}_{\mathrm{w}} \mathrm{C}_{28} \mathrm{C}_{29} \mathrm{~N}_{\mathrm{x}}(\mathrm{w}=7,8,9,10 ; \mathrm{x}=8,9,10,11){ }^{\mathrm{b}}$ The torsion angle of $\mathrm{N}_{\mathrm{x}} \mathrm{C}_{32} \mathrm{C}_{33} \mathrm{~N}_{\mathrm{y}}(\mathrm{y}=9,10,11,12){ }^{\mathrm{c}}$ The torsion angle of $\mathrm{N}_{\mathrm{y}} \mathrm{C}_{36} \mathrm{C}_{37} \mathrm{~N}_{\mathrm{z}}(\mathrm{z}=10,11,12,13)$. ${ }^{\mathrm{d}}$ The torsion angle of $\mathrm{N}_{\mathrm{z}} \mathrm{C}_{40} \mathrm{C}_{41} \mathrm{~N}_{\mathrm{w}}$. ${ }^{\mathrm{e}}$ The torsion angle of $\mathrm{N}_{\mathrm{w}} \mathrm{C}_{42} \mathrm{C}_{43} \mathrm{O}_{\mathrm{x}}$. ${ }^{\mathrm{f}} \mathrm{The}^{\mathrm{C}}$ torsion angle of $\mathrm{N}_{\mathrm{x}} \mathrm{C}_{30} \mathrm{C}_{31} \mathrm{O}_{\mathrm{y}}$. ${ }^{\mathrm{g}}$ The torsion angle of $\mathrm{N}_{\mathrm{y}} \mathrm{C}_{34} \mathrm{C}_{35} \mathrm{O}_{\mathrm{z} .}{ }^{\mathrm{h}}$ The torsion angle of $\mathrm{N}_{\mathrm{z}} \mathrm{C}_{38} \mathrm{C}_{39} \mathrm{O}_{\mathrm{w} .}{ }^{\mathrm{i}}$ The torsion angle of $\mathrm{N}_{3} \mathrm{C}_{7} \mathrm{C}_{8} \mathrm{O}_{3}$. ${ }^{\mathrm{j}} \mathrm{The}$ torsion angle of $\mathrm{N}_{2} \mathrm{C}_{3} \mathrm{C}_{4} \mathrm{O}_{4}$. ${ }^{\mathrm{k}}$ The torsion angle of $\mathrm{N}_{4} \mathrm{C}_{11} \mathrm{C}_{12} \mathrm{O}_{2}$. 
Table S3. Crystal data and structure refinement for EuL2 ${ }^{\text {MOM }}$, Gd, TbL3 ${ }^{\text {MOM }}$ and EuL4a ${ }^{\text {MOM }}$ F.

\begin{tabular}{|c|c|c|c|c|}
\hline Compound & EuL2 $^{\text {MOM }}$ & GdL3 $^{\text {MOM }}$ & TbL3 $^{\text {MOM }}$ & EuL4a $^{\text {MOM}_{-}}$F \\
\hline $\begin{array}{l}\text { Chemical } \\
\text { formula }\end{array}$ & $\begin{array}{c}\mathrm{C}_{54} \mathrm{H}_{76} \mathrm{Eu}_{2} \mathrm{~N}_{14} \mathrm{O}_{17} \\
\cdot 2(\mathrm{Cl}) \cdot 12.5\left(\mathrm{H}_{2} \mathrm{O}\right) \\
\cdot 3(\mathrm{HO}) \cdot 1.85(\mathrm{O}) \\
\cdot \mathrm{HO}_{0.65}\end{array}$ & $\begin{array}{c}2\left(\mathrm{C}_{27} \mathrm{H}_{39} \mathrm{GdN}_{8} \mathrm{O}_{8}\right) \\
\cdot 4(\mathrm{Cl}) \cdot 7(\mathrm{O})\end{array}$ & $\begin{array}{c}2\left(\mathrm{C}_{27} \mathrm{H}_{39} \mathrm{~N}_{8} \mathrm{O}_{8} \mathrm{~Tb}\right) \\
\cdot 4(\mathrm{Cl}) \cdot 6.5(\mathrm{O})\end{array}$ & $\begin{array}{c}2\left(\mathrm{C}_{27} \mathrm{H}_{41} \mathrm{EuFN}_{9} \mathrm{O}_{6}\right) \\
.4(\mathrm{Cl}) \cdot 2(\mathrm{HO}) \\
.6\left(\mathrm{H}_{2} \mathrm{O}\right) \cdot 8(\mathrm{O})\end{array}$ \\
\hline$M_{\mathrm{r}}$ & 1885.33 & 1775.62 & 1770.96 & 1929.2 \\
\hline \multirow{2}{*}{$\begin{array}{l}\text { Crystal system, } \\
\text { space group }\end{array}$} & Monoclinic & Monoclinic & Monoclinic & Triclinic \\
\hline & $P 21 / c$ & $\mathrm{C} 2 / \mathrm{c}$ & $C 2 / c$ & $P^{-} 1$ \\
\hline Temperature $(\mathbf{K})$ & 170 & 170 & 170 & 150 \\
\hline \multirow{3}{*}{$a, b, c(\AA)$} & $17.6443(6)$ & 21.004 (2) & $20.961(2)$ & $13.1182(5)$ \\
\hline & $34.154(1)$ & $19.732(2)$ & $19.691(2)$ & $17.8275(7)$ \\
\hline & $13.9203(5)$ & $43.598(4)$ & $43.577(4)$ & $19.2160(7)$ \\
\hline \multirow{3}{*}{$\alpha, \boldsymbol{\beta}, \gamma\left({ }^{\circ}\right)$} & - & - & - & $114.961(1)$ \\
\hline & $109.441(1)$ & $93.046(2)$ & $93.086(2)$ & $97.045(1)$ \\
\hline & - & - & - & $93.992(1)$ \\
\hline$V\left(\AA^{3}\right)$ & $7910.5(5)$ & $18044(3)$ & $17960(2)$ & $4006.5(3)$ \\
\hline$Z$ & 4 & 8 & 8 & 2 \\
\hline Radiation type & Mo K $\alpha$ & Mo K $\alpha$ & Mo Ka & Mo $K \alpha$ \\
\hline$\mu\left(\mathrm{mm}^{-1}\right)$ & 1.73 & 1.64 & 1.75 & 1.78 \\
\hline $\begin{array}{l}\text { Crystal size } \\
(\mathbf{m m})\end{array}$ & $0.6 \times 0.3 \times 0.2$ & $0.5 \times 0.2 \times 0.05$ & $0.6 \times 0.5 \times 0.1$ & $0.15 \times 0.13 \times 0.1$ \\
\hline Diffractometer & Bruker D8 APEX-II & Bruker D8 APEX-II & Bruker D8 APEX-II & Bruker D8 APEX-II \\
\hline $\begin{array}{l}\text { Absorption } \\
\text { correction }\end{array}$ & Multi-scan & Multi-scan & Multi-scan & Multi-scan \\
\hline$T_{\min }, T_{\max }$ & $0.653,0.746$ & $0.622,0.746$ & $0.658,0.746$ & $0.637,0.746$ \\
\hline \multirow{3}{*}{$\begin{array}{l}\text { No. of measured, } \\
\text { independent and } \\
\text { observed [I > } \\
2 s(I)] \text { reflections }\end{array}$} & 348248 & 105196 & 135458 & 221802 \\
\hline & 18928 & 27578 & 27498 & 24587 \\
\hline & 16830 & 19883 & 19507 & 20765 \\
\hline$R_{\mathrm{int}}$ & 0.059 & 0.065 & 0.074 & 0.045 \\
\hline$(\sin \theta / \lambda)_{\max }\left(\AA^{-1}\right)$ & 0.659 & 0.716 & 0.716 & 0.716 \\
\hline $\begin{array}{l}R\left[F^{2}>2 \sigma\left(F^{2}\right)\right], \\
w R\left(F^{2}\right), S\end{array}$ & $0.037,0.092,1.11$ & $0.054,0.161,1.04$ & $0.054,0.157,1.05$ & $0.030,0.082,1.05$ \\
\hline $\begin{array}{l}\text { No. of } \\
\text { parameters }\end{array}$ & 1130 & 932 & 938 & 1061 \\
\hline No. of restraints & 32 & 42 & 48 & 15 \\
\hline $\begin{array}{l}\text { H-atom } \\
\text { treatment }\end{array}$ & $\begin{array}{l}\text { Independent and } \\
\text { constrained }\end{array}$ & Constrained & Constrained & $\begin{array}{l}\text { Independent and } \\
\text { constrained }\end{array}$ \\
\hline $\begin{array}{l}\left.\Delta\rangle_{\max }, \Delta\right\rangle_{\min }\left(\mathrm{e} \AA^{-}\right. \\
\left.{ }^{-}\right)\end{array}$ & $1.90,-1.01$ & $2.55,-1.30$ & $3.00,-1.22$ & $1.99,-1,19$ \\
\hline CCDC No. & 1993035 & 1993033 & 1993034 & 1993036 \\
\hline
\end{tabular}


Selected Ln-O, Ln-N and Ln-X distances (where X denotes the ligand which caps the square antiprism) are displayed in Table S4, and follow the convention outlined in Figure S13. All the crystallographically characterized complexes comprise of two lanthanide complexes in the asymmetric unit. Ligand atoms numbered 1-4 correspond to those coordinated to Ln1 while 5-8 correspond to $\operatorname{Ln} 2$.

Table S4. Selected bond lengths ( $⿱$ A) for EuL2 ${ }^{\text {MOM }}$, Gd,TbL3 $3^{\text {MOM }}$ and EuL4a $\mathbf{a}^{\text {MOM}}-$ F. The X ligand denotes the ligand which occupies the capping position of the capped square antiprism.

\begin{tabular}{|c|c|c|c|c|}
\hline Parameter & EuL2 ${ }^{\mathrm{MOM}}$ & $\mathrm{GdL3}^{\mathrm{MOM}}$ & TbL3 $3^{\text {MOM }}$ & EuL4a $^{\text {MOM}_{-F}}$ \\
\hline Ln1-01 & $2.479(2)$ & $2.435(3)$ & $2.423(4)$ & $2.472(2)$ \\
\hline Ln1-O2 & $2.347(3)$ & $2.364(3)$ & $2.356(3)$ & $2.404(2)$ \\
\hline Ln1-O3 & $2.404(2)$ & $2.357(3)$ & $2.347(3)$ & $2.405(2)$ \\
\hline Ln1-04 & $2.350(2)$ & $2.362(3)$ & $2.338(4)$ & $2.399(2)$ \\
\hline Ln2-O5 & $2.429(2)$ & $2.393(2)$ & $2.376(3)$ & $2.421(2)$ \\
\hline Ln2-O6 & $2.336(2)$ & $2.367(3)$ & $2.355(4)$ & $2.414(2)$ \\
\hline Ln2-O7 & $2.426(3)$ & $2.343(3)$ & $2.329(3)$ & $2.404(2)$ \\
\hline Ln2-08 & $2.381(2)$ & $2.376(3)$ & $2.364(3)$ & $2.407(2)$ \\
\hline Ln1-N1 & $2.607(2)$ & $2.664(4)$ & $2.649(4)$ & $2.706(2)$ \\
\hline Ln1-N2 & $2.673(3)$ & $2.639(4)$ & $2.645(4)$ & $2.699(2)$ \\
\hline Ln1-N3 & $2.674(3)$ & $2.649(4)$ & $2.646(4)$ & $2.688(2)$ \\
\hline Ln1-N4 & $2.629(3)$ & $2.650(4)$ & $2.634(4)$ & $2.715(2)$ \\
\hline Ln2-N5 & $2.680(3)$ & $2.664(5)$ & $2.657(5)$ & $2.720(2)$ \\
\hline Ln2-N6 & $2.679(3)$ & $2.646(4)$ & $2.642(4)$ & $2.682(2)$ \\
\hline Ln2-N7 & $2.659(3)$ & $2.619(4)$ & $2.611(4)$ & $2.725(2)$ \\
\hline Ln2-N8 & $2.675(2)$ & $2.634(5)$ & $2.634(4)$ & $2.678(2)$ \\
\hline Ln1-X1 & $2.427(2)$ & $2.398(3)$ & $2.395(3)$ & $2.218(1)$ \\
\hline Ln2-X2 & 2.391(2) & 2.404(3) & $2.400(4)$ & $2.232(2)$ \\
\hline $\mathrm{X} 1=$ & $\mathrm{H}_{2} \mathrm{O}$ & $\mathrm{H}_{2} \mathrm{O}$ & $\mathrm{H}_{2} \mathrm{O}$ & $\mathrm{F}^{-}$ \\
\hline $\mathrm{X} 2=$ & $\mathrm{AcO}^{-}$ & $\mathrm{H}_{2} \mathrm{O}$ & $\mathrm{H}_{2} \mathrm{O}$ & $\mathrm{F}^{-}$ \\
\hline
\end{tabular}

Figure S13. Numbering convention for Table S4. 


\section{Cyclic voltammograms}

Table S5. $\mathrm{pH}$ values of the solutions used in cyclic voltammetry.

\begin{tabular}{llll}
\hline Compound & Replicate1 & Replicate2 & Replicate3 \\
\hline EuCl3 & 6.51 & - & 6.67 \\
EuL1 $^{\text {MOM }}$ & 6.26 & 6.49 & 6.46 \\
EuL2 $^{\text {MOM }}$ & 6.68 & 6.47 & 6.47 \\
EuL3 $^{\text {MOM }}$ & 6.73 & 6.50 & 6.57 \\
EuL4a $^{\text {MOM }}$ & 6.52 & 6.70 & 6.65 \\
EuL4b $^{\text {MOM }}$ & 6.49 & 6.66 & 6.41 \\
EuL4c $^{\text {MOM }}$ & 6.55 & 6.63 & 6.58 \\
EuL1 $^{\text {Me }}$ & 6.53 & 6.61 & 6.62 \\
EuL4a $^{\text {Me }}$ & - & 6.65 & 6.52 \\
EuL1 $^{\text {CF3 }}$ & 6.50 & 6.66 & 6.69 \\
EuL4a $^{\text {CF3 }}$ & - & 6.48 & 6.50 \\
\hline
\end{tabular}

Table S6. Cyclic voltammetry of Eu(III) complexes. ${ }^{[a]}$

\begin{tabular}{lllll}
\hline Compound & $\mathbf{E}_{\mathbf{p a}}{ }^{[\mathbf{a}]}$ & $\mathbf{E}_{\mathbf{p c}}{ }^{[\mathbf{a}]}$ & $\mathbf{E}_{\mathbf{1} / 2}{ }^{[\mathbf{a}]}$ & $\boldsymbol{\Delta E}^{[\mathbf{a}]}$ \\
\hline EuCl$^{[\mathbf{b}]}$ & $-220 \pm 50$ & $-543 \pm 58$ & $-382 \pm 4$ & 323 \\
\hline EuL1 $^{\text {Me [c] }}$ & $-773 \pm 8$ & $-894 \pm 5$ & $-833 \pm 2$ & 121 \\
EuL4a $^{\text {Me [b] }}$ & $-465 \pm 2$ & $-632 \pm 3$ & $-549 \pm 1$ & 177 \\
\hline EuL1 $^{\text {MOM [c] }}$ & $-771 \pm 9$ & $-908 \pm 34$ & $-839 \pm 13$ & 137 \\
EuL2 $^{\text {MOM [c] }}$ & $-695 \pm 2$ & $-800 \pm 11$ & $-747 \pm 5$ & 105 \\
EuL3 $^{\text {MOM [c] }}$ & $-593 \pm 6$ & $-684 \pm 5$ & $-638 \pm 3$ & 91 \\
EuL4a $^{\text {MOM [c] }}$ & $-472 \pm 17$ & $-643 \pm 12$ & $-554 \pm 8$ & 171 \\
EuL4b $^{\text {MOM [c] }}$ & $-531 \pm 9$ & $-676 \pm 15$ & $-603 \pm 4$ & 145 \\
EuL4c $^{\text {MOM [c] }}$ & $-571 \pm 38$ & $-771 \pm 37$ & $-671 \pm 4$ & 200 \\
\hline EuL1 $^{\text {CF3 [c] }}$ & $-770 \pm 8$ & $-894 \pm 10$ & $-832 \pm 4$ & 124 \\
EuL4a $^{\text {CF3 [b] }}$ & $-436 \pm 29$ & $-650 \pm 37$ & $-543 \pm 4$ & 214 \\
\hline
\end{tabular}

$\mathrm{E}_{\mathrm{pa}}\left(\mathrm{E}_{\mathrm{pc}}\right)$ anodic (cathodic) peak potential, $\mathrm{E}_{1 / 2}$ half-wave potential, $\Delta \mathrm{E}$ peaks separation. ${ }^{\text {[a] }}$ Values are in $\mathrm{mV}$ vs. NHE. Measured in $\mathrm{H}_{2} \mathrm{O}(\mathrm{LiCl} 0.1 \mathrm{M}, \mathrm{pH}$ 6.4-6.7) with a sample concentration of $1 \mathrm{mM}$ at a glassy $\mathrm{C}$ electrode using a SCE reference electrode and a Pt wire counter electrode with a scan rate of $0.10 \mathrm{~V} / \mathrm{s}$. ${ }^{[\mathrm{b}]}$ Data represent mean \pm standard deviation for two independent measurements. ${ }^{[c]}$ Data represent mean \pm standard deviation for three independent measurements. 


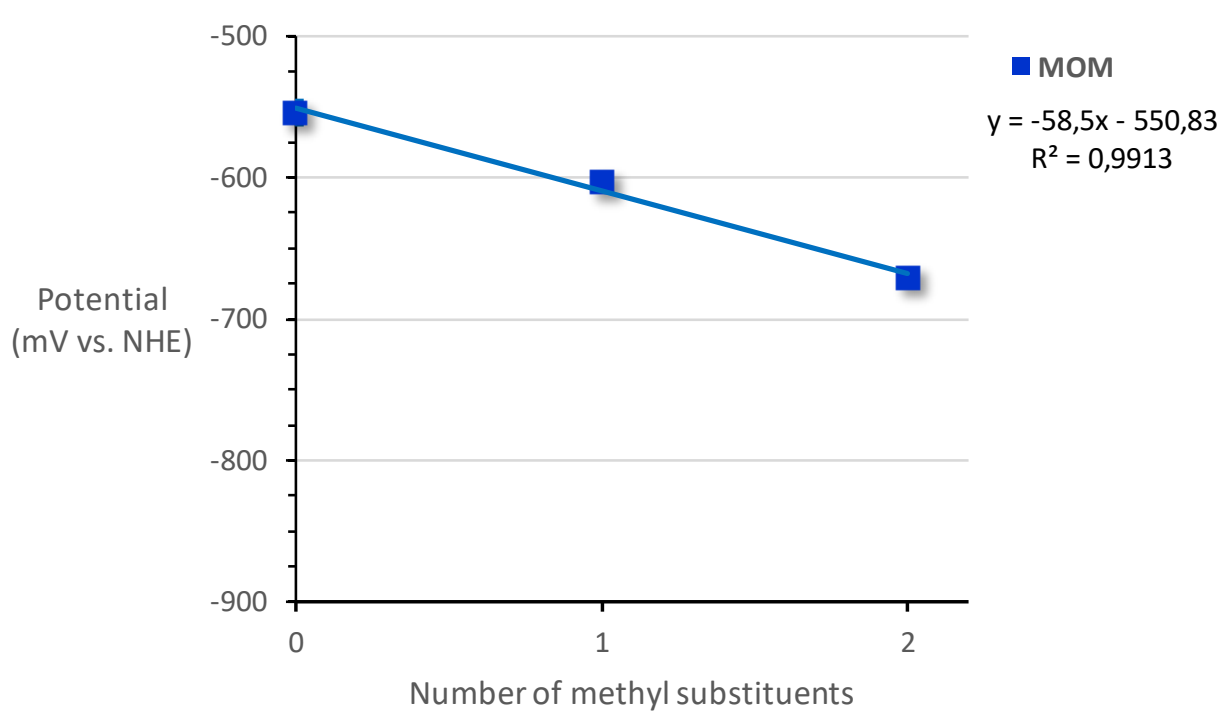

Figure S14. Apparent reduction potential vs. methyl substituent of EuL4a-c ${ }^{\text {MOM }}$ complexes. 

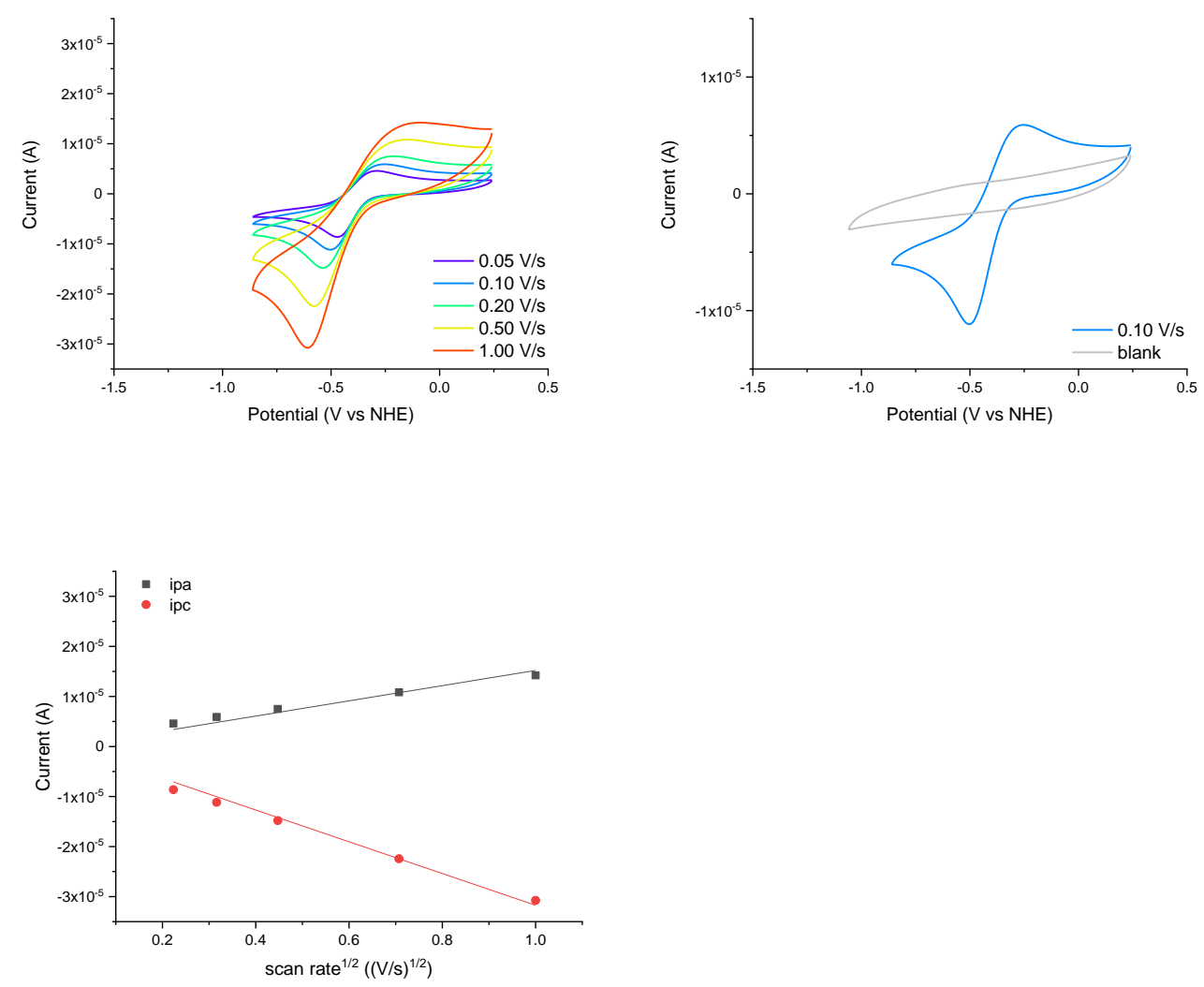

Figure S15. Cyclic voltammograms at various scan rate for $\mathrm{EuCl}_{3}$ and plot of peak current intensities vs. square root of scan rate.

Table S7. Values for linear fit of peak current intensities $v s$. square root of scan rate for $\mathrm{EuCl}_{3}$.

\begin{tabular}{ccc} 
Equation: $\mathbf{y}=\mathbf{a}^{*} \mathbf{x}$ & ipa & ipc \\
\hline Slope & $1.52 \cdot 10^{-5} \pm 7.42 \cdot 10^{-7}$ & $-3.17 \cdot 10^{-5} \pm 8.18 \cdot 10^{-7}$ \\
R-Square (COD) & 0.99056 & 0.99735 \\
\hline
\end{tabular}



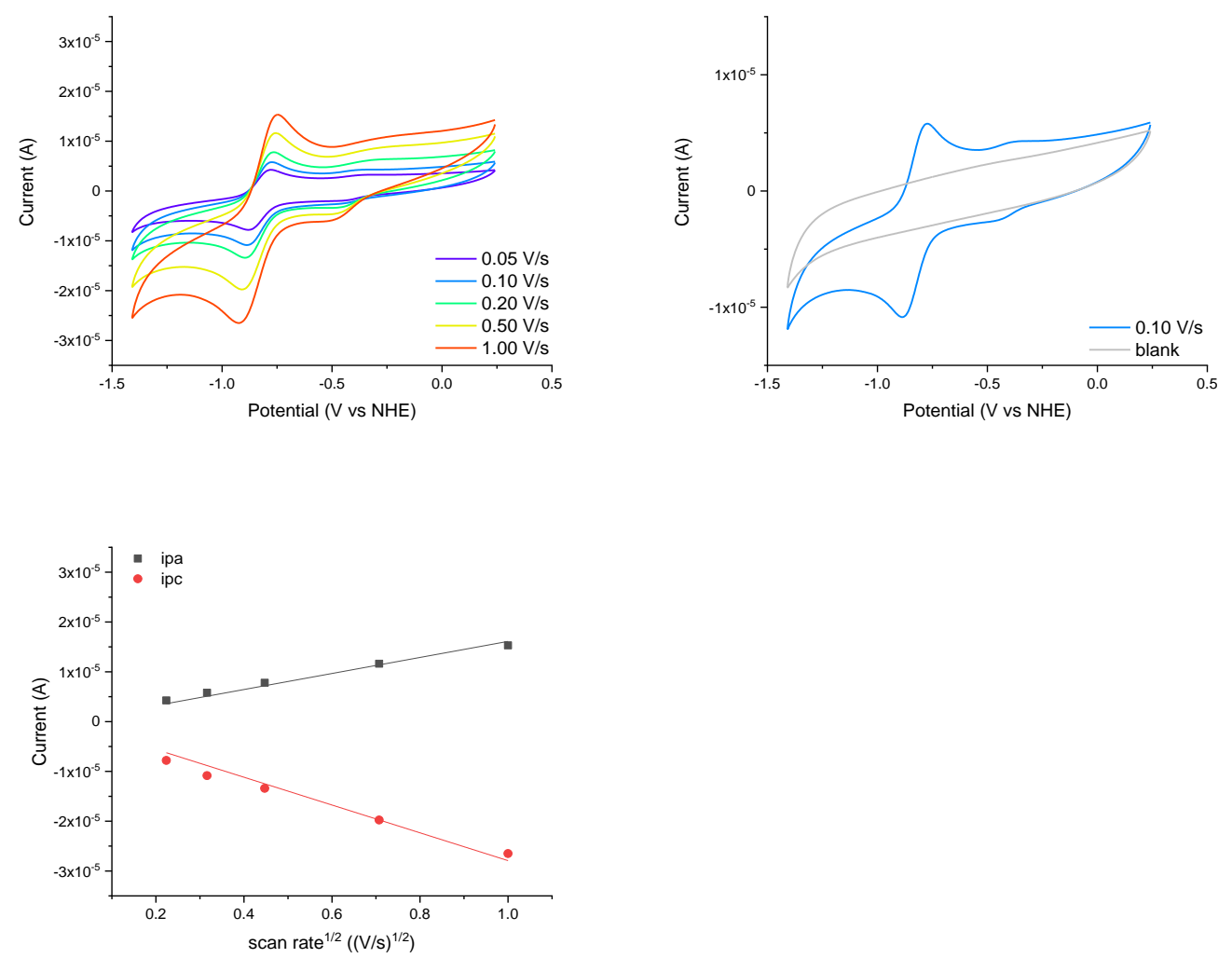

Figure S16. Cyclic voltammograms at various scan rate for EuL1 ${ }^{\text {MOM }}$ and plot of peak current intensities vs. square root of scan rate.

Table S8. Values for linear fit of peak current intensities $v s$. square root of scan rate for EuL1 ${ }^{\text {MOM. }}$

\begin{tabular}{lll} 
Equation: $\mathbf{y}=\mathbf{a}^{*} \mathbf{x}$ & ipa & ipc \\
\hline Slope & $1.61 \cdot 10^{-5} \pm 5.12 \cdot 10^{-7}$ & $-2.79 \cdot 10^{-5} \pm 1.12 \cdot 10^{-6}$ \\
R-Square (COD) & 0.99598 & 0.99356 \\
\hline
\end{tabular}



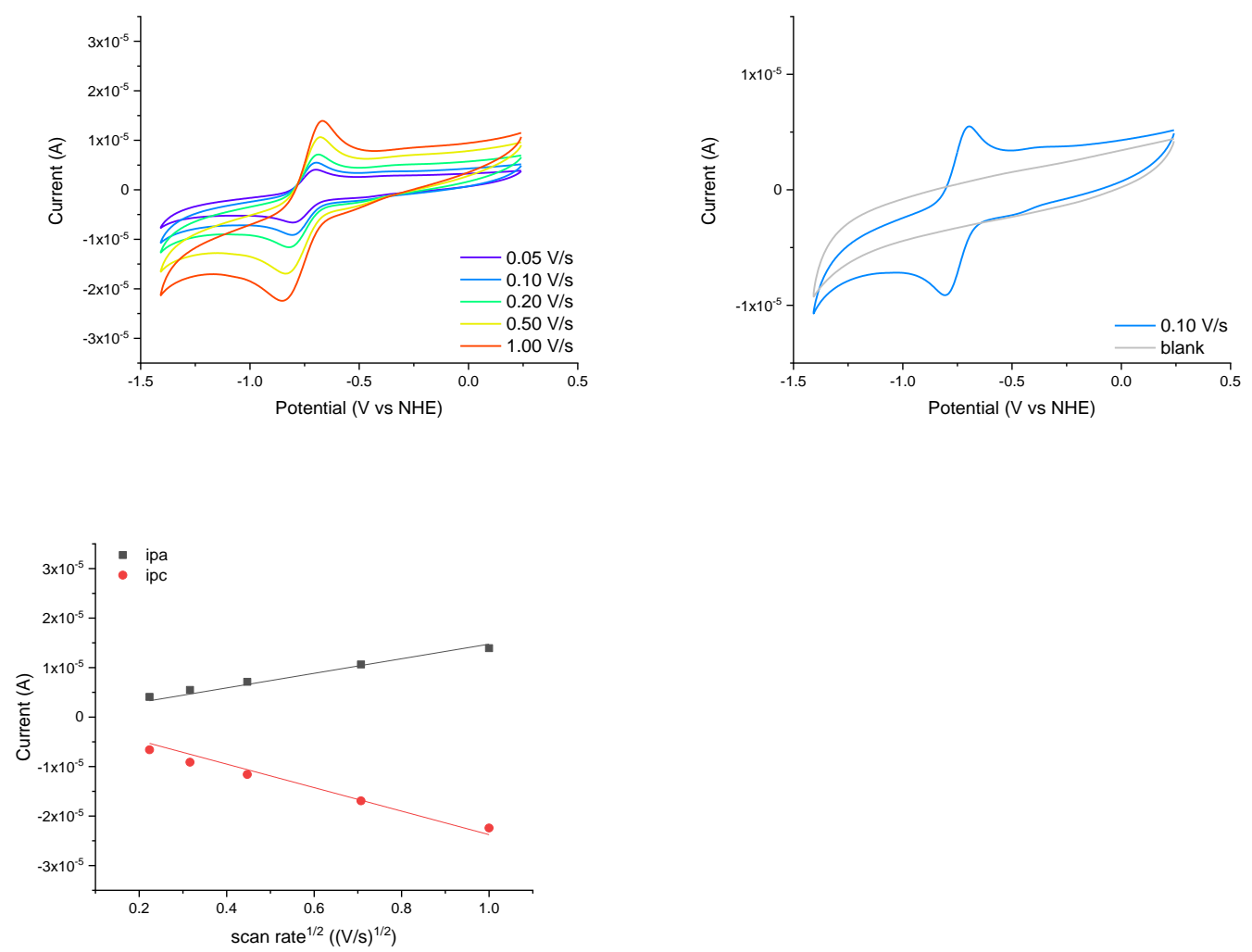

Figure S17. Cyclic voltammograms at various scan rate for EuL2 $2^{\text {MOM }}$ and plot of peak current intensities vs. square root of scan rate.

Table S9. Values for linear fit of peak current intensities $v s$. square root of scan rate for EuL2MOM.

\begin{tabular}{lll} 
Equation: $\mathbf{y}=\mathbf{a} * \mathbf{x}$ & ipa & ipc \\
\hline Slope & $1.47 \cdot 10^{-5} \pm 5.56 \cdot 10^{-7}$ & $-2.381 \cdot 10^{-5} \pm 9.75 \cdot 10^{-7}$ \\
R-Square (COD) & 0.99434 & 0.9933
\end{tabular}



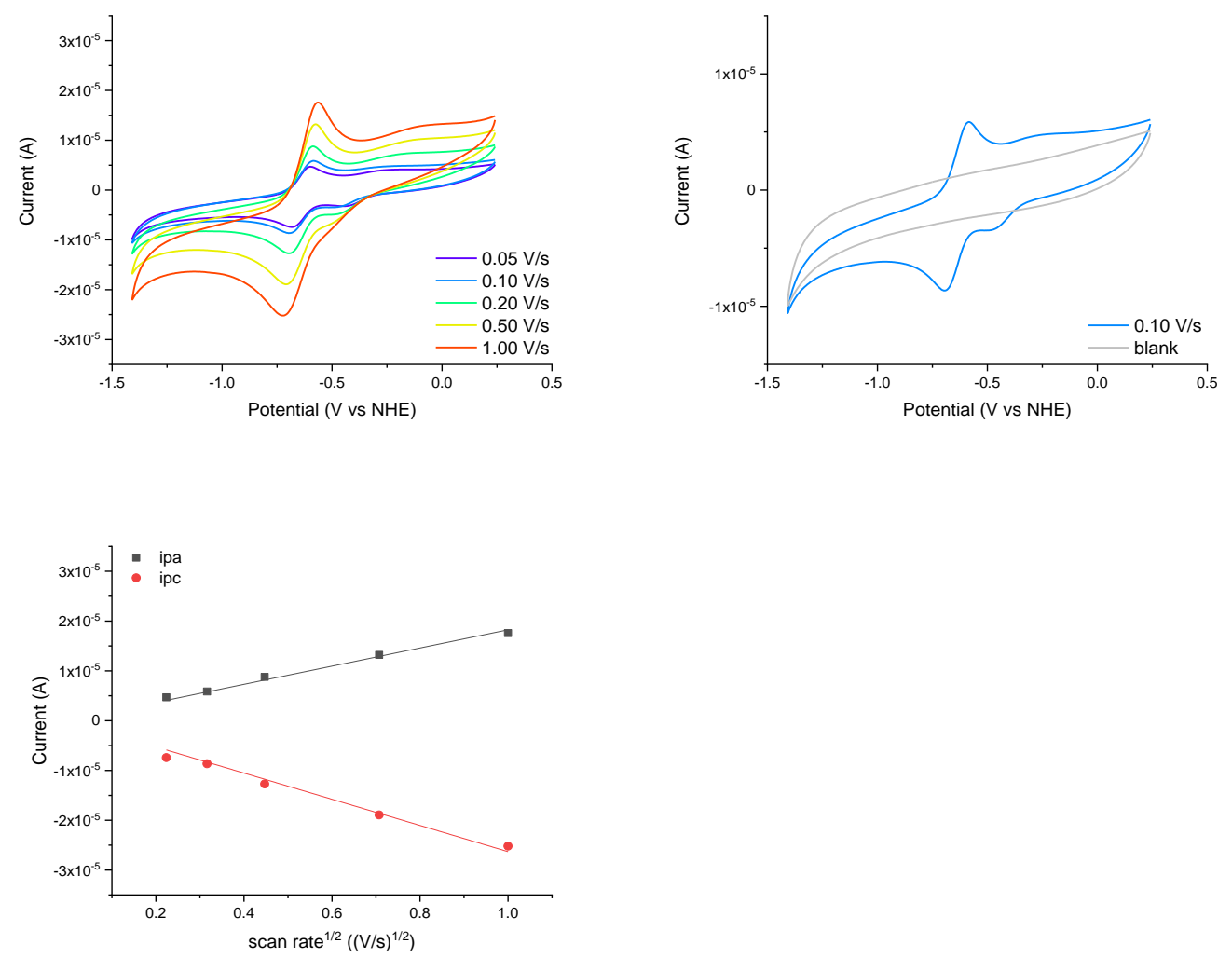

Figure S18. Cyclic voltammograms at various scan rate for EuL3 ${ }^{\text {MOM }}$ and plot of peak current intensities vs. square root of scan rate.

Table S10. Values for linear fit of peak current intensities $v s$. square root of scan rate for EuL3 ${ }^{\text {MOM. }}$

\begin{tabular}{lll} 
Equation: $\mathbf{y}=\mathbf{a}^{*} \mathbf{x}$ & ipa & ipc \\
\hline Slope & $1.82 \cdot 10^{-5} \pm 4.15 \cdot 10^{-7}$ & $-2.63 \cdot 10^{-5} \pm 7.97 \cdot 10^{-7}$ \\
R-Square (COD) & 0.99794 & 0.99634 \\
\hline
\end{tabular}



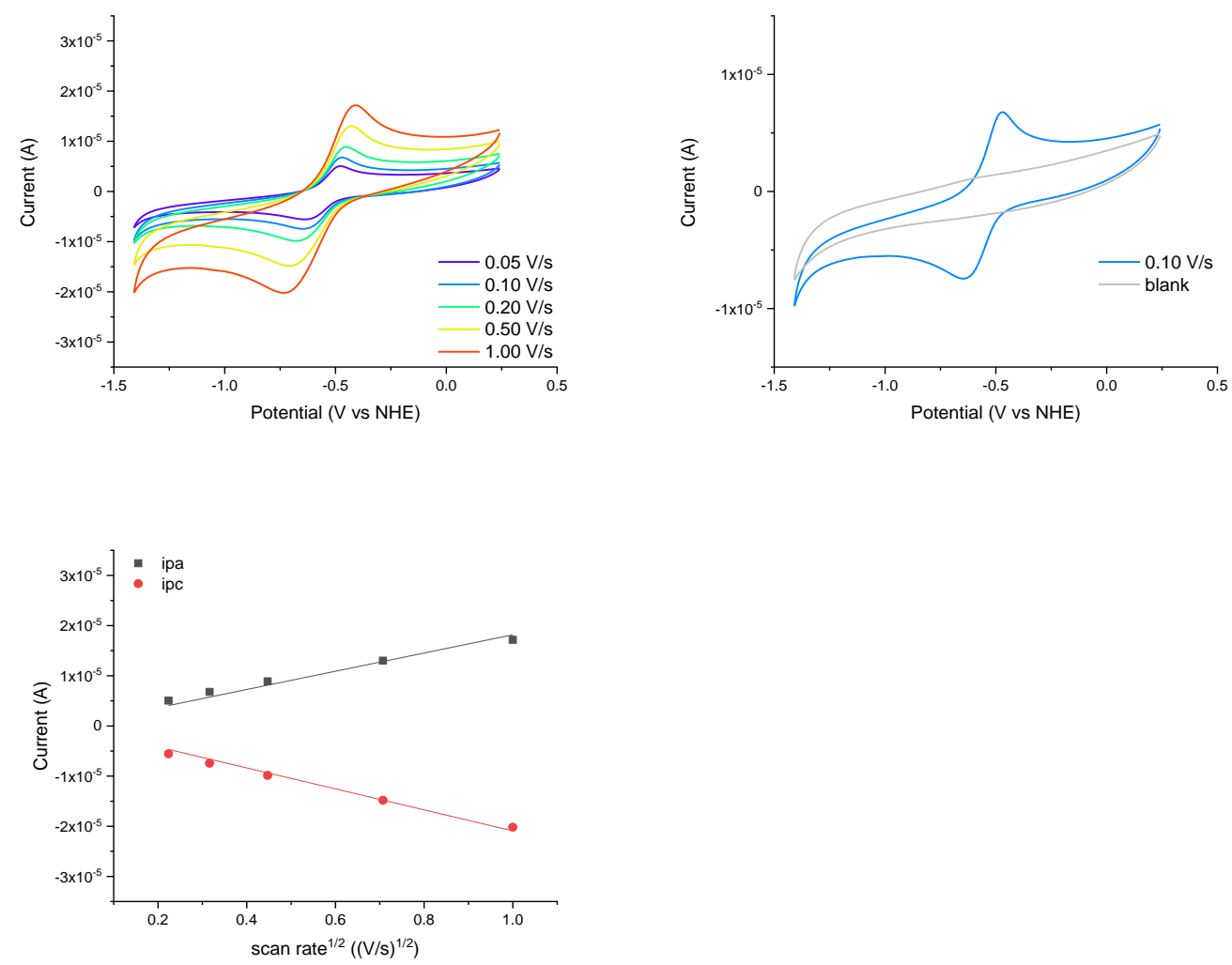

Figure S19. Cyclic voltammograms at various scan rate for EuL4a $\mathbf{A O M}^{\mathbf{M O}}$ and plot of peak current intensities vs. square root of scan rate.

Table S11. Values for linear fit of peak current intensities $v s$. square root of scan rate for EuL4aMom.

\begin{tabular}{lll} 
Equation: $\mathbf{y}=\mathbf{a}^{*} \mathbf{x}$ & ipa & ipc \\
\hline Slope & $1.82 \cdot 10^{-5} \pm 7.02 \cdot 10^{-7}$ & $-2.09 \cdot 10^{-5} \pm 5.49 \cdot 10^{-7}$ \\
R-Square (COD) & 0.99407 & 0.99726 \\
\hline
\end{tabular}



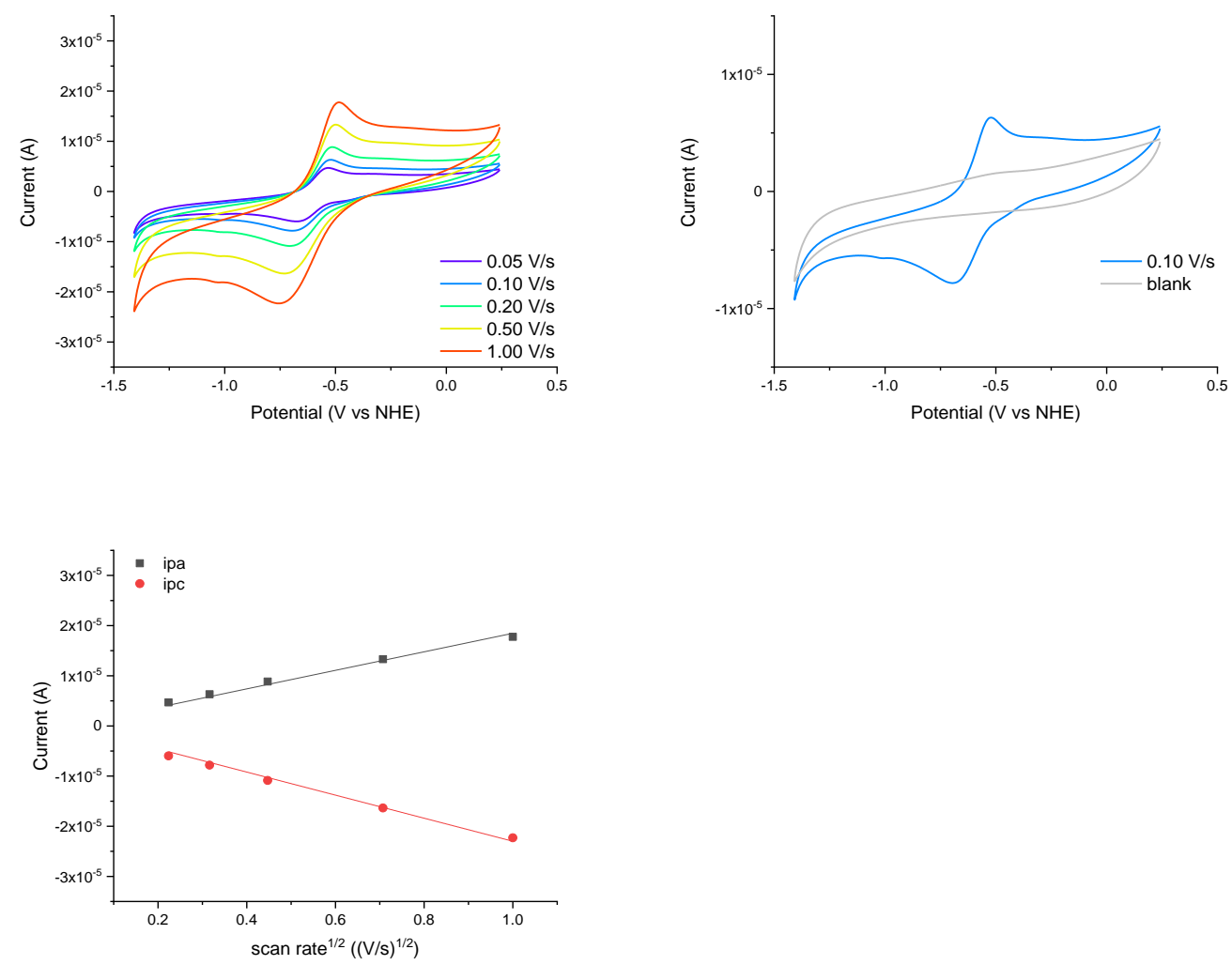

Figure S20. Cyclic voltammograms at various scan rate for EuL4b $\mathbf{b}^{\mathbf{M O M}}$ and plot of peak current intensities vs. square root of scan rate.

Table S12. Values for linear fit of peak current intensities $v s$. square root of scan rate for EuL4b'㠃.

\begin{tabular}{lll} 
Equation: $\mathbf{y}=\mathbf{a}^{*} \mathbf{x}$ & ipa & ipc \\
\hline Slope & $1.85 \cdot 10^{-5} \pm 4.42 \cdot 10^{-7}$ & $-2.30 \cdot 10^{-5} \pm 4.96 \cdot 10^{-7}$ \\
R-Square (COD) & 0.99771 & 0.99815 \\
\hline
\end{tabular}



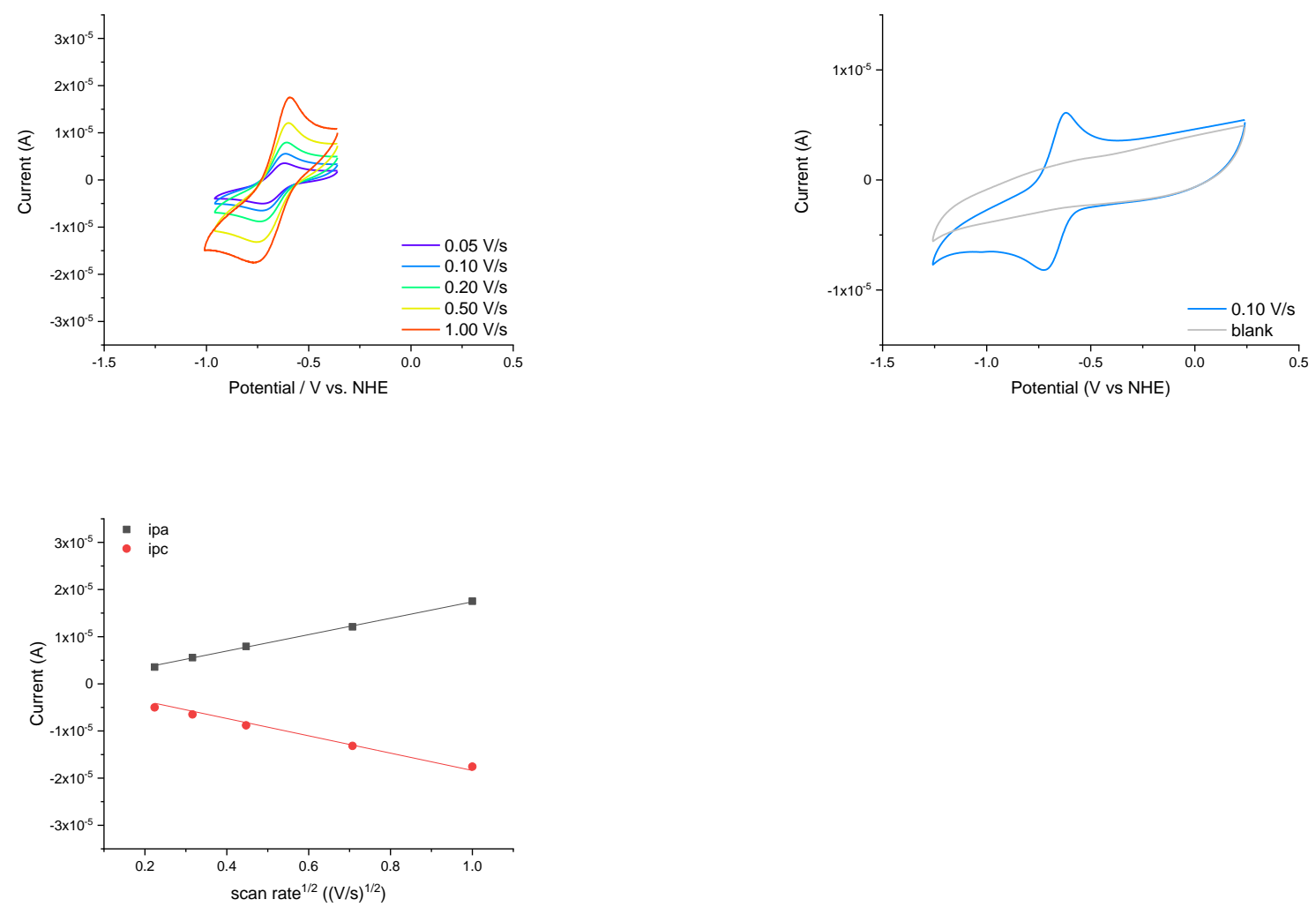

Figure S21. Cyclic voltammograms at various scan rate for $\mathbf{E u L 4} \mathbf{c}^{\mathbf{M O M}}$ and plot of peak current intensities vs. square root of scan rate.

Table S13. Values for linear fit of peak current intensities $v s$. square root of scan rate for EuL4c $\mathbf{c}^{\mathrm{MOM}}$.

\begin{tabular}{lll} 
Equation: $\mathbf{y}=\mathbf{a}^{*} \mathbf{x}$ & ipa & ipc \\
\hline Slope & $1.74 \cdot 10^{-5} \pm 1.66 \cdot 10^{-7}$ & $-1.84 \cdot 10^{-5} \pm 5.59 \cdot 10^{-7}$ \\
R-Square (COD) & 0.99963 & 0.99632 \\
\hline
\end{tabular}



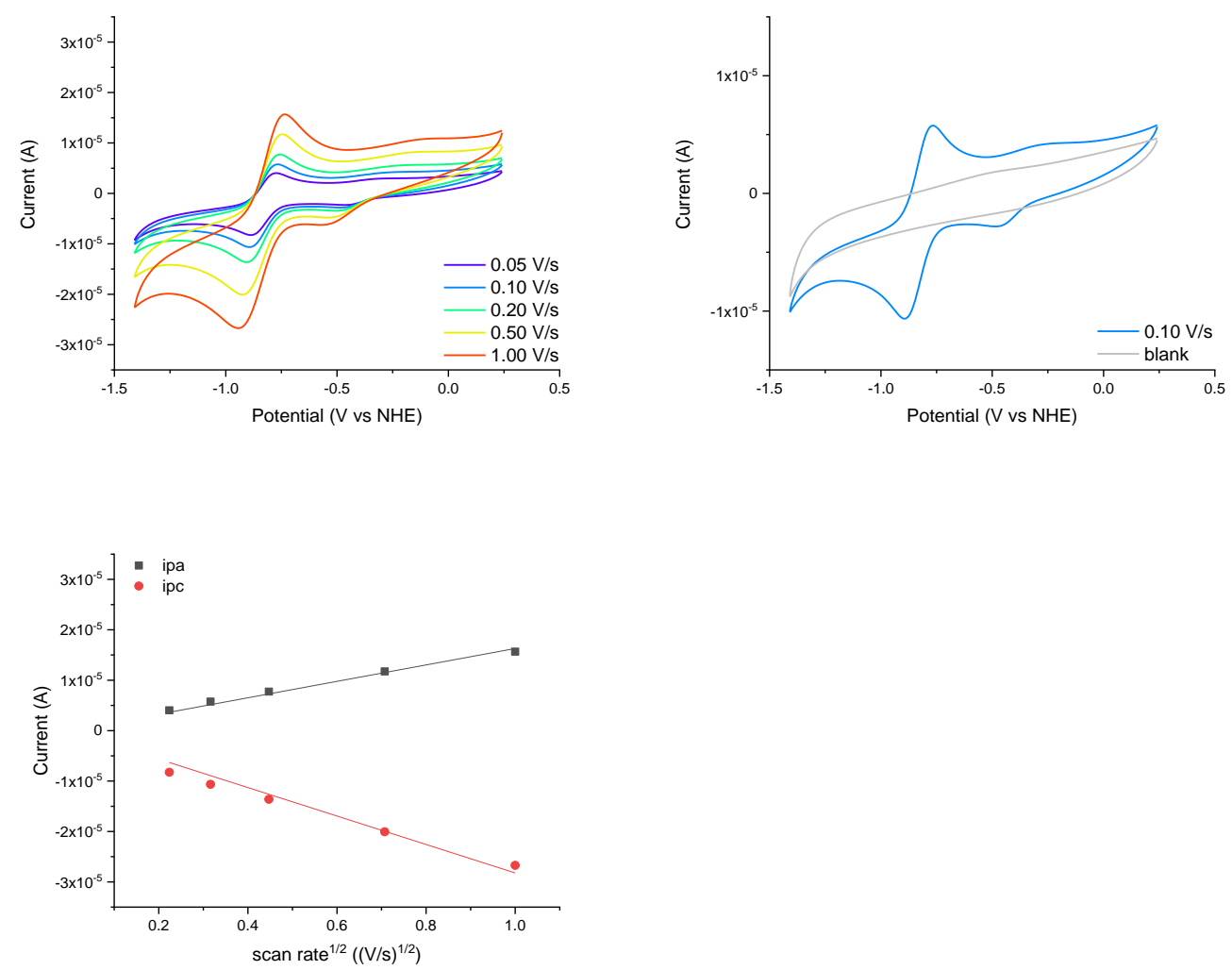

Figure S22. Cyclic voltammograms at various scan rate for $\mathbf{E u L 1}^{\mathrm{Me}}$ and plot of peak current intensities vs. square root of scan rate.

Table S14. Values for linear fit of peak current intensities $v s$. square root of scan rate for EuL1 ${ }^{M e}$.

\begin{tabular}{lll} 
Equation: $\mathbf{y}=\mathbf{a}^{*} \mathbf{x}$ & ipa & ipc \\
\hline Slope & $1.63 \cdot 10^{-5} \pm 3.94 \cdot 10^{-7}$ & $-2.82 \cdot 10^{-5} \pm 1.16 \cdot 10^{-6}$ \\
R-Square (COD) & 0.99767 & 0.99325 \\
\hline
\end{tabular}



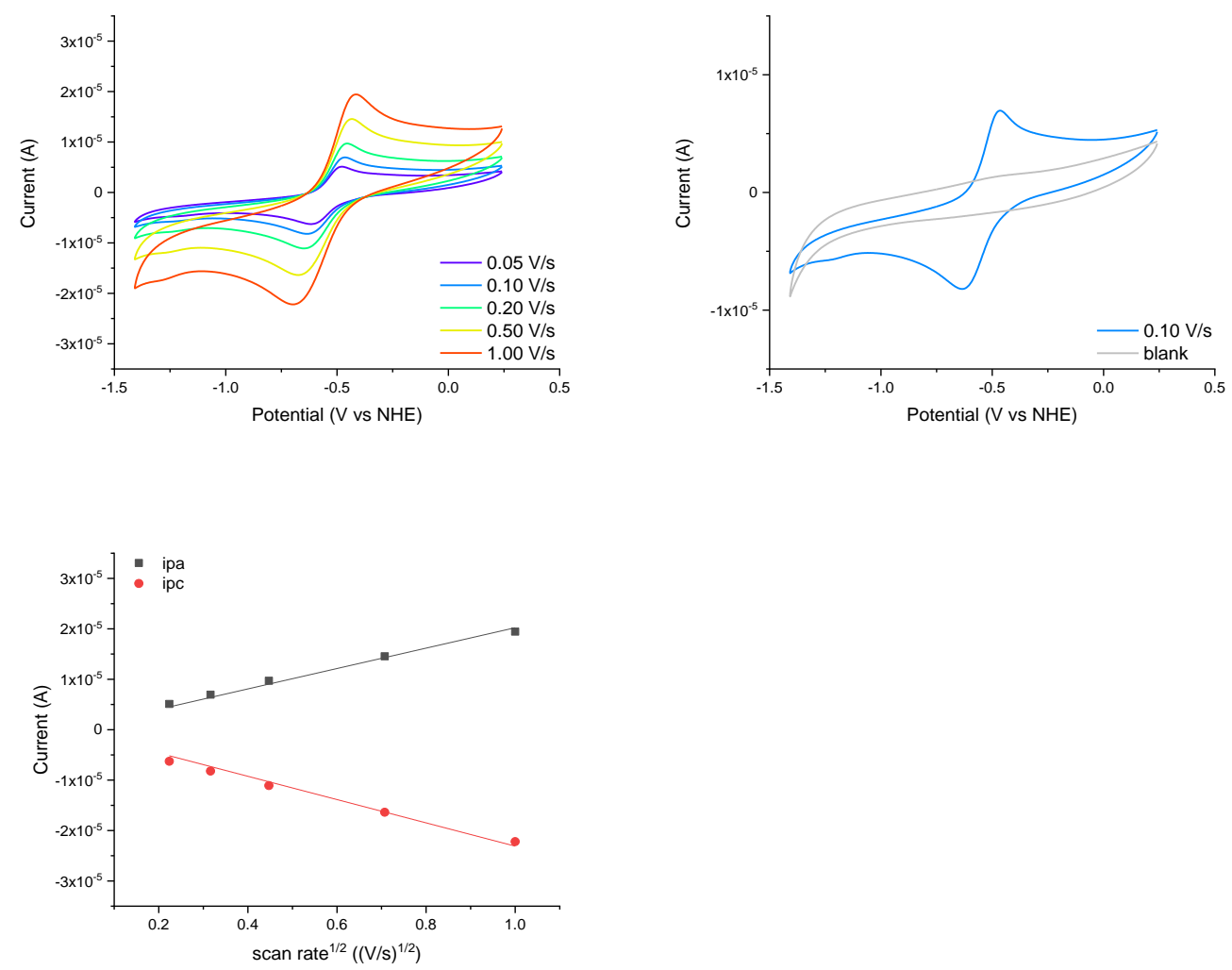

Figure S23. Cyclic voltammograms at various scan rate for $\mathbf{E u L 4 a} \mathbf{a}^{\mathrm{Me}}$ and plot of peak current intensities vs. square root of scan rate.

Table S15. Values for linear fit of peak current intensities vs. square root of scan rate for EuL4a $^{\mathrm{Me}}$.

\begin{tabular}{lll} 
Equation: $\mathbf{y}=\mathbf{a}^{*} \mathbf{x}$ & ipa & ipc \\
\hline Slope & $2.02 \cdot 10^{-5} \pm 4.88 \cdot 10^{-7}$ & $-2.31 \cdot 10^{-5} \pm 6.80 \cdot 10^{-7}$ \\
R-Square (COD) & 0.99768 & 0.99654
\end{tabular}



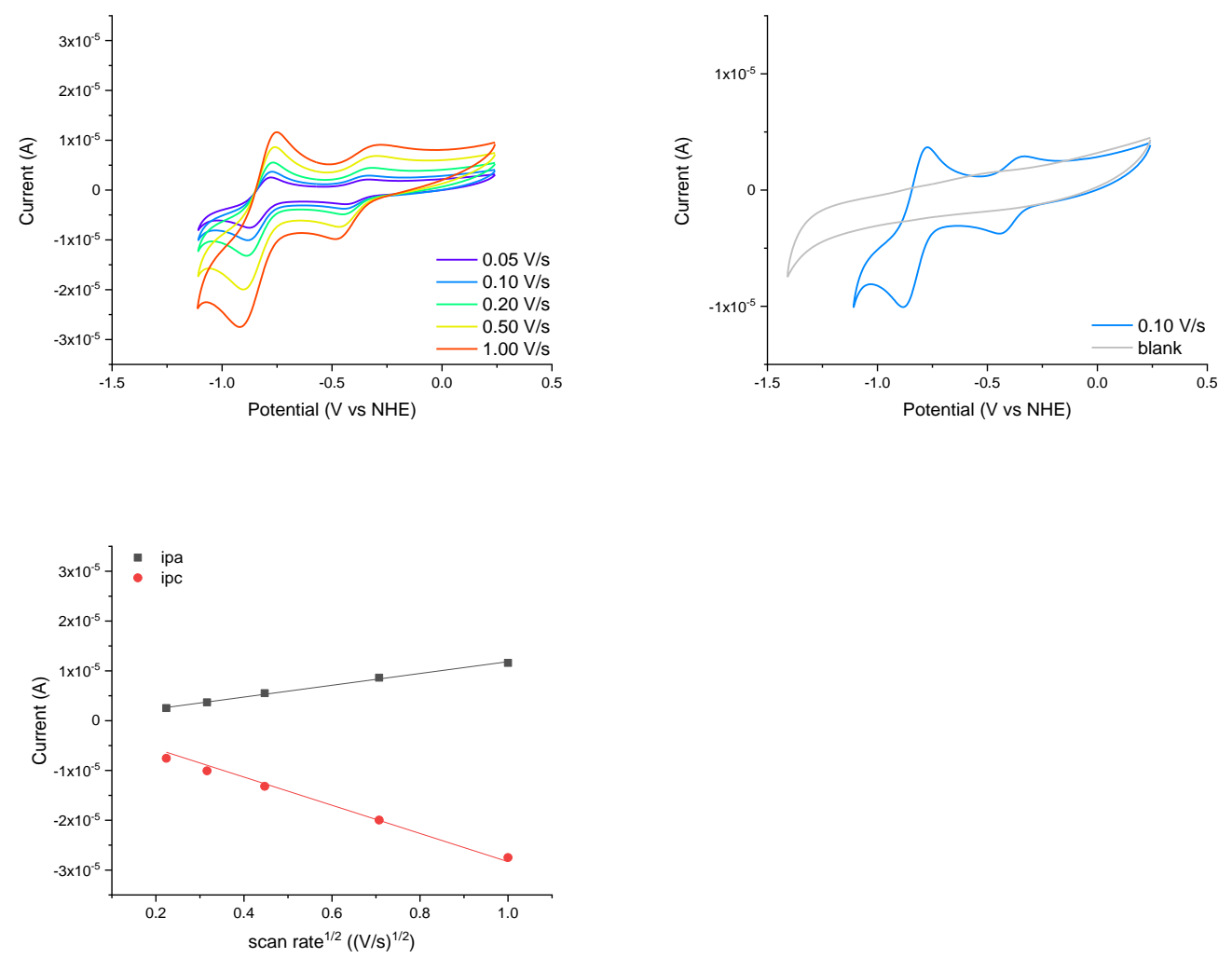

Figure S24. Cyclic voltammograms at various scan rate for EuL1 ${ }^{\mathbf{C F 3}}$ and plot of peak current intensities vs. square root of scan rate. Traces of $\mathrm{Eu}^{3+}(\mathrm{aq})$ are also present in this sample.

Table S16. Values for linear fit of peak current intensities $v s$. square root of scan rate for EuL1CF3.

\begin{tabular}{lll} 
Equation: $\mathbf{y}=\mathbf{a}^{*} \mathbf{x}$ & ipa & ipc \\
\hline Slope & $1.18 \cdot 10^{-5} \pm 1.62 \cdot 10^{-7}$ & $-2.83 \cdot 10^{-5} \pm 7.10 \cdot 10^{-7}$ \\
R-Square (COD) & 0.99925 & 0.99749
\end{tabular}



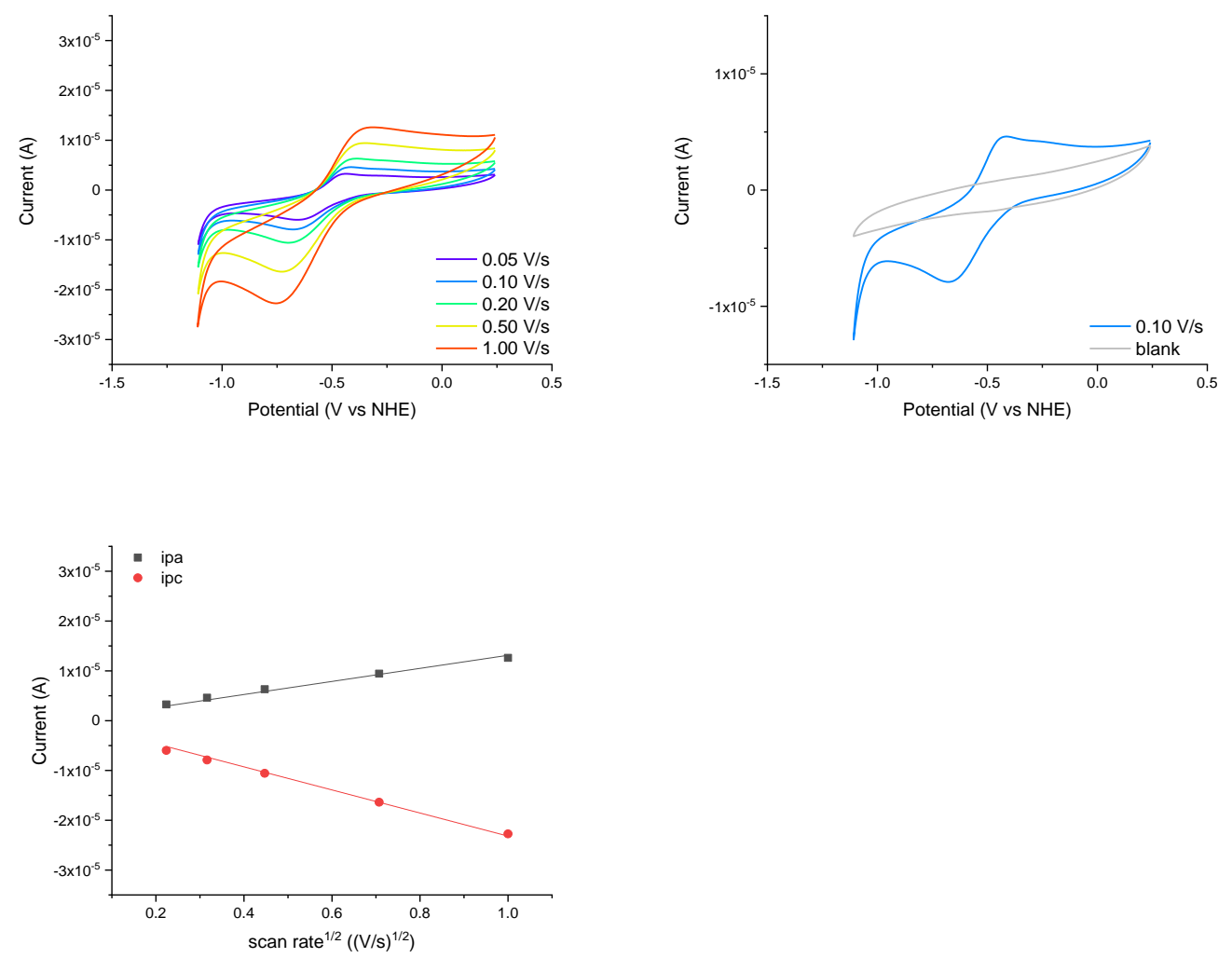

Figure S25. Cyclic voltammograms at various scan rate for EuL4a $\mathbf{C F}^{\mathbf{C F}}$ and plot of peak current intensities vs. square root of scan rate.

Table S17. Values for linear fit of peak current intensities $v s$. square root of scan rate for EuL4aCF3.

\begin{tabular}{lll} 
Equation: $\mathbf{y}=\mathbf{a}^{*} \mathbf{x}$ & ipa & ipc \\
\hline Slope & $1.31 \cdot 10^{-5} \pm 3.33 \cdot 10^{-7}$ & $-2.32 \cdot 10^{-5} \pm 4.00 \cdot 10^{-7}$ \\
R-Square (COD) & 0.99744 & 0.99881
\end{tabular}


Photophysical characterization
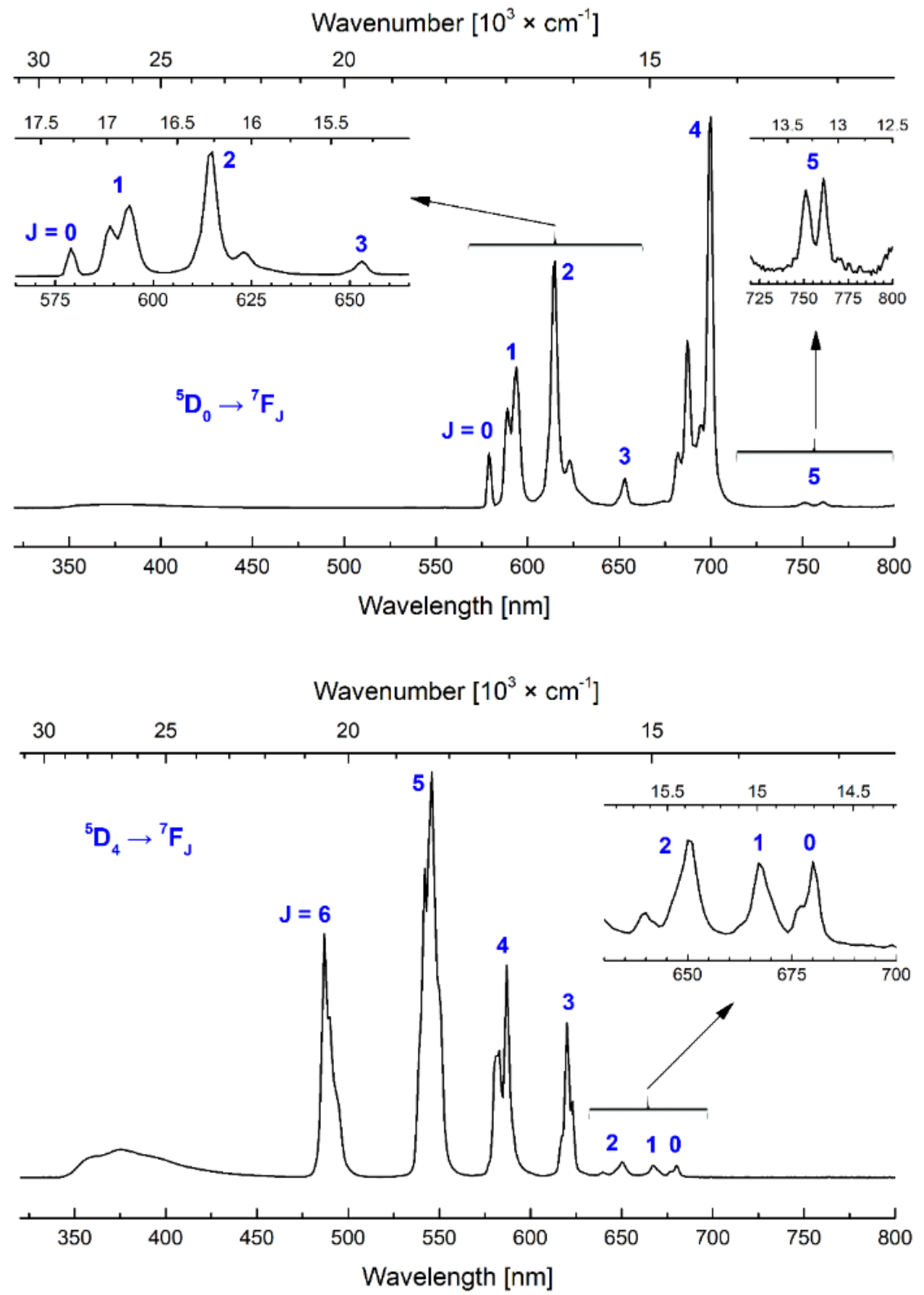

Figure S26. Steady-state emission spectra with the characteristic transitions of EuL3 $\mathbf{3}^{\mathbf{M O M}}$ (top) and $\mathbf{T b L 3}^{\text {MOM }}$ (bottom). [LnL3 $\left.{ }^{\text {MOM }}\right]=$ nominally $1.0 \times 10^{-5}$ M, PIPES buffered aqueous solutions $(0.01 \mathrm{M})$ at $\mathrm{pH} 6.5 ; \lambda_{\mathrm{ex}}=335 \mathrm{~nm}$. 


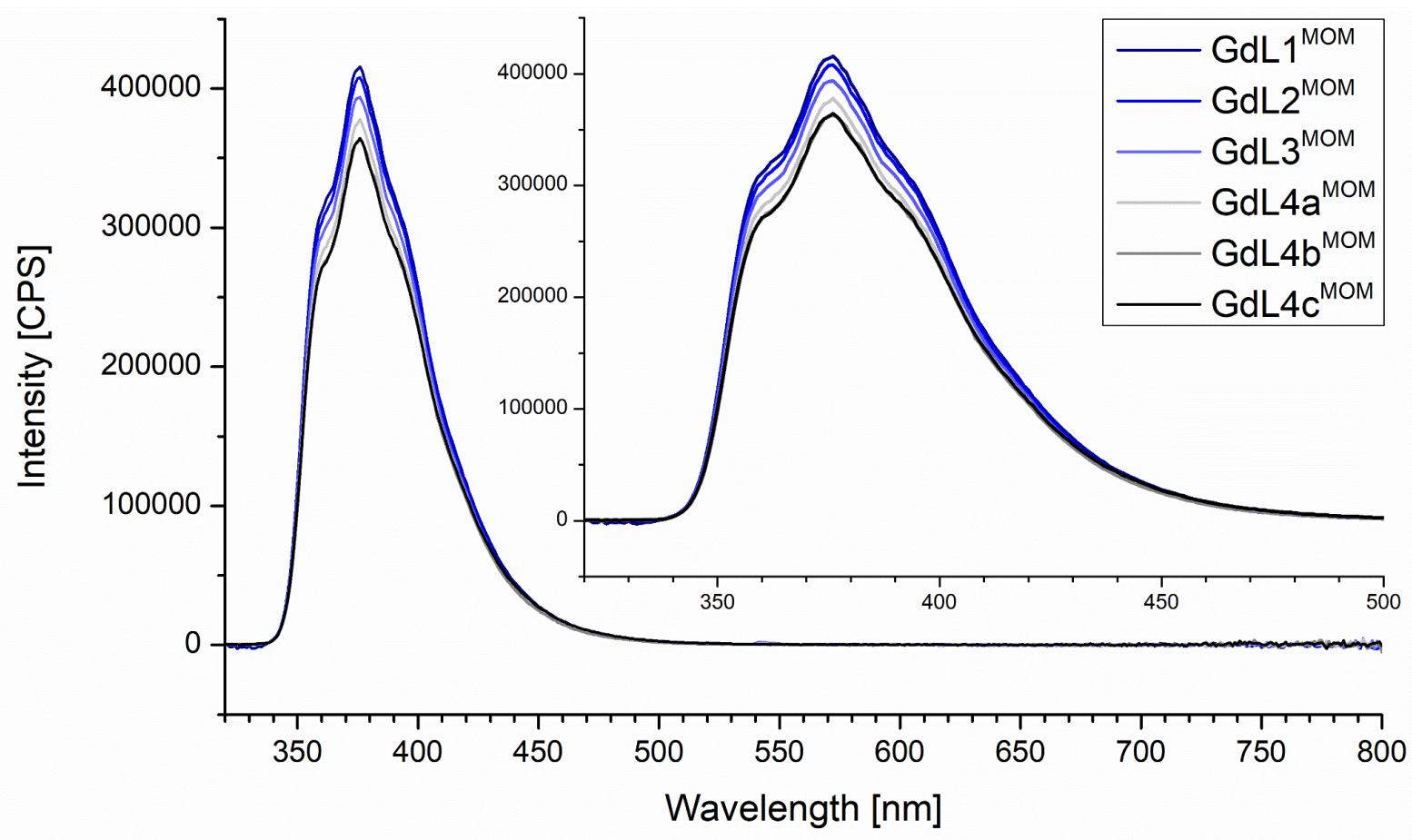

Figure S27. Fluorescence emission spectra of the GdL1-4c $\mathbf{c}^{\text {MOM }}$ showing the relative intensities under identical sample absorbance. $\lambda_{\mathrm{ex}}=330 \mathrm{~nm}$, Front slit: $2 \mathrm{~nm}$, Exit slit: $1 \mathrm{~nm}$.

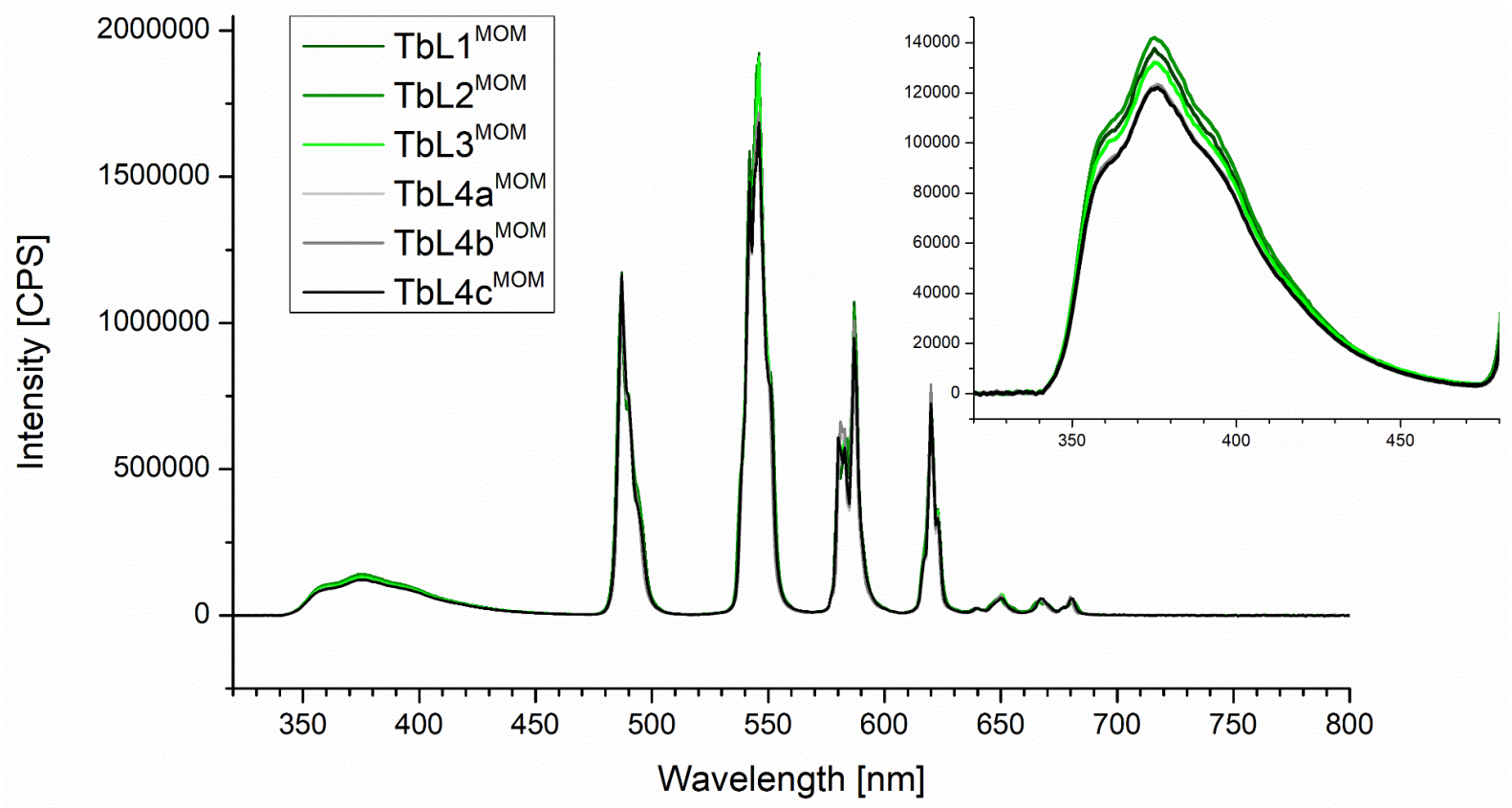

Figure S28. Fluorescence emission spectra of the TbL1-4c $\mathbf{c}^{\mathrm{MOM}}$ showing the relative intensities under identical sample absorbance. $\lambda_{\mathrm{ex}}=335 \mathrm{~nm}$, Front slit: $2 \mathrm{~nm}$, Exit slit: $1 \mathrm{~nm}$. 


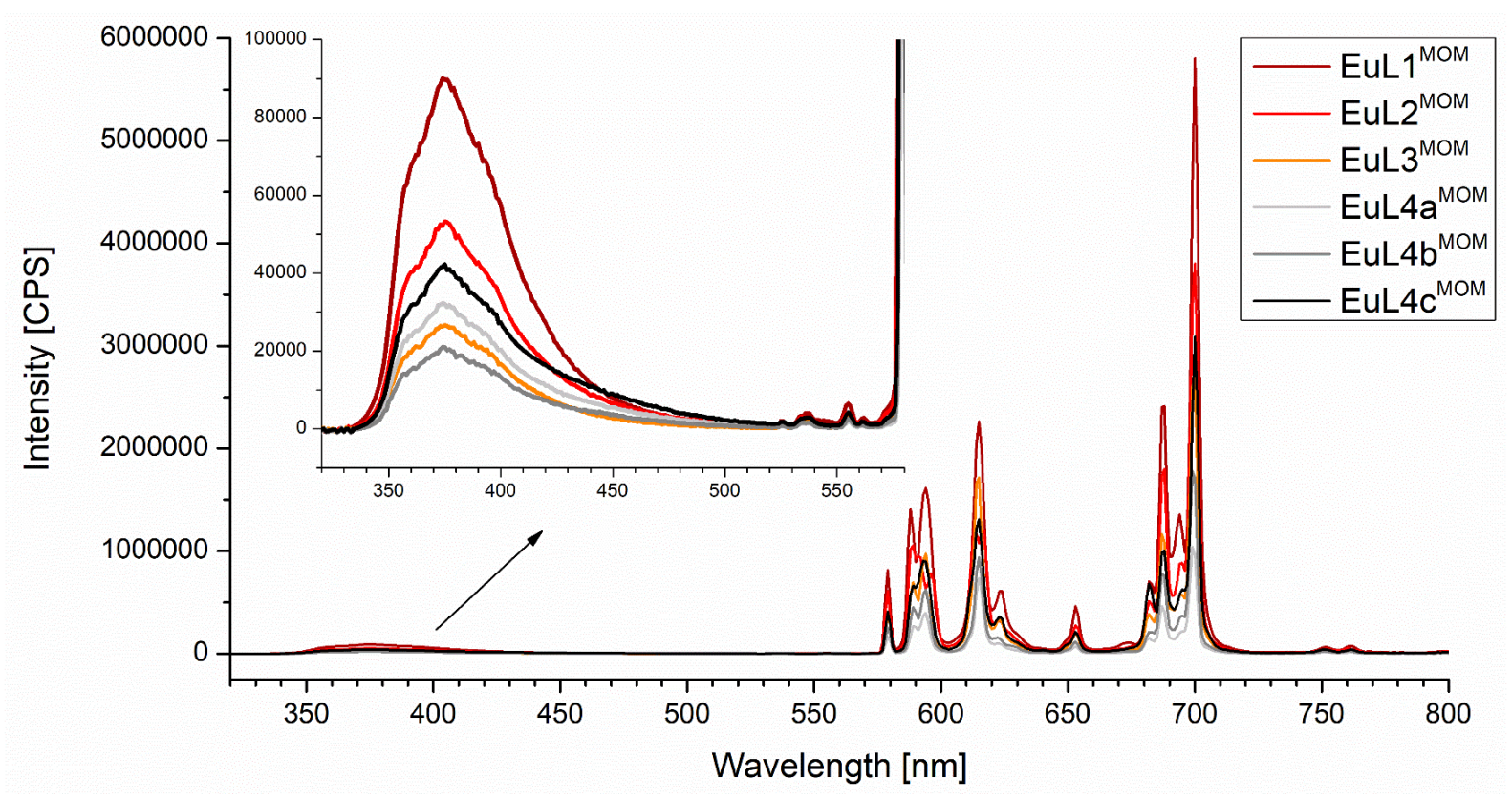

Figure S29. Fluorescence emission spectra of the EuL1-4c $\mathbf{c}^{\mathrm{MOM}}$ showing the relative intensities under identical sample absorbance. $\lambda_{\mathrm{ex}}=335 \mathrm{~nm}$, Front slit: $3 \mathrm{~nm}$, Exit slit: $2 \mathrm{~nm}$. 


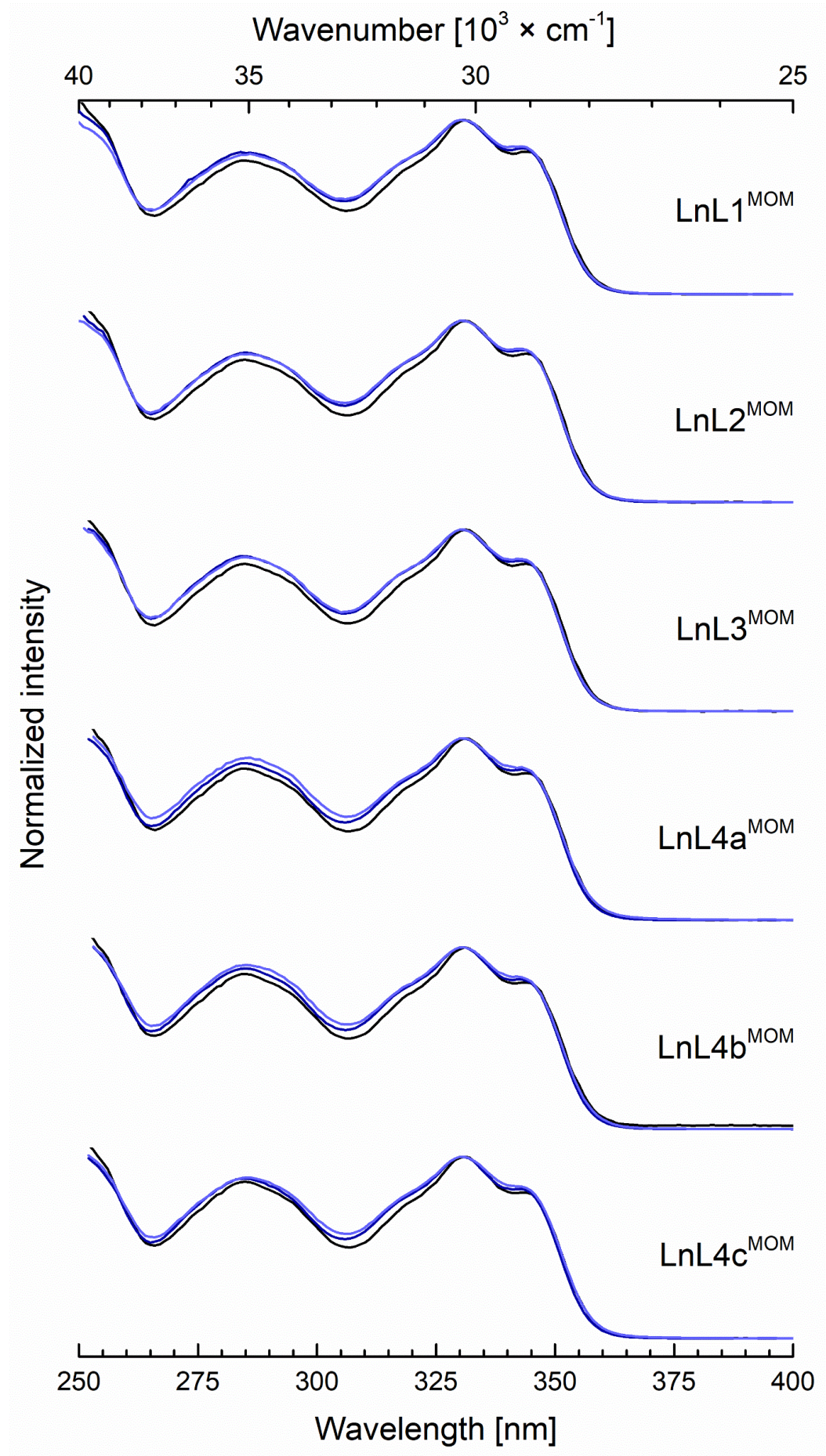

Figure S30. Superimposed absorption (black $-\mathrm{Gd}$ ) and excitation (deep blue $-\mathrm{Tb}, \lambda_{\mathrm{em}}=545$ $\mathrm{nm}$, light blue $\left.-\mathrm{Eu}, \lambda_{\mathrm{em}}=700 \mathrm{~nm}\right)$ spectra of $\mathbf{L n L 1}-\mathbf{4} \mathbf{c}^{\mathrm{MOM}}$. 


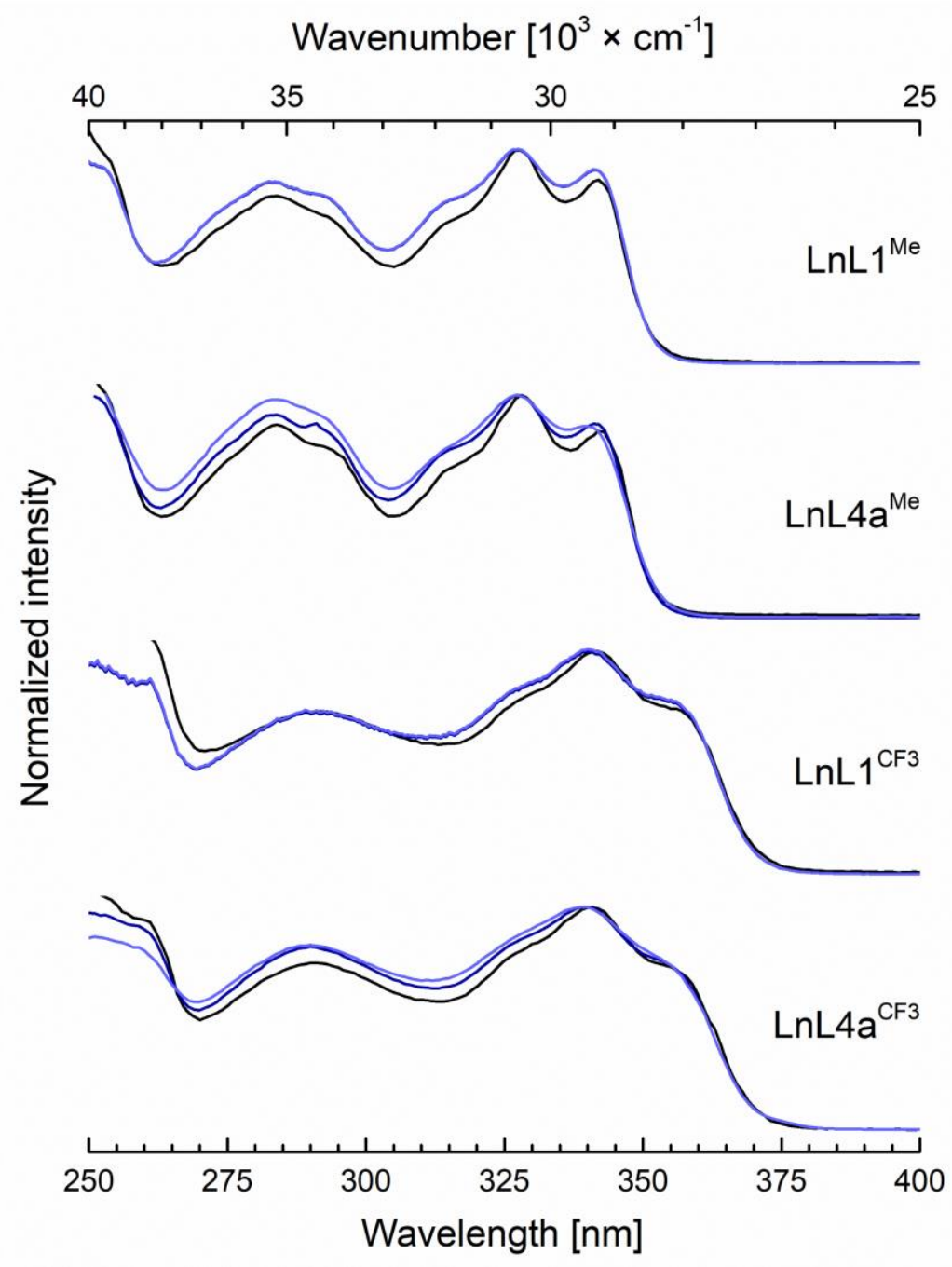

Figure S31. Overlaid absorption (black - Gd) and excitation (deep blue $-\mathrm{Tb}, \lambda_{\mathrm{em}}=545 \mathrm{~nm}$, light blue $\left.-\mathrm{Eu}, \lambda_{\mathrm{em}}=700 \mathrm{~nm}\right)$ spectra of $\mathbf{L n L 1} \mathbf{M e}^{\mathbf{M e}, \mathbf{C F}}$ and $\mathbf{L n L 4 a} \mathbf{a}^{\mathbf{M e}, \mathbf{C F 3}}$. 


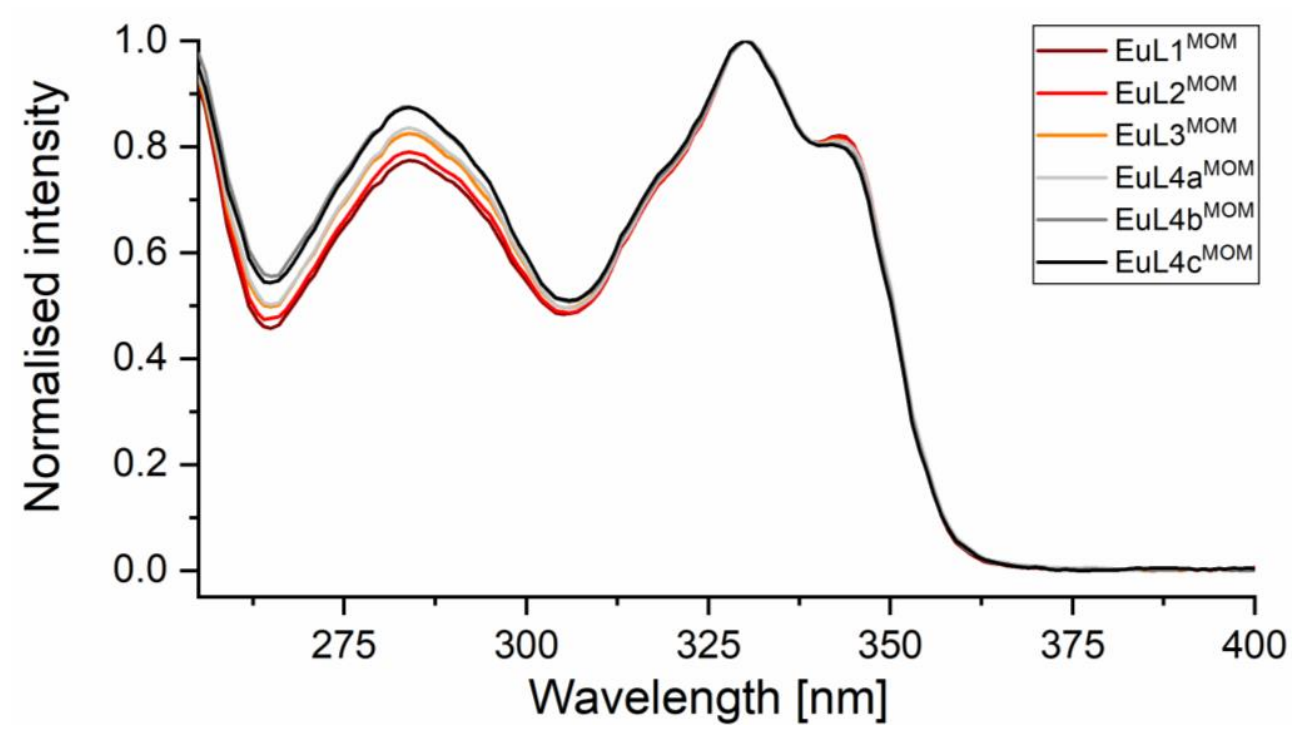

Figure S32. Superimposed UV absorption spectra of EuL1-4c $\mathbf{c}^{\text {MOM }}$ complexes in $0.01 \mathrm{M}$ PIPES-buffered aqueous solution, pH 6.5 normalized at $330 \mathrm{~nm}$.

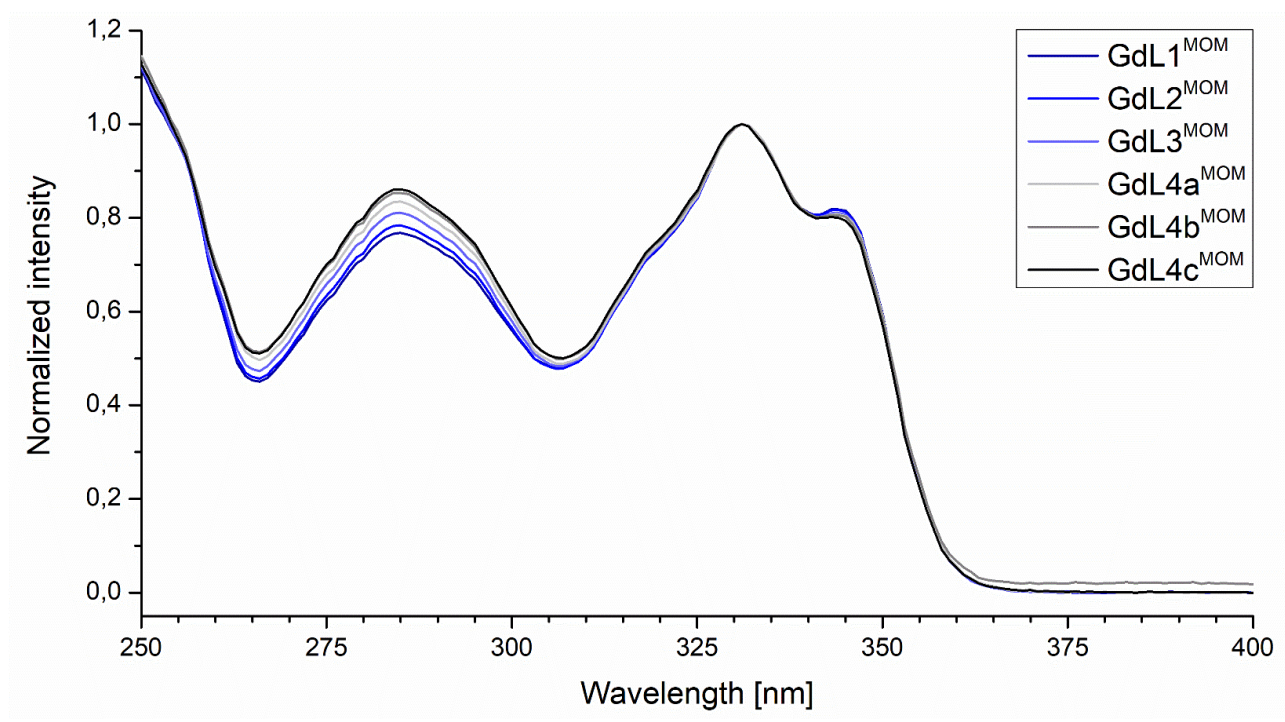

Figure S33. Superimposed UV absorption spectra of GdL1-4c $\mathbf{c}^{\text {MOM }}$ complexes in $0.01 \mathrm{M}$ PIPES-buffered aqueous solution, pH 6.5 normalized at $330 \mathrm{~nm}$. 


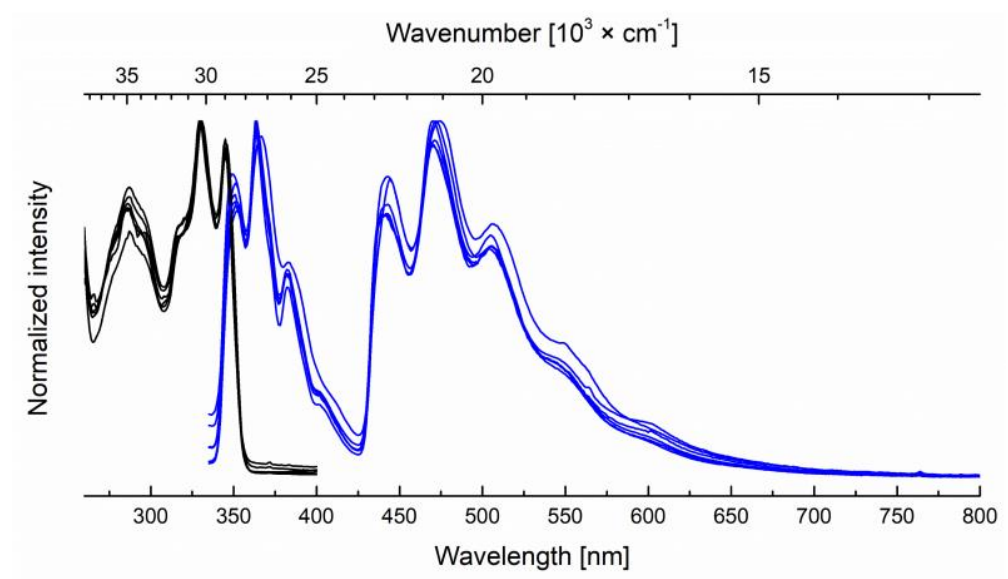

Figure S34. Overlaid excitation spectra of ligand-centered phosphorescence emissions of GdL1-4c MOM (black lines, left, $\lambda_{\mathrm{em}}=442 \mathrm{~nm}$ ), and their steady-state emission spectra (blue lines, right, $\lambda_{\text {exc }}=335 \mathrm{~nm}$ ) at $77 \mathrm{~K}$ with $10 \%$ glycerol added to the $10 \mu \mathrm{M}$, PIPES buffered aqueous solutions $0.01 \mathrm{M}, \mathrm{pH} 6.5$.

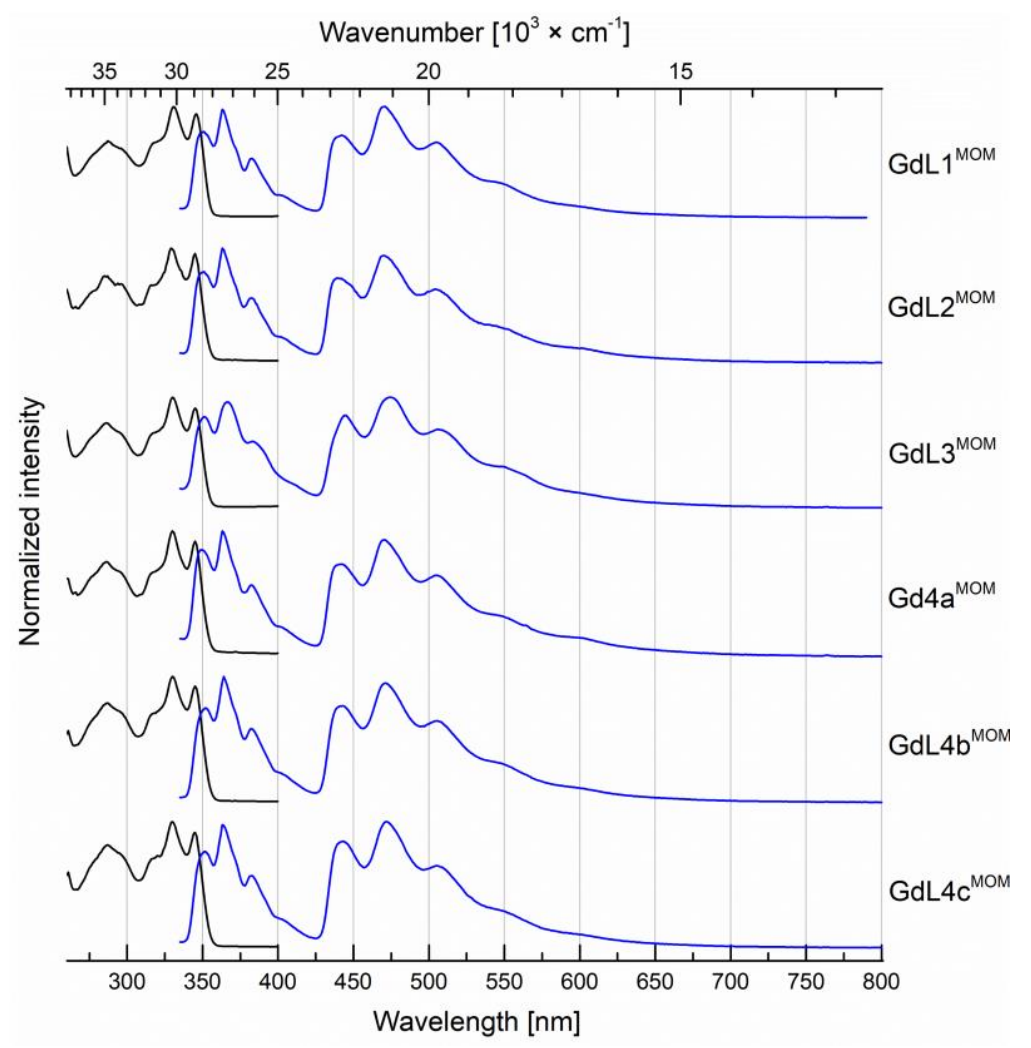

Figure S35. Excitation spectra of the ligand centered phosphorescence emissions of GdL1-4c $\mathbf{c}^{\text {MOM }}$ complexes (black lines, left, $\lambda_{\mathrm{em}}=442 \mathrm{~nm}$ ), and their steady-state emission spectra (blue lines, right, $\lambda_{\mathrm{ex}}=335 \mathrm{~nm}$ ) at $77 \mathrm{~K}$ with $10 \%$ glycerol added to the $10 \mu \mathrm{M}$, PIPES buffered aqueous solutions $0.01 \mathrm{M}, \mathrm{pH}$ 6.5. 


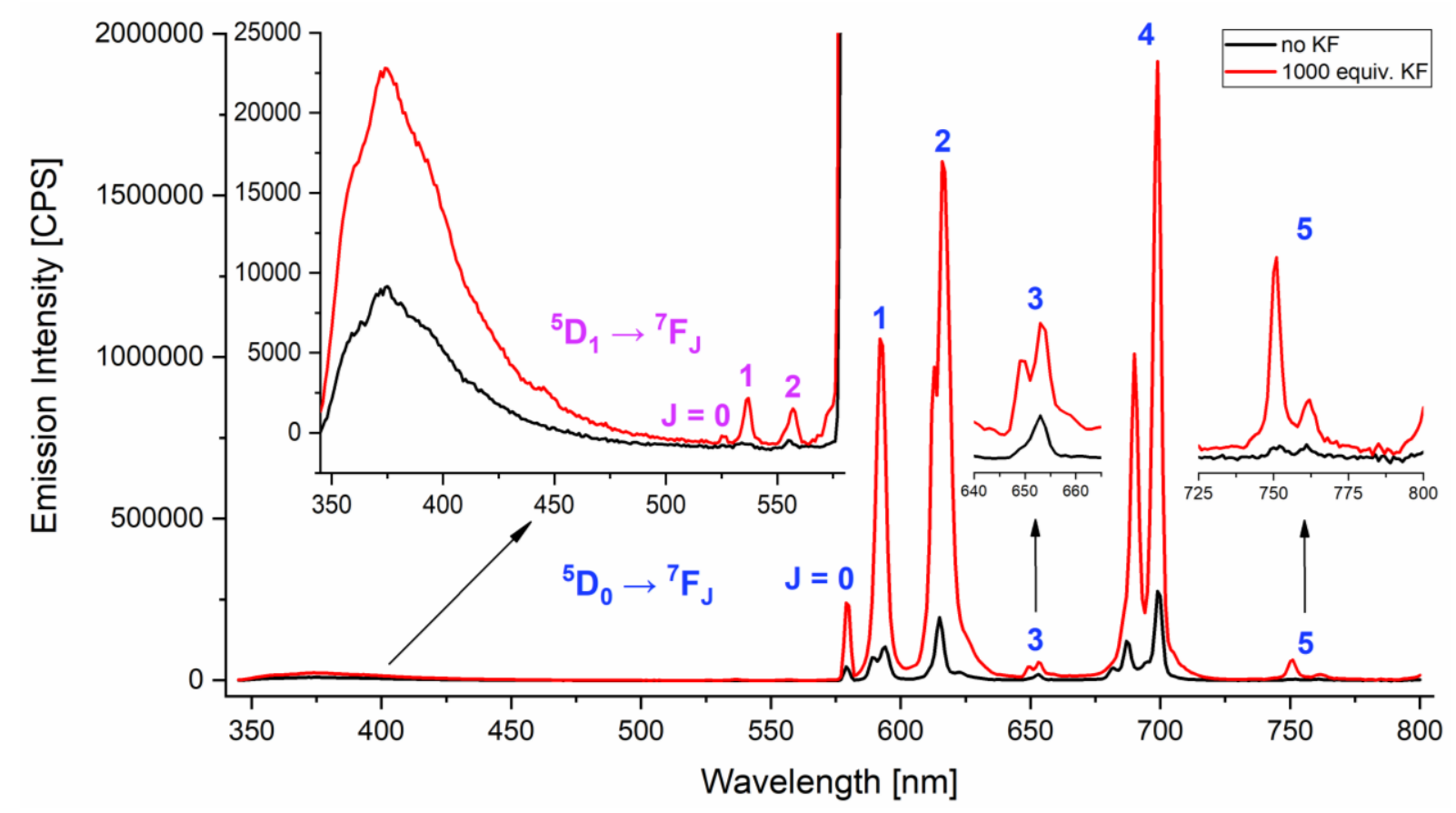

Figure S36. Steady-state emission spectra of EuL4a $\mathbf{a}^{\text {MOM }}$ with and without added excess KF under identical measurement setup. $\lambda_{\mathrm{ex}}=330 \mathrm{~nm}$, Front slit: $2 \mathrm{~nm}$, Exit slit: $2 \mathrm{~nm}$. 


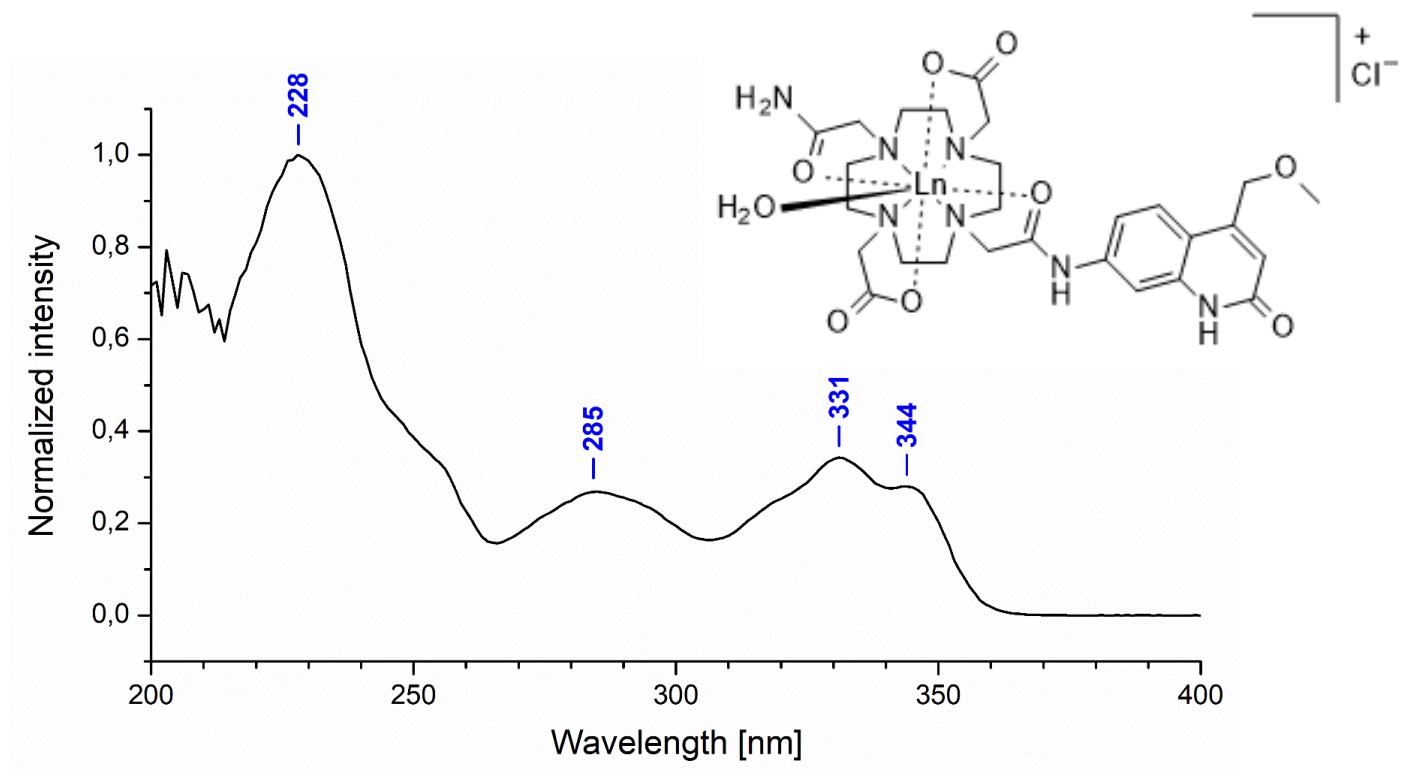

Figure S37. Normalized absorption spectrum of GdL2 $2^{\text {MOM }}$ in 0.01 M PIPES-buffered aqueous solution, pH 6.5. Blue numbers show local maxima.

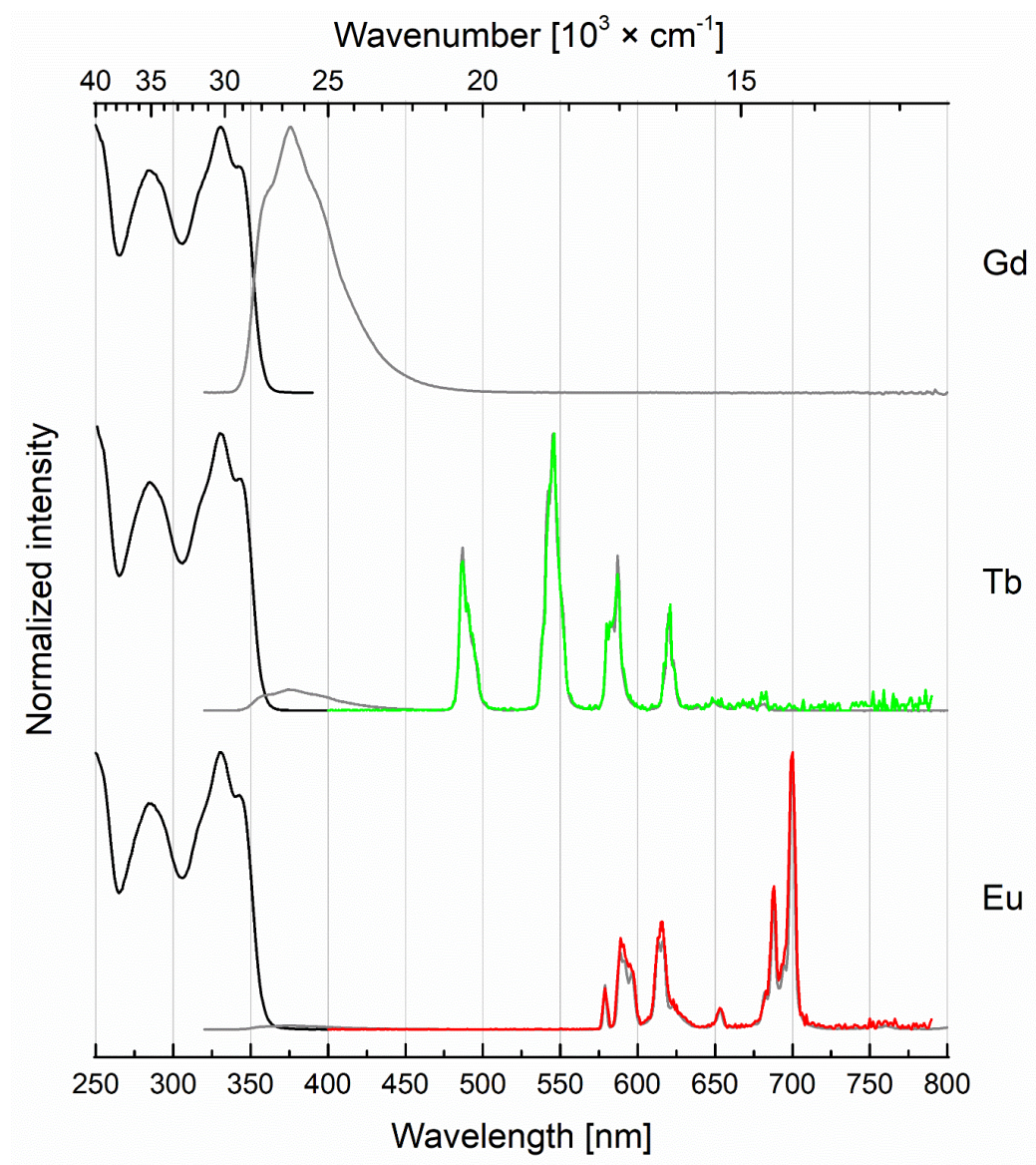

Figure S38. Excitation spectra of the Ln-centered emissions $\left(\mathrm{Tb}, \lambda_{\mathrm{em}}=545 \mathrm{~nm} ; \mathrm{Eu}, \lambda_{\mathrm{em}}=700\right.$ $\mathrm{nm})$ and the ligand centered emission (GdL2 $\left.{ }^{\mathbf{M O M}}, \lambda_{\mathrm{em}}=405 \mathrm{~nm}\right)$ at $298 \mathrm{~K}$ (black lines, left). Steady-state emission spectra of $\mathbf{L n L 2} \mathbf{2}^{\text {MOM }}$ at 298 K (grey lines, right) and time-resolved emission spectra showing the Ln-centered emissions ( $\mathrm{Tb}$ (green, $\lambda_{\mathrm{ex}}=335 \mathrm{~nm}, \lambda_{\mathrm{em}}=546 \mathrm{~nm}$ ), $\mathrm{Eu}\left(\mathrm{red}, \lambda_{\mathrm{ex}}=335 \mathrm{~nm}, \lambda_{\mathrm{em}}=615 \mathrm{~nm}\right), 298 \mathrm{~K}$, colored lines, right). 


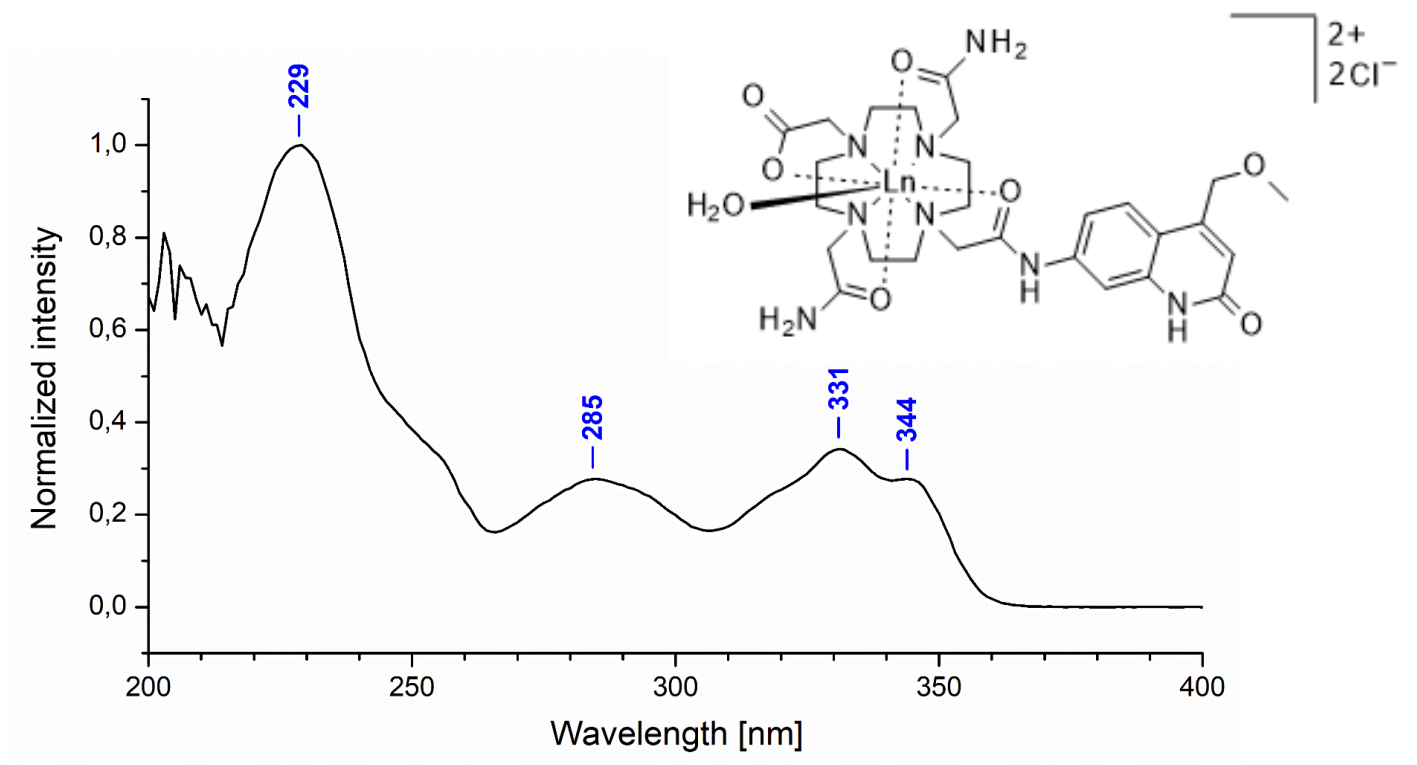

Figure S39. Normalized absorption spectrum of $\mathbf{G d L 3}^{\text {MOM }}$ in 0.01 M PIPES-buffered aqueous solution, pH 6.5. Blue numbers show local maxima.

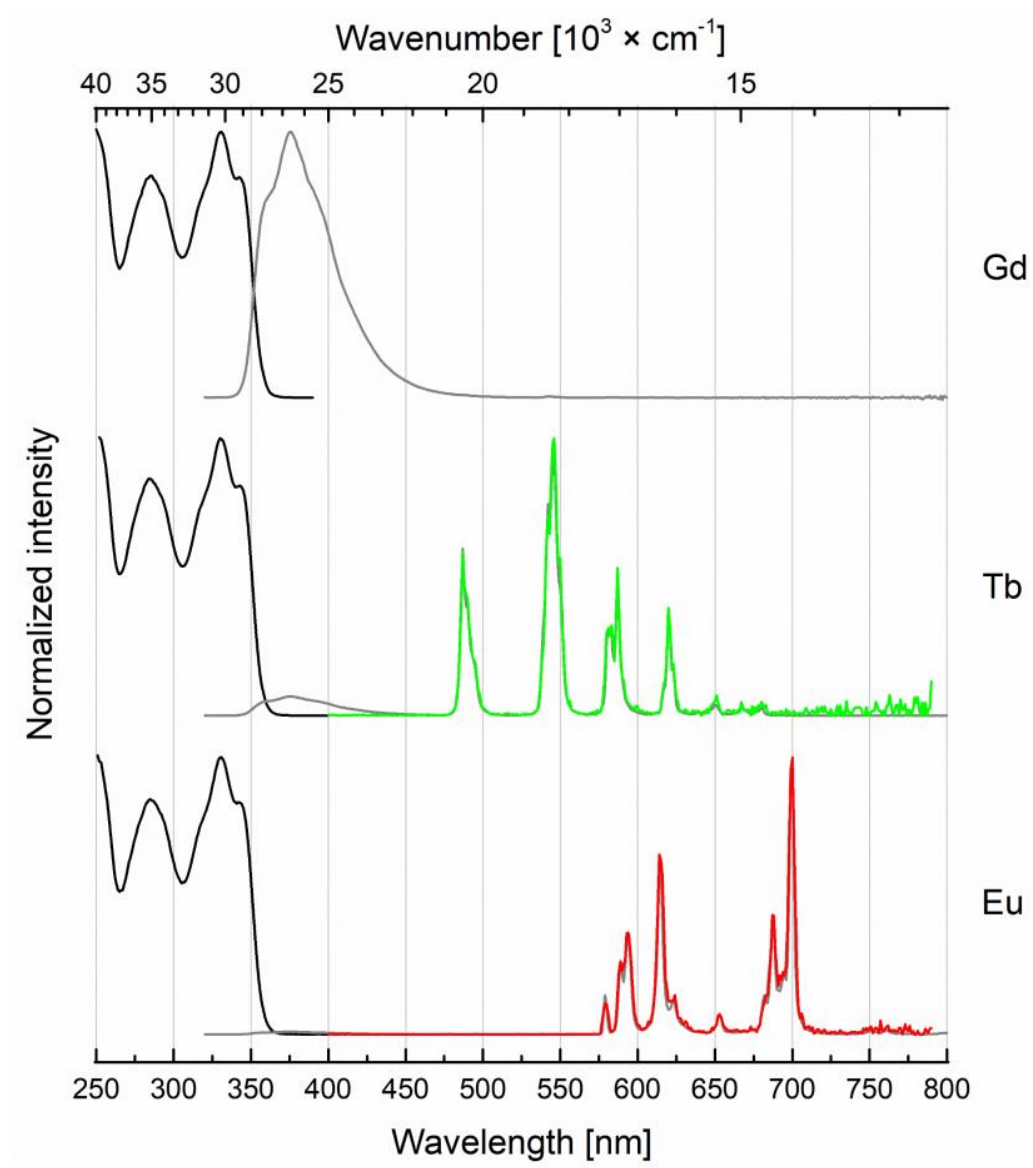

Figure S40. Excitation spectra of the Ln-centered emissions $\left(\mathrm{Tb}, \lambda_{\mathrm{em}}=545 \mathrm{~nm} ; \mathrm{Eu}, \lambda_{\mathrm{em}}=700\right.$ $\mathrm{nm})$ and of the ligand centered emission $\left(\mathbf{G d L 3}^{\mathbf{M O M}}, \lambda_{\mathrm{em}}=400 \mathrm{~nm}\right)$ at $298 \mathrm{~K}$ (black lines, left). Steady-state emission spectra of $\mathbf{L n L} 3^{\text {MOM }}$ at 298 K (grey lines, right) and time-resolved emission spectra showing the Ln-centered emissions ( $\mathrm{Tb}$ (green, $\lambda_{\mathrm{ex}}=335 \mathrm{~nm}, \lambda_{\mathrm{em}}=546 \mathrm{~nm}$ ), $\mathrm{Eu}\left(\mathrm{red}, \lambda_{\mathrm{ex}}=335 \mathrm{~nm}, \lambda_{\mathrm{em}}=615 \mathrm{~nm}\right), 298 \mathrm{~K}$, colored lines, right). 


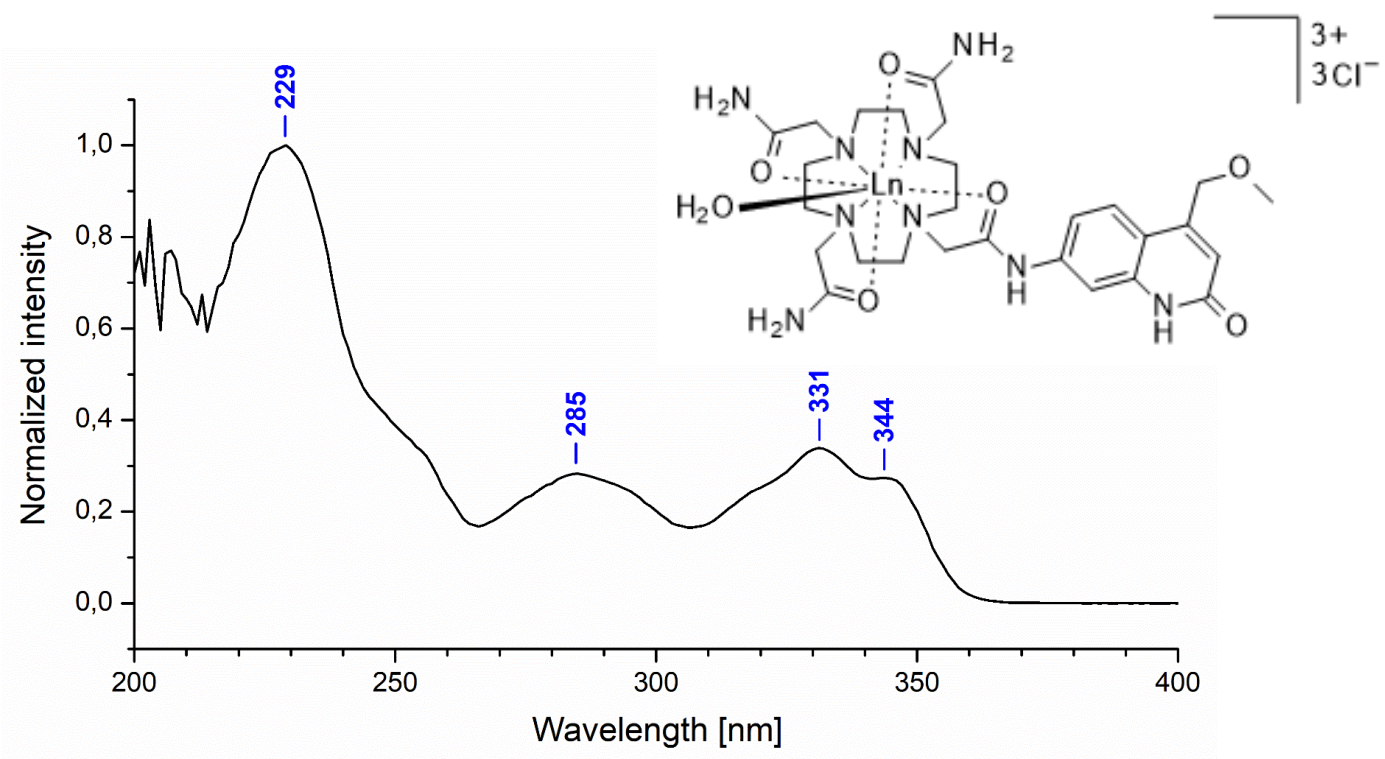

Figure S41. Normalized absorption spectrum of GdL4a $\mathbf{a}^{\text {MOM }}$ in 0.01 M PIPES-buffered aqueous solution, $\mathrm{pH}$ 6.5. Blue numbers show local maxima.

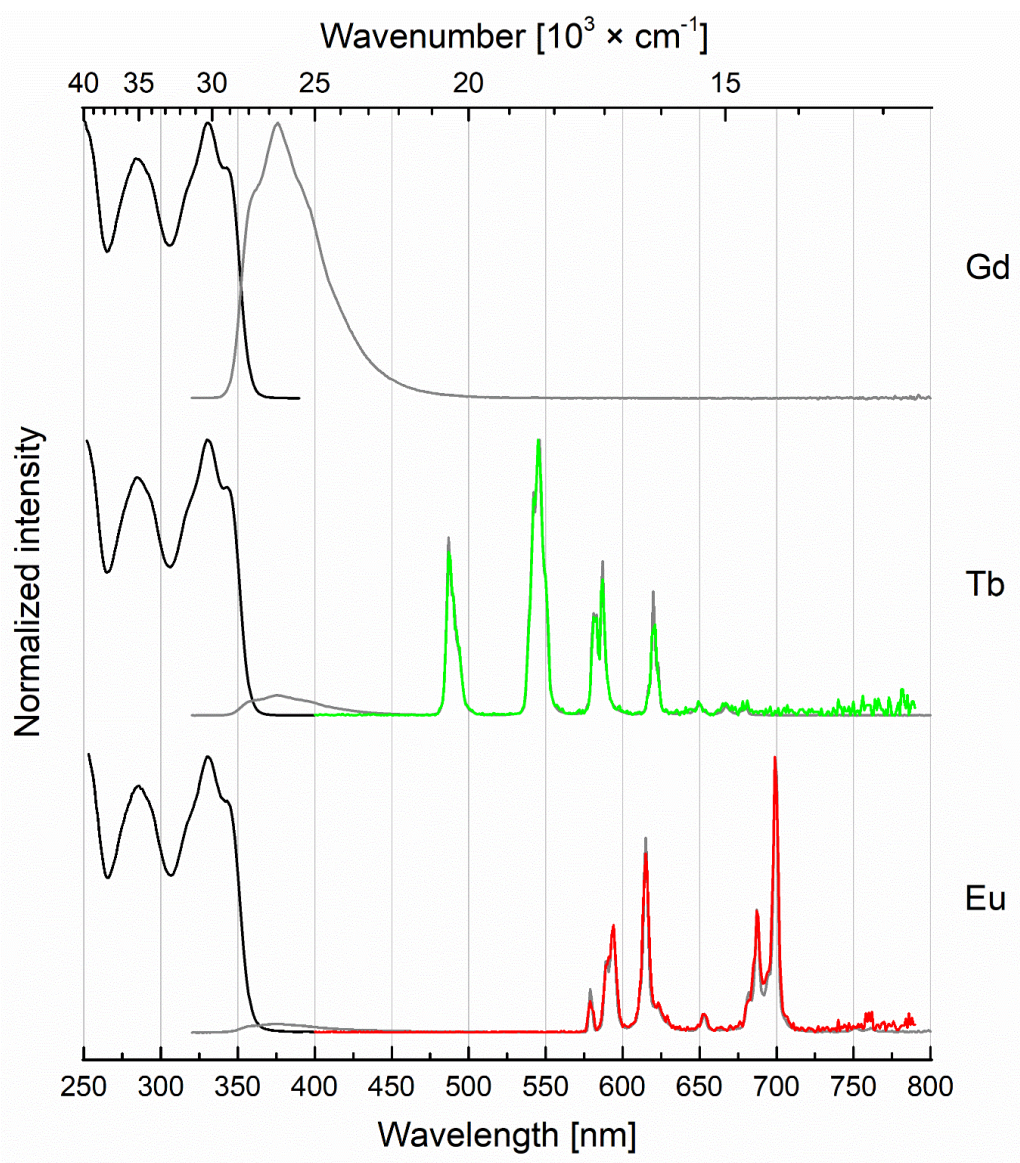

Figure S42. Excitation spectra of the Ln-centered emissions $\left(\mathrm{Tb}, \lambda_{\mathrm{em}}=545 \mathrm{~nm} ; \mathrm{Eu}, \lambda_{\mathrm{em}}=700\right.$ $\mathrm{nm})$ and of the ligand centered emission $\left(\mathbf{G d L 4} \mathbf{a}^{\mathbf{M O M}}, \lambda_{\mathrm{em}}=400 \mathrm{~nm}\right)$ at $298 \mathrm{~K}$ (black lines,

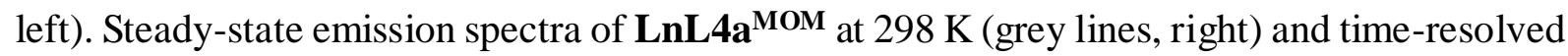
emission spectra showing the Ln-centered emissions ( $\mathrm{Tb}$ (green, $\lambda_{\mathrm{ex}}=335 \mathrm{~nm}, \lambda_{\mathrm{em}}=546 \mathrm{~nm}$ ), $\mathrm{Eu}\left(\mathrm{red}, \lambda_{\mathrm{ex}}=335 \mathrm{~nm}, \lambda_{\mathrm{em}}=615 \mathrm{~nm}\right), 298 \mathrm{~K}$, colored lines, right). 


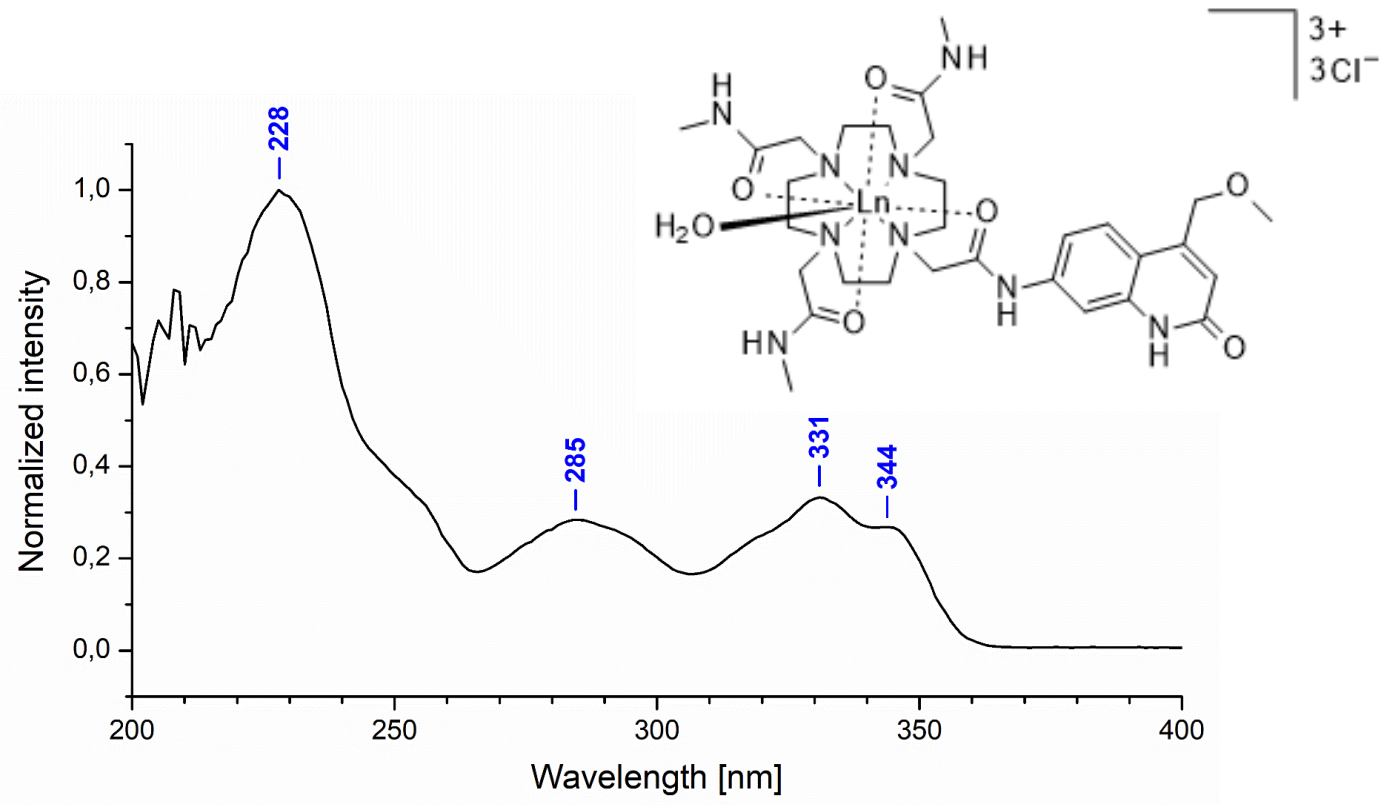

Figure S43. Normalized absorption spectrum of GdL4b $\mathbf{b}^{\text {MOM }}$ in 0.01 M PIPES-buffered aqueous solution, $\mathrm{pH}$ 6.5. Blue numbers show local maxima.

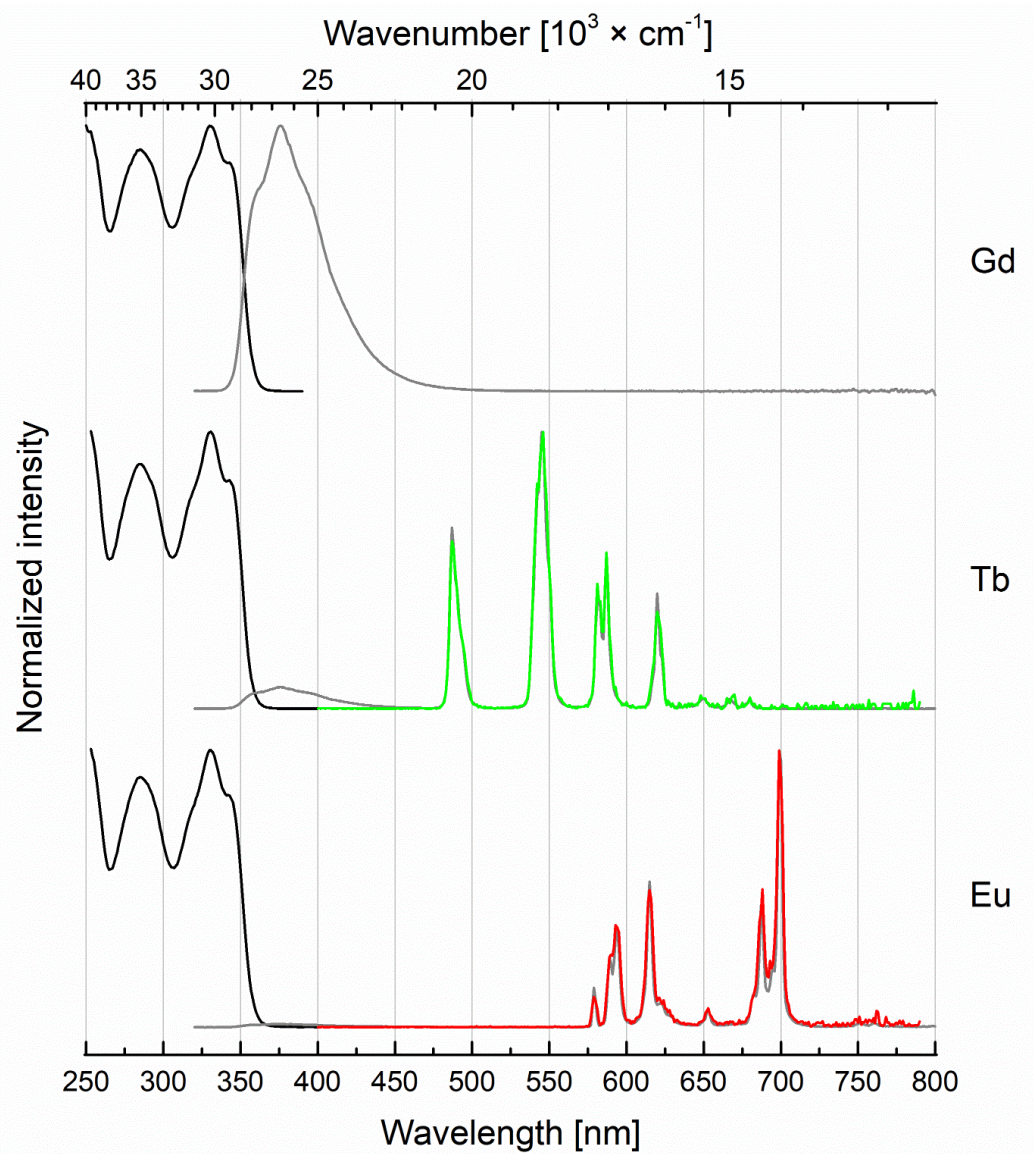

Figure S44. Excitation spectra of the Ln-centered emissions $\left(\mathrm{Tb}, \lambda_{\mathrm{em}}=545 \mathrm{~nm} ; \mathrm{Eu}, \lambda_{\mathrm{em}}=700\right.$ $\mathrm{nm})$ and of the ligand centered emission $\left(\mathbf{G d L 4} \mathbf{b}^{\mathrm{MOM}}, \lambda_{\mathrm{em}}=405 \mathrm{~nm}\right)$ at $298 \mathrm{~K}$ (black lines, left). Steady-state emission spectra of $\mathbf{L n L} \mathbf{4} \mathbf{b}^{\text {MOM }}$ at 298 K (grey lines, right) and time-resolved emission spectra showing the Ln-centered emissions ( $\mathrm{Tb}$ (green, $\lambda_{\mathrm{ex}}=335 \mathrm{~nm}, \lambda_{\mathrm{em}}=546 \mathrm{~nm}$ ), $\mathrm{Eu}\left(\mathrm{red}, \lambda_{\mathrm{ex}}=335 \mathrm{~nm}, \lambda_{\mathrm{em}}=615 \mathrm{~nm}\right), 298 \mathrm{~K}$, colored lines, right). 


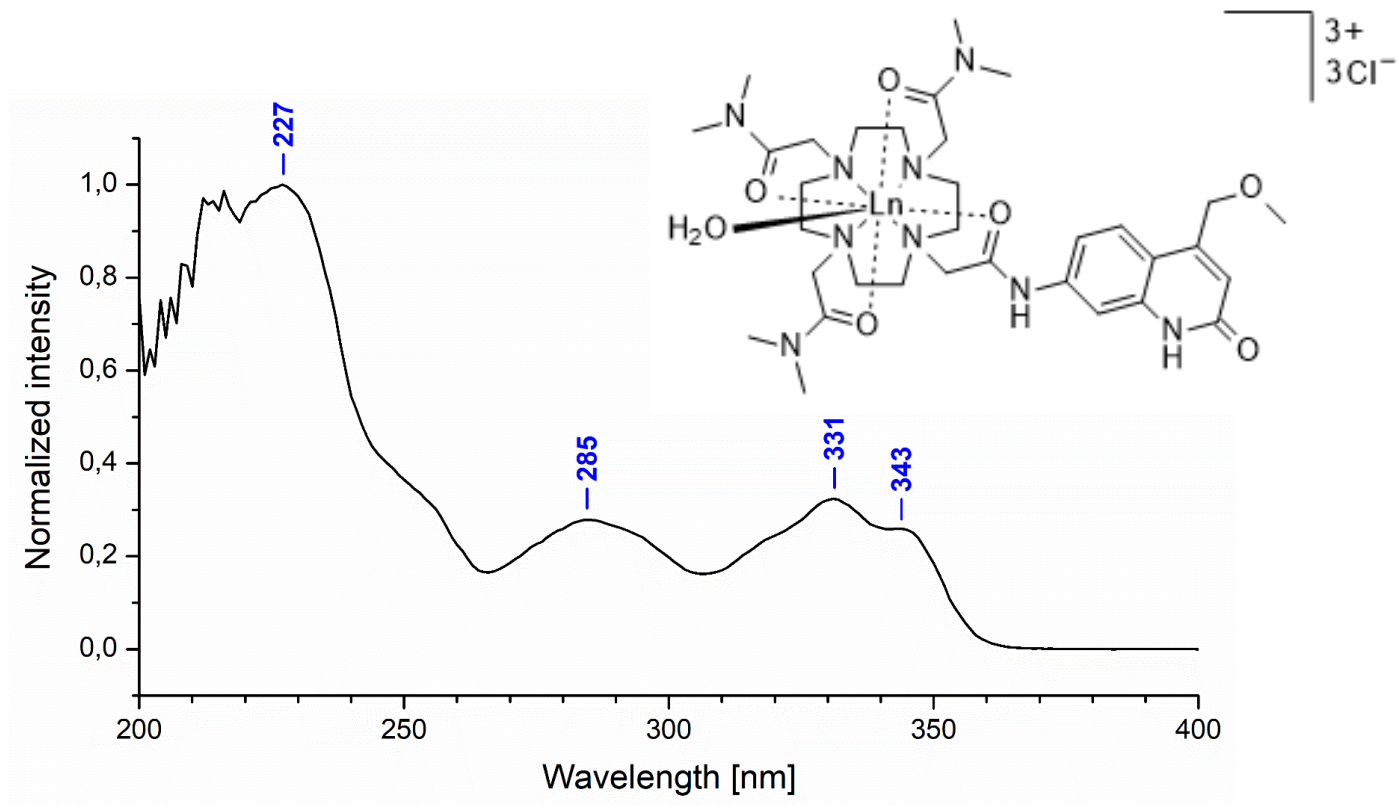

Figure S45. Normalized absorption spectrum of GdL4c $\mathbf{c}^{\text {MOM }}$ in 0.01 M PIPES-buffered aqueous solution, $\mathrm{pH}$ 6.5. Blue numbers show local maxima.

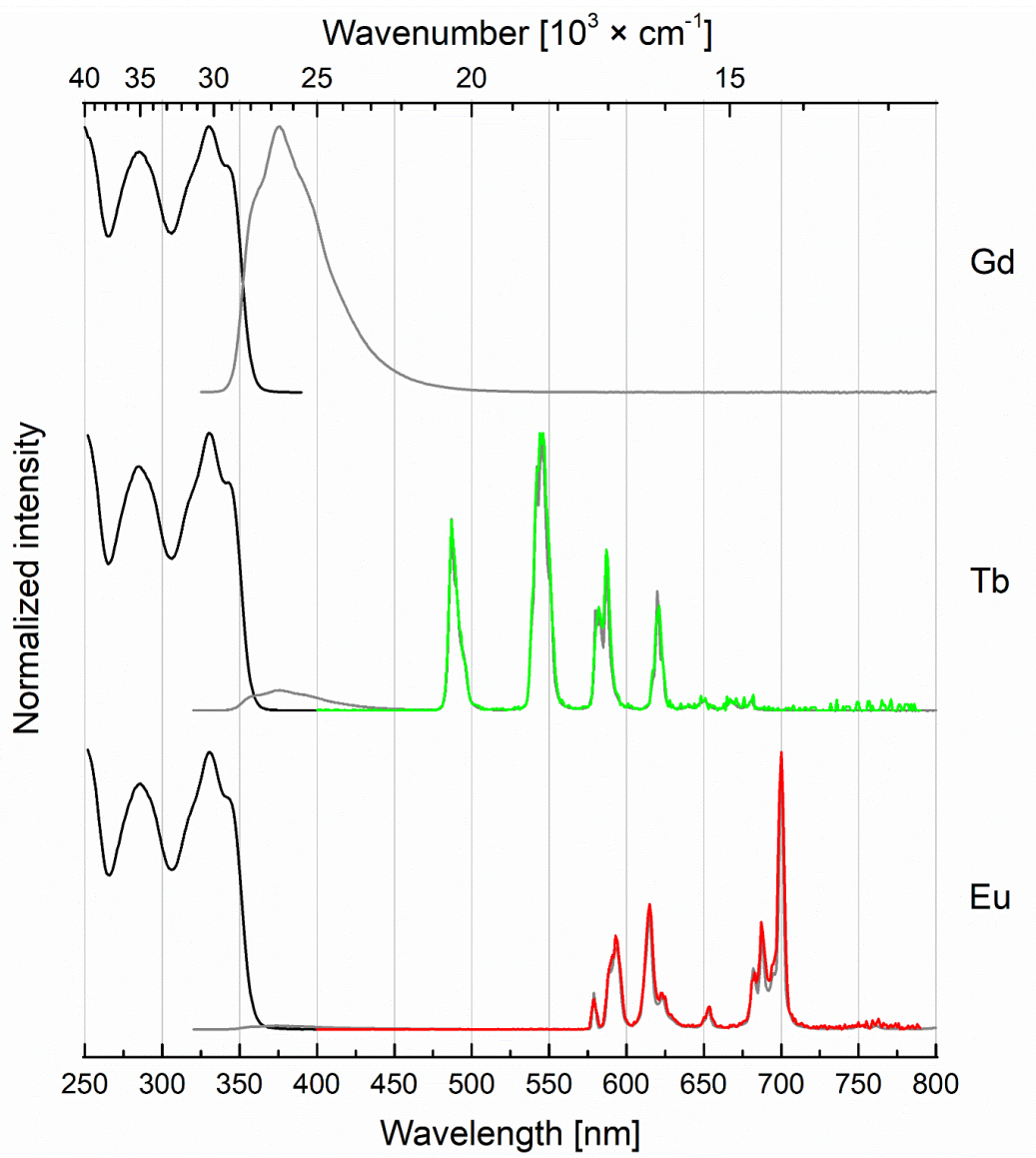

Figure S46. Excitation spectra of the Ln-centered emissions $\left(\mathrm{Tb}, \lambda_{\mathrm{em}}=545 \mathrm{~nm} ; \mathrm{Eu}, \lambda_{\mathrm{em}}=700\right.$ $\mathrm{nm}$ ) and of the ligand centered emission (GdL4 $\mathbf{c}^{\mathbf{M O M}}, \lambda_{\mathrm{em}}=405 \mathrm{~nm}$ ) at $298 \mathrm{~K}$ (black lines, left). Steady-state emission spectra of LnL4c $\mathbf{c}^{\text {MOM }}$ at 298 K (grey lines, right) and time-resolved emission spectra showing the Ln-centered emissions ( $\mathrm{Tb}$ (green, $\lambda_{\mathrm{ex}}=335 \mathrm{~nm}, \lambda_{\mathrm{em}}=546 \mathrm{~nm}$ ), $\mathrm{Eu}\left(\mathrm{red}, \lambda_{\mathrm{ex}}=335 \mathrm{~nm}, \lambda_{\mathrm{em}}=615 \mathrm{~nm}\right), 298 \mathrm{~K}$, colored lines, right). 


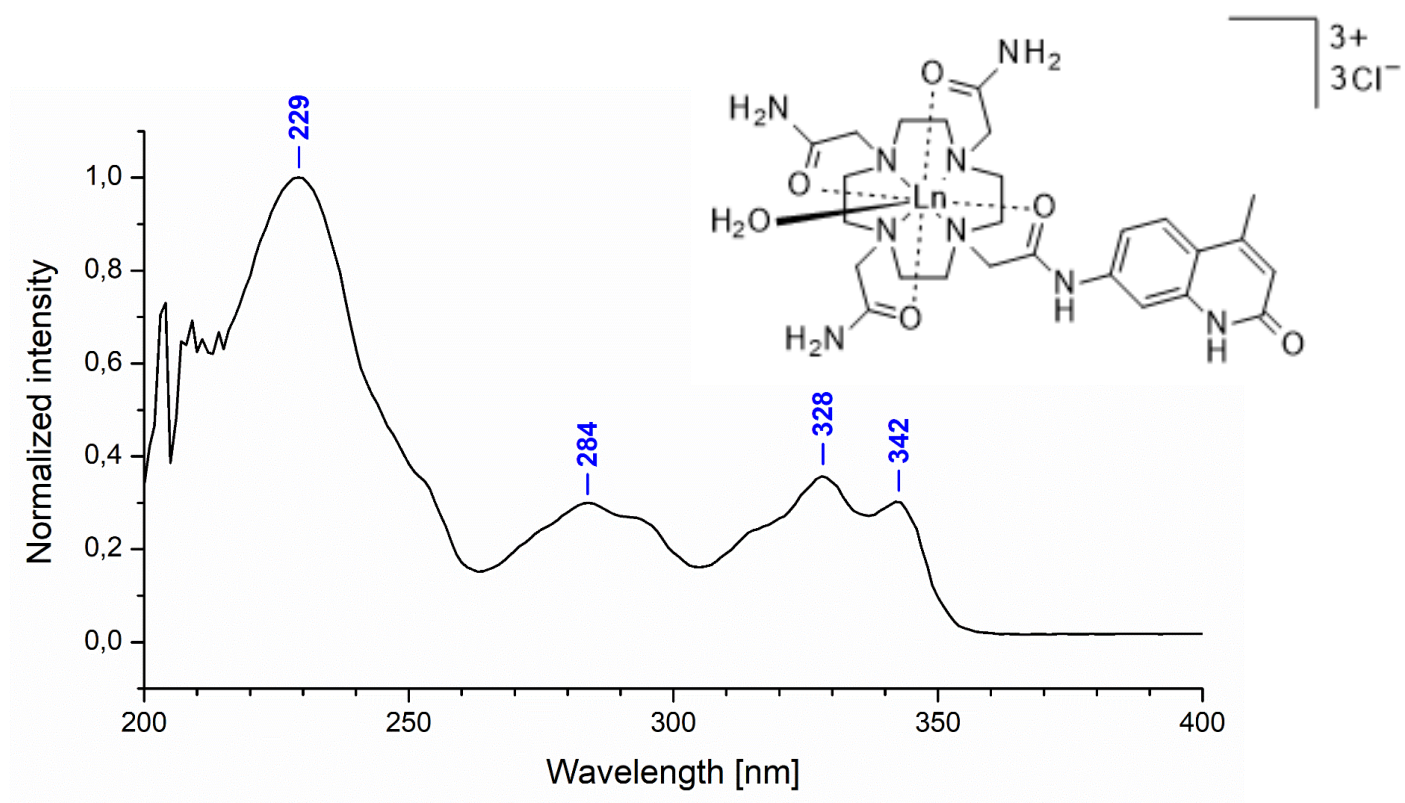

Figure S47. Normalized absorption spectrum of $\mathbf{G d L 4 a}^{\mathbf{M e}}$ in 0.01 M PIPES-buffered aqueous solution, pH 6.5. Blue numbers show local maxima.

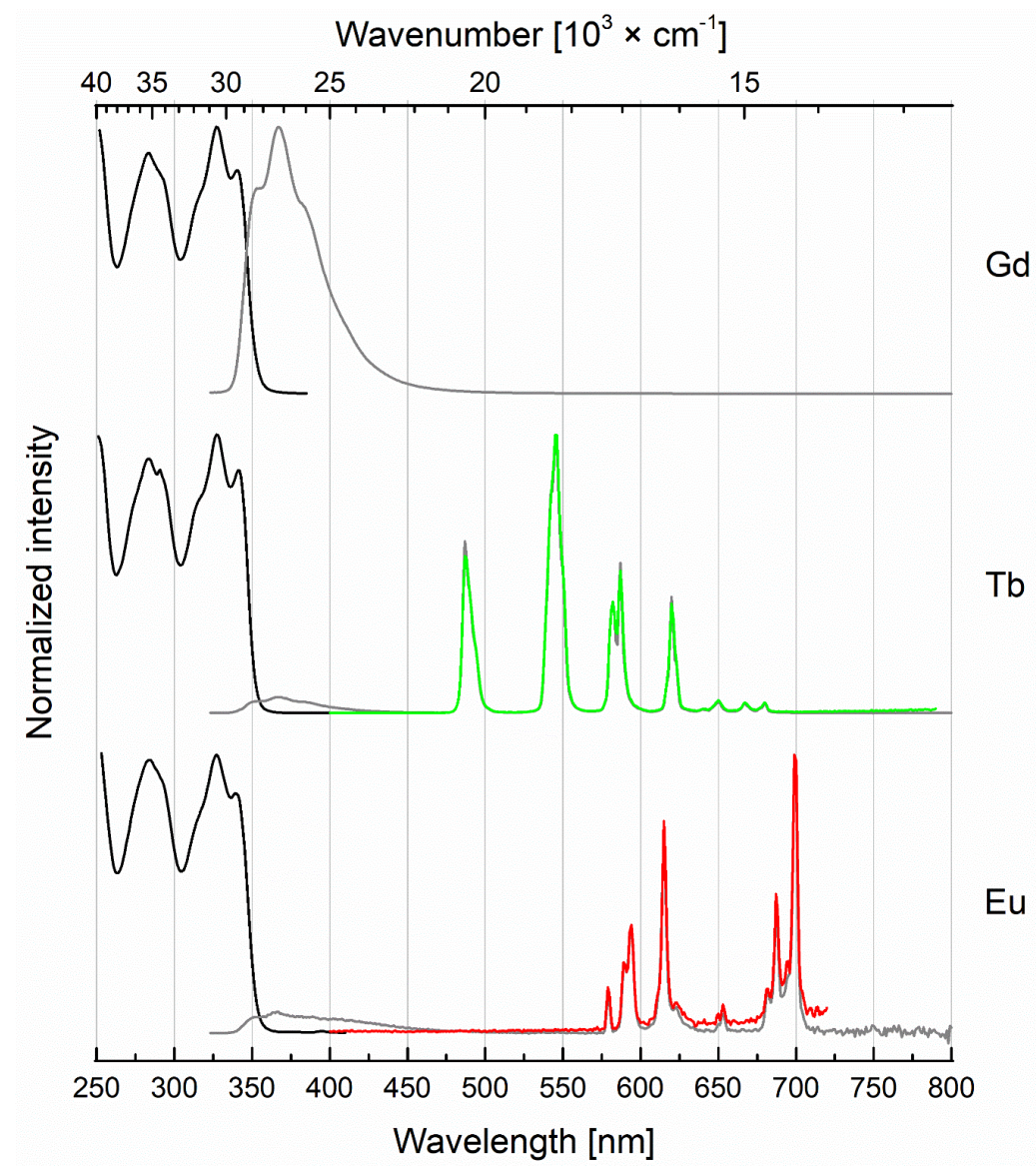

Figure S48. Excitation spectra of the Ln-centered emissions $\left(\mathrm{Tb}, \lambda_{\mathrm{em}}=545 \mathrm{~nm} ; \mathrm{Eu}, \lambda_{\mathrm{em}}=700\right.$ $\mathrm{nm})$ and of the ligand centered emission $\left(\mathbf{G d L 4 a}^{\mathbf{M e}}, \lambda_{\mathrm{em}}=400 \mathrm{~nm}\right)$ at $298 \mathrm{~K}$ (black lines, left). Steady-state emission spectra of $\mathbf{L n L 4 \mathbf { a } ^ { \mathrm { Me } }}$ at $298 \mathrm{~K}$ (grey lines, right) and time-resolved emission spectra showing the Ln-centered emissions ( $\mathrm{Tb}$ (green, $\lambda_{\mathrm{ex}}=328 \mathrm{~nm}, \lambda_{\mathrm{em}}=545 \mathrm{~nm}$ ), $\mathrm{Eu}\left(\mathrm{red}, \lambda_{\mathrm{ex}}=328 \mathrm{~nm}, \lambda_{\mathrm{em}}=700 \mathrm{~nm}\right), 298 \mathrm{~K}$, colored lines, right). 


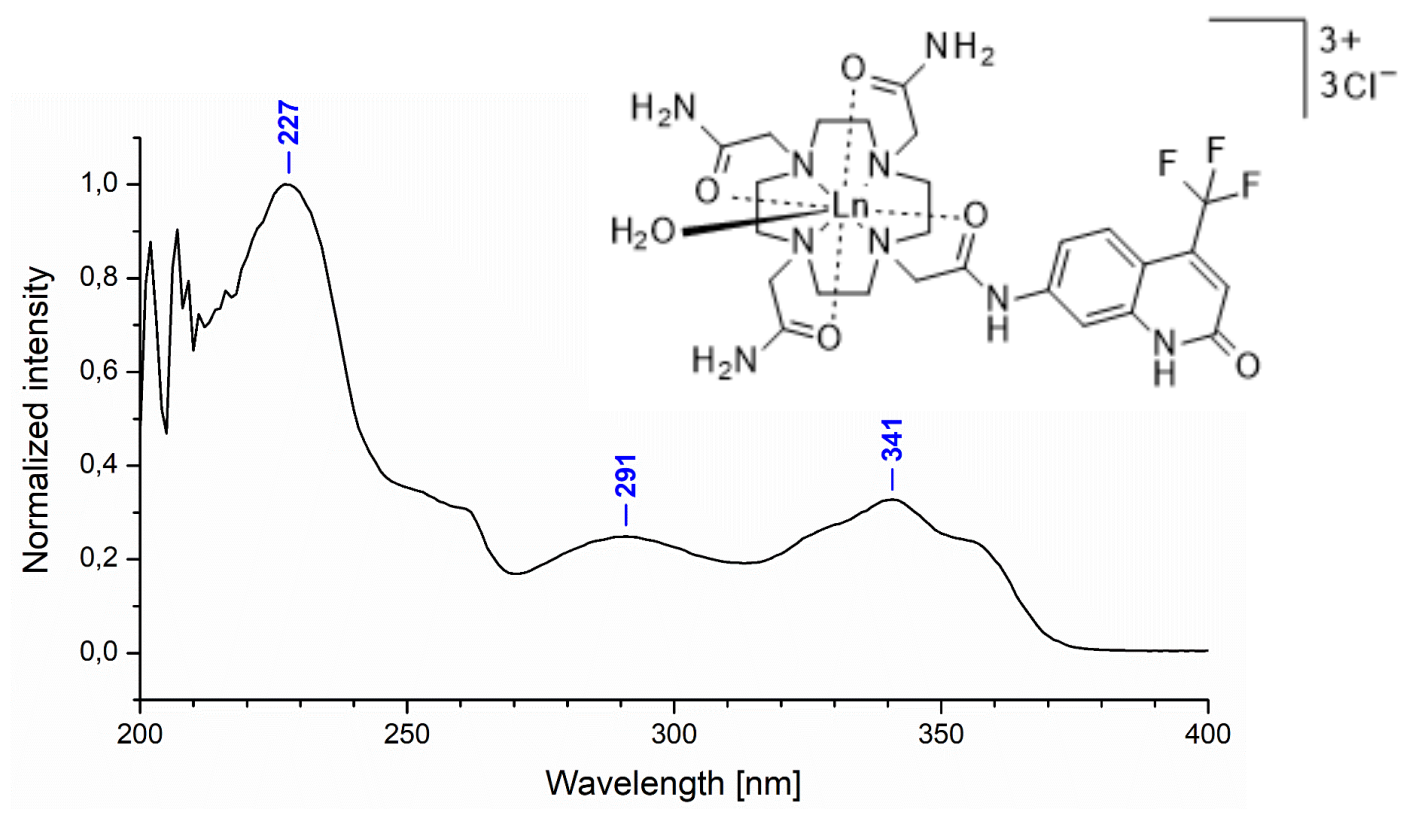

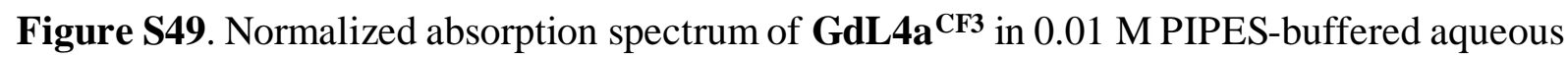
solution, pH 6.5. Blue numbers show local maxima.

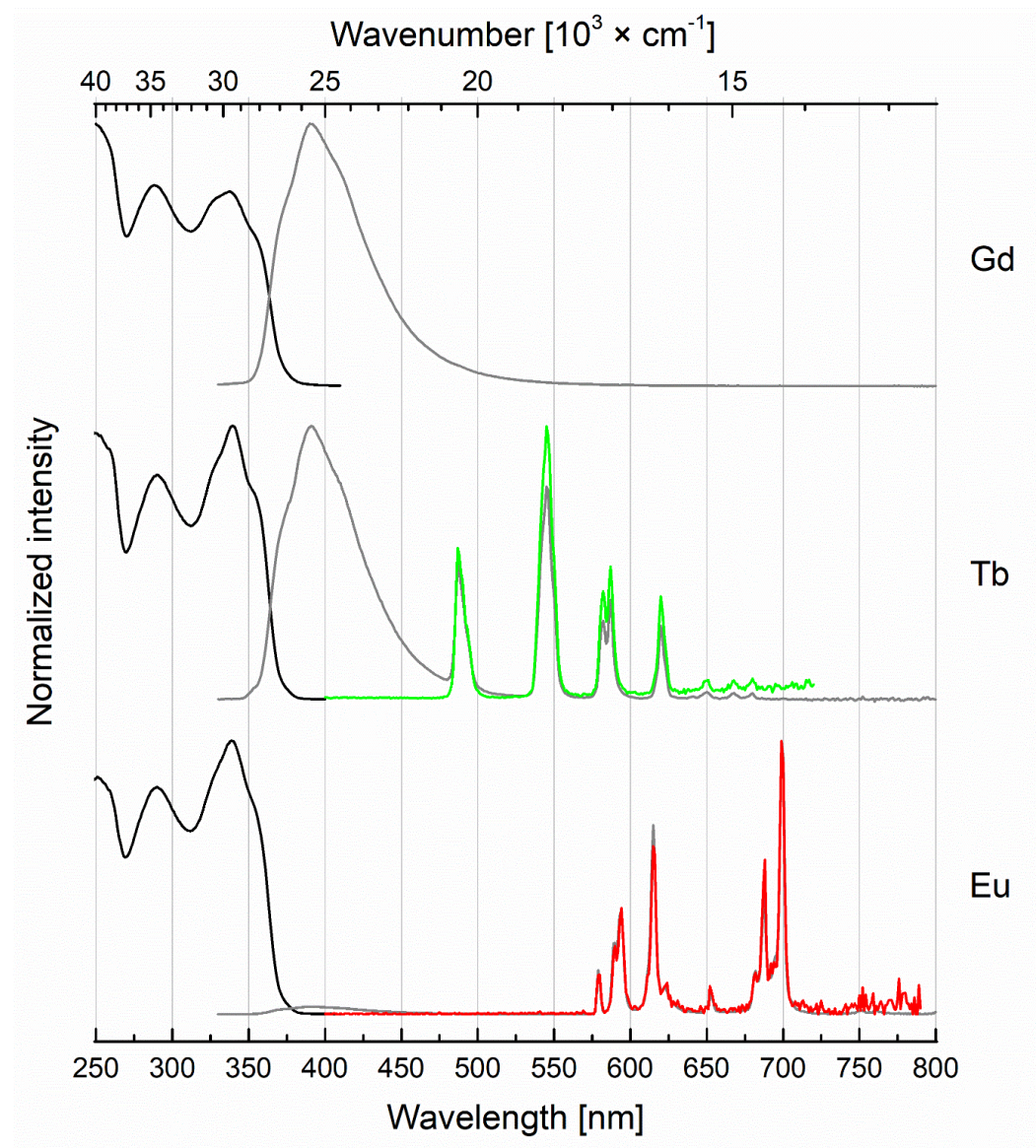

Figure S50. Excitation spectra of the Ln-centered emissions $\left(\mathrm{Tb}, \lambda_{\mathrm{em}}=545 \mathrm{~nm}\right.$; Eu, $\lambda_{\mathrm{em}}=700$

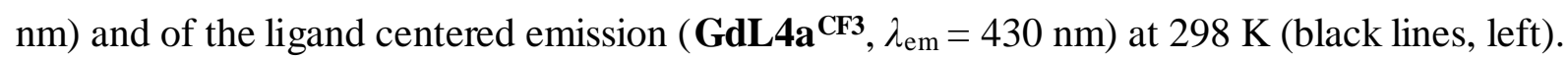

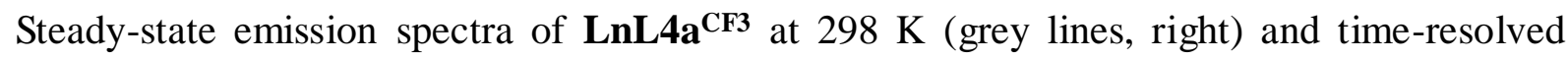
emission spectra showing the Ln-centered emissions ( $\mathrm{Tb}$ (green, $\lambda_{\mathrm{ex}}=340 \mathrm{~nm}, \lambda_{\mathrm{em}}=546 \mathrm{~nm}$ ), $\mathrm{Eu}\left(\mathrm{red}, \lambda_{\mathrm{ex}}=340 \mathrm{~nm}, \lambda_{\mathrm{em}}=616 \mathrm{~nm}\right), 298 \mathrm{~K}$, colored lines, right). 
Table S18. Fluorescence lifetimes of $\mathbf{G d L}$ and $\mathbf{T b L}$ from two independent measurements $\left(\lambda_{\mathrm{em}}\right.$ $=390 \mathrm{~nm}$, PIPES buffer, nominally $10 \mu \mathrm{M}$ concentrations).

\begin{tabular}{|c|c|c|}
\hline Complex & $\boldsymbol{\tau}_{f, L}[n s]^{\mathrm{a}}$ & Standard deviation [\%] \\
\hline \multirow{2}{*}{ GdL1 ${ }^{M O M}$} & 0.427 & 0.332 \\
\hline & 0.425 & \\
\hline \multirow{2}{*}{ GdL2 2MOM } & 0.416 & 0.845 \\
\hline & 0.421 & \\
\hline \multirow{2}{*}{ GdL3 $^{\text {MOM }}$} & 0.411 & 0.514 \\
\hline & 0.414 & \\
\hline \multirow{2}{*}{ GdL4a $\mathbf{a}^{\text {MOM }}$} & 0.416 & 2.25 \\
\hline & 0.403 & \\
\hline \multirow{2}{*}{ GdL4b } & 0.410 & 1.57 \\
\hline & 0.401 & \\
\hline \multirow{2}{*}{ GdL4c } & 0.411 & 0.865 \\
\hline & 0.406 & \\
\hline \multirow{2}{*}{ TbL1 ${ }^{\text {MOM }}$} & 0.347 & 0.609 \\
\hline & 0.350 & \\
\hline \multirow{2}{*}{ TbL2 $2^{\text {MOM }}$} & 0.326 & 2.56 \\
\hline & 0.338 & \\
\hline \multirow{2}{*}{ TbL3 $3^{\text {MOM }}$} & 0.319 & 1.32 \\
\hline & 0.325 & \\
\hline \multirow{2}{*}{ TbL4a ${ }^{\text {MOM }}$} & 0.308 & 3.80 \\
\hline & 0.325 & \\
\hline \multirow{2}{*}{ TbL4b } & 0.319 & 3.67 \\
\hline & 0.336 & \\
\hline \multirow{2}{*}{ TbL4c $\mathrm{c}^{\mathrm{MOM}}$} & 0.319 & 3.25 \\
\hline & 0.334 & \\
\hline
\end{tabular}

${ }^{a}$ Fluorescence lifetime values from two independent measurements.

Table S19. The fluorescence lifetimes of $\mathbf{L 1}^{\text {MOM }}\left(\lambda_{\mathrm{em}}=390 \mathrm{~nm}\right.$, PIPES buffer, nominally 10 $\mu \mathrm{M}$ concentrations).

\begin{tabular}{lc}
\hline Compound & $\boldsymbol{\tau}_{\boldsymbol{f}, \boldsymbol{L}}[\boldsymbol{n s}]^{a}$ \\
\hline $\mathbf{L 1}^{\mathrm{MOM}}(\mathrm{pH}=3.70)$ & $0.495 \pm 0.001$ \\
$\mathbf{L 1}^{\mathrm{MOM}}(\mathrm{pH}=7.75)$ & $0.486 \pm 0.002$ \\
\hline
\end{tabular}

${ }^{a}$ Fluorescence lifetime given with the standard deviation of the fit. 
Table S20. Comparison of standards lifetimes with literature values.

\begin{tabular}{lccc}
\hline Compound & $\tau[n s]^{\mathrm{a}}$ & $\lambda_{\text {ex }}[\mathrm{nm}]^{\mathrm{b}}$ & $\tau_{\text {ref }}[n s]^{\mathrm{c}}$ \\
\hline Rose Bengal & $0.525 \pm 0.003$ & 577 & $0.543 \pm 0.009^{8}$ \\
Erythrosin B & $0.453 \pm 0.003$ & 554 & $0.47 \pm 0.02^{9}$ \\
Erythrosin B (95+\%, spirit soluble) & $0.462 \pm 0.003$ & 556 & $0.47 \pm 0.022^{9}$ \\
\hline
\end{tabular}

${ }^{a}$ Fluorescence lifetime given with the standard deviation of the fit, ${ }^{b}$ emission wavelength of the experiment, ${ }^{\mathrm{c}}$ literature values for Rose $\mathrm{Bengal}^{8}$ and Erythrosin $\mathrm{B}^{9}$ in methanol.

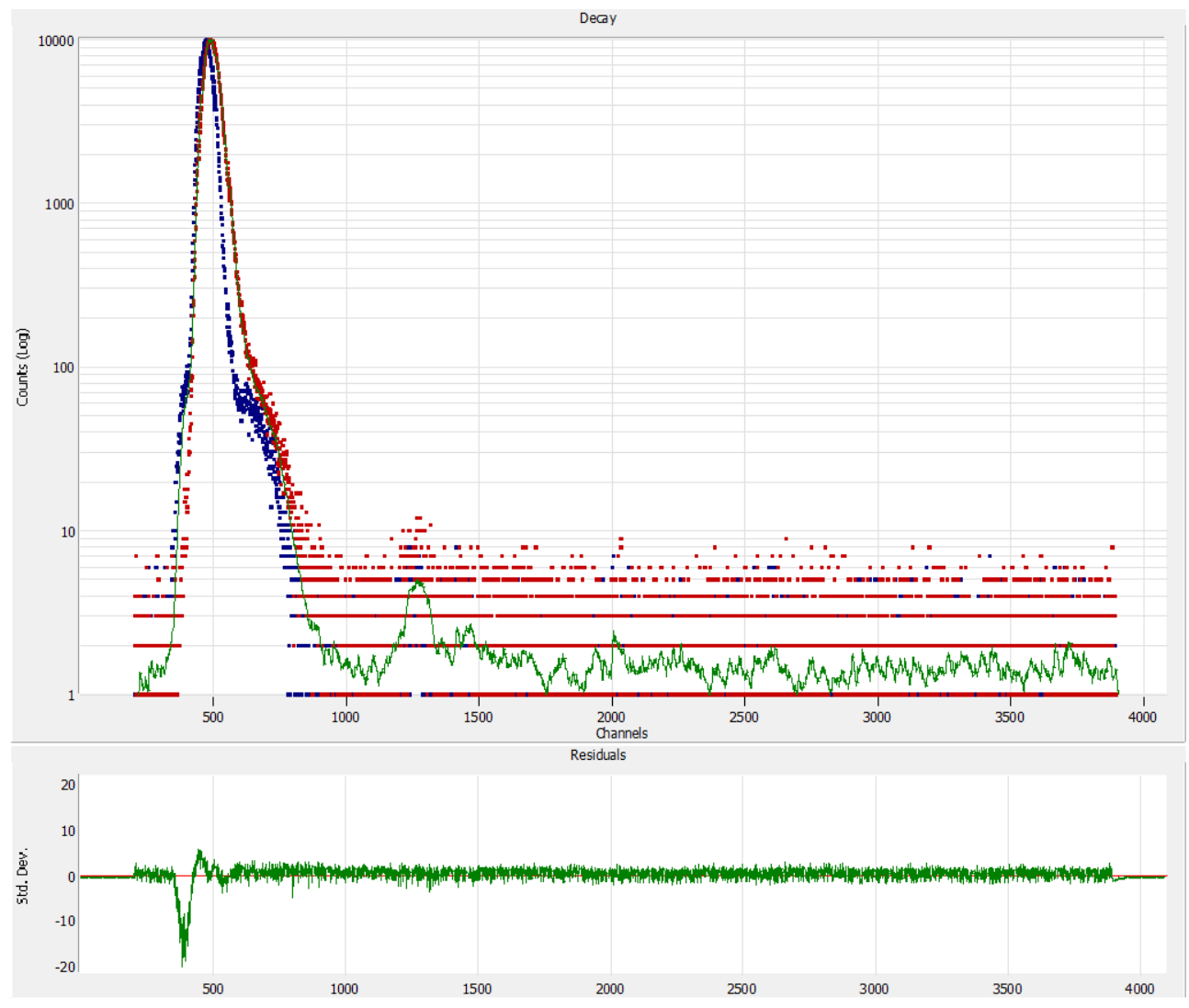

Figure S51. The fluorescence decay fit of Rose Bengal $\left(\lambda_{\mathrm{em}}=577 \mathrm{~nm}, \mathrm{MeOH}\right)$ and residuals of the fit. 


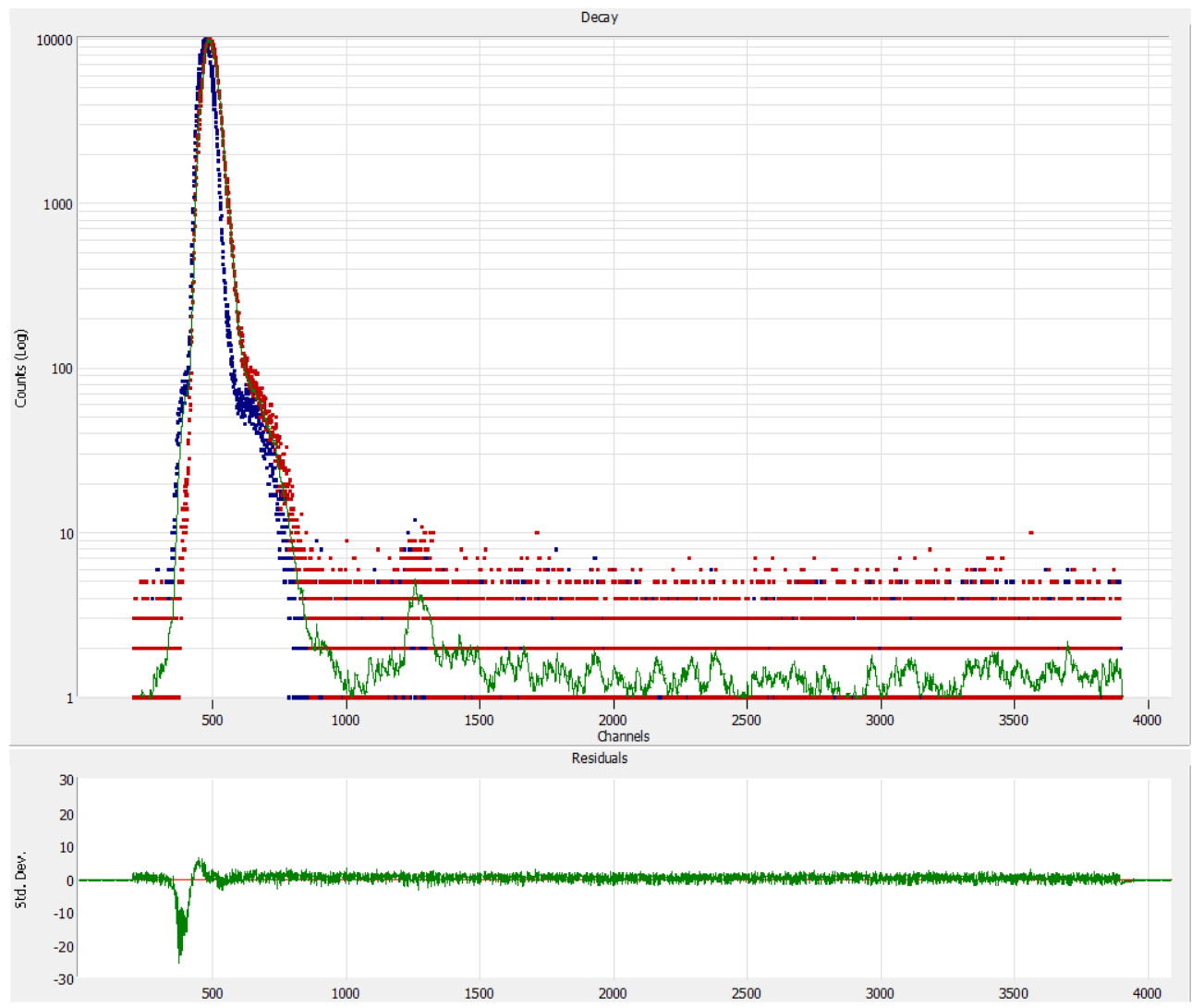

Figure S52. The fluorescence decay fit of Erythrosin $\mathrm{B}\left(\lambda_{\mathrm{em}}=554 \mathrm{~nm}, \mathrm{MeOH}\right)$ and residuals of the fit.
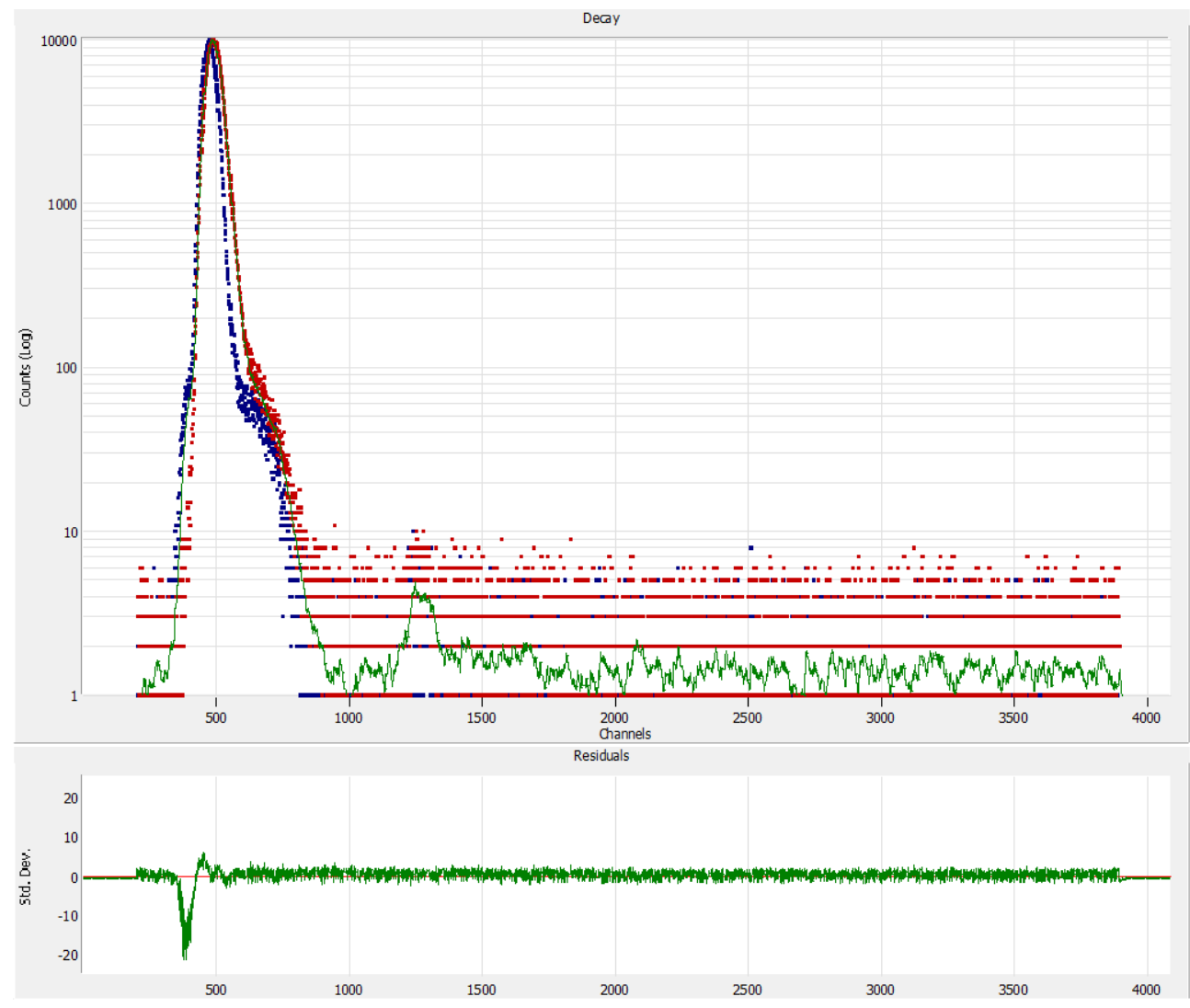

Figure S53. The fluorescence decay fit of Erythrosin B $(95+\%$, spirit soluble $)\left(\lambda_{\mathrm{em}}=556 \mathrm{~nm}\right.$, $\mathrm{MeOH}$ ) and residuals of the fit. 


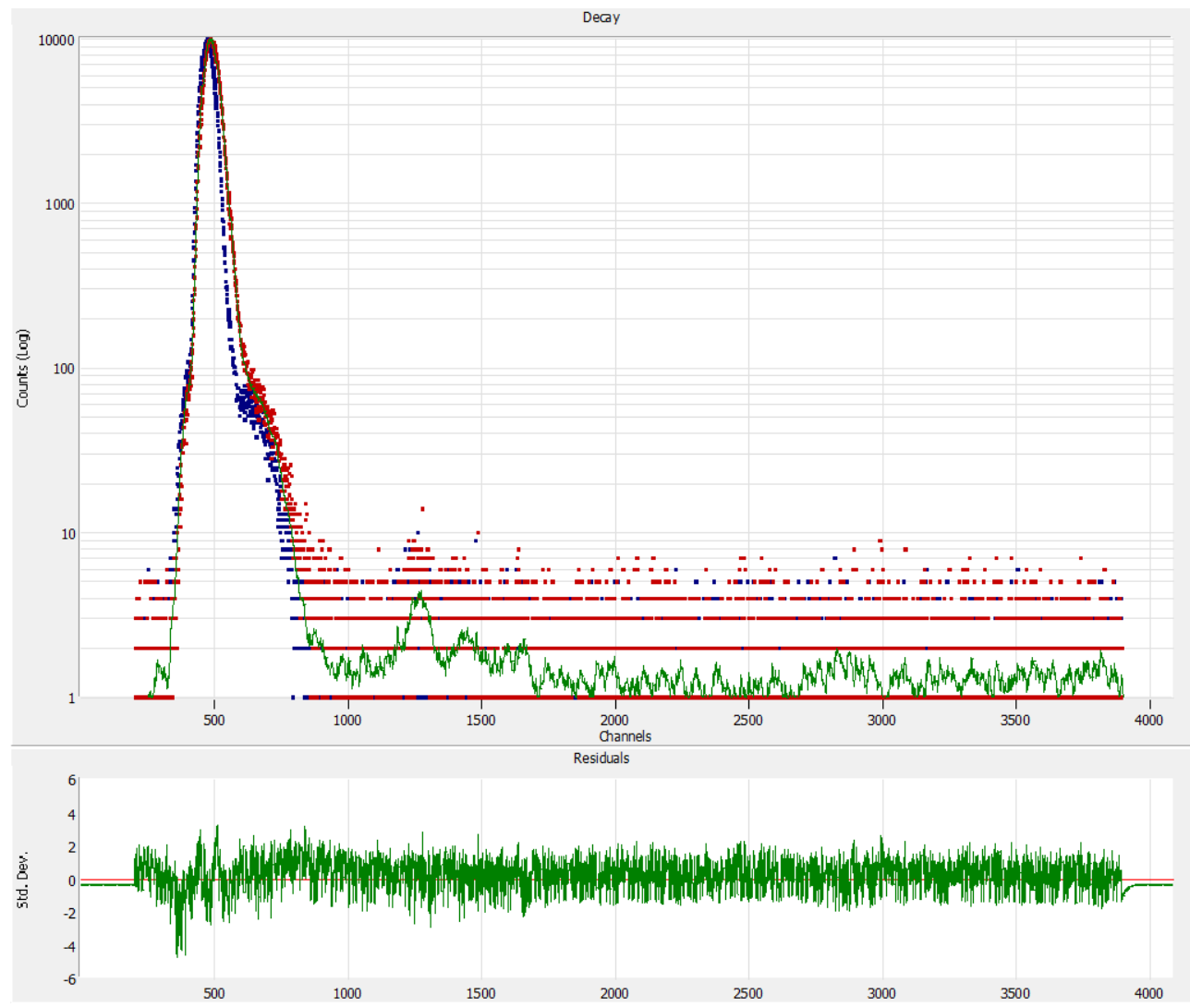

Figure S54. The fluorescence decay fit of $\operatorname{GdL1}^{\text {MOM }}\left(\lambda_{\mathrm{em}}=390 \mathrm{~nm}\right.$, PIPES buffer $)$ and residuals of the fit.

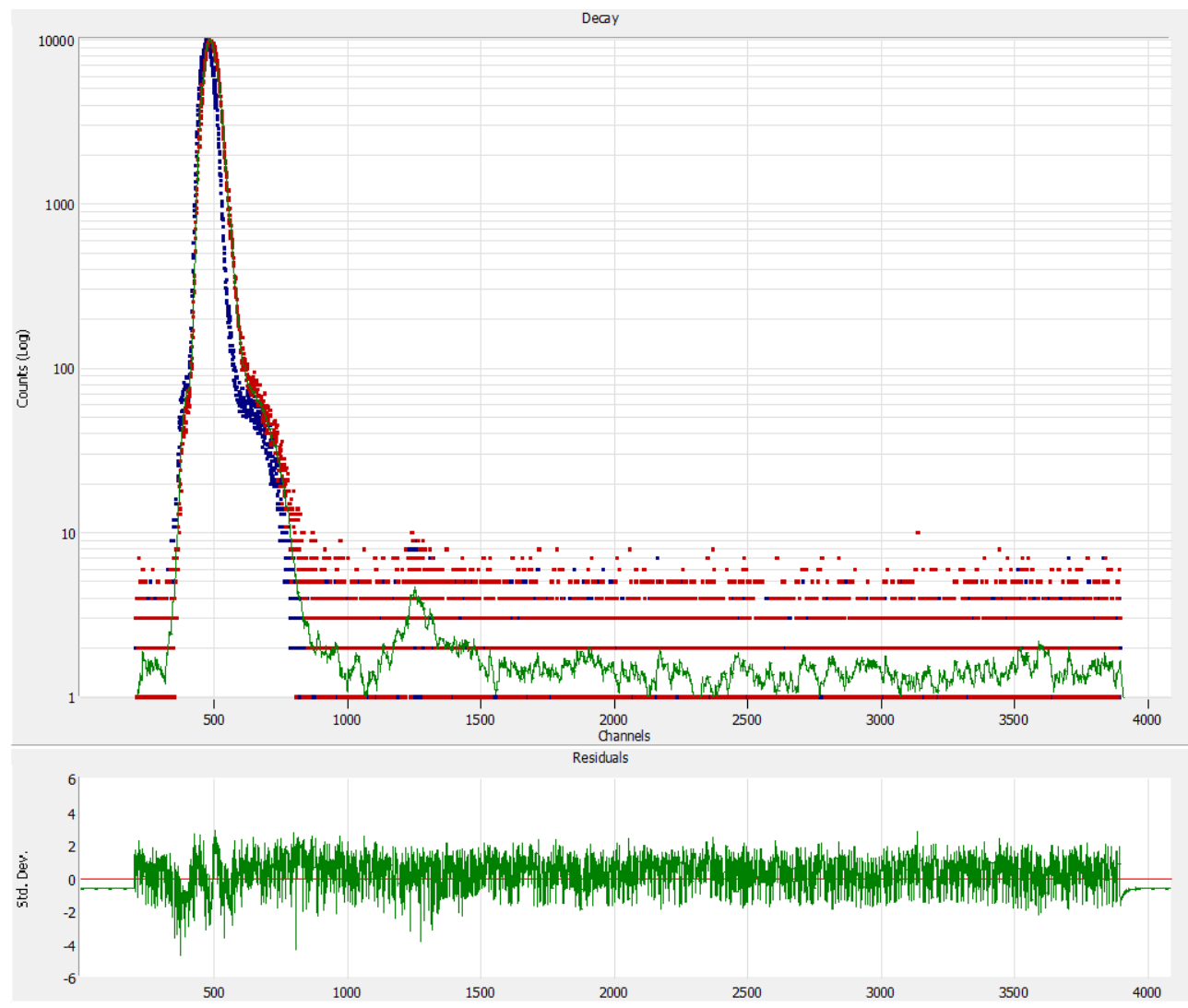

Figure S55. The fluorescence decay fit of GdL2 ${ }^{\text {MOM }}\left(\lambda_{\mathrm{em}}=390 \mathrm{~nm}\right.$, PIPES buffer $)$ and residuals of the fit. 

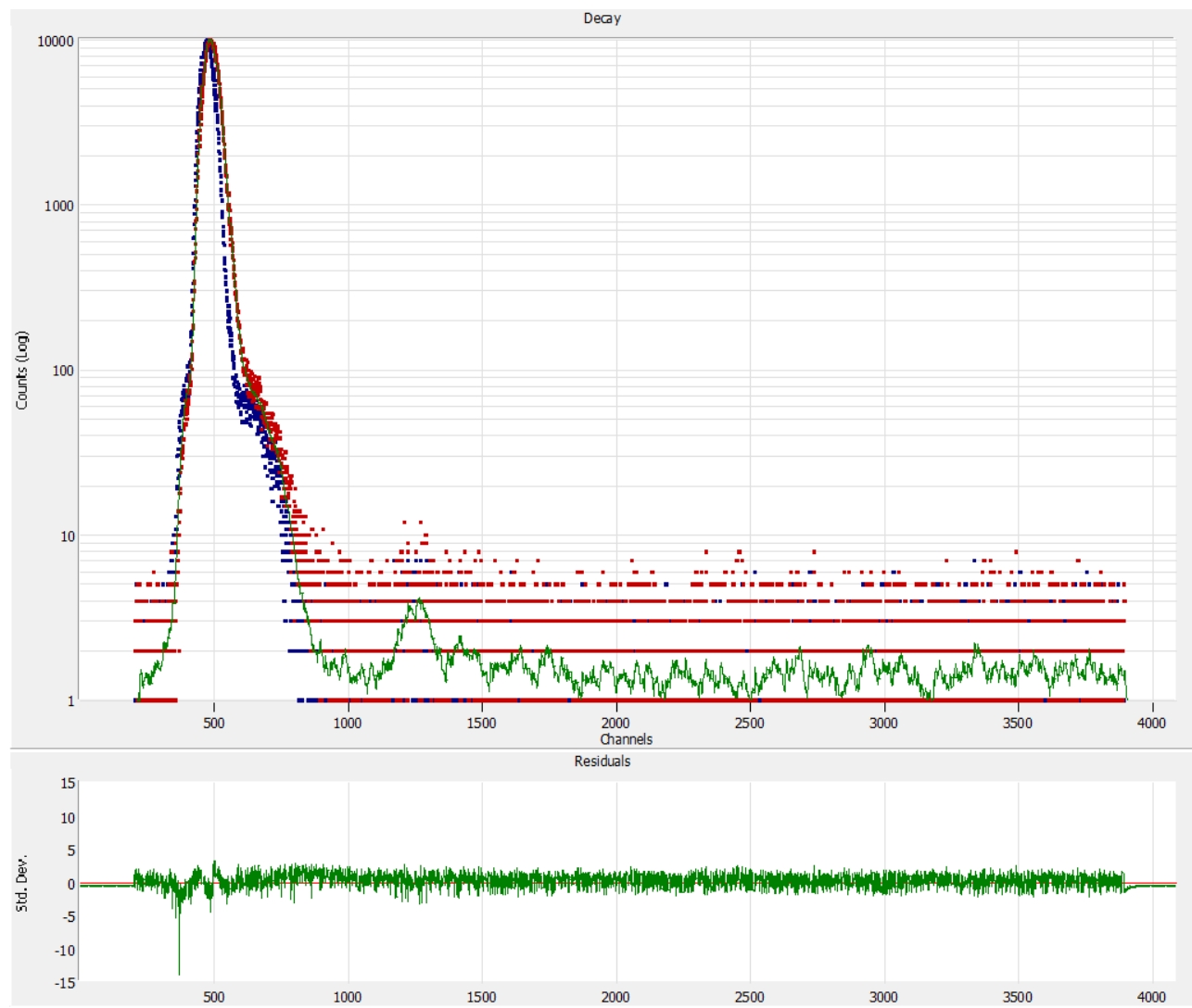

Figure S56. The fluorescence decay fit of $\mathbf{G d L 3}^{\mathbf{M O M}}\left(\lambda_{\mathrm{em}}=390 \mathrm{~nm}\right.$, PIPES buffer $)$ and residuals of the fit.

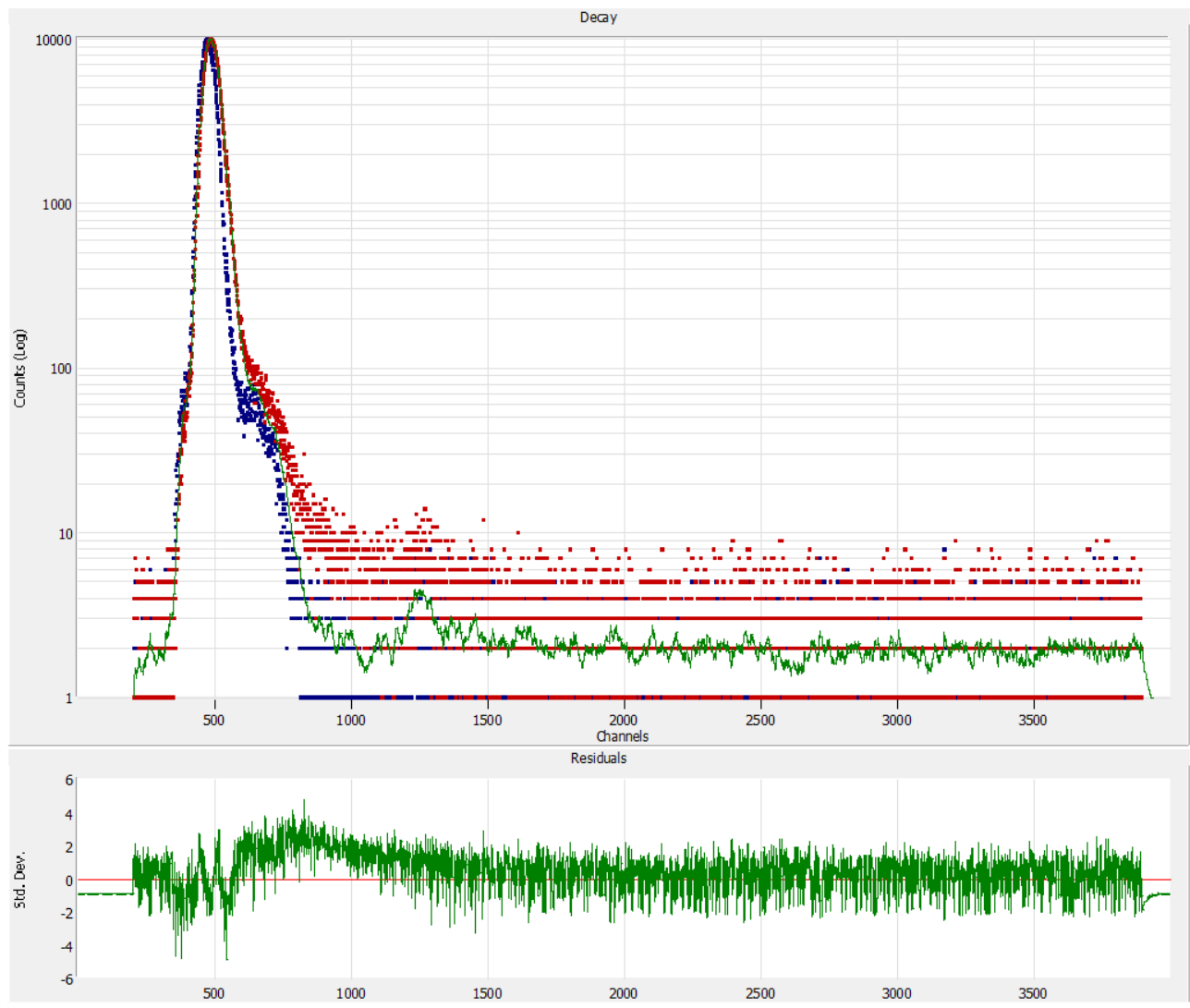

Figure S57. The fluorescence decay fit of GdL4a $\mathbf{a}^{\text {MOM }}\left(\lambda_{\mathrm{em}}=390 \mathrm{~nm}\right.$, PIPES buffer) and residuals of the fit. 


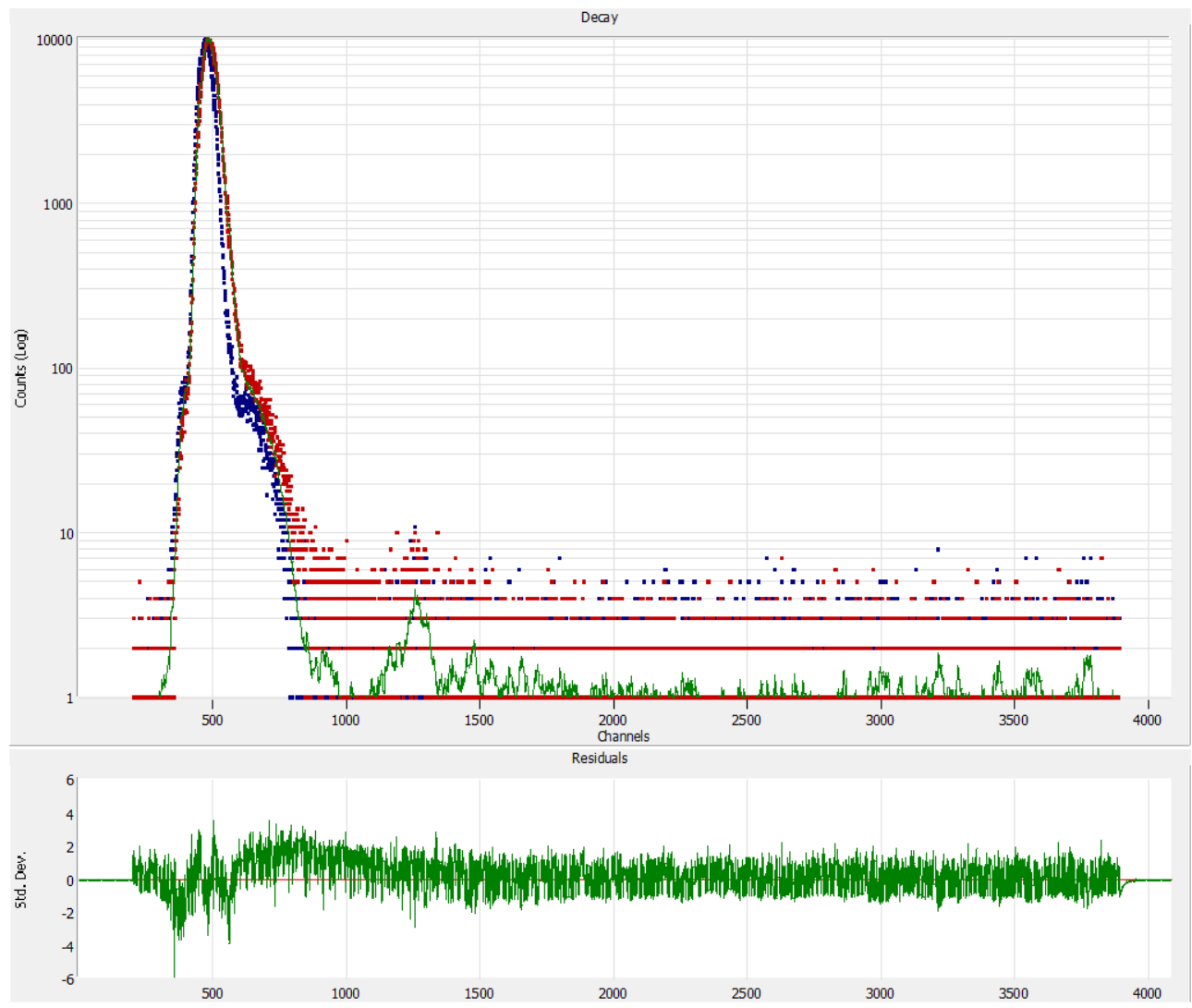

Figure S58. The fluorescence decay fit of GdL4b $\mathbf{b}^{\mathbf{M O M}}\left(\lambda_{\mathrm{em}}=390 \mathrm{~nm}\right.$, PIPES buffer $)$ and residuals of the fit.

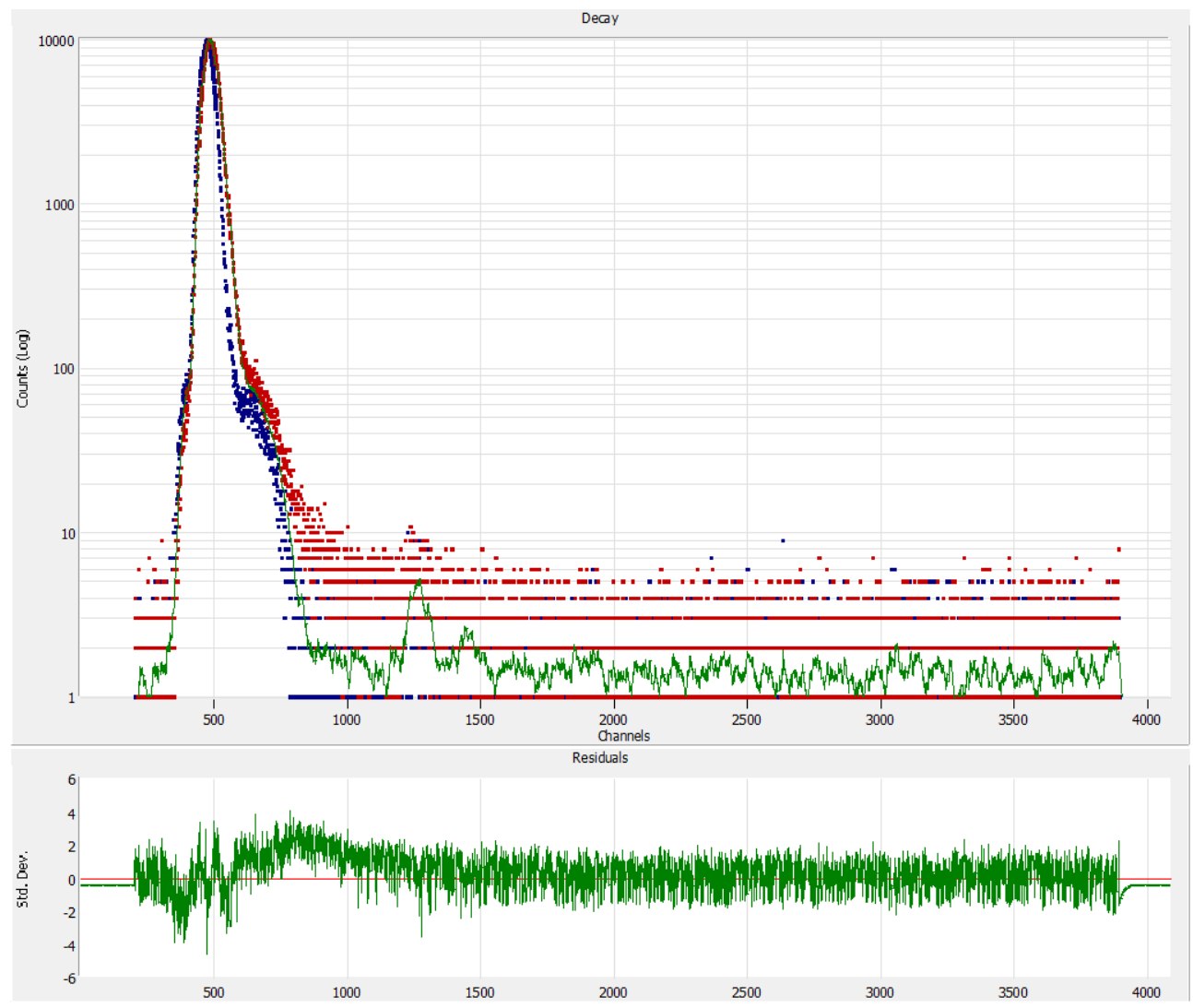

Figure S59. The fluorescence decay fit of GdL4c $\mathbf{c}^{\text {MOM }}\left(\lambda_{\mathrm{em}}=390 \mathrm{~nm}\right.$, PIPES buffer) and residuals of the fit. 


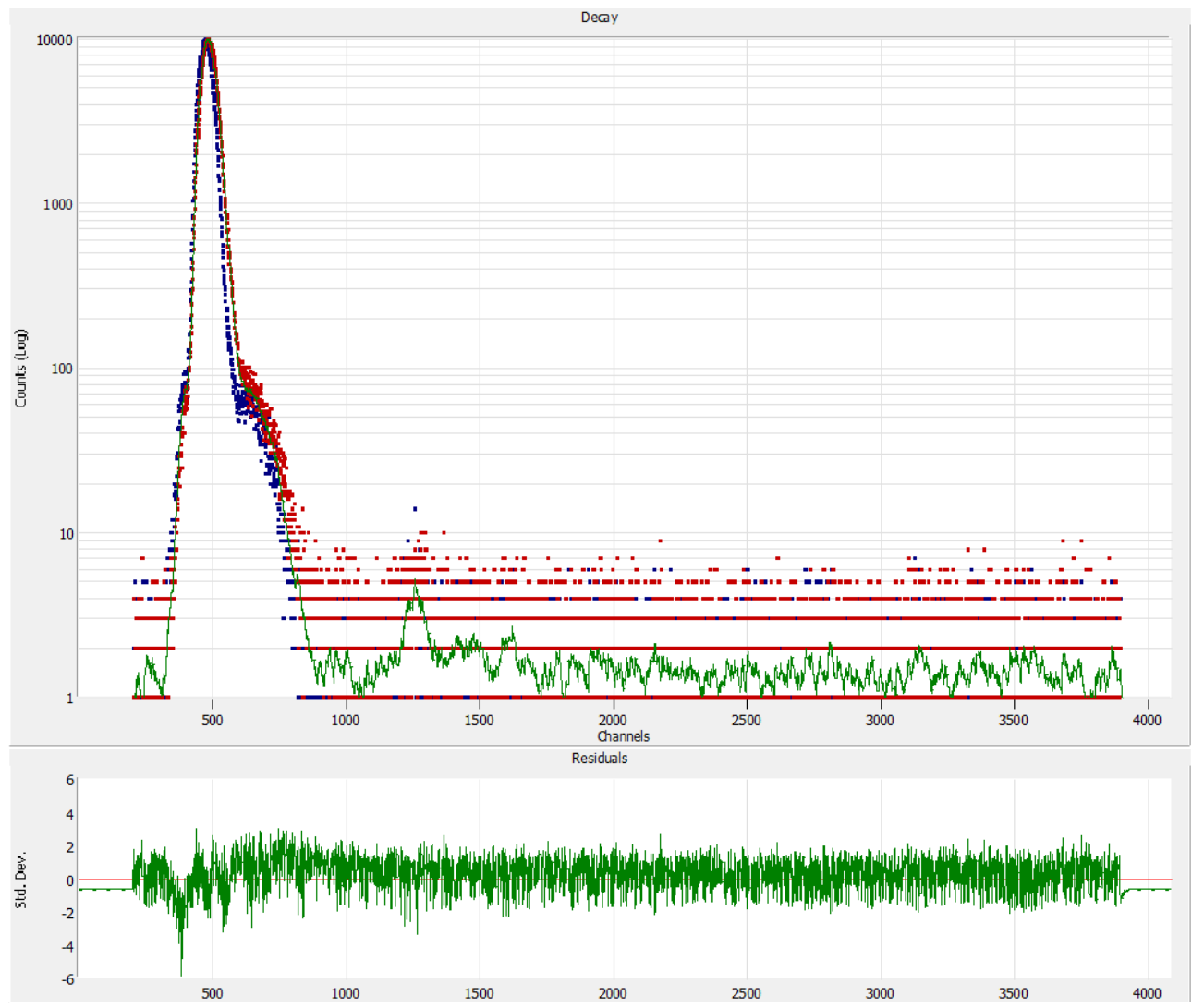

Figure S60. The fluorescence decay fit of TbL1 MOM $\left(\lambda_{\mathrm{em}}=390 \mathrm{~nm}\right.$, PIPES buffer) and residuals of the fit.

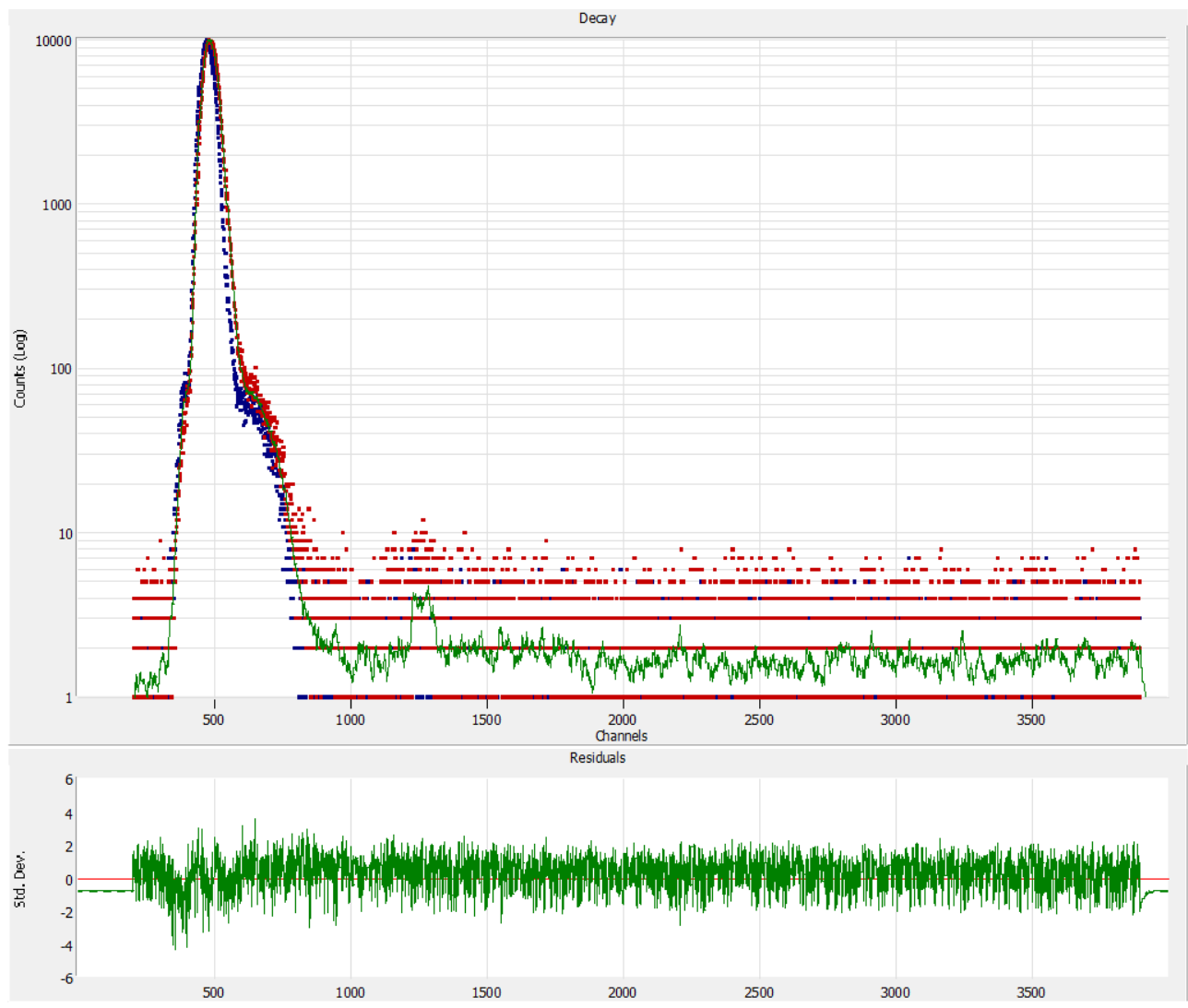

Figure S61. The fluorescence decay fit of TbL2 $\mathbf{2}^{\text {MOM }}\left(\lambda_{\mathrm{em}}=390 \mathrm{~nm}\right.$, PIPES buffer) and residuals of the fit. 


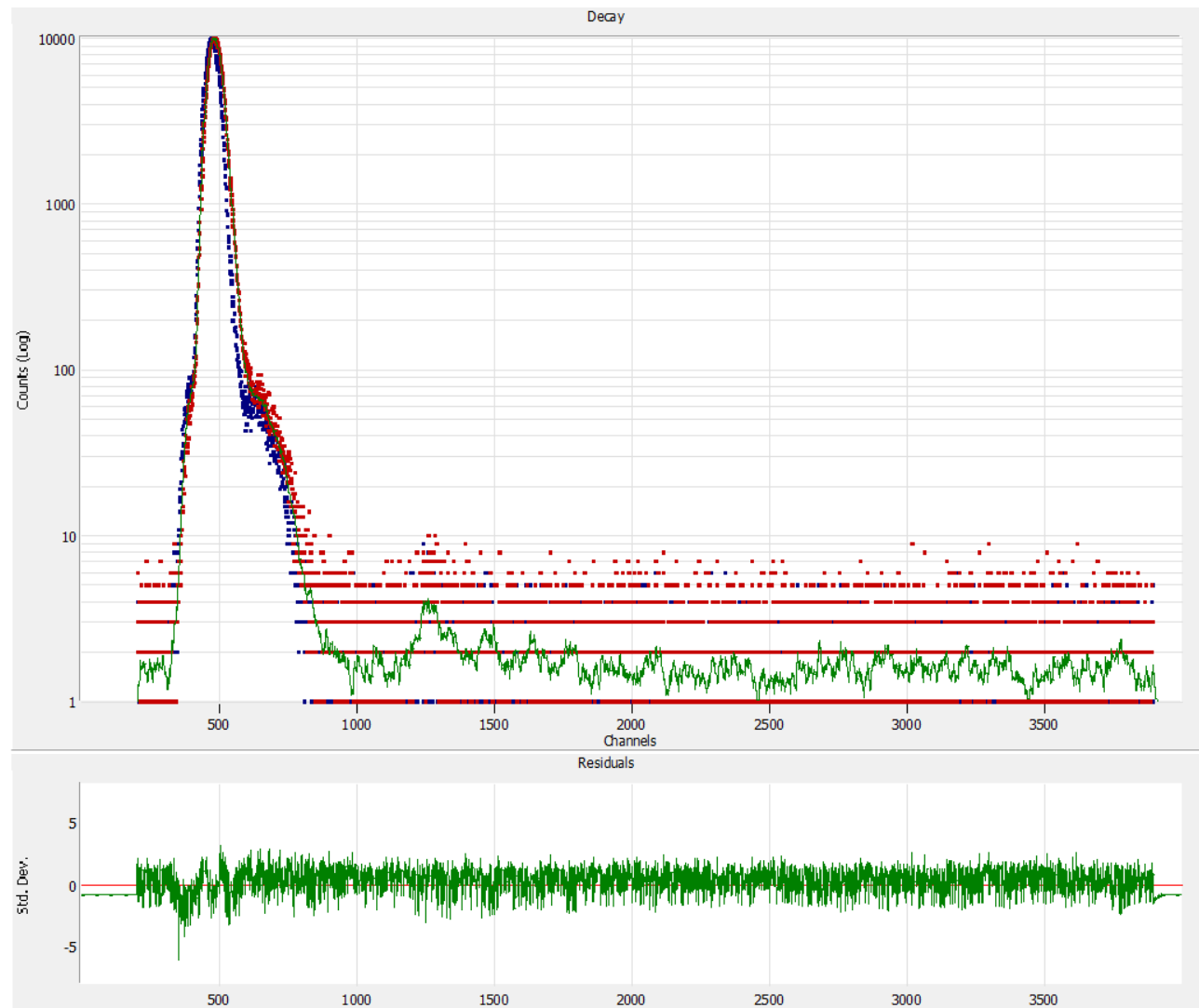

Figure S62. The fluorescence decay fit of TbL3 ${ }^{\text {MOM }}\left(\lambda_{\mathrm{em}}=390 \mathrm{~nm}\right.$, PIPES buffer $)$ and residuals of the fit.

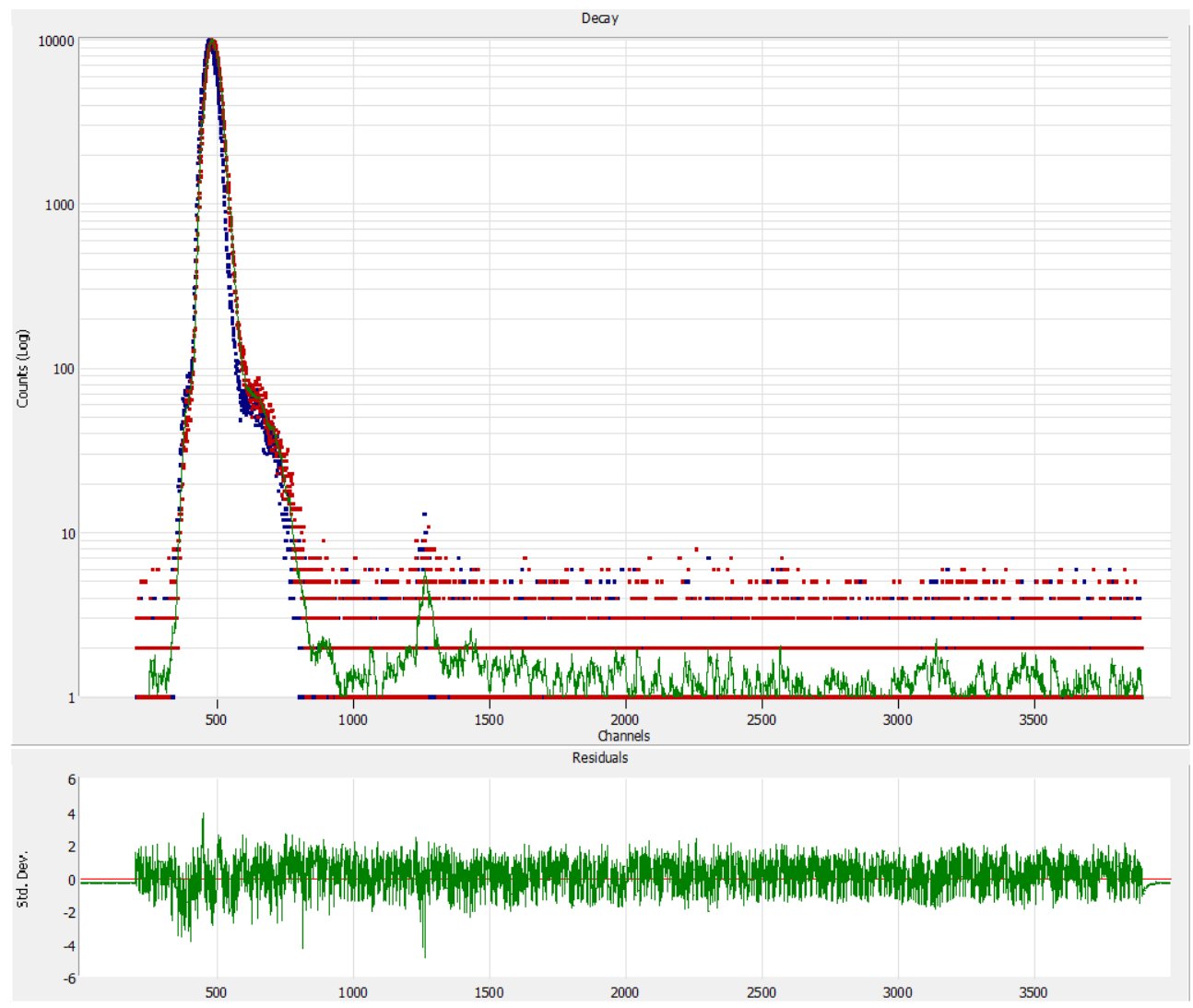

Figure S63. The fluorescence decay fit of TbL4a $\mathbf{A}^{\text {MOM }}\left(\lambda_{\mathrm{em}}=390 \mathrm{~nm}\right.$, PIPES buffer $)$ and residuals of the fit. 

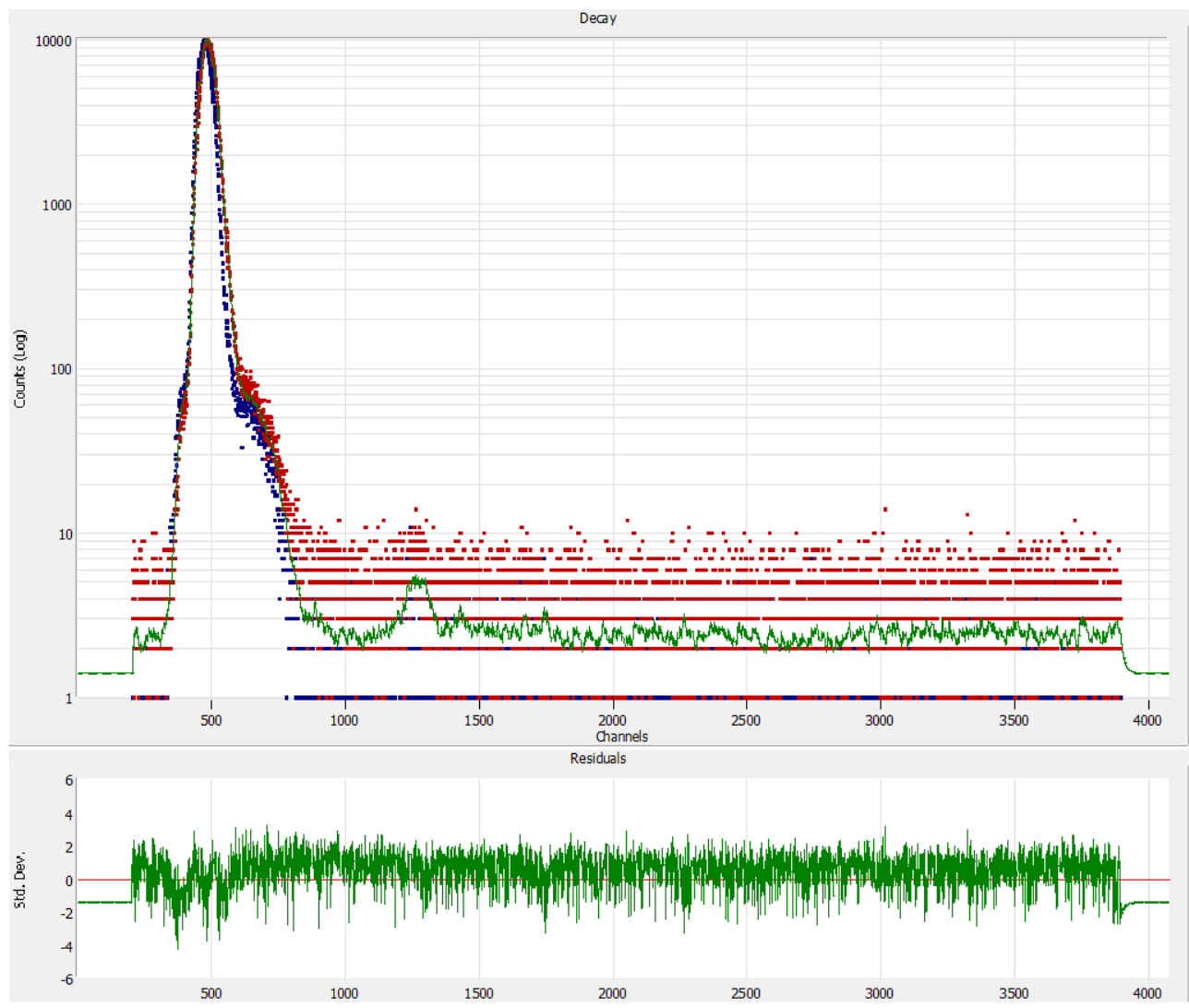

Figure S64. The fluorescence decay fit of TbL4b $\mathbf{b}^{\text {MOM }}\left(\lambda_{\mathrm{em}}=390 \mathrm{~nm}\right.$, PIPES buffer $)$ and residuals of the fit.

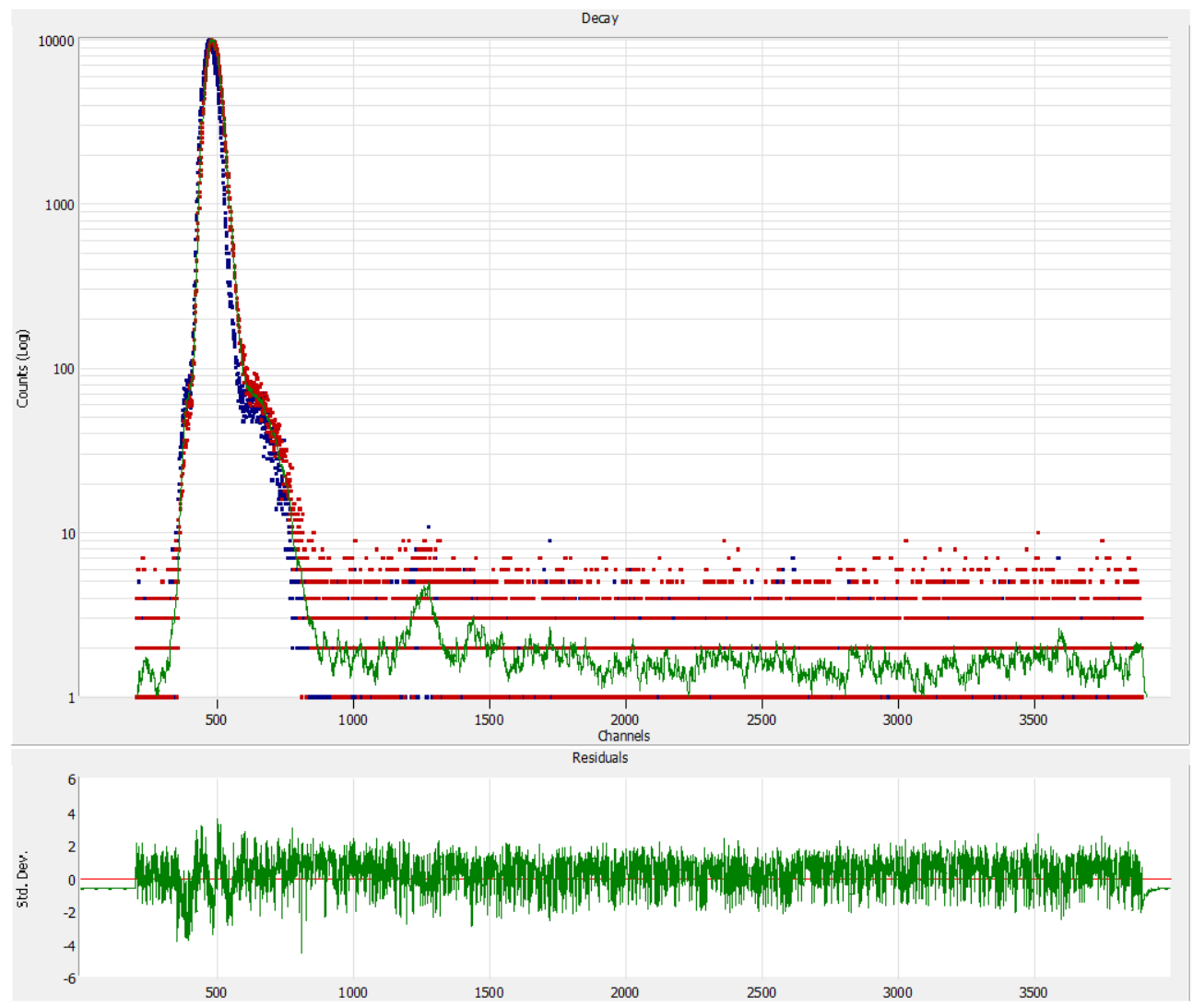

Figure S65. The fluorescence decay fit of TbL4c $\mathbf{c}^{\mathbf{M O M}}\left(\lambda_{\mathrm{em}}=390 \mathrm{~nm}\right.$, PIPES buffer) and residuals of the fit. 


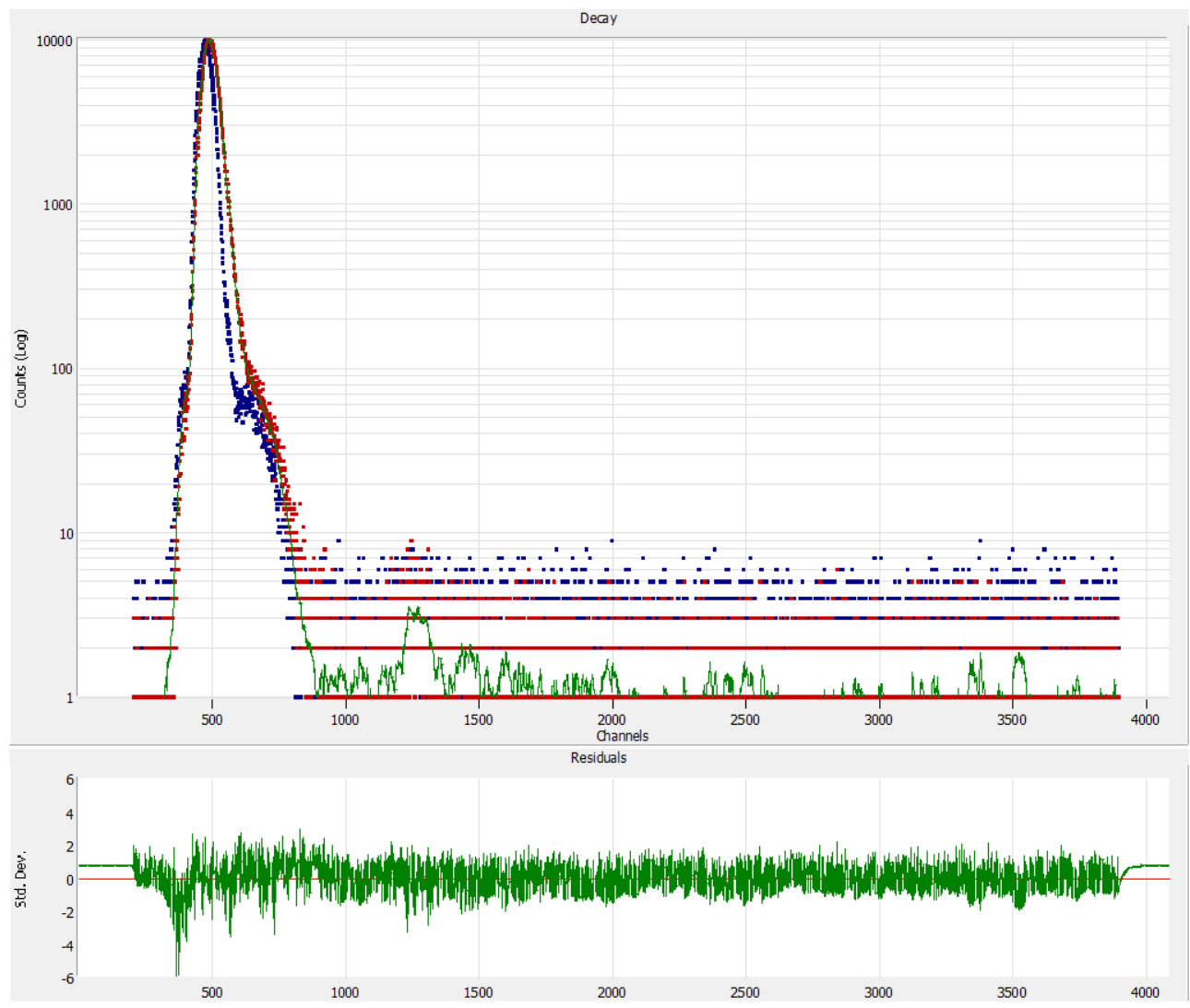

Figure S66. The fluorescence decay fit of $\mathbf{L 1}^{\mathbf{M O M}}\left(\mathrm{pH}=3.70, \lambda_{\mathrm{em}}=390 \mathrm{~nm}\right.$, PIPES buffer) and residuals of the fit.

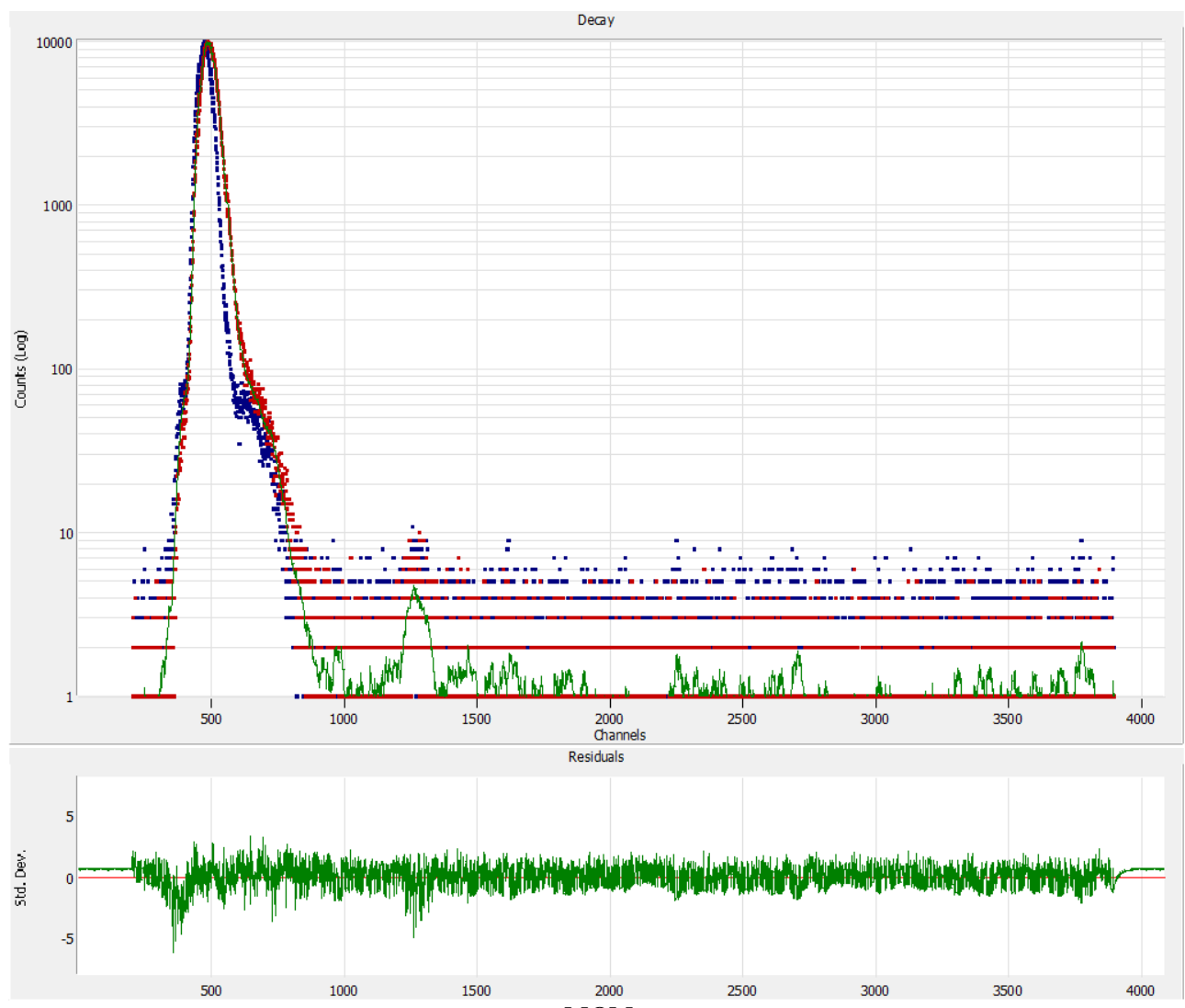

Figure S67. The fluorescence decay fit of $\mathbf{L 1}^{\mathbf{M O M}}\left(\mathrm{pH}=7.75, \lambda_{\mathrm{em}}=390 \mathrm{~nm}\right.$, PIPES buffer $)$ and residuals of the fit. 
Table S21. Residual antenna fluorescence and Eu(III) emission quantum yields from several independent experiments. Measurements were performed in $10 \mathrm{mM}$ aqueous PIPES buffer solutions at $\mathrm{pH} 6.5$ at nominally $10 \mu \mathrm{M}$ concentrations.

\begin{tabular}{|c|c|c|c|c|c|c|}
\hline Complex & $\begin{array}{c}\Phi_{\mathrm{L}}[\%] \\
\mathrm{a}\end{array}$ & $\begin{array}{c}\Phi_{\mathrm{L}, \mathrm{av}}[\%] \\
\mathrm{b}\end{array}$ & $\begin{array}{c}\text { Standard } \\
\text { Deviation }\end{array}$ & $\begin{array}{c}\Phi_{\mathrm{Ln}}[\%] \\
\mathrm{a}\end{array}$ & $\begin{array}{c}\Phi_{\mathrm{Ln}, \mathrm{av}}[\%] \\
\mathrm{b}\end{array}$ & $\begin{array}{l}\text { Standard } \\
\text { Deviation }\end{array}$ \\
\hline \multirow{3}{*}{ EuL1 ${ }^{\text {MOM }}$} & 0.286 & \multirow{3}{*}{0.322} & \multirow{3}{*}{0.038} & 4.91 & \multirow{3}{*}{5.07} & \multirow{3}{*}{0.16} \\
\hline & 0.319 & & & 5.07 & & \\
\hline & 0.362 & & & 5.23 & & \\
\hline \multirow{2}{*}{ EuL2 ${ }^{\text {MOM }}$} & 0.199 & \multirow{2}{*}{0.212} & \multirow{2}{*}{0.018} & 3.64 & \multirow{2}{*}{3.57} & \multirow{2}{*}{0.10} \\
\hline & 0.225 & & & 3.50 & & \\
\hline \multirow{2}{*}{ EuL3 ${ }^{\text {MOM }}$} & 0.103 & \multirow{2}{*}{0.168} & \multirow{2}{*}{0.092} & 2.59 & \multirow{2}{*}{2.55} & \multirow{2}{*}{0.06} \\
\hline & 0.233 & & & 2.50 & & \\
\hline \multirow{2}{*}{ EuL4a ${ }^{\text {MOM }}$} & 0.115 & \multirow{2}{*}{0.114} & \multirow{2}{*}{0.001} & 0.959 & \multirow{2}{*}{0.980} & \multirow{2}{*}{0.029} \\
\hline & 0.113 & & & 1.00 & & \\
\hline \multirow[b]{2}{*}{ EuL4b } & 0.094 & \multirow[b]{2}{*}{0.100} & \multirow[b]{2}{*}{0.008} & 1.63 & \multirow[b]{2}{*}{1.54} & \multirow[b]{2}{*}{0.13} \\
\hline & 0.106 & & & 1.44 & & \\
\hline \multirow{3}{*}{ EuL4c MOM } & 0.201 & \multirow{3}{*}{0.232} & \multirow{3}{*}{0.032} & 2.89 & \multirow{3}{*}{2.71} & \multirow{3}{*}{0.17} \\
\hline & 0.231 & & & 2.70 & & \\
\hline & 0.264 & & & 2.55 & & \\
\hline \multirow{3}{*}{ EuL4a' ${ }^{\text {MOM}}-F$} & 0.310 & \multirow{3}{*}{0.298} & \multirow{3}{*}{0.012} & 7.58 & \multirow{3}{*}{7.47} & \multirow{3}{*}{0.10} \\
\hline & 0.286 & & & 7.43 & & \\
\hline & 0.299 & & & 7.40 & & \\
\hline
\end{tabular}

a Relative to QS $\left(\Phi=0.59^{4}\right)$ in $\mathrm{H}_{2} \mathrm{SO}_{4}(0.05 \mathrm{M}) .{ }^{\mathrm{b}}$ Average quantum yield from two or three independent measurements. 
Table S22. Luminescence lifetimes, decay rates and hydration states of $\mathrm{Eu}$ and $\mathrm{Tb}$ complexes, and the amount of excitation energy lost to $\mathrm{X}-\mathrm{H}$ quenching. Measurements were performed in $10 \mathrm{mM}$ aqueous PIPES buffer solutions at $\mathrm{pH} 6.5$ at nominally $10 \mu \mathrm{M}$ concentrations.

\begin{tabular}{|c|c|c|c|c|c|c|}
\hline Complex & $\tau_{\mathrm{H} 2 \mathrm{O}}{ }^{[\mathrm{a}]}$ & $k_{\mathrm{H} 2 \mathrm{O}^{[\mathrm{b}]}}$ & $\tau_{\mathrm{D} 2 \mathrm{O}}^{[\mathrm{a}]}$ & $k_{\mathrm{D} 2 \mathrm{O}}{ }^{[\mathrm{b}]}$ & loss $[\mathrm{c}]$ & $q^{[\mathrm{d}]}$ \\
\hline EuL1 $^{\text {Me }}$ & 0.596 & 1.68 & 1.95 & 0.51 & 70 & 1.0 \\
\hline $\mathbf{E u L 4 a}^{\mathrm{Me}}$ & 0.451 & 2.22 & 1.59 & 0.62 & 72 & 0.7 \\
\hline EuL1CF3 & 0.604 & 1.66 & 2.01 & 0.50 & 70 & 1.0 \\
\hline EuL4a $a^{C F 3}$ & 0.433 & 2.31 & 1.77 & 0.57 & 75 & 1.2 \\
\hline TbL1 ${ }^{\text {MOM }}$ & 1.07 & 0.935 & 1.49 & 0.67 & 28 & 1.0 \\
\hline TbL2 ${ }^{\text {MOM }}$ & 1.04 & 0.962 & 1.50 & 0.67 & 31 & 1.2 \\
\hline TbL3 $3^{\text {MOM }}$ & 0.972 & 1.03 & 1.43 & 0.70 & 32 & 1.4 \\
\hline TbL4a $\mathbf{a}^{\text {MOM }}$ & 0.956 & 1.05 & 1.33 & 0.75 & 28 & 1.2 \\
\hline TbL4b ${ }^{\text {MOM }}$ & 0.983 & 1.02 & 1.41 & 0.71 & 30 & 1.2 \\
\hline TbL4c & 1.01 & 0.990 & 1.32 & 0.76 & 23 & 0.9 \\
\hline EuL1 $^{\text {MOM }}$ & 0.605 & 1.65 & 2.16 & 0.46 & 72 & 1.0 \\
\hline EuL2 ${ }^{\text {MOM }}$ & 0.572 & 1.75 & 2.14 & 0.47 & 73 & 1.0 \\
\hline EuL3 $3^{\text {MOM }}$ & 0.522 & 1.92 & 1.95 & 0.51 & 73 & 0.9 \\
\hline EuL4aMOM & 0.439 & 2.28 & 1.44 & 0.69 & 70 & 1.0 \\
\hline EuL4b' & 0.498 & 2.01 & 1.68 & 0.60 & 70 & 1.0 \\
\hline EuL4c ${ }^{\text {MOM }}$ & 0.549 & 1.82 & 1.65 & 0.61 & 66 & 1.1 \\
\hline EuL4c $^{M O M}$-F & 0.98 & 1.02 & 2.37 & 0.42 & 59 & 0 \\
\hline
\end{tabular}

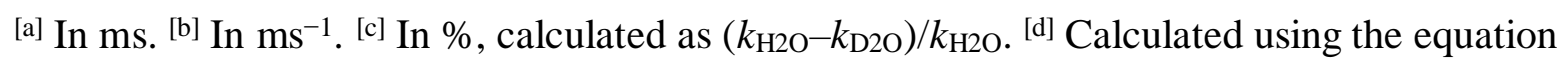
$q=5\left(1 / \tau_{\mathrm{H} 2 \mathrm{O}}-1 / \tau_{\mathrm{D} 2 \mathrm{O}}-0.06\right)$ for $\mathrm{Tb}$, and $q=1.2\left(1 / \tau_{\mathrm{H} 2 \mathrm{O}}-1 / \tau_{\mathrm{D} 2 \mathrm{O}}-0.25-n \times 0.075\right)$, where $n$ is the number of nearby N-H oscillators, for Eu. 
Table S23. The loss of excitation energy due to X-H oscillators from excited Eu(III) in selected $q=1$ and $q=0$ complexes from the literature.

\begin{tabular}{|c|c|c|c|c|c|c|c|}
\hline Complex & $\tau_{\mathrm{H} 2 \mathrm{O}^{[\mathrm{a}]}}$ & $k_{\mathrm{H} 2 \mathrm{O}^{[\mathrm{b}]}}$ & $\tau_{\mathrm{D} 2 \mathrm{O}^{[\mathrm{a}]}}$ & $k_{\mathrm{D} 2 \mathrm{O}^{[\mathrm{b}]}}$ & loss $[\mathrm{c}]$ & $q^{[\mathrm{d}]}$ & Reference \\
\hline EuL $^{S 1}$ & 0.625 & 1.6 & 1.85 & 0.54 & 66 & 1 & 10 \\
\hline $\operatorname{EuL}^{\mathrm{S2}}$ & 0.625 & 1.6 & 1.85 & 0.54 & 66 & 1 & 10 \\
\hline EuL $^{S 3}$ & 0.550 & 1.82 & 2.13 & 0.47 & 74 & 1 & 10 \\
\hline EuL $^{S 4}$ & 0.518 & 1.93 & 2.17 & 0.46 & 76 & 1 & 10 \\
\hline EuL $^{S 5}$ & 0.581 & 1.72 & 2.38 & 0.42 & 76 & 1 & 10 \\
\hline $\mathrm{EuL}^{\mathrm{S6}}$ & 0.65 & 1.54 & 2.18 & 0.46 & 70 & 1.0 & 11 \\
\hline $\mathbf{E u L}^{\mathrm{S7}}$ & 0.66 & 1.52 & 2.16 & 0.46 & 69 & 1.0 & 11 \\
\hline EuL $^{S 8}$ & 0.65 & 1.54 & 2.17 & 0.46 & 70 & 1.0 & 11 \\
\hline EuL $^{S 9}$ & 0.66 & 1.52 & 2.16 & 0.46 & 69 & 1.0 & 11 \\
\hline EuL $L^{S 10}$ & 0.46 & 2.17 & 1.64 & 0.61 & 72 & 1.5 & 12 \\
\hline EuL ${ }^{S 11}$ & 0.61 & 1.64 & 2.35 & 0.43 & 74 & 1.2 & 12 \\
\hline EuL ${ }^{S 12}$ & 0.63 & 1.59 & 2.38 & 0.42 & 74 & 1 & 12 \\
\hline EuL ${ }^{S 13}$ & 1.25 & 0.80 & 1.85 & 0.54 & 32 & 0 & 10 \\
\hline EuL ${ }^{S 14}$ & 1.67 & 0.60 & 2.08 & 0.48 & 20 & 0 & 10 \\
\hline EuL $L^{\mathrm{S15}}$ & 1.00 & 1.00 & 1.38 & 0.73 & 27 & 0 & 13 \\
\hline
\end{tabular}

${ }^{[\mathrm{a}]}$ In ms. ${ }^{[\mathrm{b}]} \mathrm{In} \mathrm{ms}^{-1} .{ }^{[\mathrm{c}]} \mathrm{In} \%$, calculated as $\left(k_{\mathrm{H} 2 \mathrm{O}}-k_{\mathrm{D} 2 \mathrm{O}}\right) / k_{\mathrm{H} 2 \mathrm{O}}{ }^{[\mathrm{d}]}$ Value taken from the reference.

Table S24. The asymmetry (r) values of the complexes.

\begin{tabular}{llll}
\hline Complex & $I_{J=2}{ }^{[\mathrm{a}]}$ & $I_{J=1}[\mathrm{~b}]$ & $r^{[\mathrm{c}]}$ \\
\hline EuL1 MOM & $2.72283 \times 10^{6}$ & $2.24233 \times 10^{6}$ & 1.21 \\
EuL2 $^{\text {MOM }}$ & $1.90521 \times 10^{6}$ & $1.55417 \times 10^{6}$ & 1.23 \\
EuL3 $^{\text {MOM }}$ & $1.62362 \times 10^{6}$ & $1.06311 \times 10^{6}$ & 1.53 \\
EuL4a $^{\text {MOM }}$ & 645706 & 422657 & 1.53 \\
EuL4b $^{\text {MOM }}$ & 824218 & 636398 & 1.30 \\
EuL4c $^{\text {MOM }}$ & $1.51759 \times 10^{6}$ & $1.07783 \times 10^{6}$ & 1.41 \\
\hline EuL4a $^{\text {MOM}}-\mathbf{F}$ & $6.89858 \times 10^{6}$ & $3.07189 \times 10^{6}$ & 2.25 \\
\hline
\end{tabular}

[a] Integral of the emission band corresponding to ${ }^{5} \mathrm{D}_{0} \rightarrow{ }^{7} \mathrm{~F}_{2}$ transition (from 604 to $640 \mathrm{~nm}$ ).

${ }^{[b]}$ Integral of the emission band corresponding to ${ }^{5} \mathrm{D}_{0} \rightarrow{ }^{7} \mathrm{~F}_{1}$ transition (from 582 to $603 \mathrm{~nm}$ ).

[c] The ratio of $I_{J=2} / I_{J=1}$. 

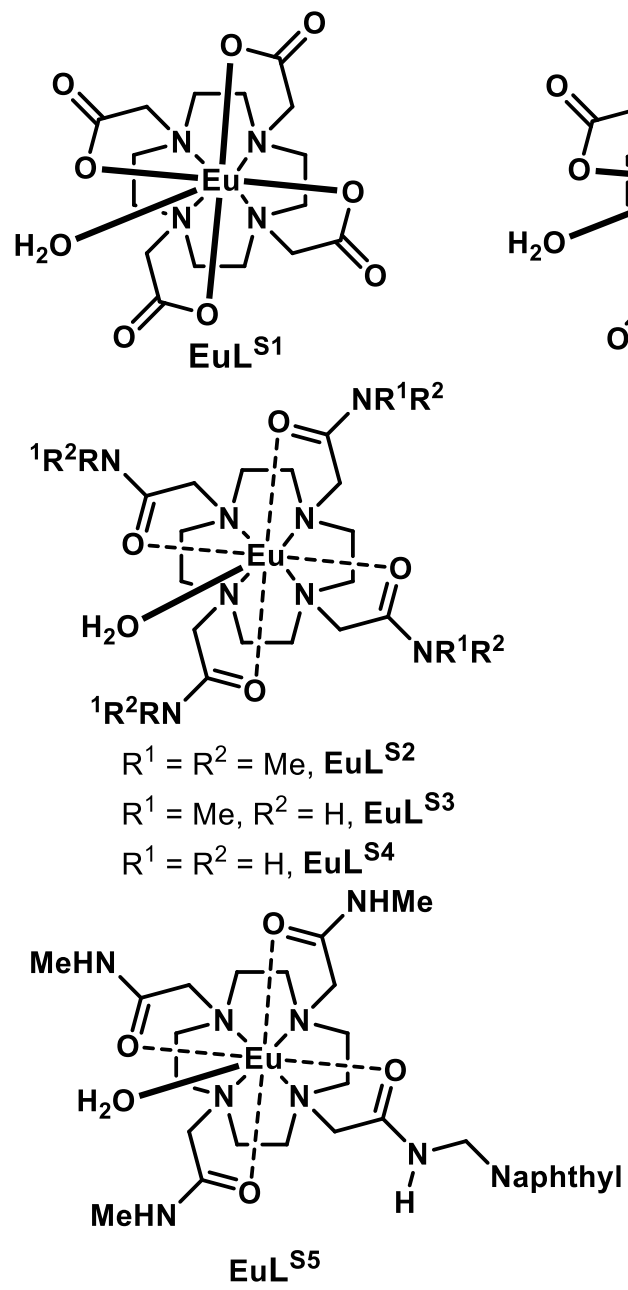

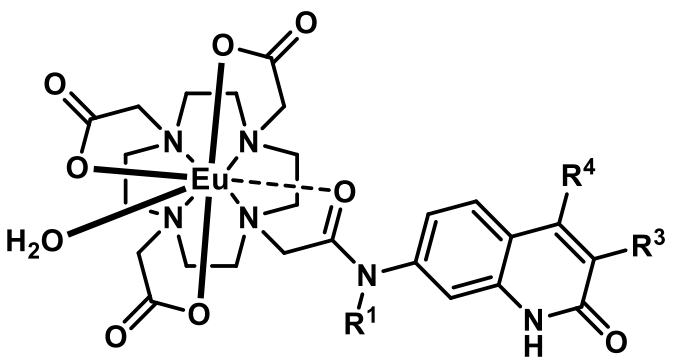

$\mathrm{R}^{1}=\mathrm{CH}_{2} \mathrm{CO}_{2}^{-}, \mathrm{R}^{3}=\mathrm{H}, \mathrm{R}^{4}=\mathrm{Me}, \mathrm{EuL}^{\mathrm{S}}$

$\mathrm{R}^{1}=\mathrm{CH}_{2} \mathrm{CO}_{2}^{-}, \mathrm{R}^{3}=\mathrm{H}, \mathrm{R}^{4}=\mathrm{CF}_{3}$, EuL ${ }^{\mathrm{S7}}$

$\mathrm{R}^{1}=\mathrm{CH}_{2} \mathrm{CO}_{2}^{-}, \mathrm{R}^{3}=\mathrm{H}, \mathrm{R}^{4}=$ MOM, EuL ${ }^{\mathrm{s8}}$

$\mathrm{R}^{1}=\mathrm{CH}_{2} \mathrm{CO}_{2}^{-}, \mathrm{R}^{3}=\mathrm{CH}_{2} \mathrm{CO}_{2} \mathrm{Et}, \mathrm{R}^{4}=\mathrm{Me}$, EuL ${ }^{\mathrm{Sg}}$

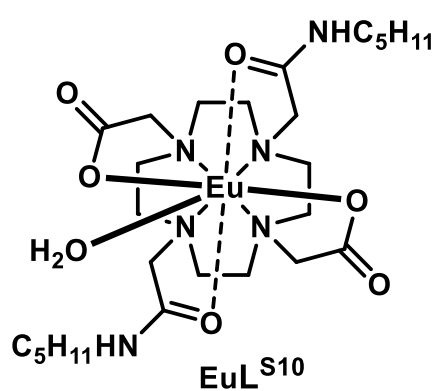

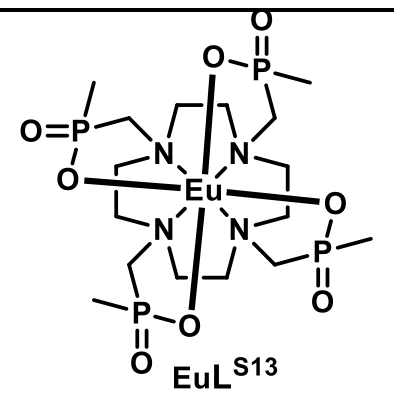
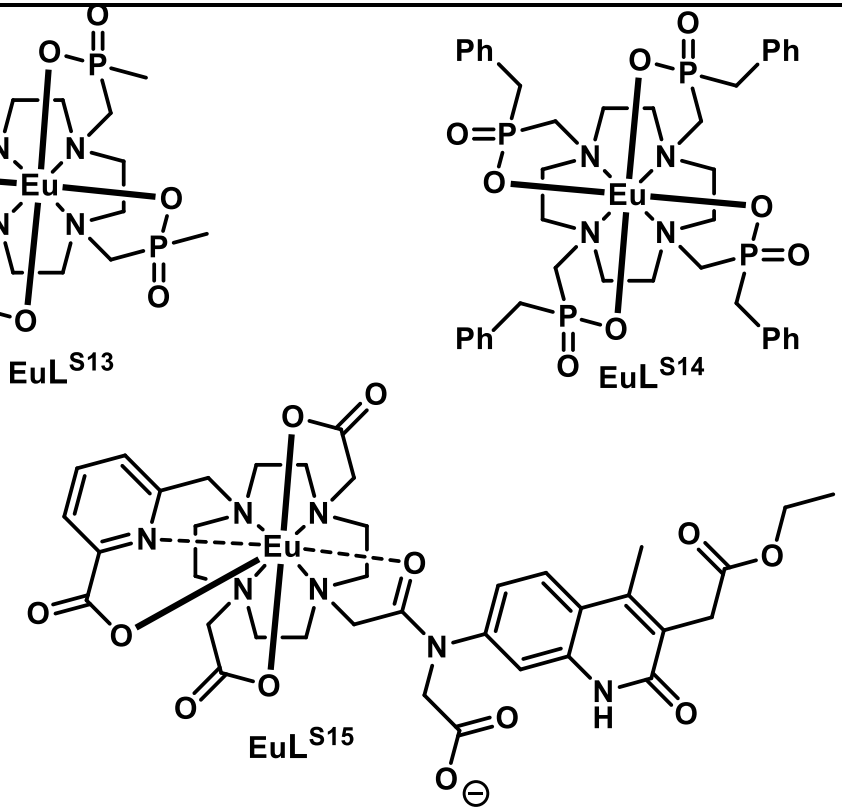

Chart S1. The structures of the complexes listed in Table S23. 
Table S25. Residual antenna fluorescence and Eu(III) emission quantum yields from several independent experiments in $\mathrm{D}_{2} \mathrm{O}$. Measurements were performed in $10 \mathrm{mM}$ PIPES buffer in $\mathrm{D}_{2} \mathrm{O}$ solutions at $\mathrm{pH} 6.5$ at nominally $10 \mu \mathrm{M}$ concentrations.

\begin{tabular}{|c|c|c|c|c|c|c|}
\hline Complex & $\Phi_{\mathrm{L}}[\%]$ & $\begin{array}{c}\Phi_{\mathrm{L}, \mathrm{av}}[\%] \\
{[\mathrm{b}]}\end{array}$ & $\begin{array}{l}\text { Standard } \\
\text { Deviation }\end{array}$ & $\Phi_{\mathrm{Ln}}[\%]$ & $\Phi_{\mathrm{Ln}, \mathrm{av}}[\%]$ & $\begin{array}{l}\text { Standard } \\
\text { Deviation }\end{array}$ \\
\hline \multirow{3}{*}{ EuL1 ${ }^{\text {MOM }}$} & 0.355 & \multirow{3}{*}{0.353} & \multirow{3}{*}{0.002} & 17.6 & \multirow{3}{*}{18.0} & \multirow{3}{*}{0.4} \\
\hline & 0.351 & & & 18.1 & & \\
\hline & 0.352 & & & 18.4 & & \\
\hline \multirow{2}{*}{ EuL2 ${ }^{\text {MOM }}$} & 0.286 & \multirow{2}{*}{0.290} & \multirow{2}{*}{0.005} & 13.6 & \multirow{2}{*}{13.6} & \multirow{2}{*}{0.1} \\
\hline & 0.293 & & & 13.5 & & \\
\hline \multirow{2}{*}{ EuL3 ${ }^{\text {MOM }}$} & 0.258 & \multirow{2}{*}{0.262} & \multirow{2}{*}{0.006} & 10.4 & \multirow{2}{*}{10.4} & \multirow{2}{*}{0} \\
\hline & 0.266 & & & 10.4 & & \\
\hline \multirow{2}{*}{ EuL4a } & 0.105 & \multirow{2}{*}{0.106} & \multirow{2}{*}{0.001} & 4.22 & \multirow{2}{*}{3.98} & \multirow{2}{*}{0.35} \\
\hline & 0.107 & & & 3.73 & & \\
\hline \multirow{3}{*}{ EuL4b } & 0.107 & \multirow{3}{*}{0.114} & \multirow{3}{*}{0.013} & 5.83 & \multirow{3}{*}{5.77} & \multirow{3}{*}{0.11} \\
\hline & 0.129 & & & 5.83 & & \\
\hline & 0.107 & & & 5.64 & & \\
\hline \multirow{3}{*}{ EuL4c } & 0.256 & \multirow{3}{*}{0.273} & \multirow{3}{*}{0.015} & 8.19 & \multirow{3}{*}{8.18} & \multirow{3}{*}{0.01} \\
\hline & 0.283 & & & 8.17 & & \\
\hline & 0.279 & & & 8.18 & & \\
\hline
\end{tabular}

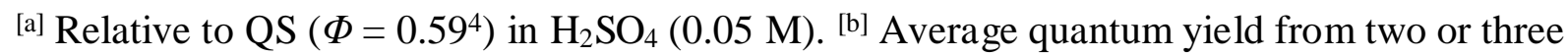
independent measurements.

Table S26. Luminescence lifetimes and decay rates of EuL ${ }^{\text {MOM }}$ complexes in $\mathrm{D}_{2} \mathrm{O}$. Measurements were performed in 10 mM PIPES buffer in $\mathrm{D}_{2} \mathrm{O}$ solutions at $\mathrm{pH} 6.5$ at nominally $10 \mu \mathrm{M}$ concentrations.

\begin{tabular}{cccccc}
\hline Complex & $\tau_{\text {rad,Ln }}{ }^{[\mathrm{a}]}$ & $\tau_{\mathrm{obs}}$ & $k_{\mathrm{D} 2 \mathrm{O}}{ }^{[\mathrm{b}]}$ & $k_{\mathrm{rad}, \mathrm{Ln}}{ }^{[\mathrm{b}]}$ & $k_{\mathrm{nr}, \mathrm{Ln}}{ }^{[\mathrm{b}][\mathrm{c}]}$ \\
\hline EuL1 $^{\text {MOM }}$ & 5.22 & 2.16 & 0.46 & 0.192 & 0.27 \\
EuL2 $^{\text {MOM }}$ & 5.20 & 2.14 & 0.47 & 0.192 & 0.28 \\
EuL3 $^{\text {MOM }}$ & 4.99 & 1.95 & 0.51 & 0.201 & 0.31 \\
EuL4a $^{\text {MOM }}$ & 5.00 & 1.44 & 0.69 & 0.200 & 0.49 \\
EuL4b $^{\text {MOM }}$ & 5.20 & 1.68 & 0.60 & 0.192 & 0.41 \\
EuL4c $^{\text {MOM }}$ & 4.96 & 1.65 & 0.61 & 0.201 & 0.41 \\
EuL4a $^{\text {MOM_F }}{ }^{[\mathrm{d}]}$ & 4.87 & 2.37 & 0.42 & 0.205 & 0.22
\end{tabular}

${ }^{\text {[a] }}$ In ms, determined using Eq. 1 and 2 (see manuscript). ${ }^{[\mathrm{b}]} \mathrm{In} \mathrm{ms}^{-1} \cdot{ }^{[\mathrm{c}]} k_{\mathrm{nr}}=1 / \tau_{\mathrm{obs}}-k_{\text {rad. }}{ }^{[\mathrm{d}]}$ Formed by the addition of excess KF ( $0.1 \mathrm{M}, 10^{4}$-fold excess) to a solution of EuL4a $\mathbf{a}^{\text {MOM }}$. 
Table S27. Photophysical properties of EuL4aMOM.F in $\mathrm{H}_{2} \mathrm{O}$. [a]

\begin{tabular}{llccc}
\hline$\Phi_{\mathrm{L}}{ }^{[\mathrm{b}]}$ & $\Phi_{\mathrm{Ln}}{ }^{[\mathrm{b}]}$ & $\boldsymbol{\tau}_{\boldsymbol{r a d}, \boldsymbol{L n}}{ }^{[\mathrm{d}]}$ & $\boldsymbol{\Phi}_{\boldsymbol{L n}}^{\boldsymbol{L n}}{ }^{[\mathrm{e}]}$ & $\boldsymbol{\eta}_{\boldsymbol{s e n \boldsymbol { s }}}{ }^{[\mathrm{e}]}$ \\
\hline $0.298 \pm 0.012\left(2.61^{[\mathrm{c}]}\right)$ & $7.47 \pm 0.10(7.62)^{[\mathrm{c}]}$ & $4.84(0.98)$ & $20.3(2.29)$ & $36.8(3.35)$ \\
\hline
\end{tabular}

${ }^{\text {[a] }}$ Formed by the addition of excess $\mathrm{KF}\left(0.1 \mathrm{M}, 10^{4}\right.$-fold excess) to a solution of EuL4a $\mathbf{a}^{\text {MOM }}$. In $10 \mathrm{mM}$ aqueous PIPES buffer solutions at pH 6.5 at nominally $10 \mu \mathrm{M}$ concentrations. ${ }^{[\mathrm{b}]} \mathrm{In}$ $\%$, relative to quinine sulfate $(\Phi=0.59)$ in $\mathrm{H}_{2} \mathrm{SO}_{4}(0.05 \mathrm{M}) .{ }^{4}{ }^{[\mathrm{c}]}$ Mean \pm standard deviation for three independent measurements. In parentheses: fold increase compared to EuL4a $\mathbf{a}^{\mathbf{M O M}}$ without added KF. ${ }^{[d]}$ Calculated using Eq. 1 and 2, ${ }^{14}$ in parentheses change relative to the solution without added KF. In ms. ${ }^{[e]}$ In \%, determined using Eq. 1 and 2. In parentheses: fold increase compared to EuL4a $\mathbf{a M}^{\text {MOM }}$ without added KF.

Table S28. Photophysical properties of EuL4a ${ }^{\mathrm{MOM}}-\mathbf{F}$ in $\mathrm{D}_{2} \mathrm{O}$. [a]

\begin{tabular}{rrrr}
\hline$\Phi_{\mathrm{L}}{ }^{[\mathrm{b}]}$ & $\Phi_{\mathrm{Ln}}{ }^{[\mathrm{b}]}$ & $\boldsymbol{\Phi}_{\boldsymbol{L n}}^{\boldsymbol{L n}[\mathrm{d}]}$ & $\boldsymbol{\eta}_{\boldsymbol{s e n s}}{ }^{[\mathrm{d}]}$ \\
\hline 0.287 & $21.2(2.8)^{[\mathrm{c}]}$ & $48.7(2.40)$ & $43.5(1.18)$ \\
\hline
\end{tabular}

${ }^{\text {[a] }}$ Formed by the addition of excess $\mathrm{KF}\left(0.1 \mathrm{M}, 10^{4}\right.$-fold excess) to a solution of EuL4a $\mathbf{a}^{\text {MOM }}$. In $10 \mathrm{mM}$ PIPES buffer solutions in $\mathrm{D}_{2} \mathrm{O}$ at $\mathrm{pH} 6.5$ at nominally $10 \mu \mathrm{M}$ concentrations. ${ }^{[\mathrm{b}]} \mathrm{In}$ $\%$, relative to quinine sulfate $(\Phi=0.59)$ in $\mathrm{H}_{2} \mathrm{SO}_{4}(0.05 \mathrm{M}) .{ }^{4}{ }^{[c]}$ Fold increase compared to the solution in $\mathrm{H}_{2} \mathrm{O} .{ }^{[d]}$ Calculated using Eq. 1 and $2,{ }^{14}$ in parentheses change relative to the solution in $\mathrm{H}_{2} \mathrm{O}$. 


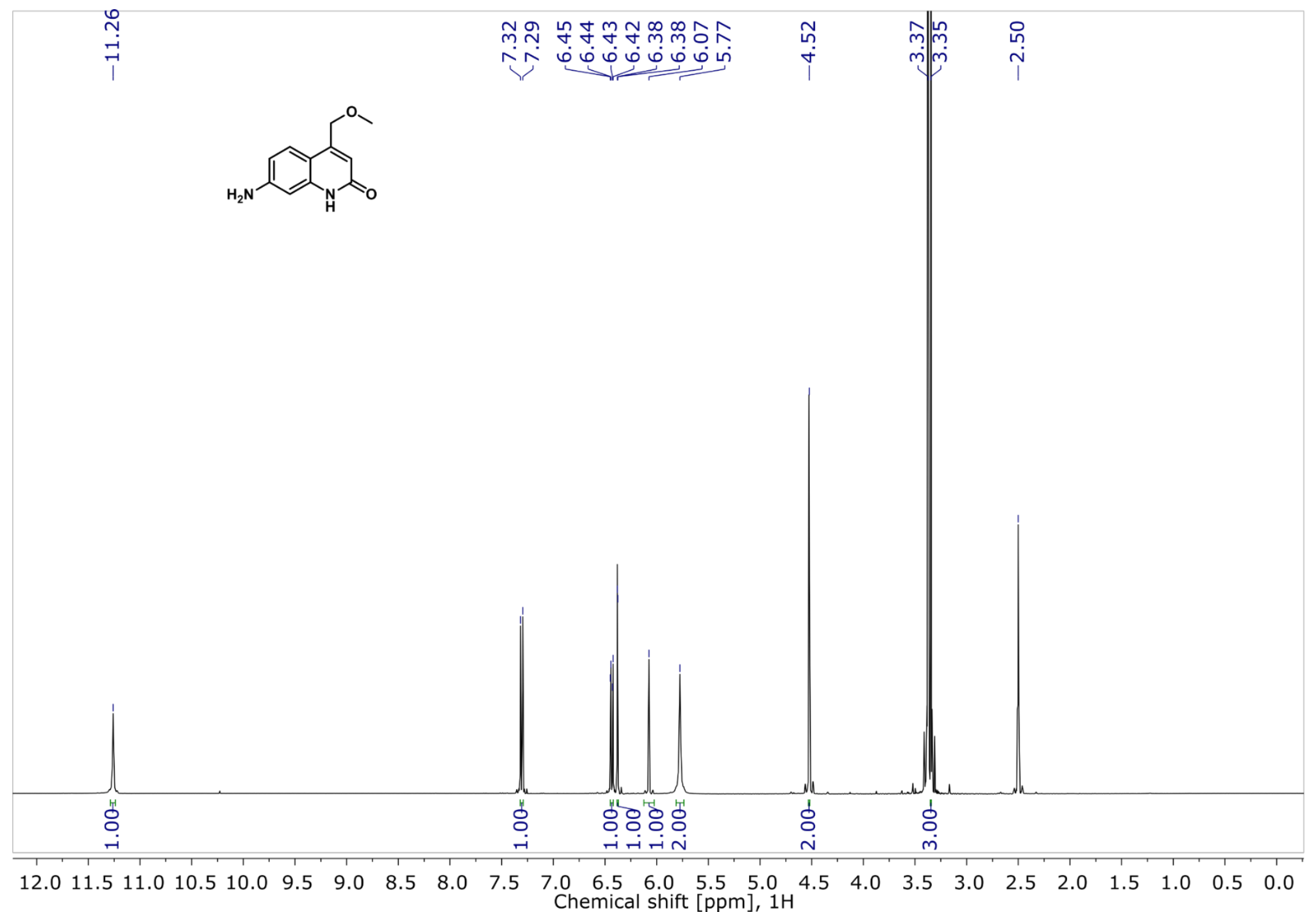

Figure S68. ${ }^{1} \mathrm{H}$ NMR spectrum of 7-amino-4-(methoxymethyl)quinolin-2(1H)-one (400 MHz, DMSO- $\left.d_{6}\right)$. 


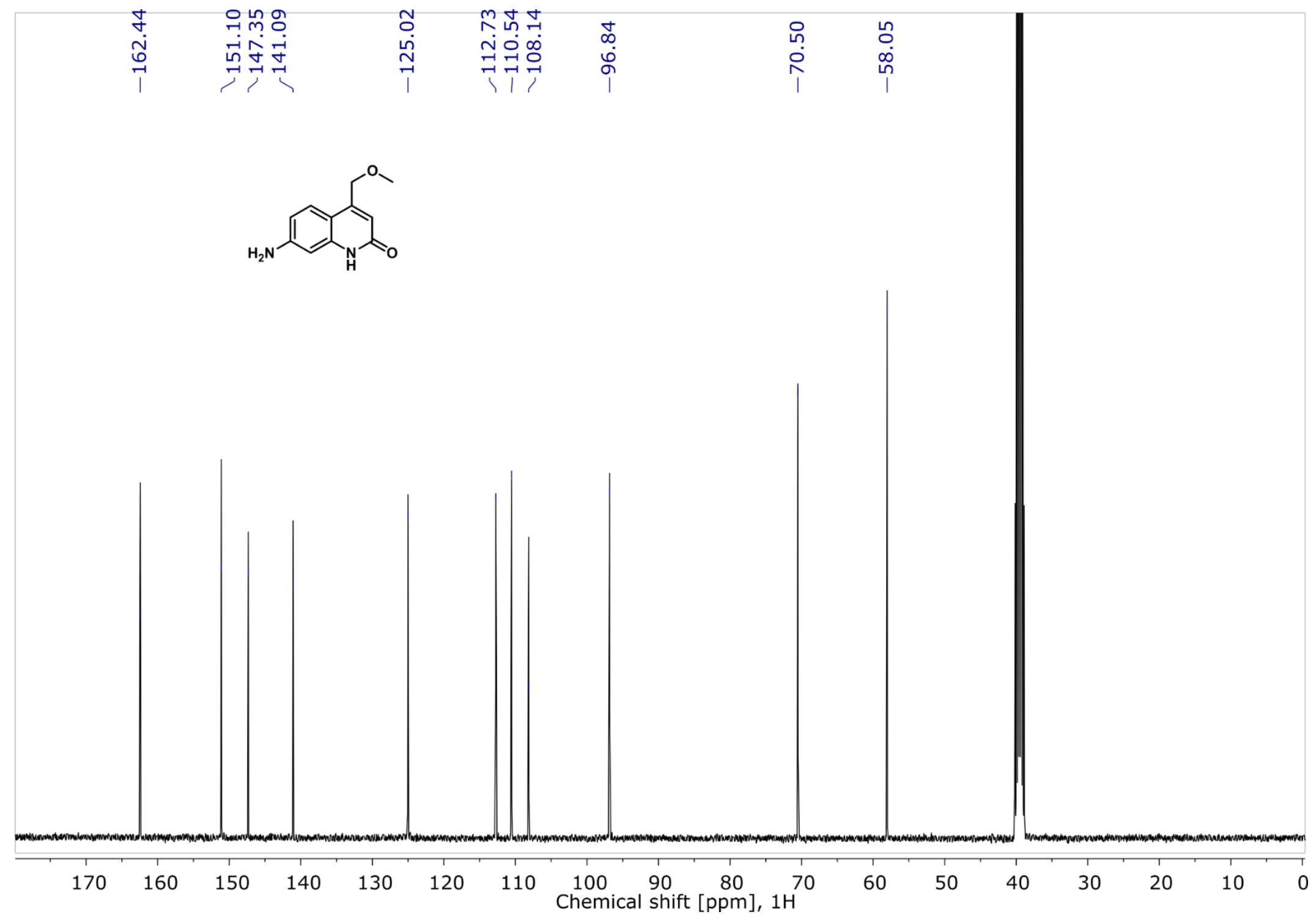

Figure S69. ${ }^{13} \mathrm{C}$ NMR spectrum of 7-amino-4-(methoxymethyl)quinolin-2(1H)-one (101 MHz, DMSO-d6). 


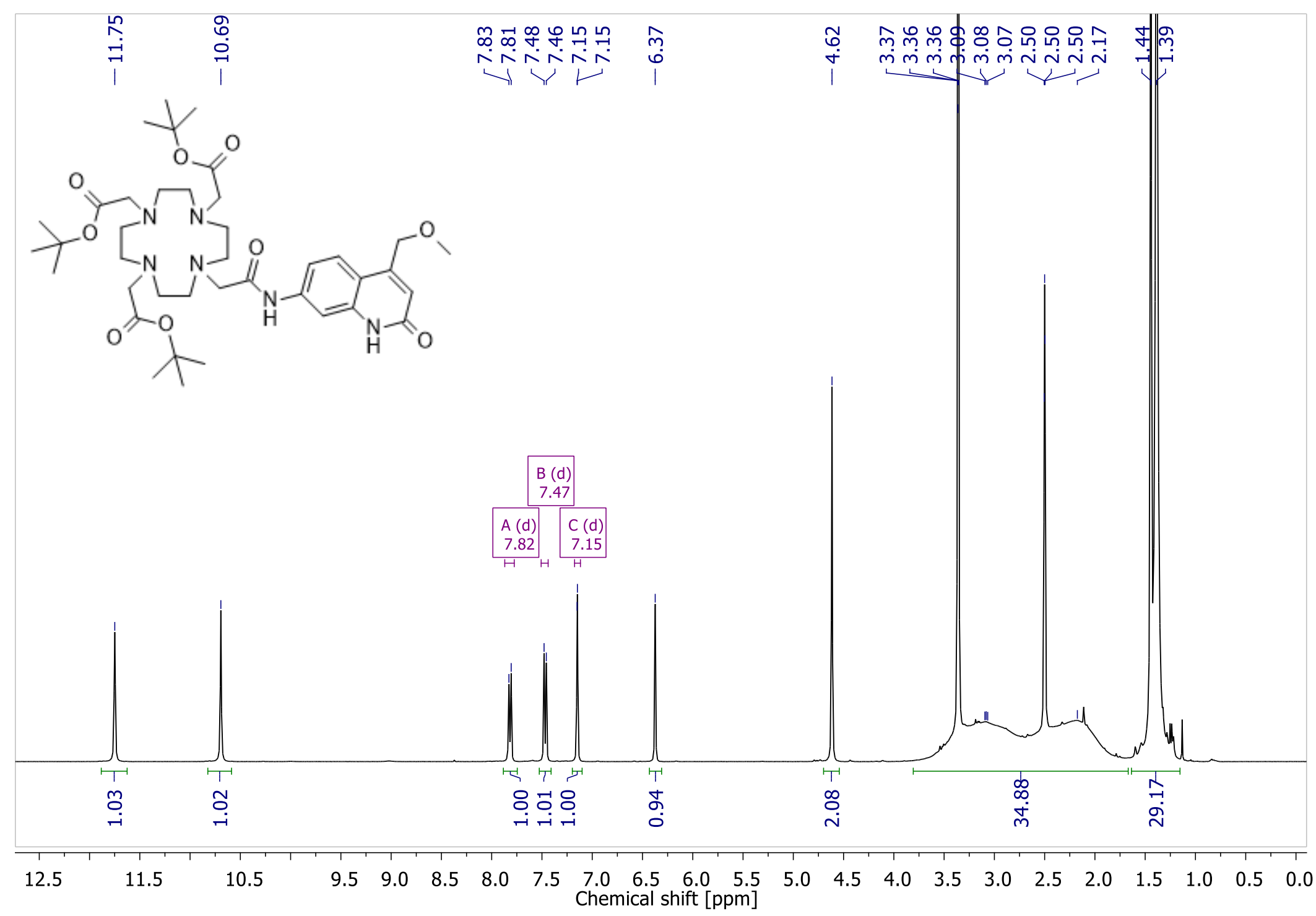

Figure S70. ${ }^{1} \mathrm{H}$ NMR spectrum of Protected L1 ${ }^{\text {MOM }}$ (400 MHz, DMSO- $d_{6}$ ). 


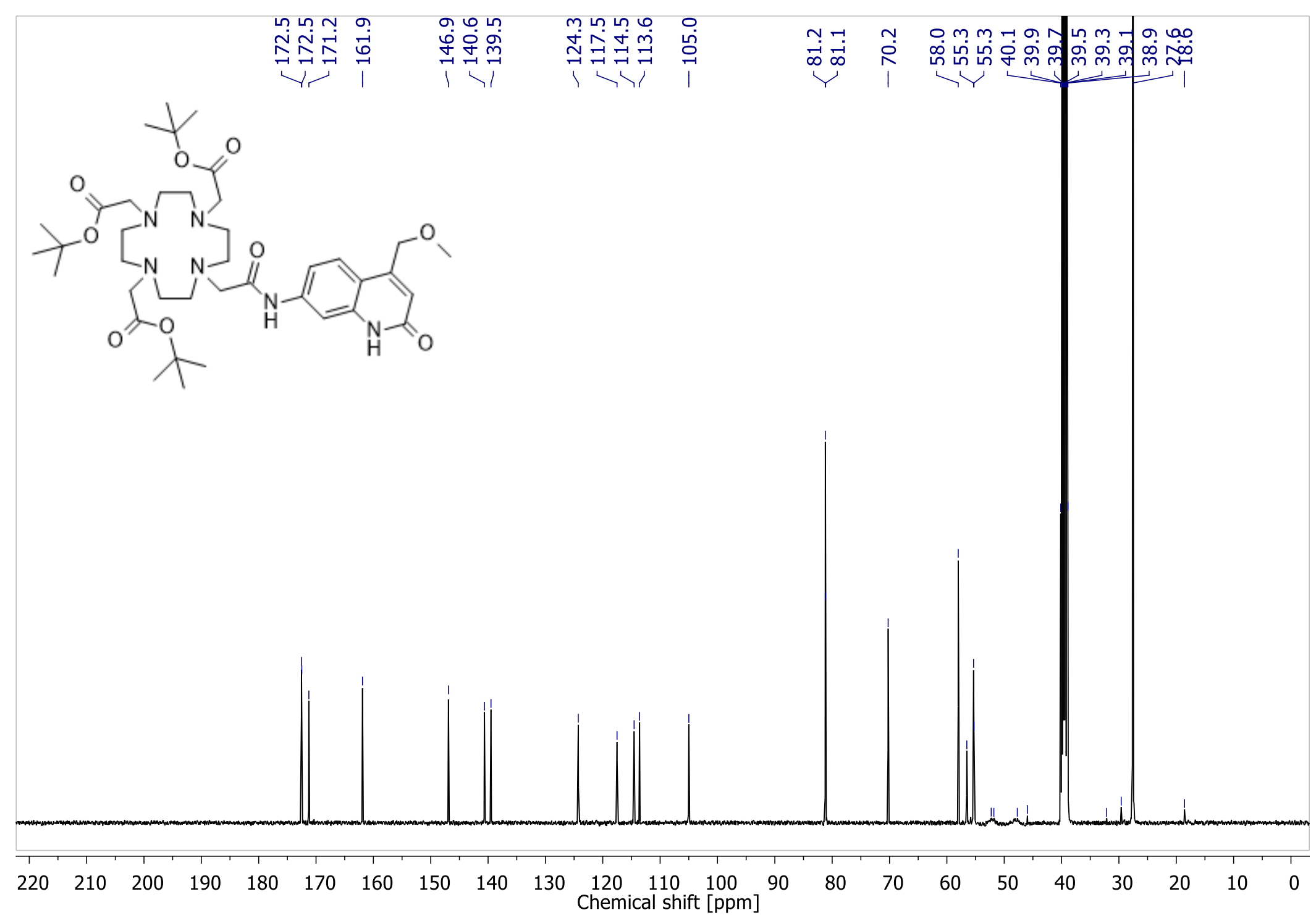

Figure S71. ${ }^{13} \mathrm{C}$ NMR spectrum of Protected L1 ${ }^{\text {MOM }}$ (101 MHz, DMSO- $d_{6}$ ). 


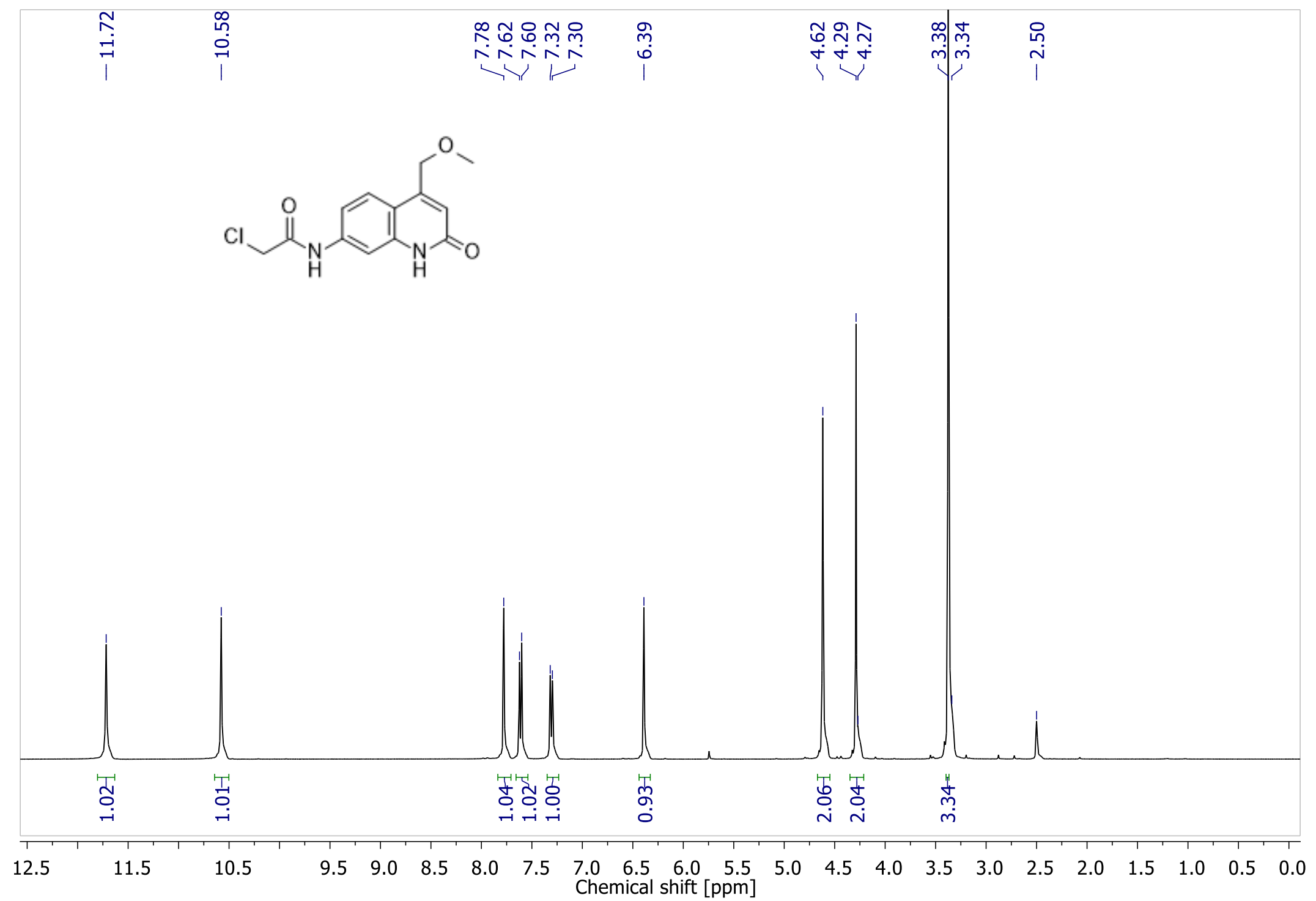

Figure S72. ${ }^{1} \mathrm{H}$ NMR spectrum of 2 (400 MHz, DMSO- $\left.d_{6}\right)$. 


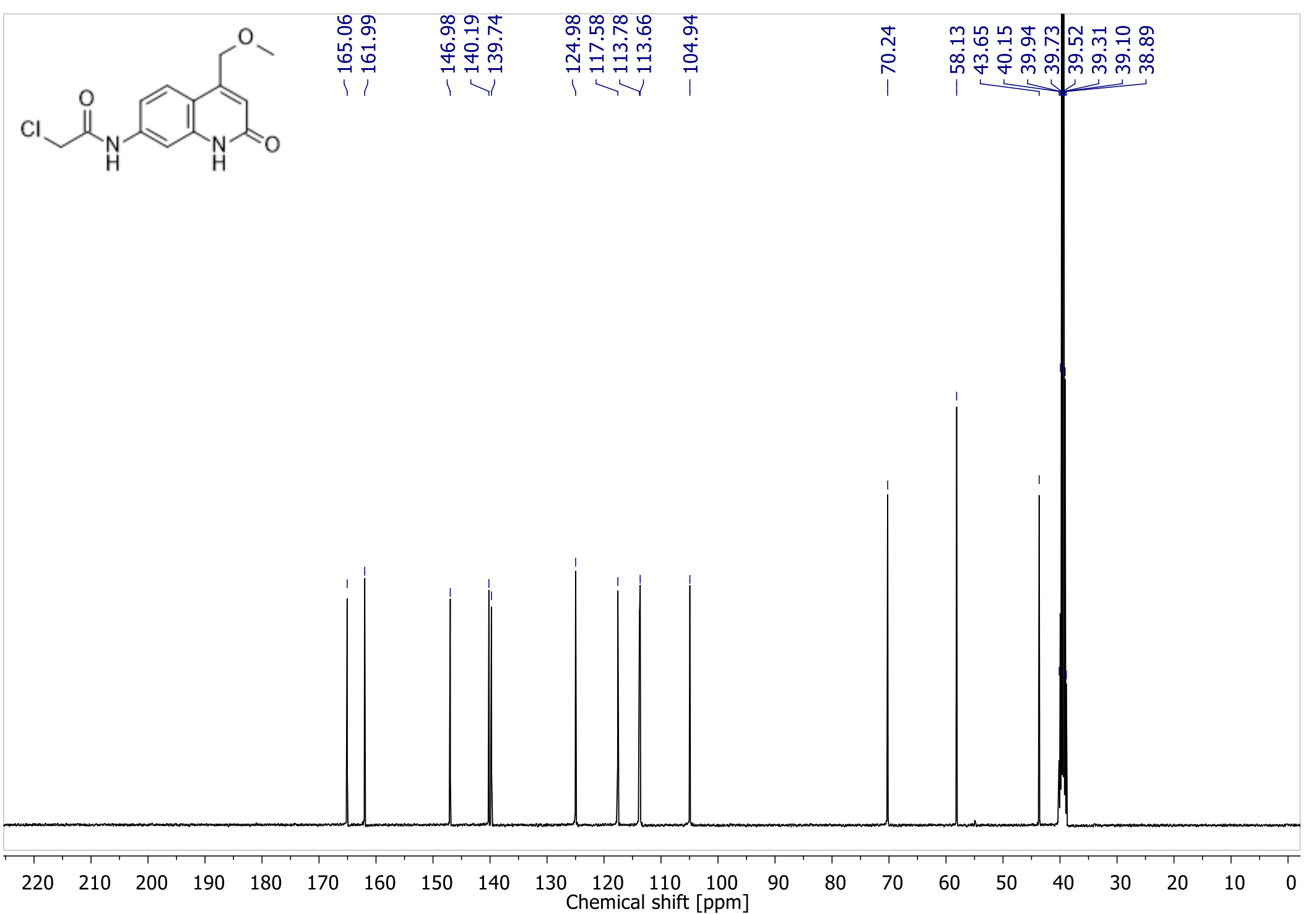

Figure S73. ${ }^{13} \mathrm{C}$ NMR spectrum of 2 (101 MHz, DMSO- $\left.d_{6}\right)$. 


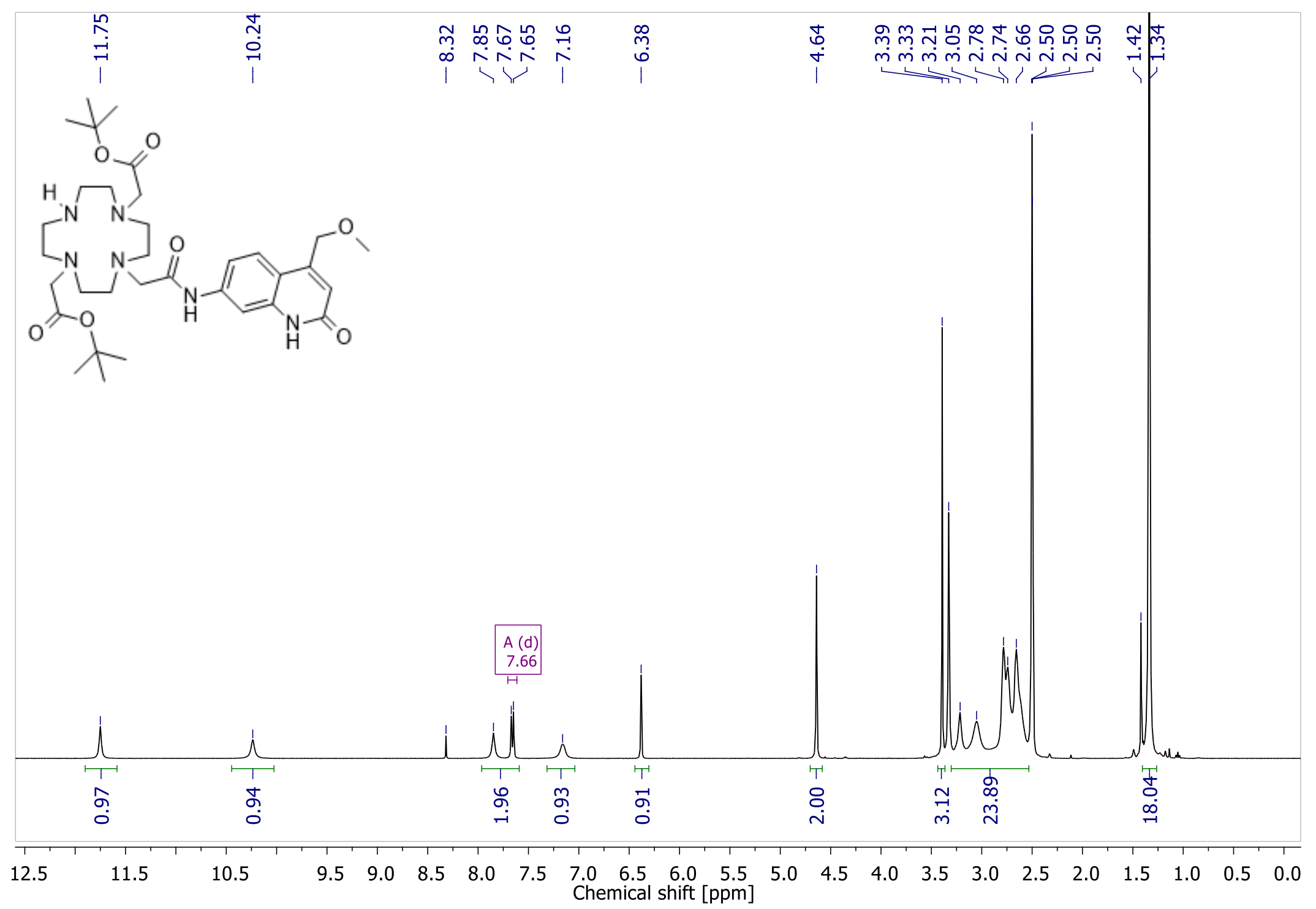

Figure S74. ${ }^{1} \mathrm{H}$ NMR spectrum of 3 (400 MHz, DMSO- $d_{6}$ ). 


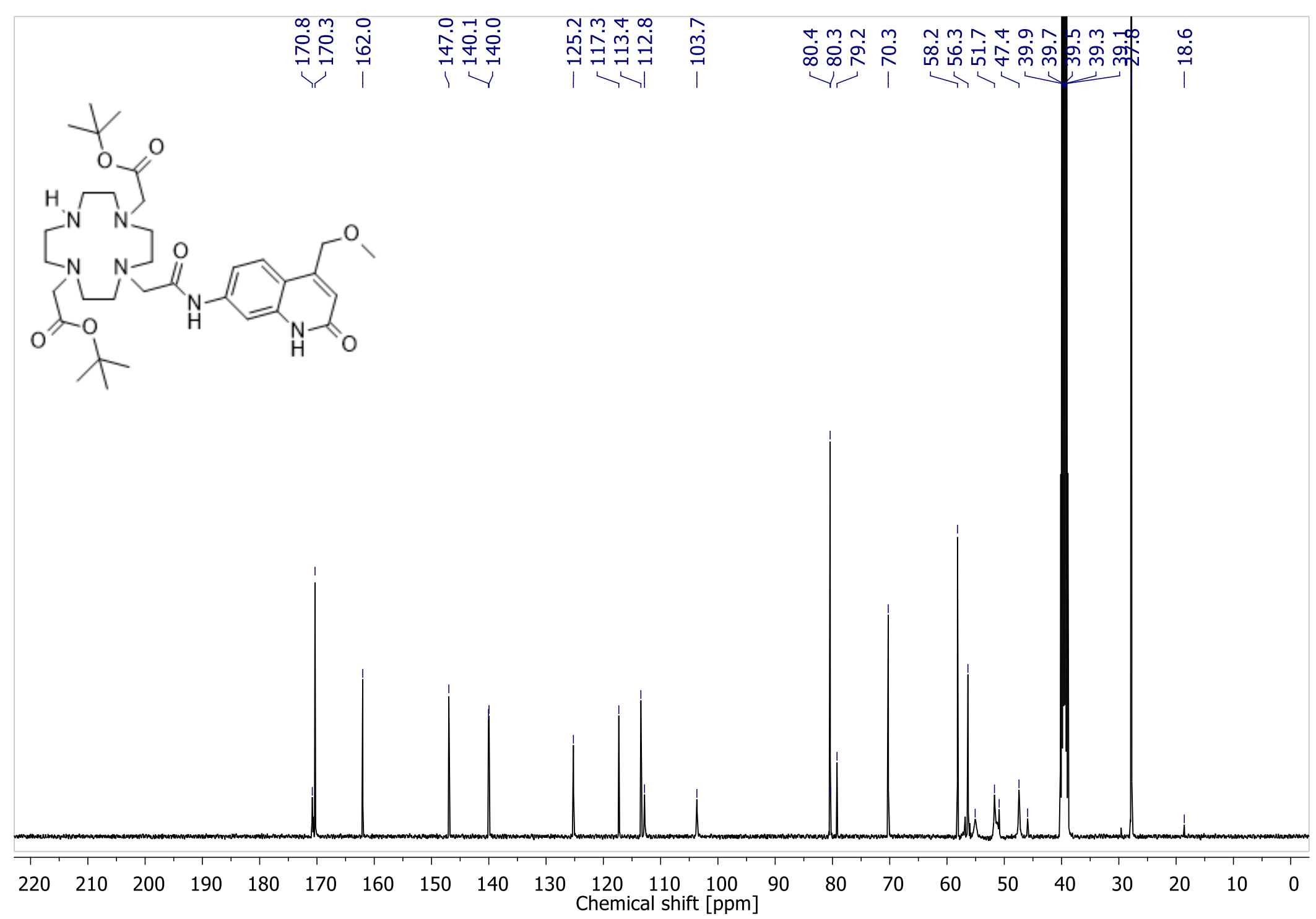

Figure S75. ${ }^{13} \mathrm{C}$ NMR spectrum of $3\left(101 \mathrm{MHz}, \mathrm{DMSO}-d_{6}\right)$. 


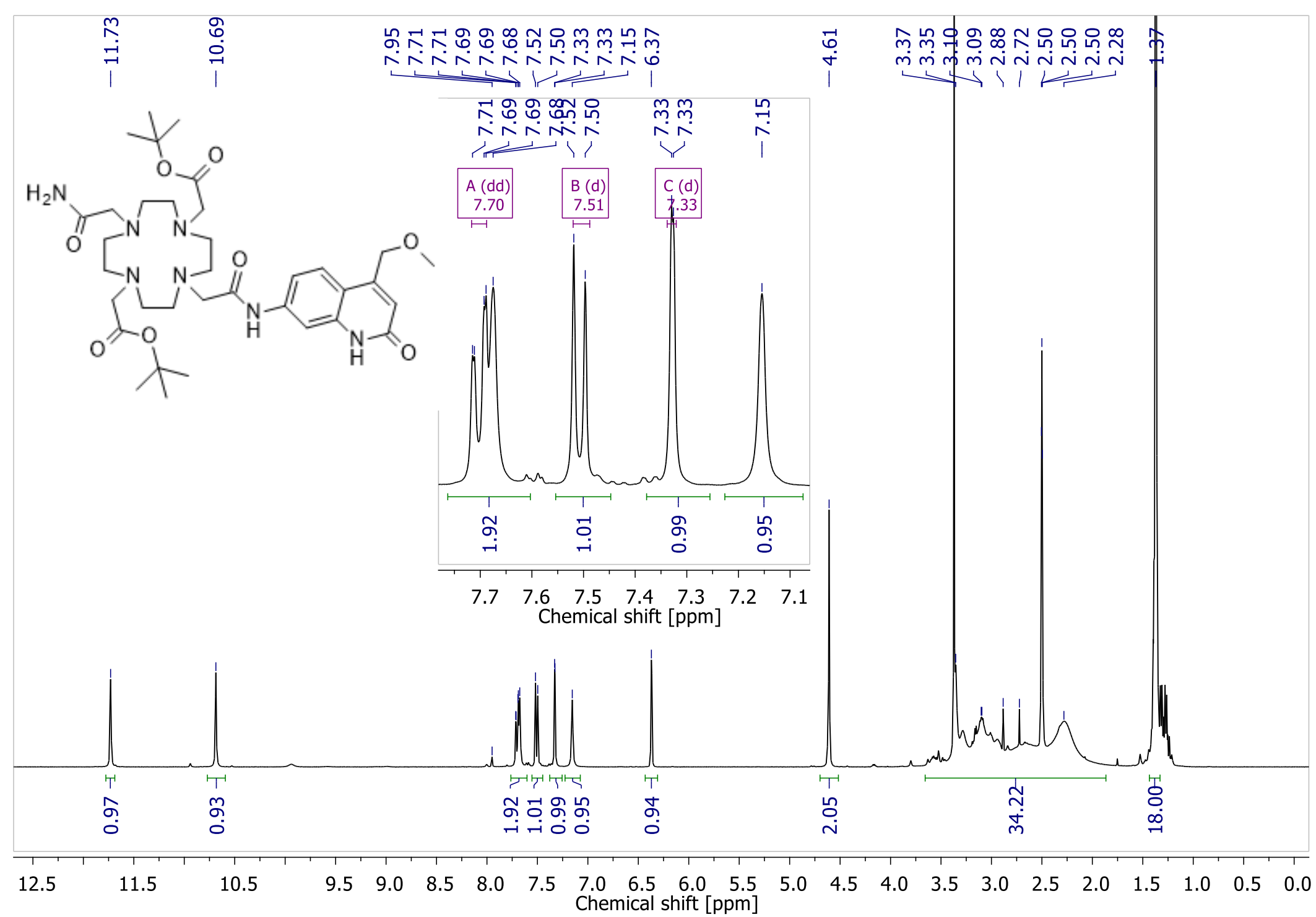

Figure S78. ${ }^{1} \mathrm{H}$ NMR spectrum of 5 (400 MHz, DMSO- $\left.d_{6}\right)$. 


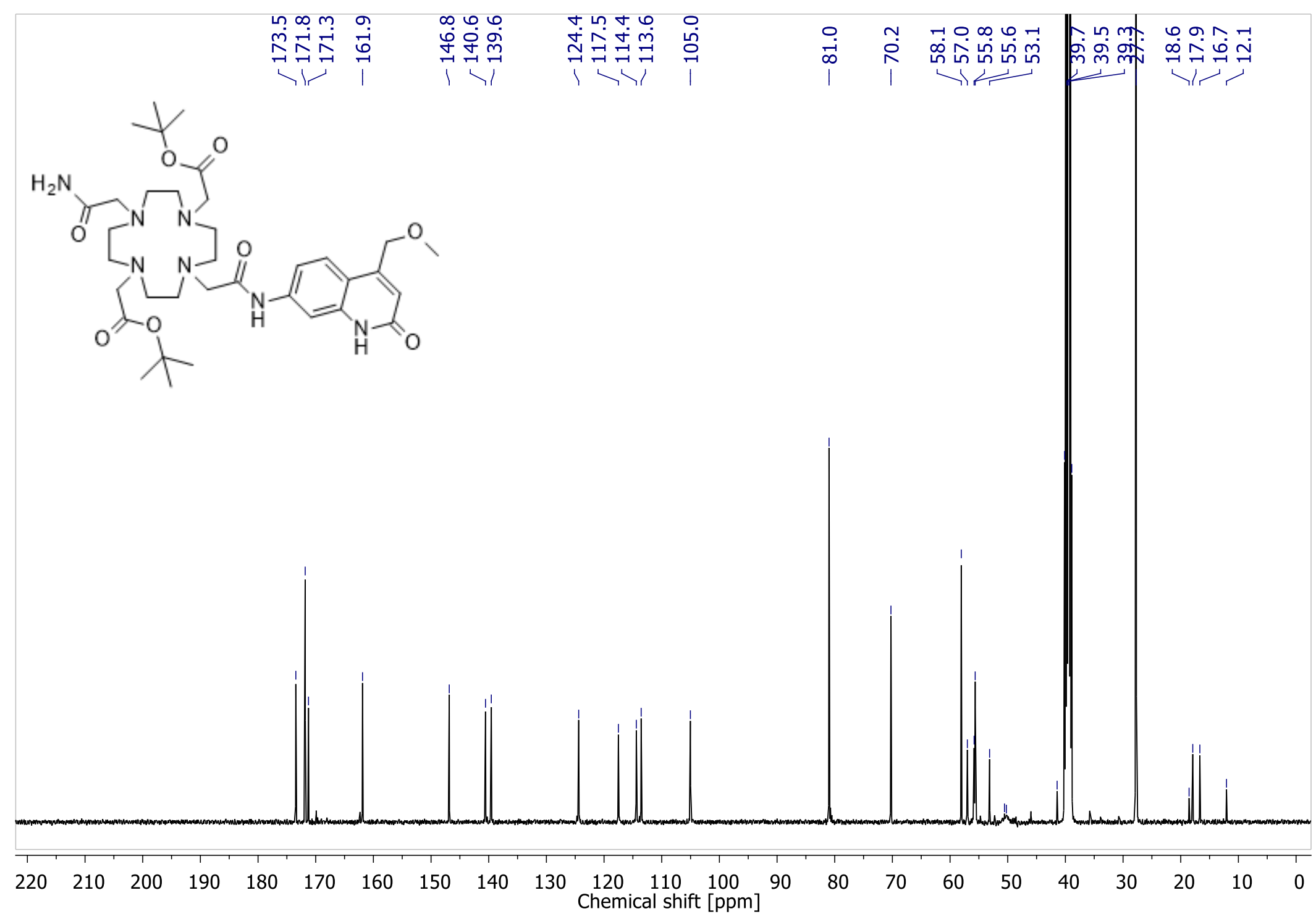

Figure S79. ${ }^{13} \mathrm{C}$ NMR spectrum of 5 (101 MHz, DMSO- $\left.d_{6}\right)$. 


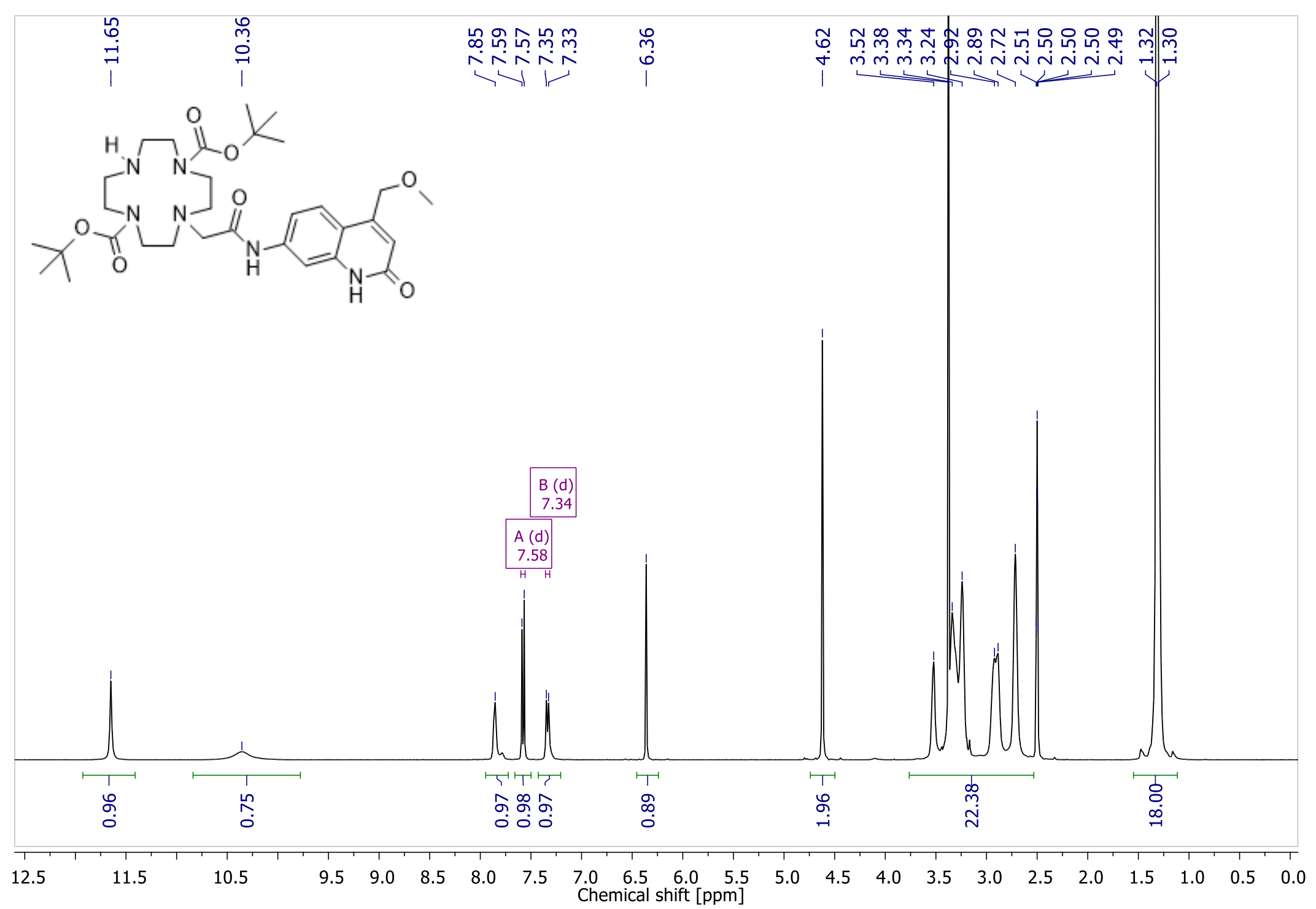

Figure S80. ${ }^{1} \mathrm{H}$ NMR spectrum of 7 (400 MHz, DMSO- $d_{6}$ ). 


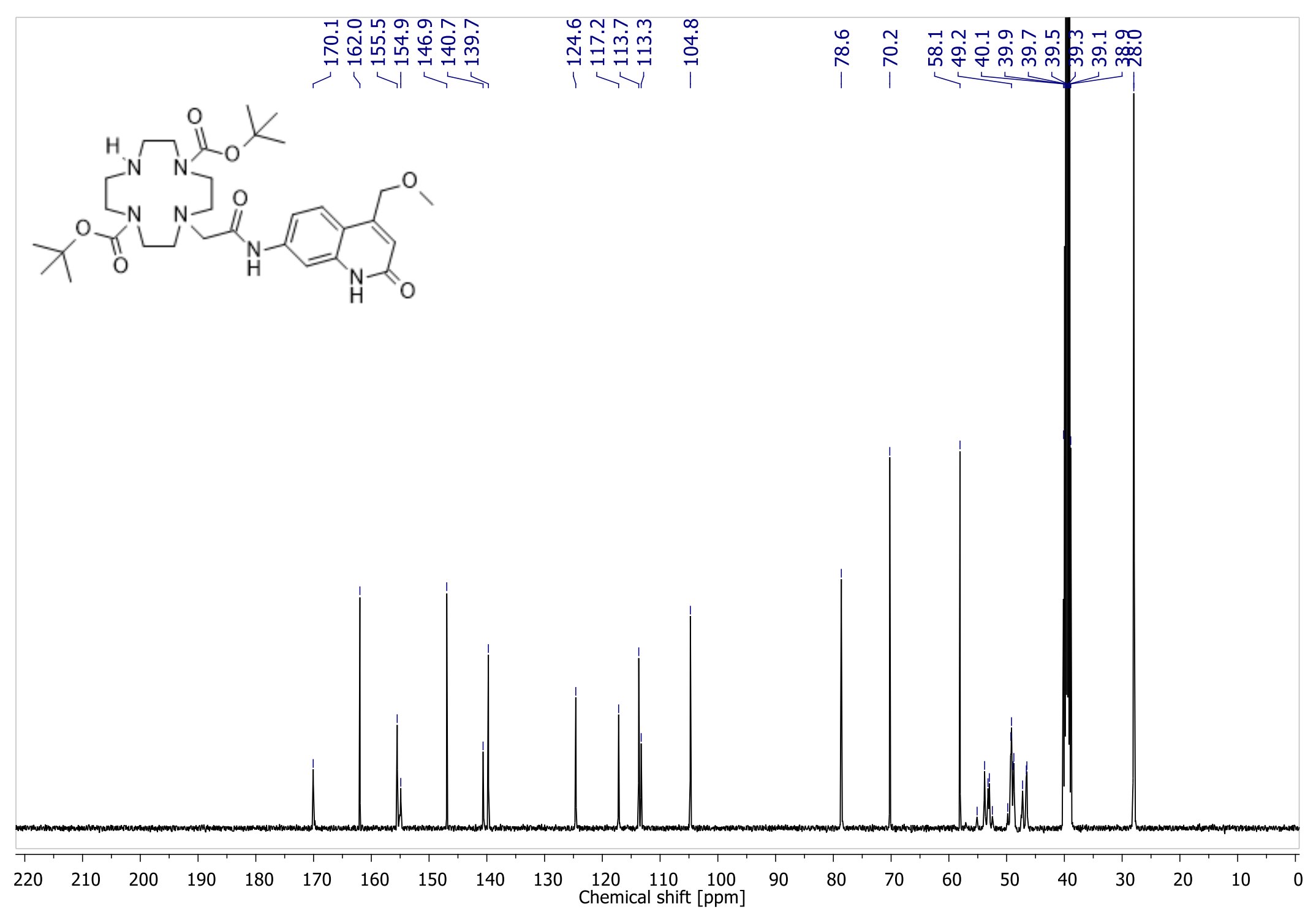

Figure S81. ${ }^{13} \mathrm{C}$ NMR spectrum of 7 (101 MHz, DMSO- $\left.d_{6}\right)$. 


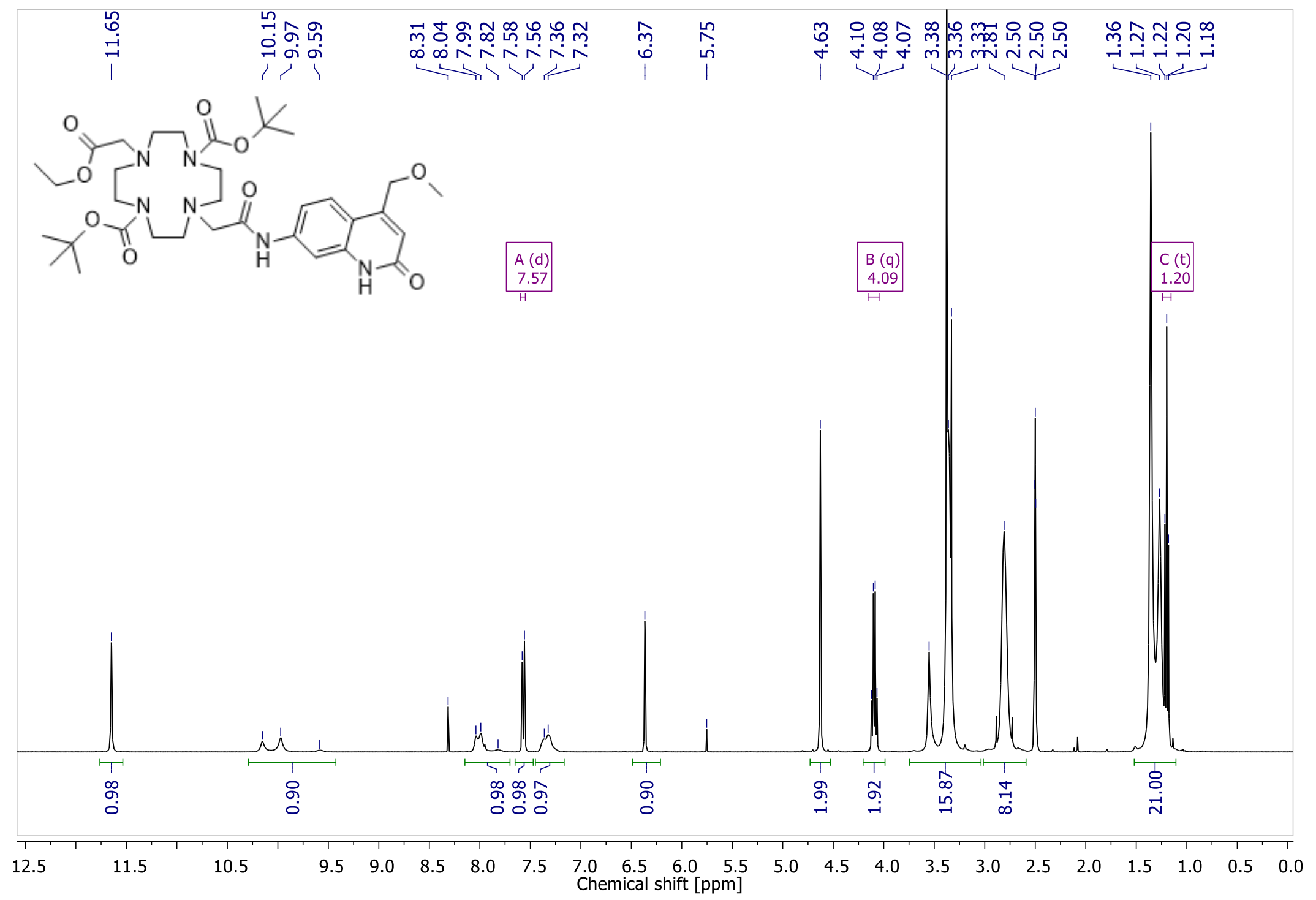

Figure S82. ${ }^{1} \mathrm{H}$ NMR spectrum of 8 (400 MHz, DMSO- $\left.d_{6}\right)$. 


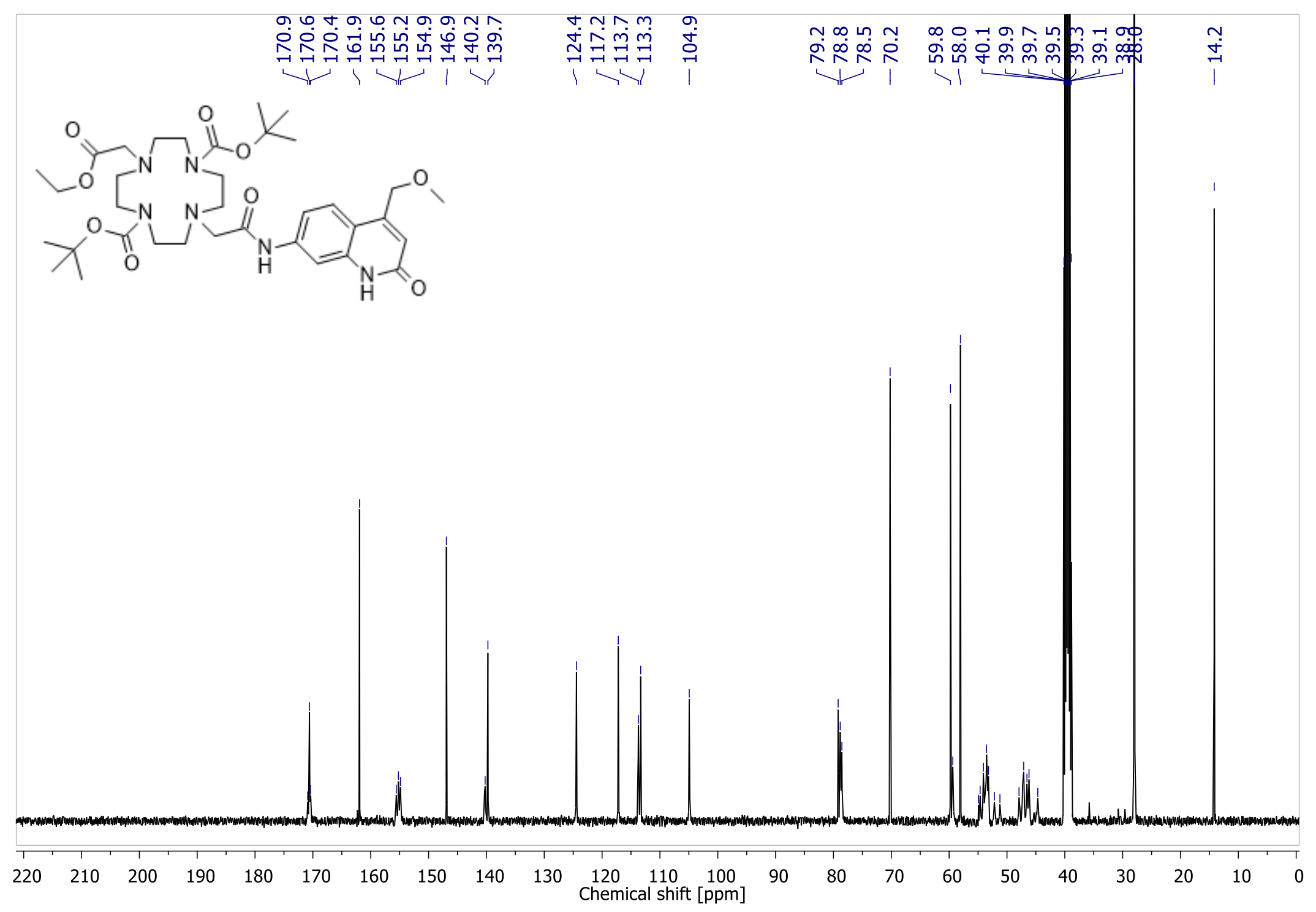

Figure S83. ${ }^{13} \mathrm{C}$ NMR spectrum of 8 (101 MHz, DMSO- $\left.d_{6}\right)$. 


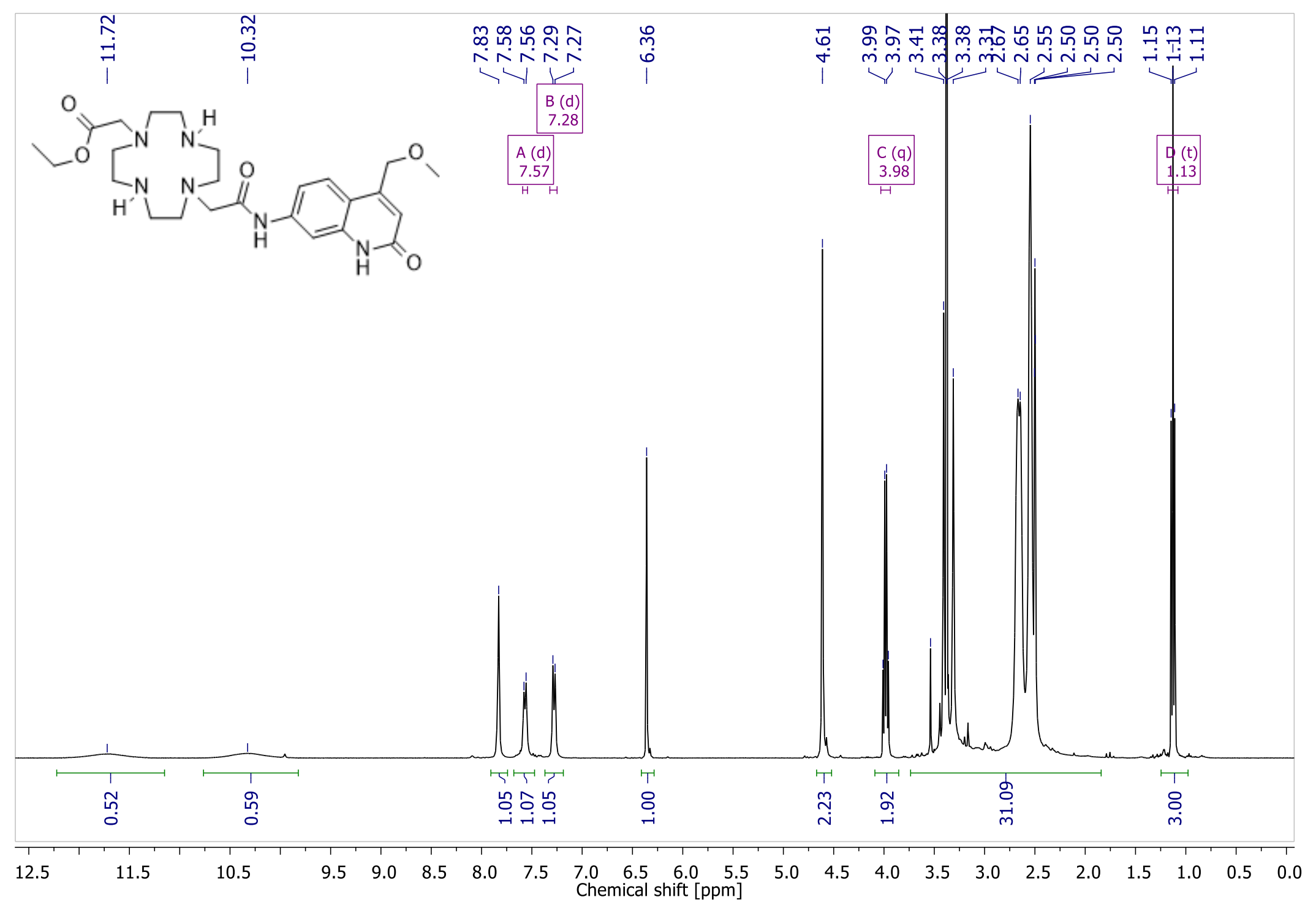

Figure S76. ${ }^{1} \mathrm{H}$ NMR spectrum of 9 (400 MHz, DMSO- $\left.d_{6}\right)$. 


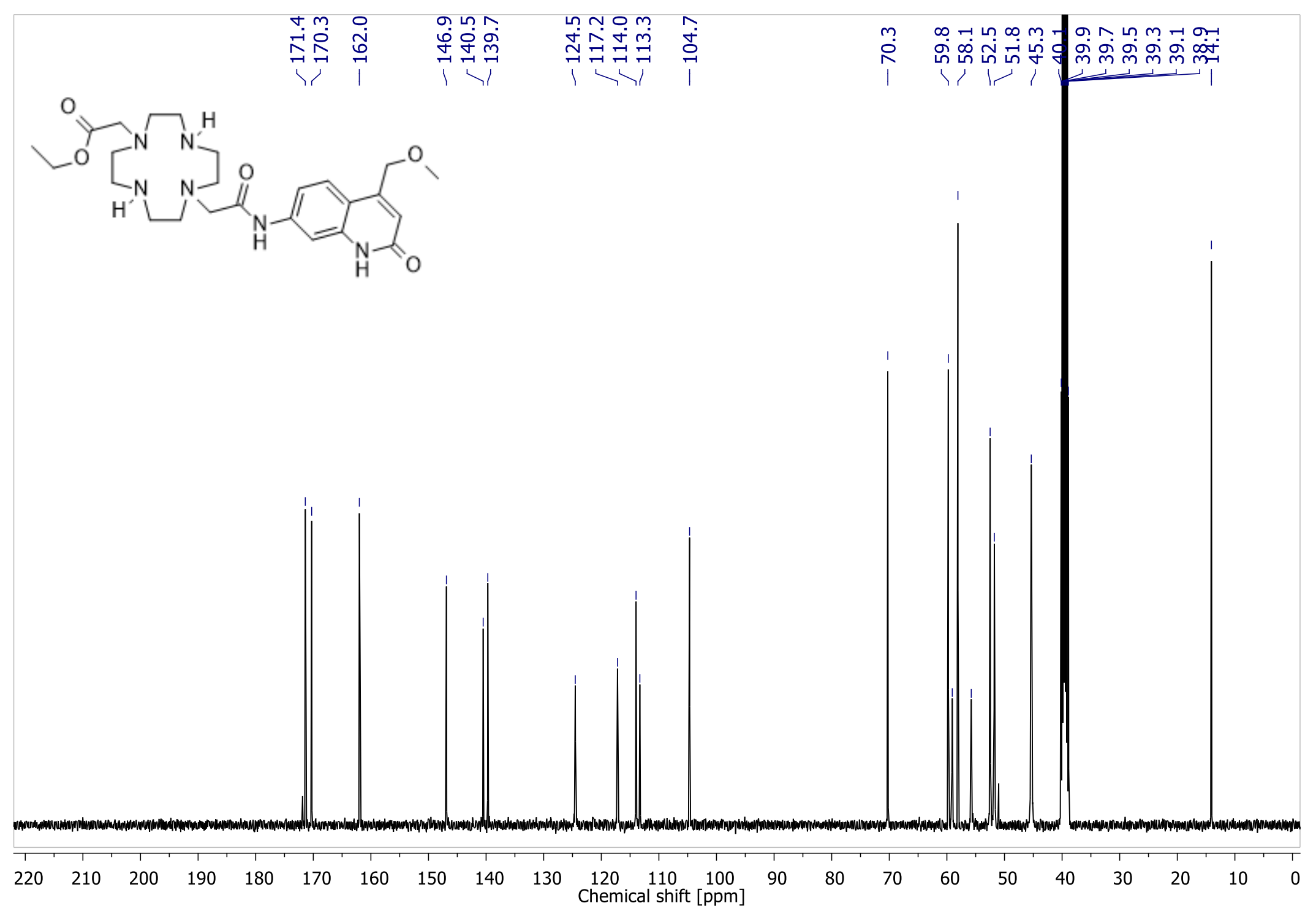

Figure S77. ${ }^{13} \mathrm{C}$ NMR spectrum of 9 (101 MHz, DMSO- $\left.d_{6}\right)$. 


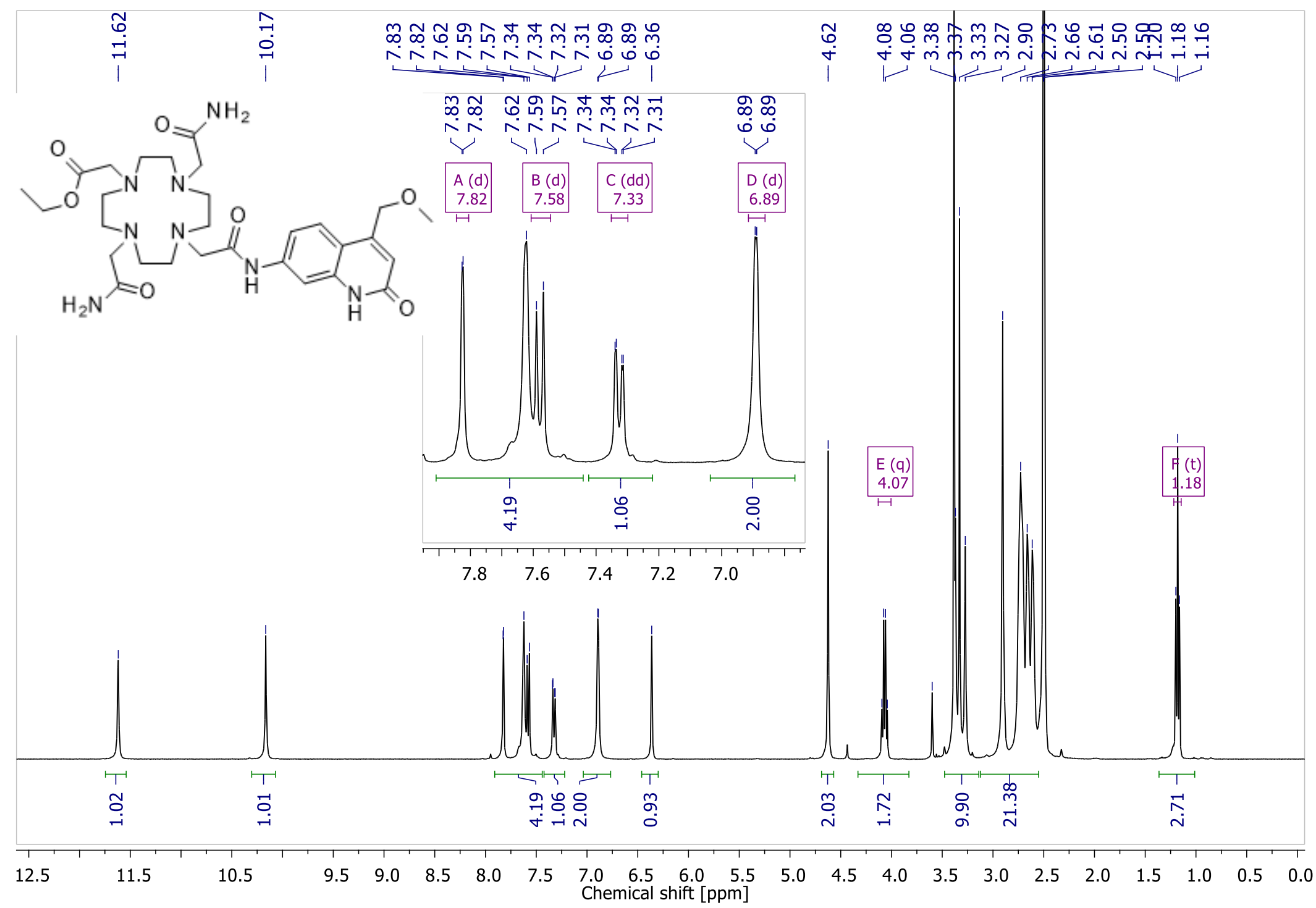

Figure S84. ${ }^{1} \mathrm{H}$ NMR spectrum of 10 (400 MHz, DMSO- $\left.d_{6}\right)$. 


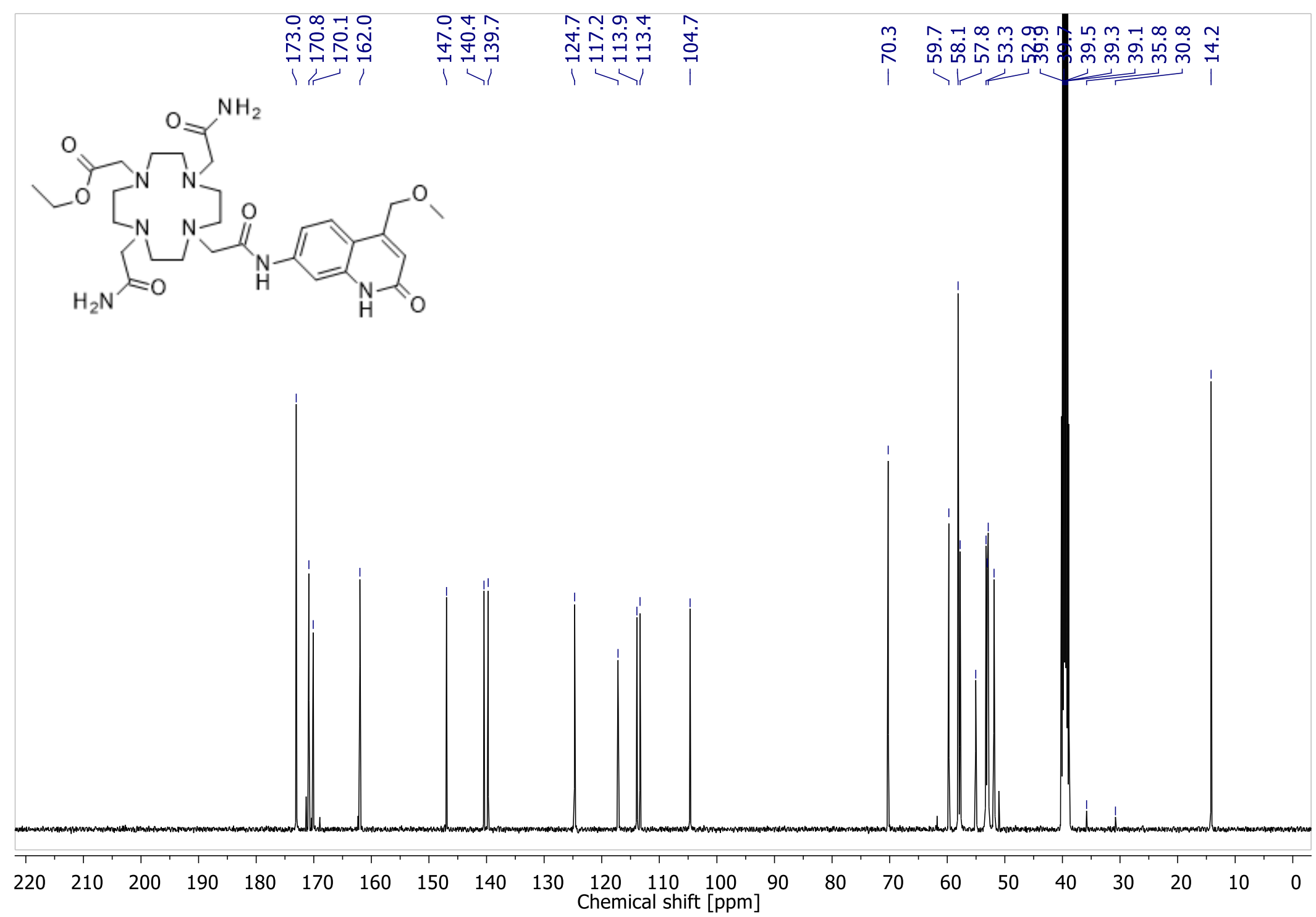

Figure S85. ${ }^{13} \mathrm{C}$ NMR spectrum of $\mathbf{1 0}\left(101 \mathrm{MHz}, \mathrm{DMSO}-d_{6}\right)$. 


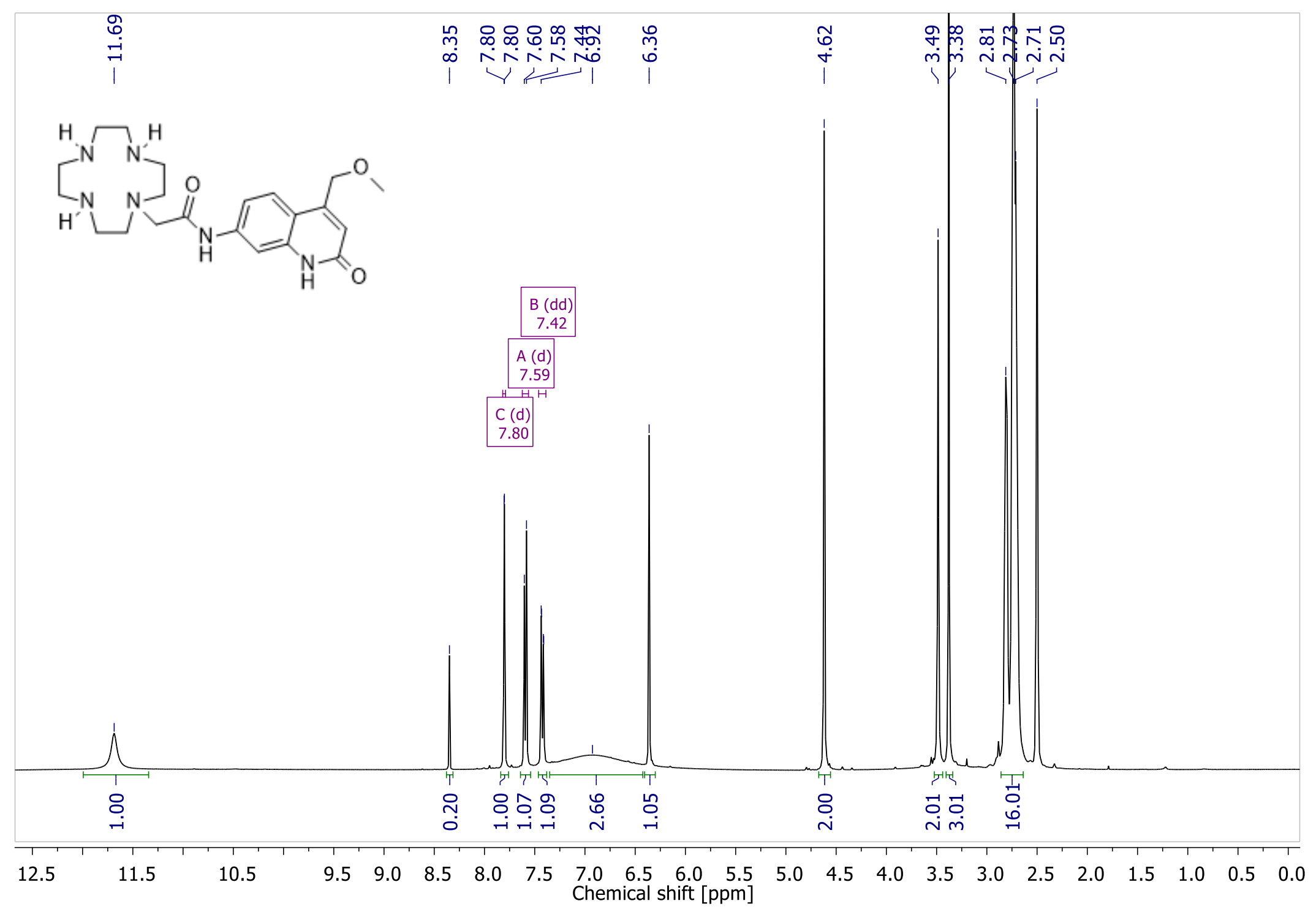

Figure S86. ${ }^{1} \mathrm{H}$ NMR spectrum of $\mathbf{1 1}^{\mathrm{MOM}}\left(400 \mathrm{MHz}, \mathrm{DMSO}-d_{6}\right)$. 


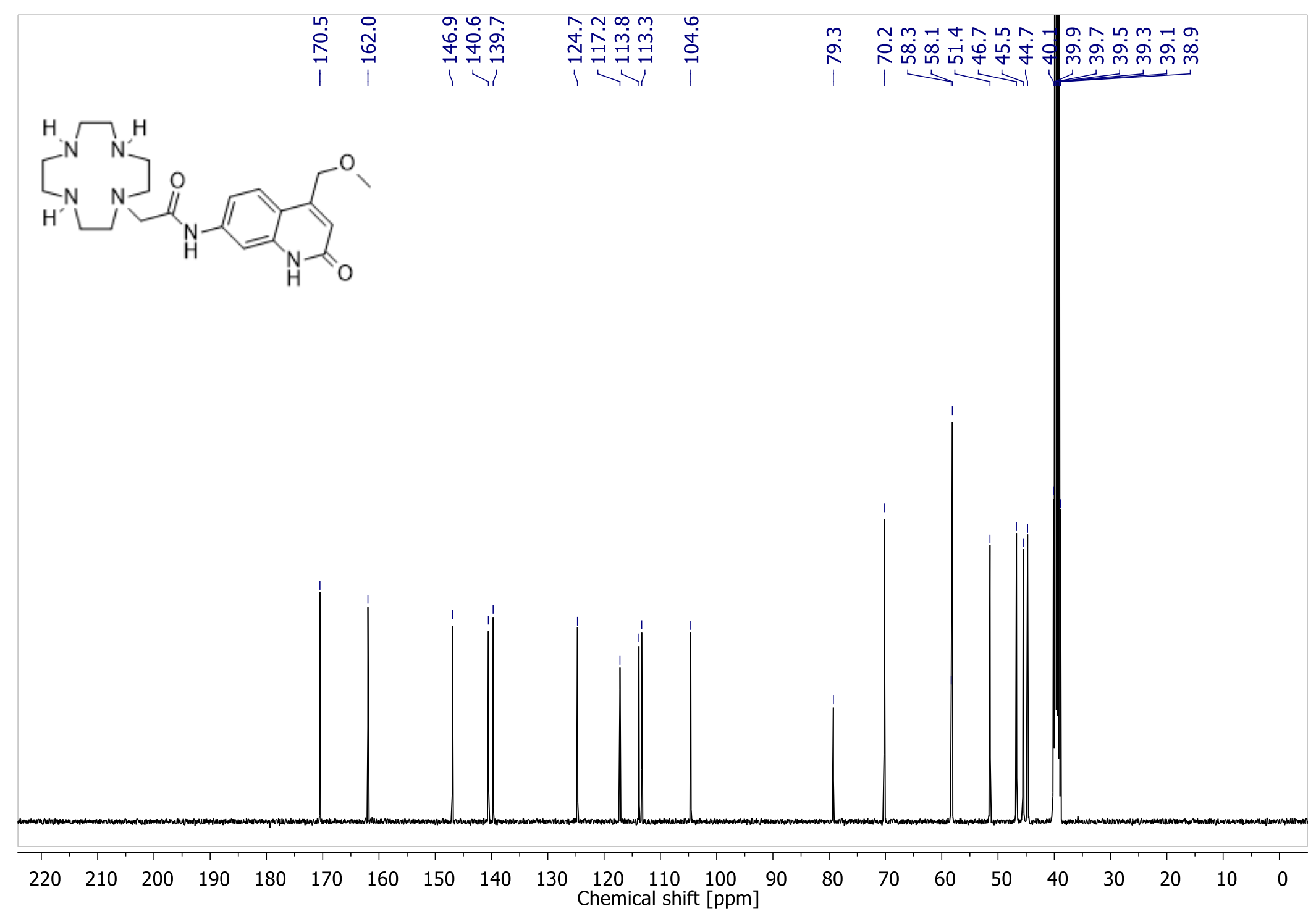

Figure S87. ${ }^{13} \mathrm{C}$ NMR spectrum of $\mathbf{1 1}^{\text {Mом }}$ (101 MHz, DMSO- $\left.d_{6}\right)$. 


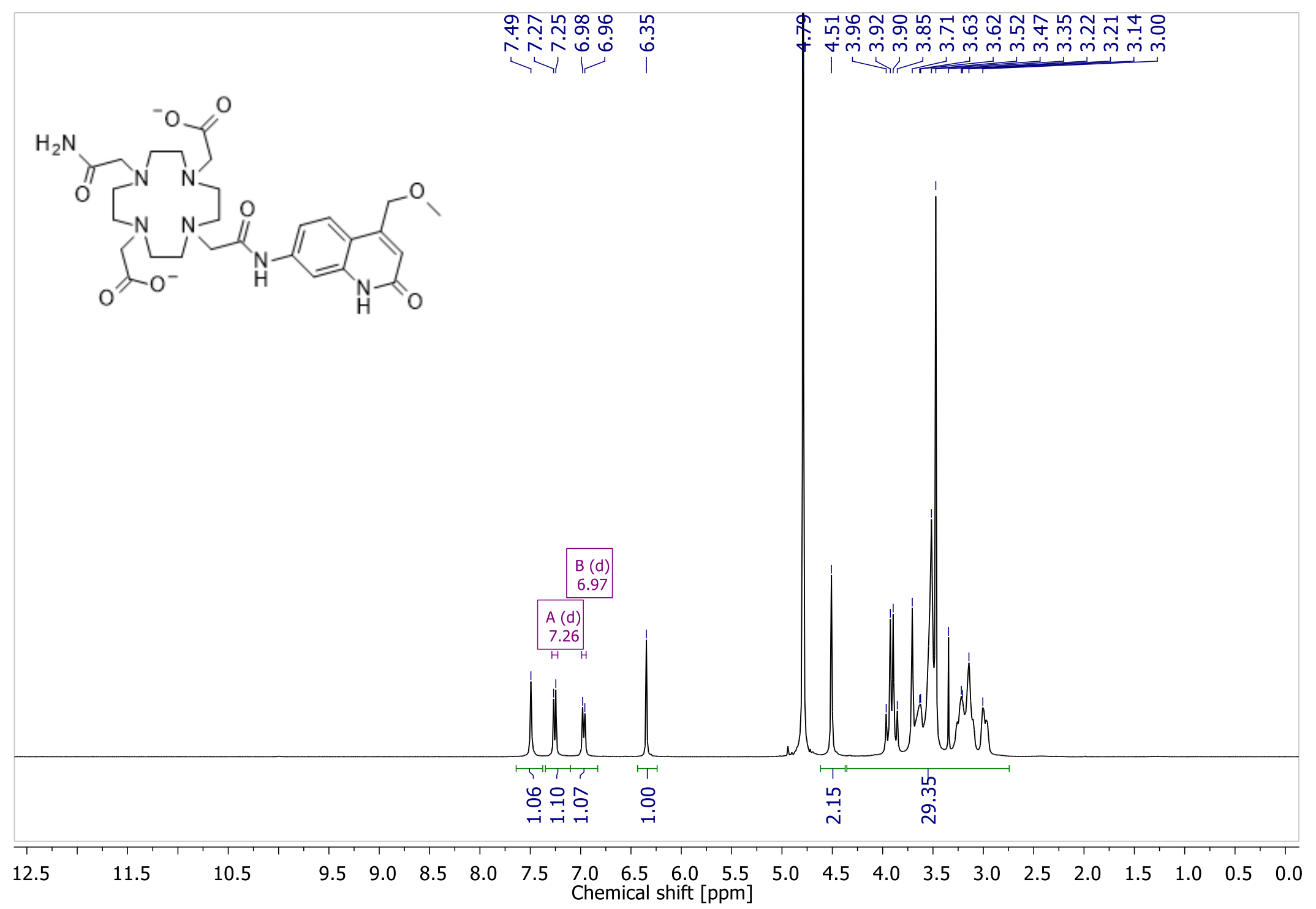

Figure S88. ${ }^{1} \mathrm{H}$ NMR spectrum of $\mathbf{L 2}{ }^{\text {MOM }}$ (400 MHz, DMSO- $d_{6}$ ). 


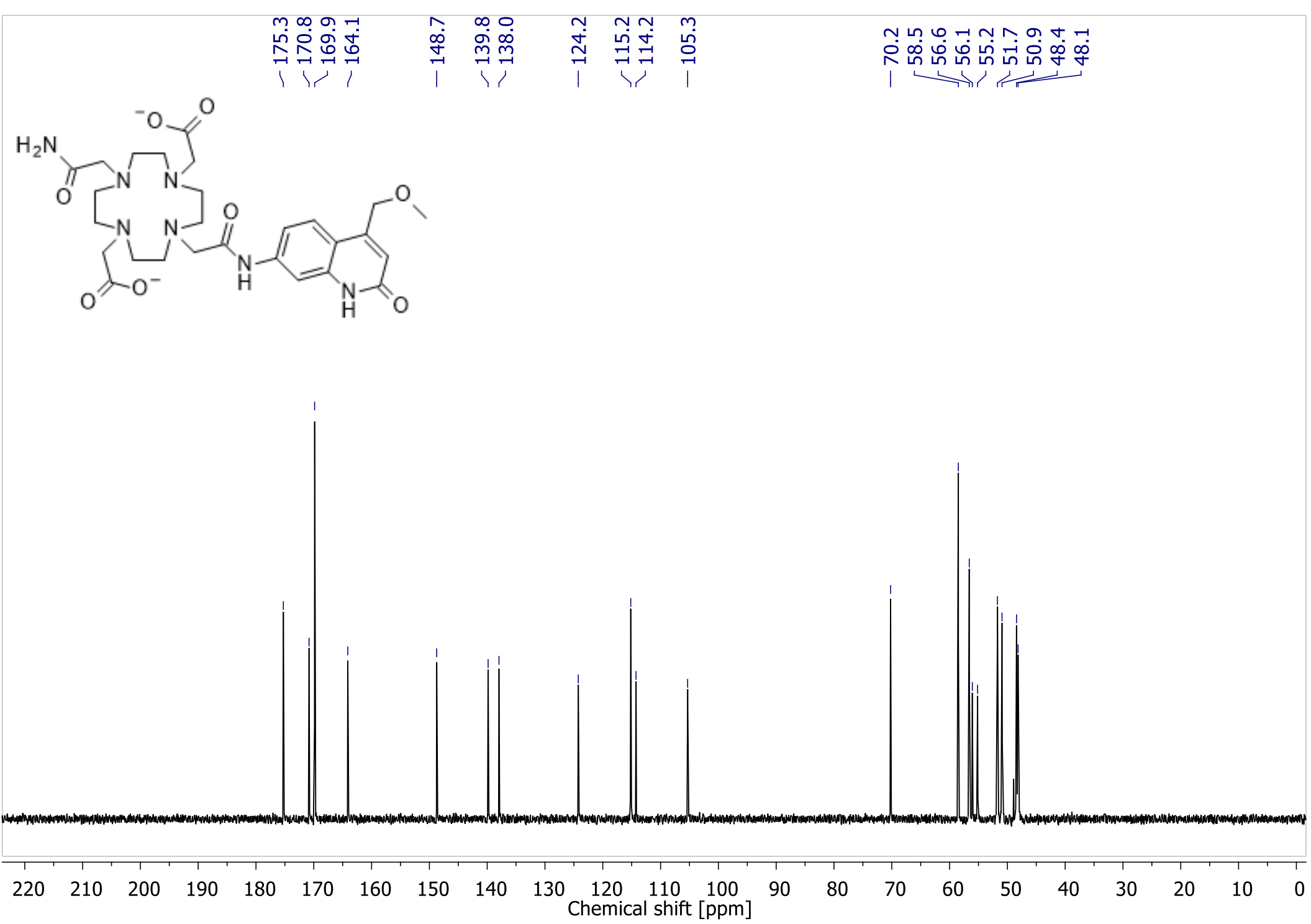

Figure S89. ${ }^{13} \mathrm{C}$ NMR spectrum of $\mathbf{L} \mathbf{2}^{\text {MOM }}$ (101 MHz, DMSO- $\left.d_{6}\right)$. 


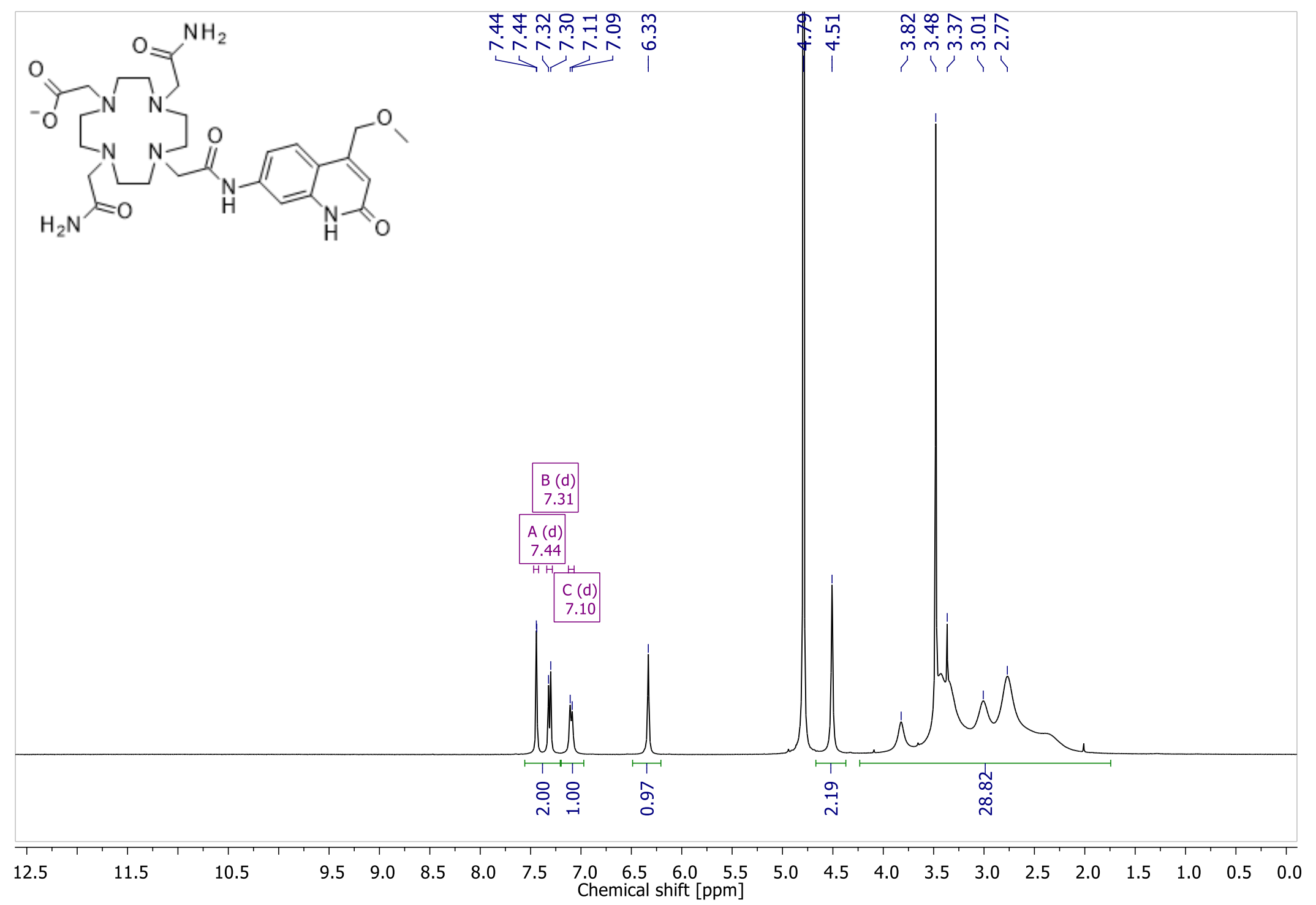

Figure S90. ${ }^{1} \mathrm{H}$ NMR spectrum of $\mathbf{L 3}^{\text {MOM }}$ (400 MHz, DMSO- $d_{6}$ ). 


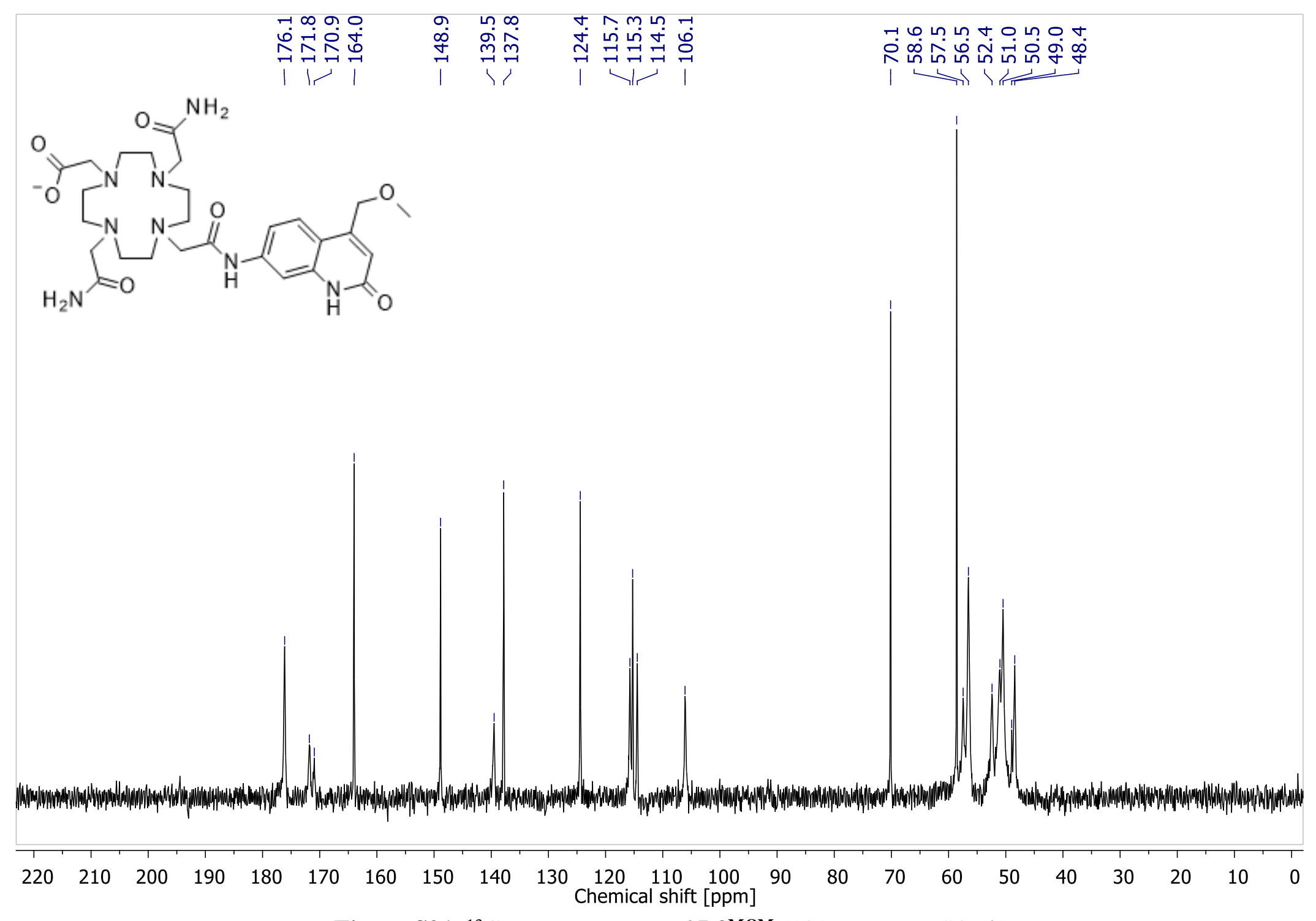

Figure S91. ${ }^{13} \mathrm{C}$ NMR spectrum of $\mathbf{L 3}^{\mathrm{MOM}}$ (101 MHz, DMSO-d $d_{6}$ ). 


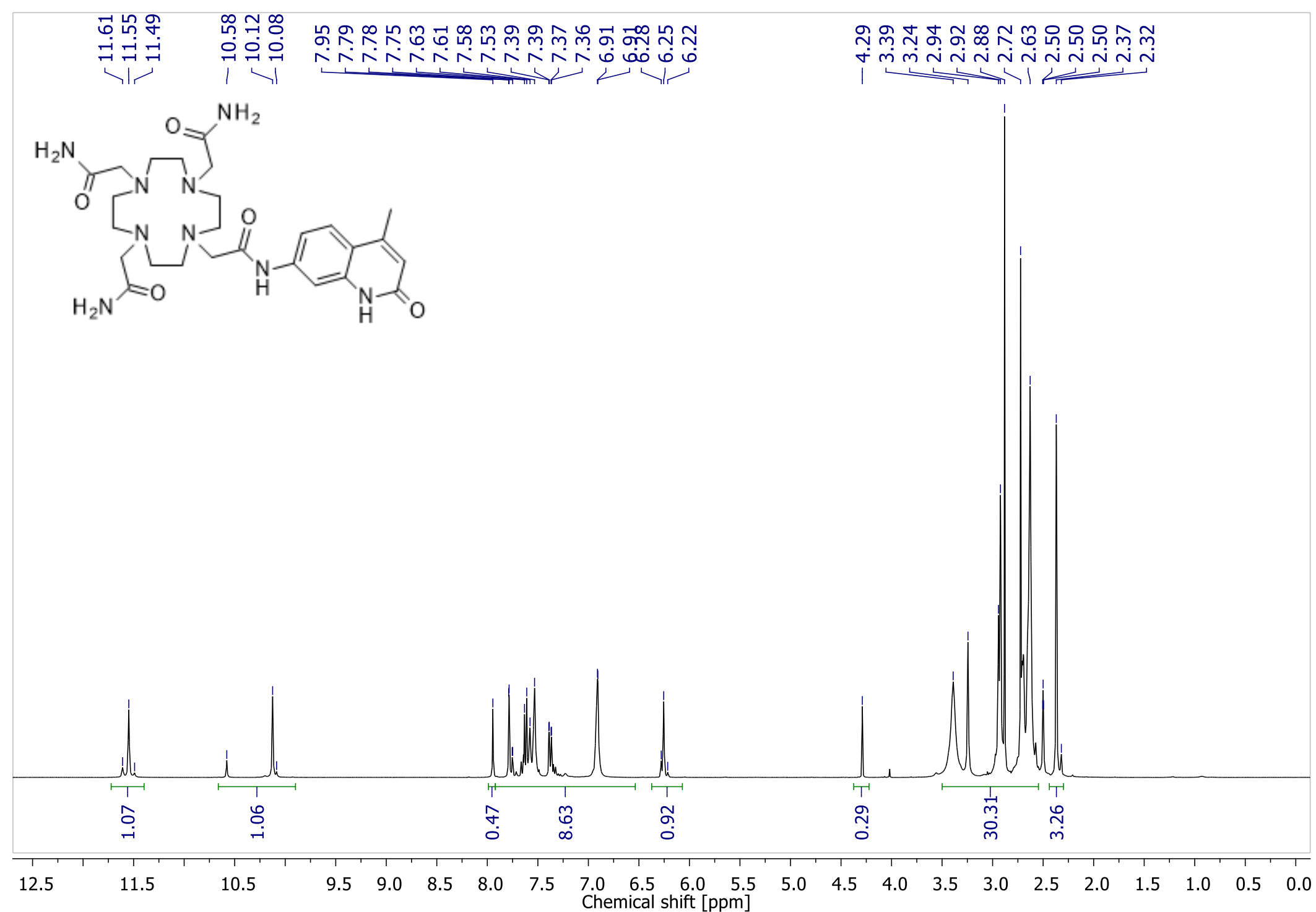

Figure S92. ${ }^{1} \mathrm{H}$ NMR spectrum of $\mathbf{L 4 a ^ { M e }}\left(400 \mathrm{MHz}\right.$, DMSO- $\left.d_{6}\right)$. 


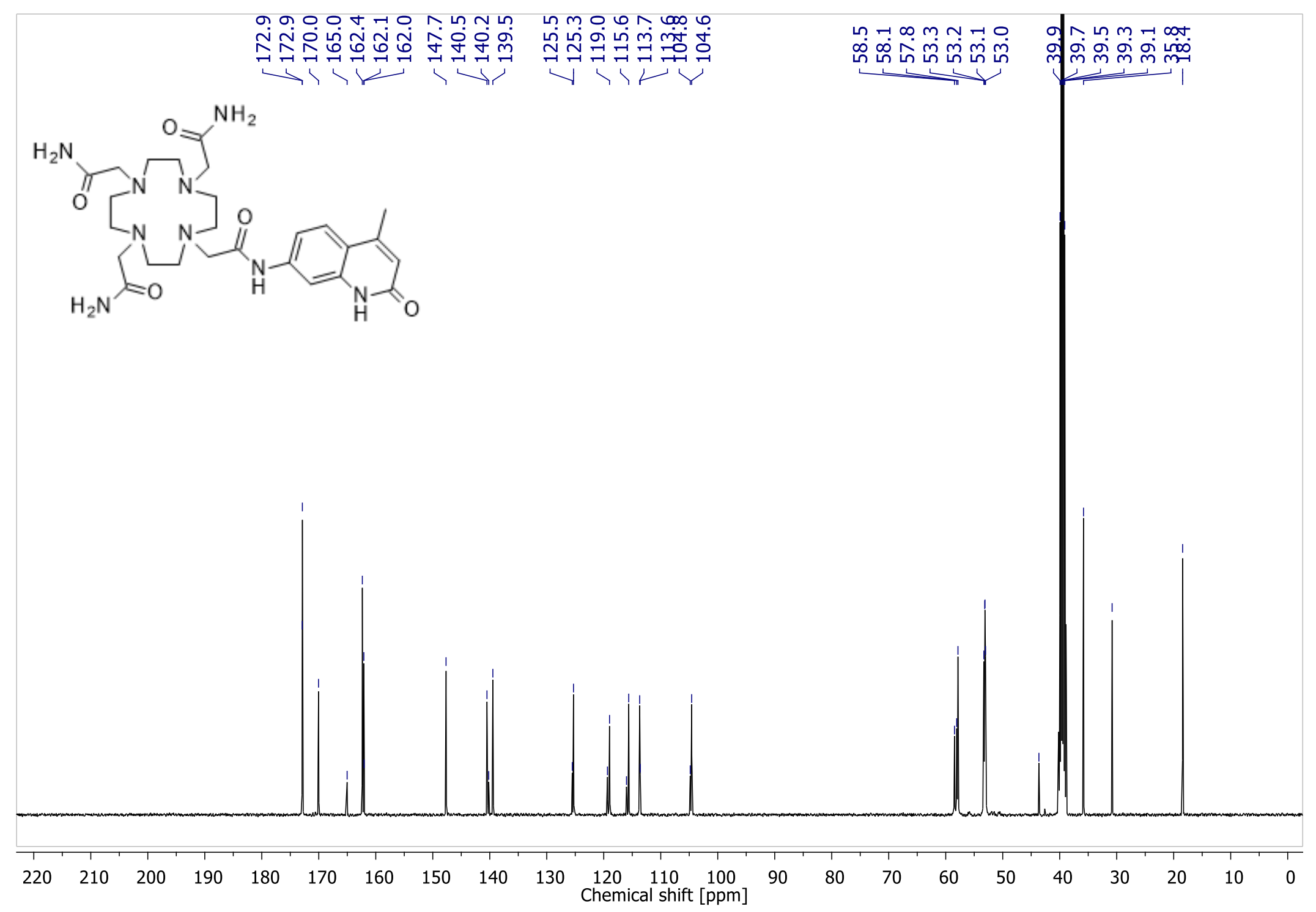

Figure S93. ${ }^{13} \mathrm{C}$ NMR spectrum of $\mathbf{L} 4 \mathbf{a}^{\mathrm{Me}}\left(101 \mathrm{MHz}\right.$, DMSO- $\left.d_{6}\right)$. 


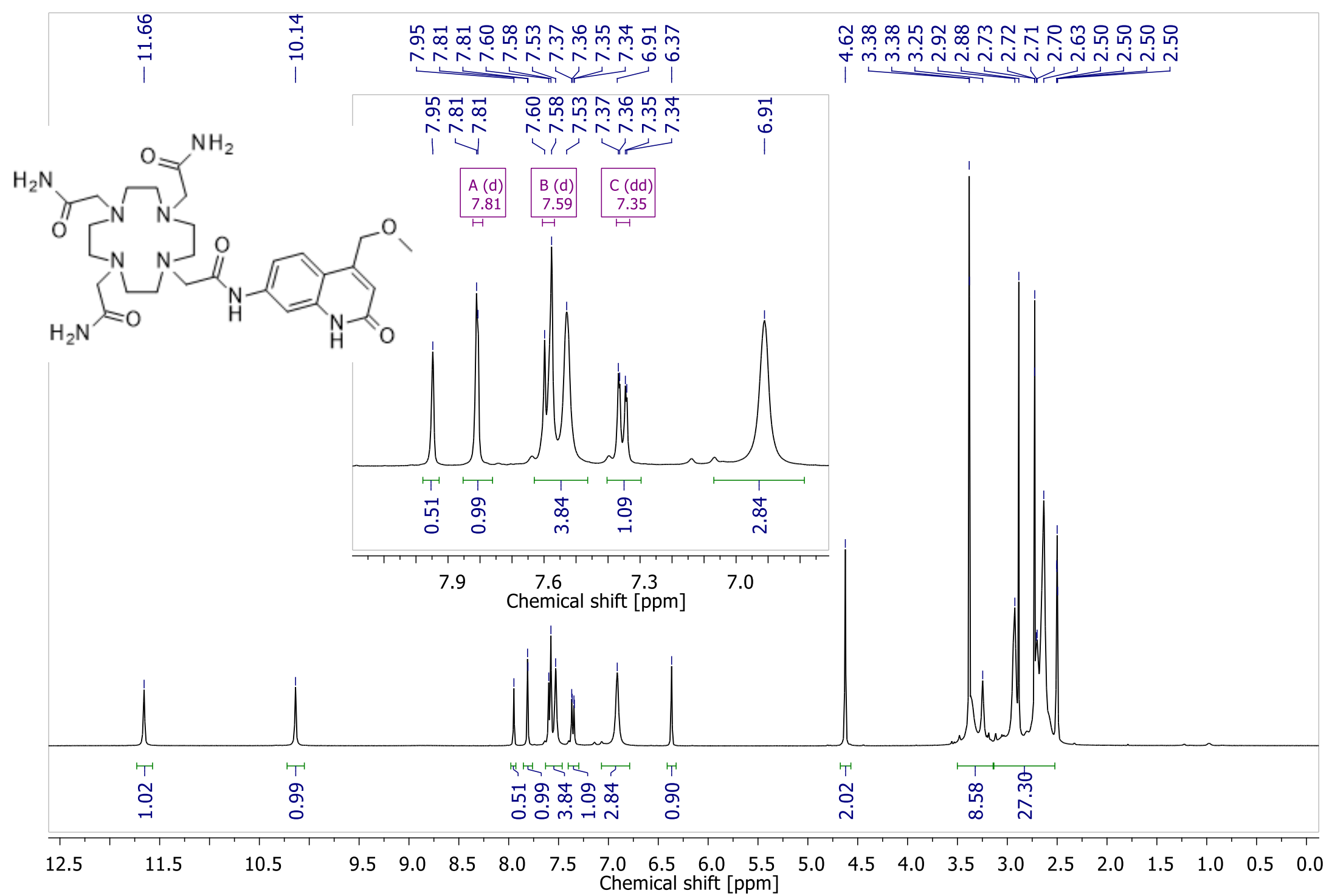

Figure S94. ${ }^{1} \mathrm{H}$ NMR spectrum of $\mathbf{L 4 \mathbf { a } ^ { \text { MOM } }}$ (400 MHz, DMSO- $d_{6}$ ). 


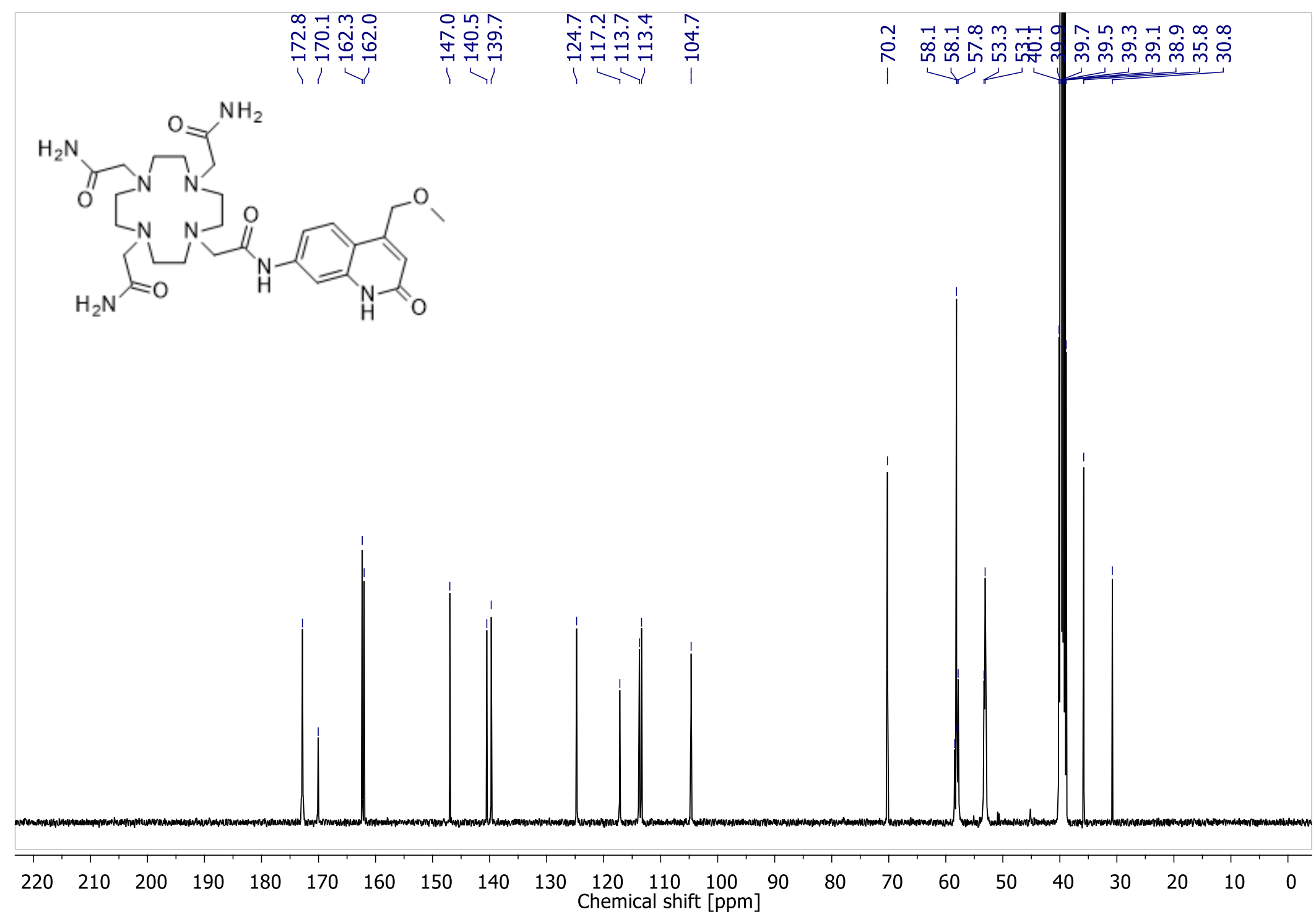

Figure S95. ${ }^{13} \mathrm{C}$ NMR spectrum of $\mathbf{L 4 a ^ { \text { MOM } }}(101 \mathrm{MHz}$, DMSO-d 6 ). 


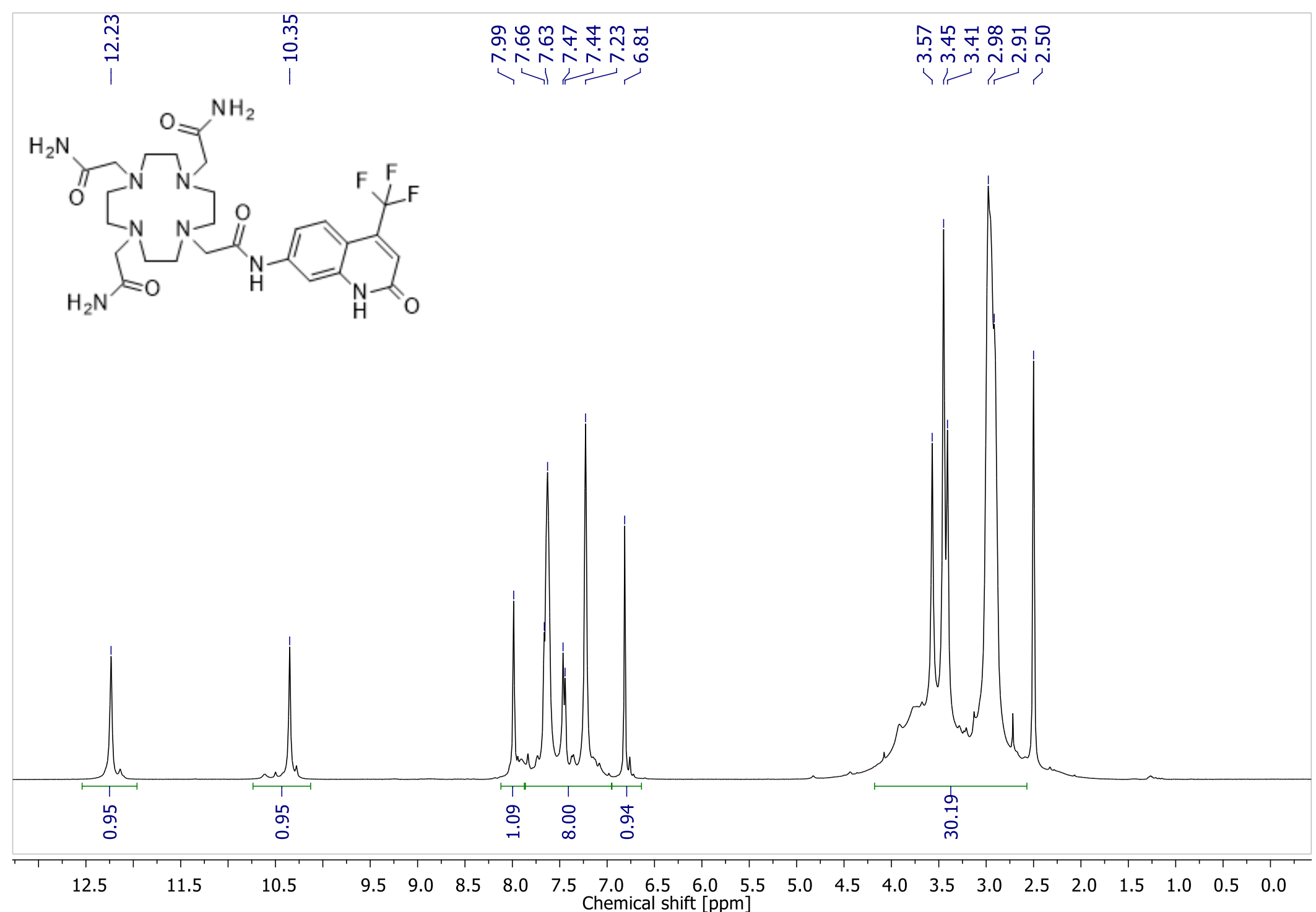

Figure S96. ${ }^{1} \mathrm{H}$ NMR spectrum of $\mathbf{L} \mathbf{4 a}^{\mathbf{C F 3}}\left(400 \mathrm{MHz}, \mathrm{DMSO}-d_{6}\right)$. 


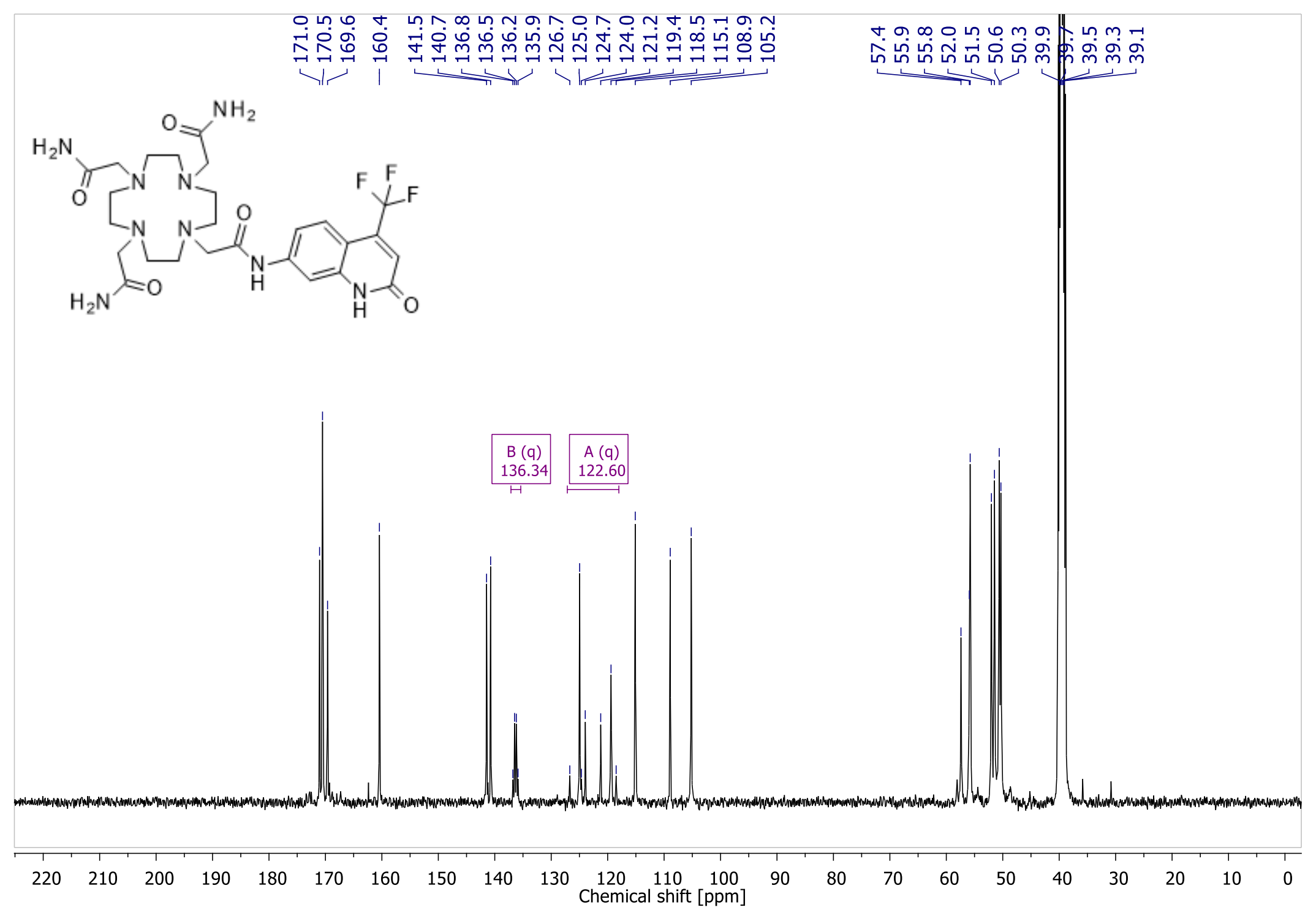

Figure S97. ${ }^{13} \mathrm{C}$ NMR spectrum of $\mathbf{L 4 a} \mathbf{a}^{\mathbf{C F 3}}$ (101 MHz, DMSO- $\left.d_{6}\right)$. 


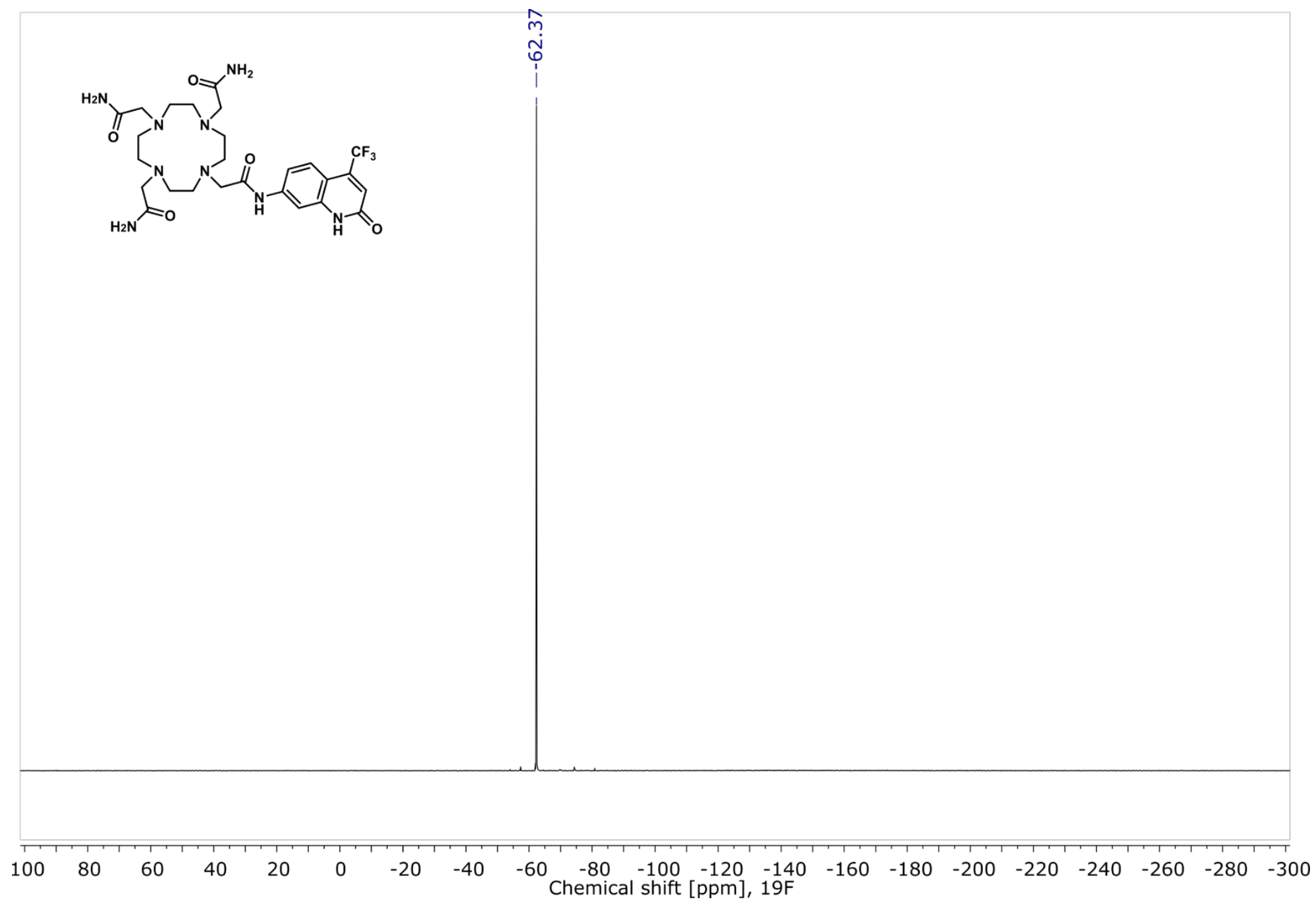

Figure S98. ${ }^{19} \mathrm{~F}$ NMR spectrum of $\mathbf{L 4 a ^ { \mathbf { C F 3 } }}$ (376 MHz, DMSO- $d_{6}$ ). 


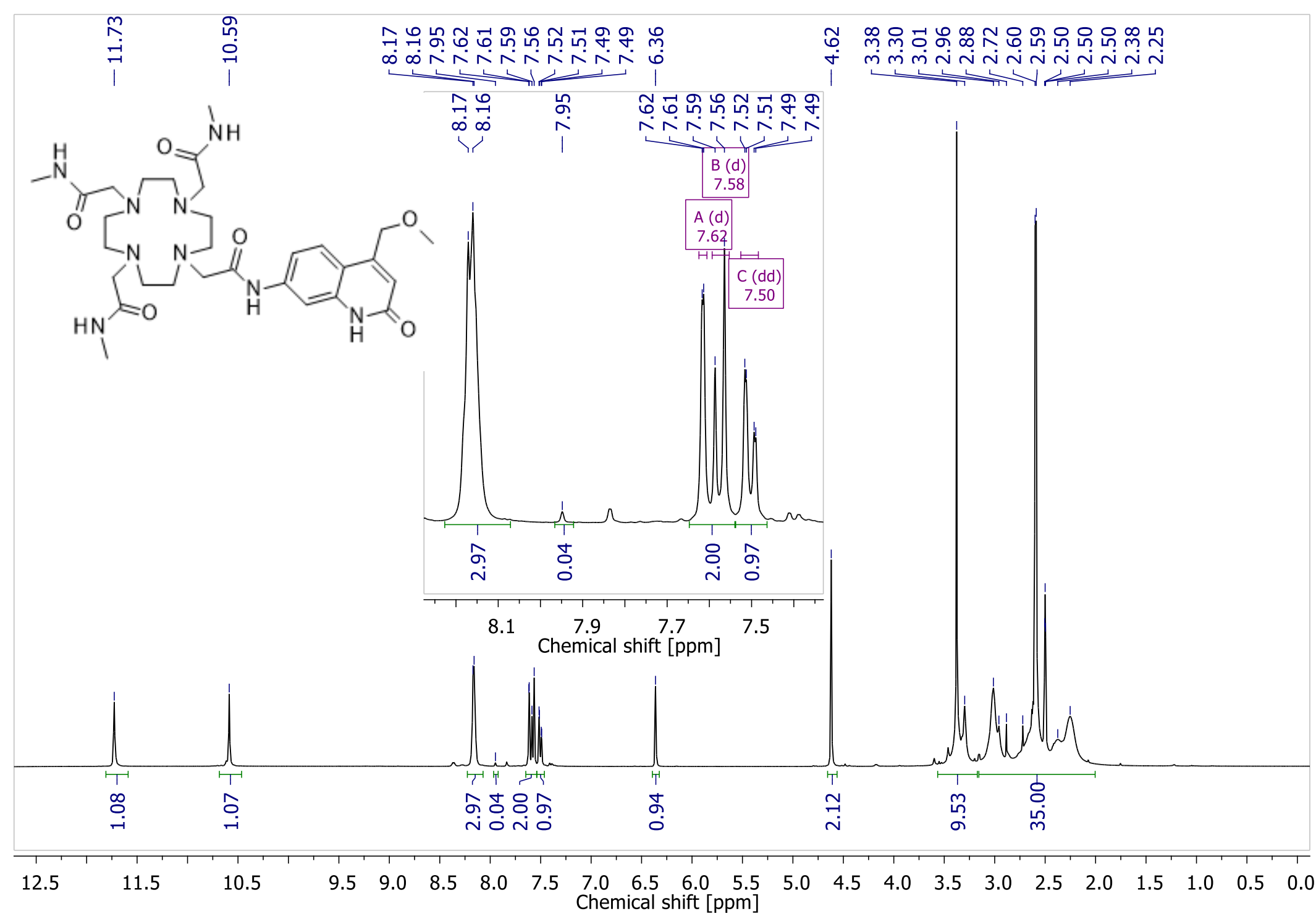

Figure S99. ${ }^{1} \mathrm{H}$ NMR spectrum of $\mathbf{L} 4 \mathbf{b}^{\text {MOM }}$ (400 MHz, DMSO- $d_{6}$ ). 


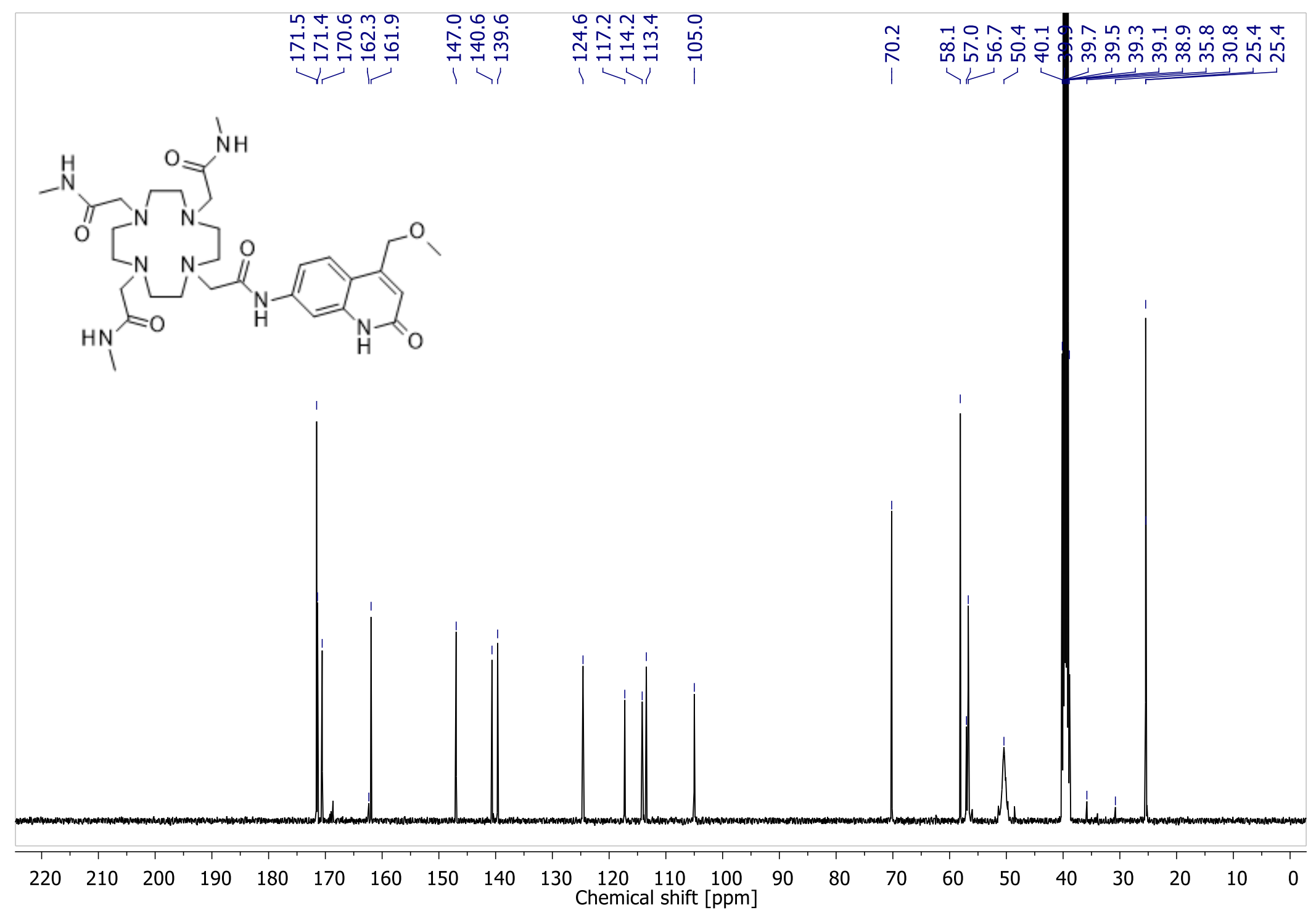

Figure S100. ${ }^{13} \mathrm{C}$ NMR spectrum of $\mathbf{L} 4 \mathbf{b}^{\text {MOM }}\left(101 \mathrm{MHz}, \mathrm{DMSO}-d_{6}\right)$. 


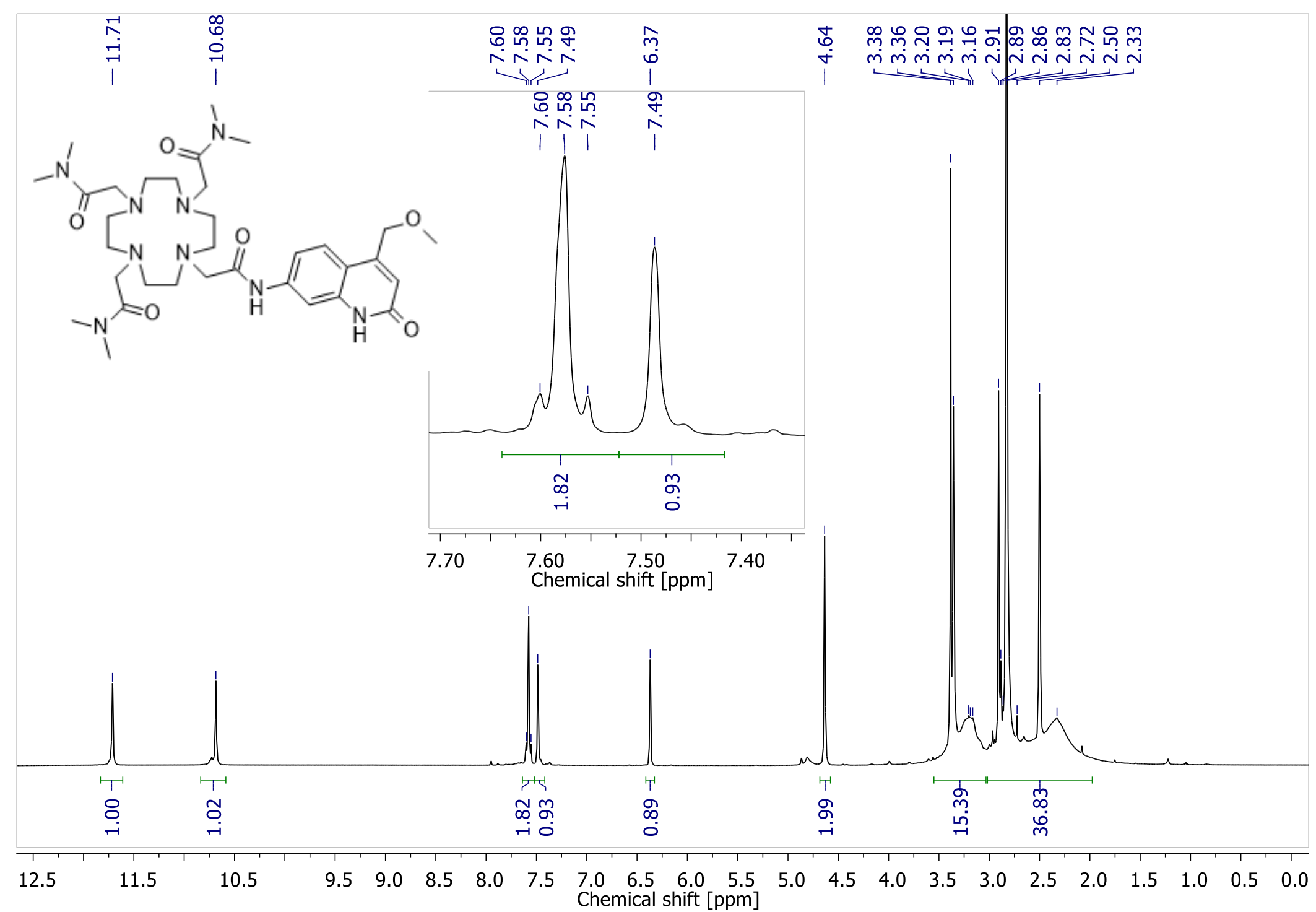

Figure S101. ${ }^{1} \mathrm{H}$ NMR spectrum of $\mathbf{L} 4 \mathbf{c}^{\mathrm{MOM}}\left(400 \mathrm{MHz}, \mathrm{DMSO}-d_{6}\right)$. 


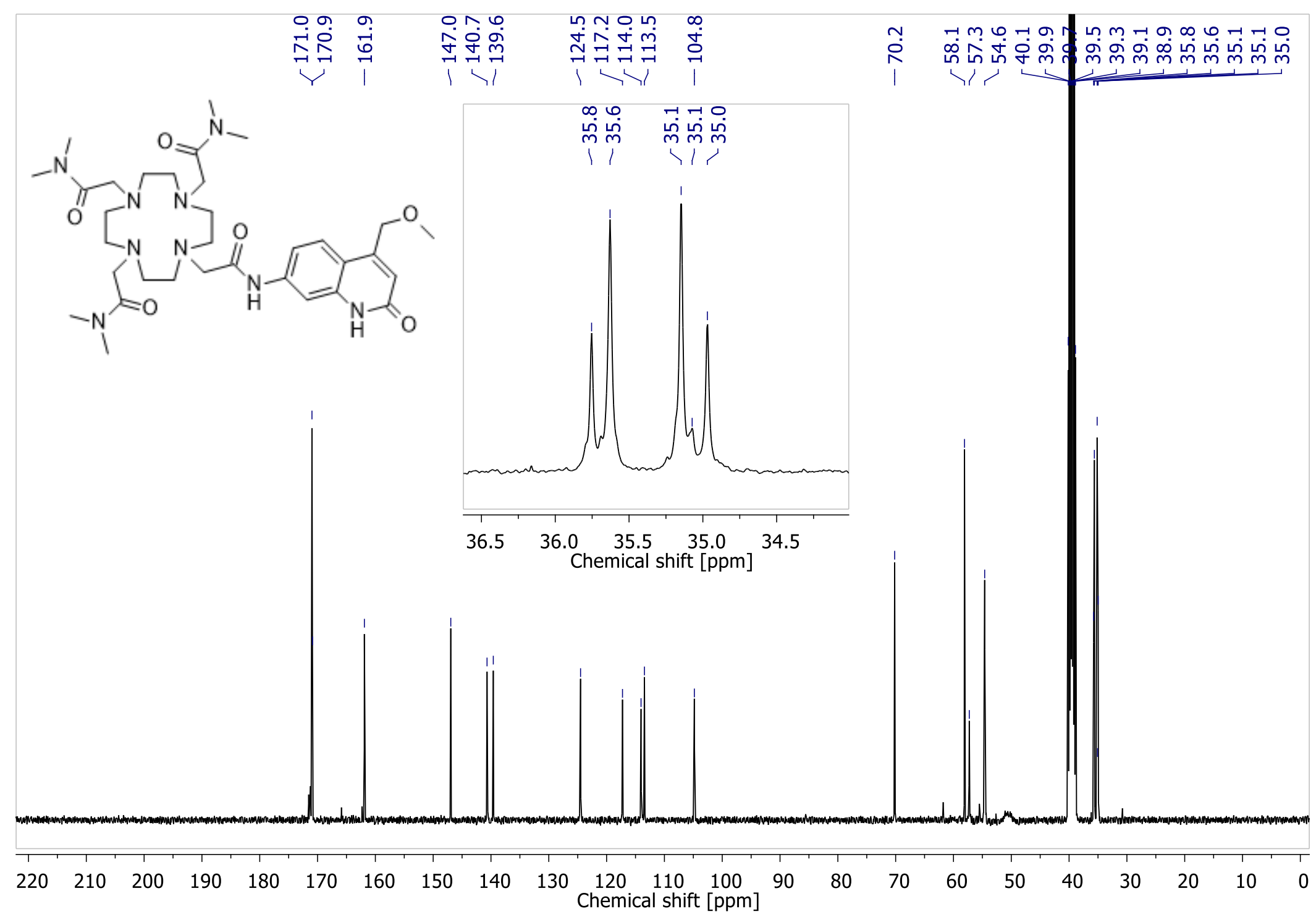

Figure S102. ${ }^{13} \mathrm{C}$ NMR spectrum of $\mathbf{L 4 c ^ { M O M }}\left(101 \mathrm{MHz}, \mathrm{DMSO}-d_{6}\right)$. 


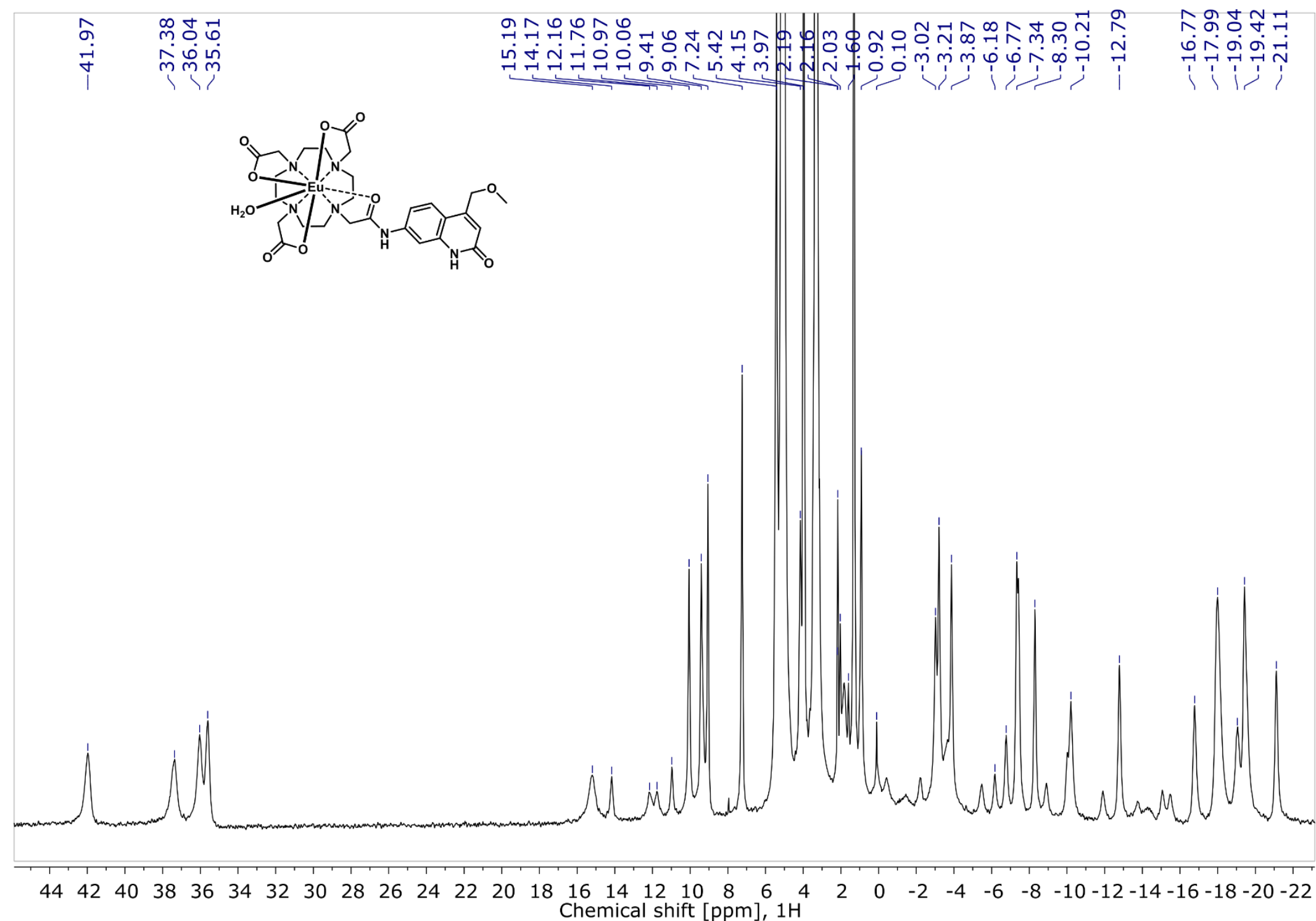

Figure S103. ${ }^{1} \mathrm{H}$ NMR spectrum of $\mathbf{E u L 1}^{\mathbf{M O M}}\left(400 \mathrm{MHz}, \mathrm{CD}_{3} \mathrm{OD}, 0{ }^{\circ} \mathrm{C}\right)$. 


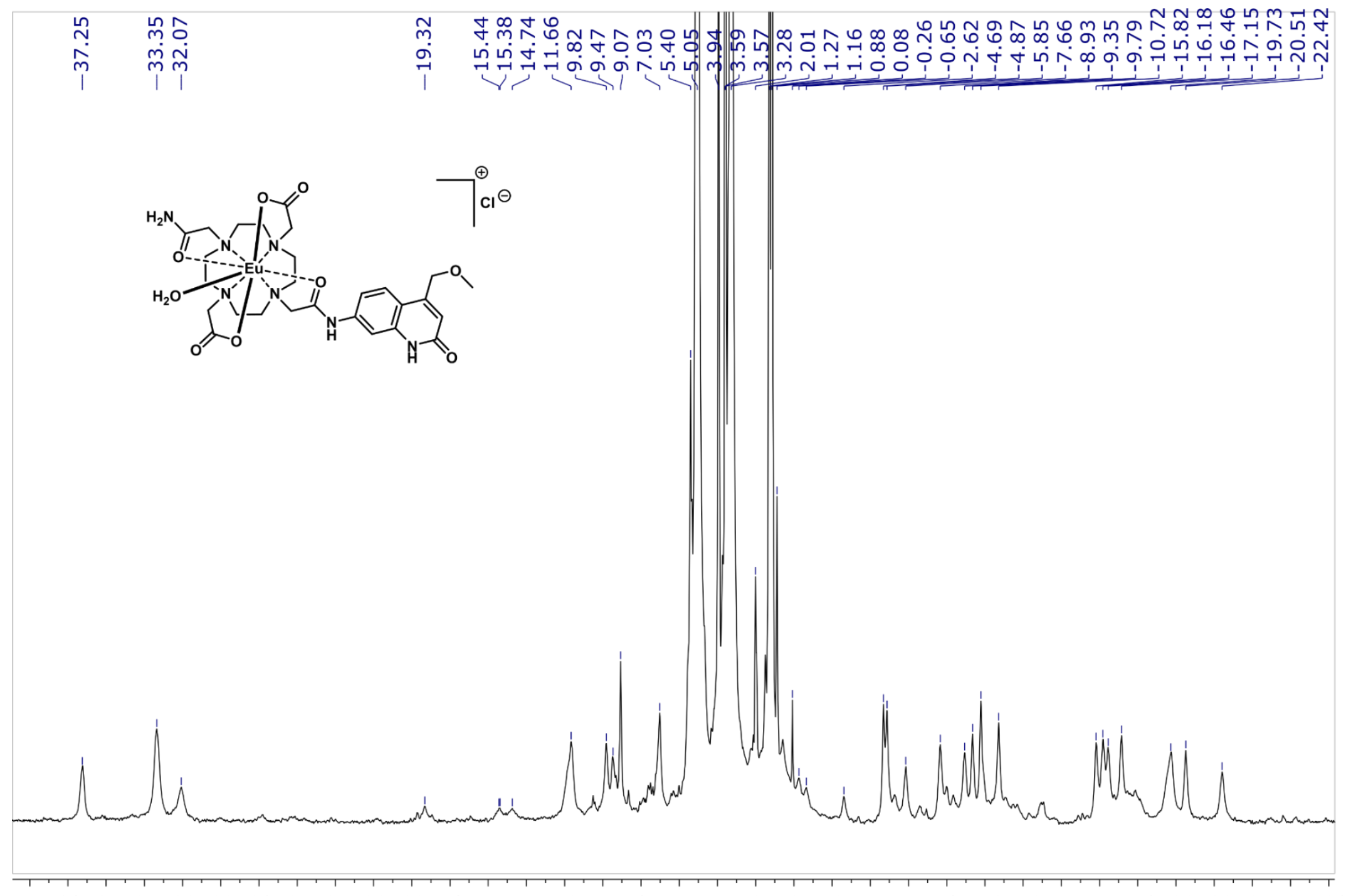

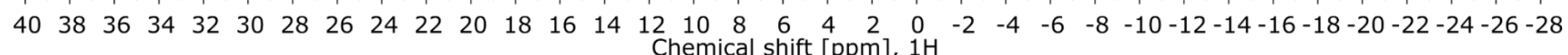

Figure S104. ${ }^{1} \mathrm{H}$ NMR spectrum of $\mathbf{E u L 2} 2^{\mathrm{MOM}}\left(400 \mathrm{MHz}, \mathrm{CD}_{3} \mathrm{OD}, 0{ }^{\circ} \mathrm{C}\right)$. 


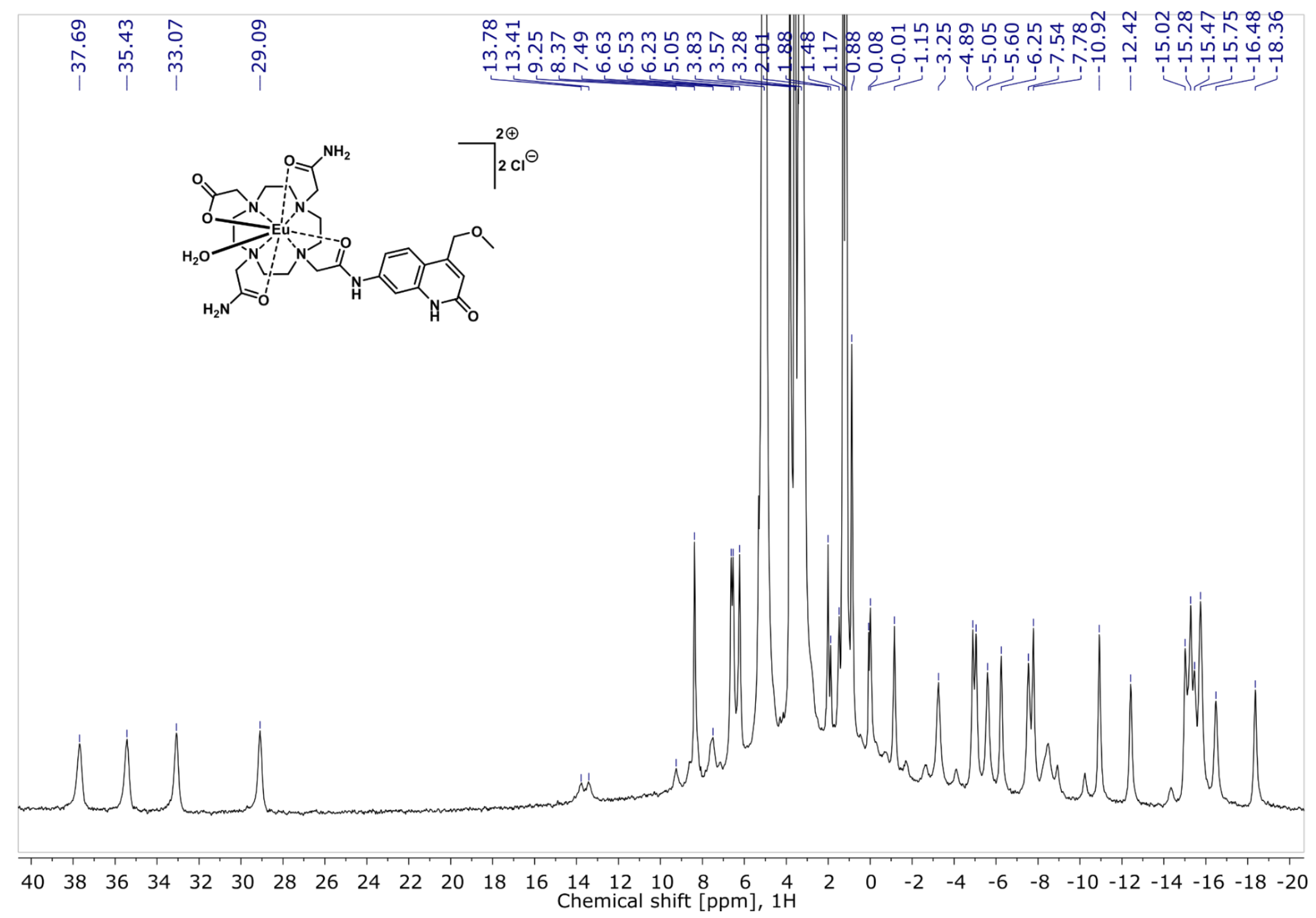

Figure S105. ${ }^{1} \mathrm{H}$ NMR spectrum of $\mathbf{E u L 3}^{\mathrm{MOM}}\left(400 \mathrm{MHz}, \mathrm{CD}_{3} \mathrm{OD}, 0{ }^{\circ} \mathrm{C}\right)$. 


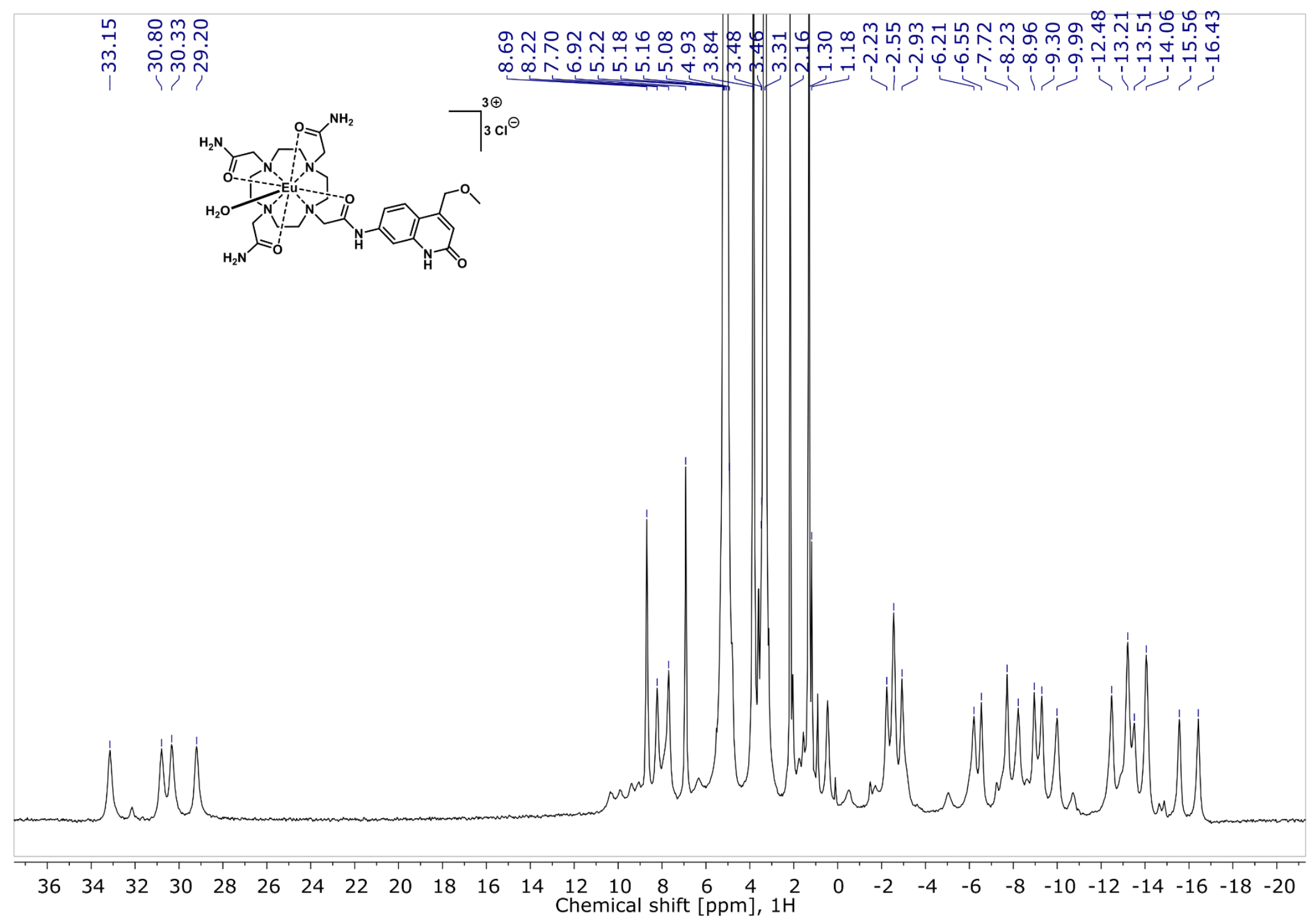

Figure S106. ${ }^{1} \mathrm{H}$ NMR spectrum of EuL4a ${ }^{\text {MOM }}\left(400 \mathrm{MHz}, \mathrm{CD}_{3} \mathrm{OD}, 0{ }^{\circ} \mathrm{C}\right)$. 


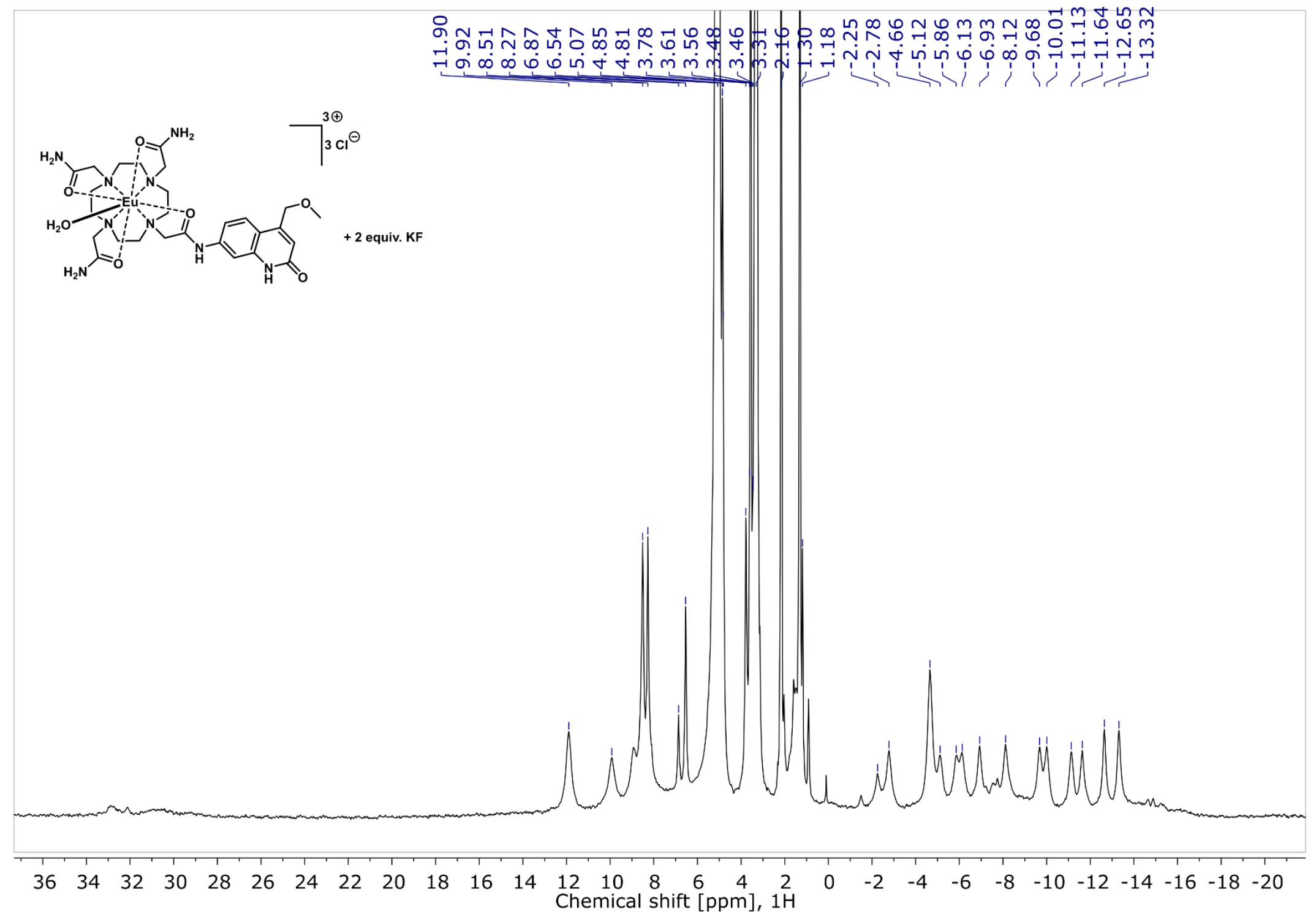

Figure S107. ${ }^{1} \mathrm{H}$ NMR spectrum of EuL4a ${ }^{\text {MOM }}$ with 2 equiv. $\mathrm{KF}\left(400 \mathrm{MHz}, \mathrm{CD}_{3} \mathrm{OD}, 0{ }^{\circ} \mathrm{C}\right)$. 


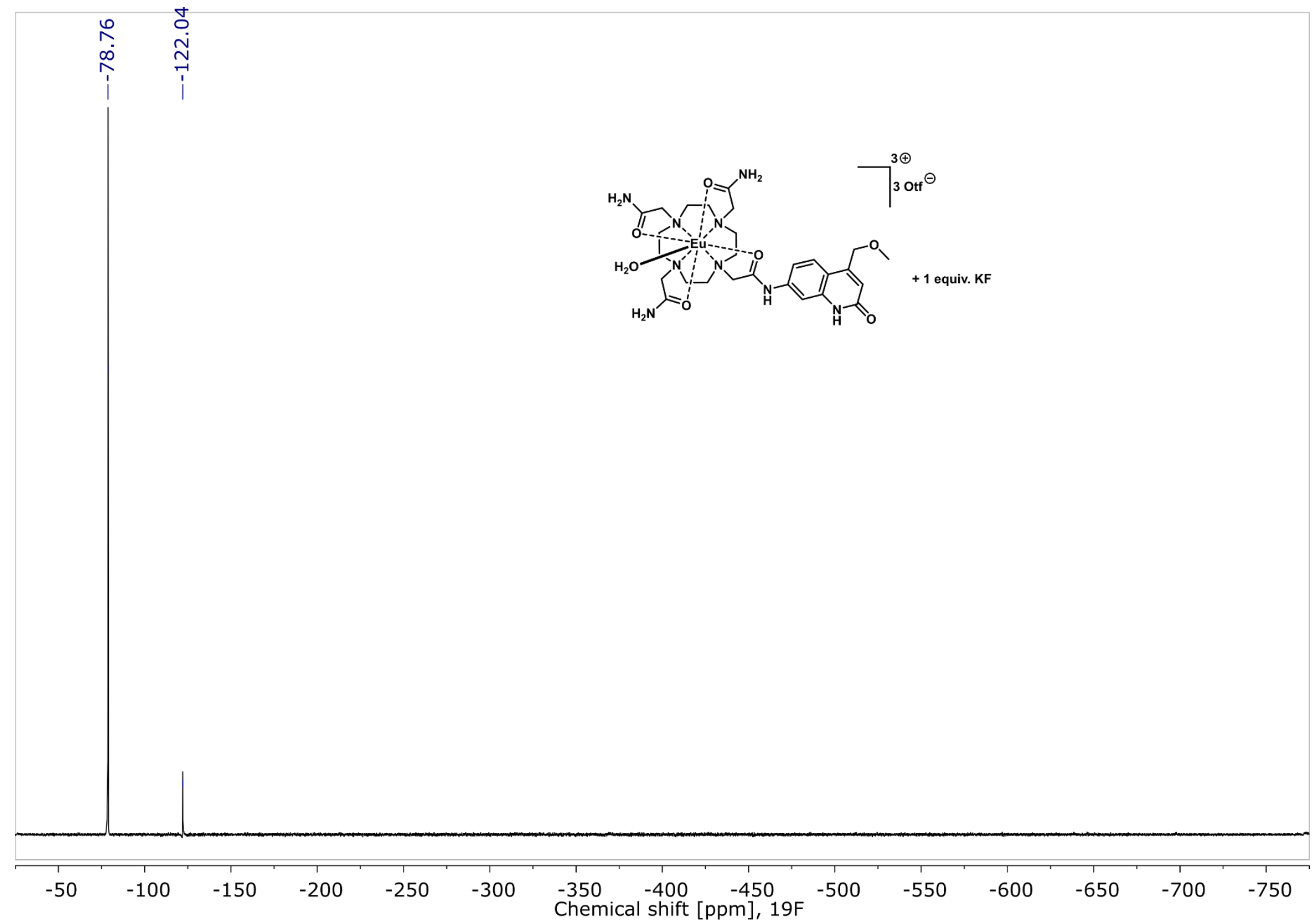

Figure S108. ${ }^{19} \mathrm{~F}$ NMR spectrum of EuL4a ${ }^{\text {MOM }}$ with 1 equiv. $\mathrm{KF}\left(376 \mathrm{MHz}, \mathrm{D}_{2} \mathrm{O}, 20{ }^{\circ} \mathrm{C}\right)$. 


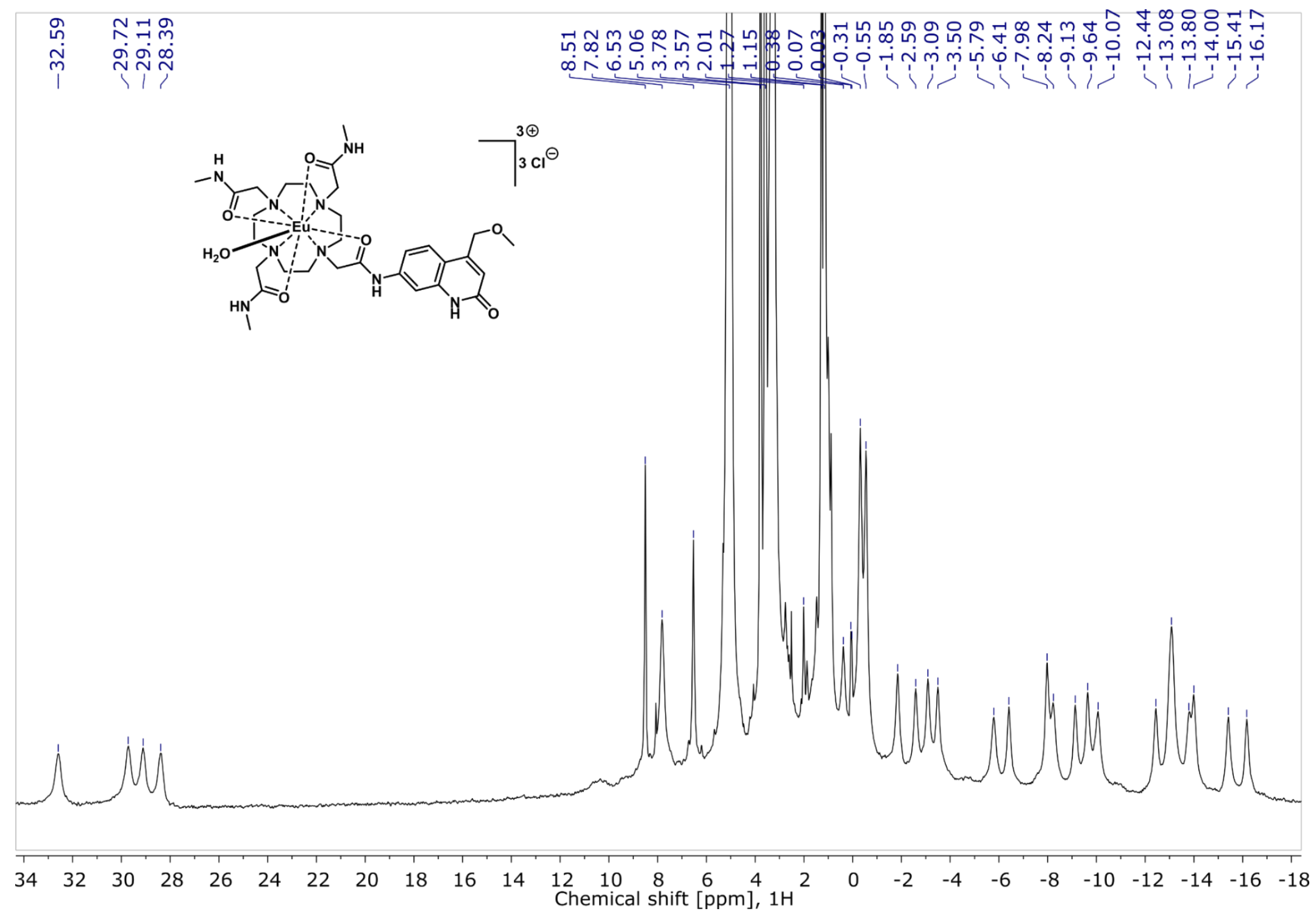

Figure S109. ${ }^{1} \mathrm{H}$ NMR spectrum of EuL4b ${ }^{\text {MOM }}\left(400 \mathrm{MHz}, \mathrm{CD}_{3} \mathrm{OD}, 0{ }^{\circ} \mathrm{C}\right)$. 


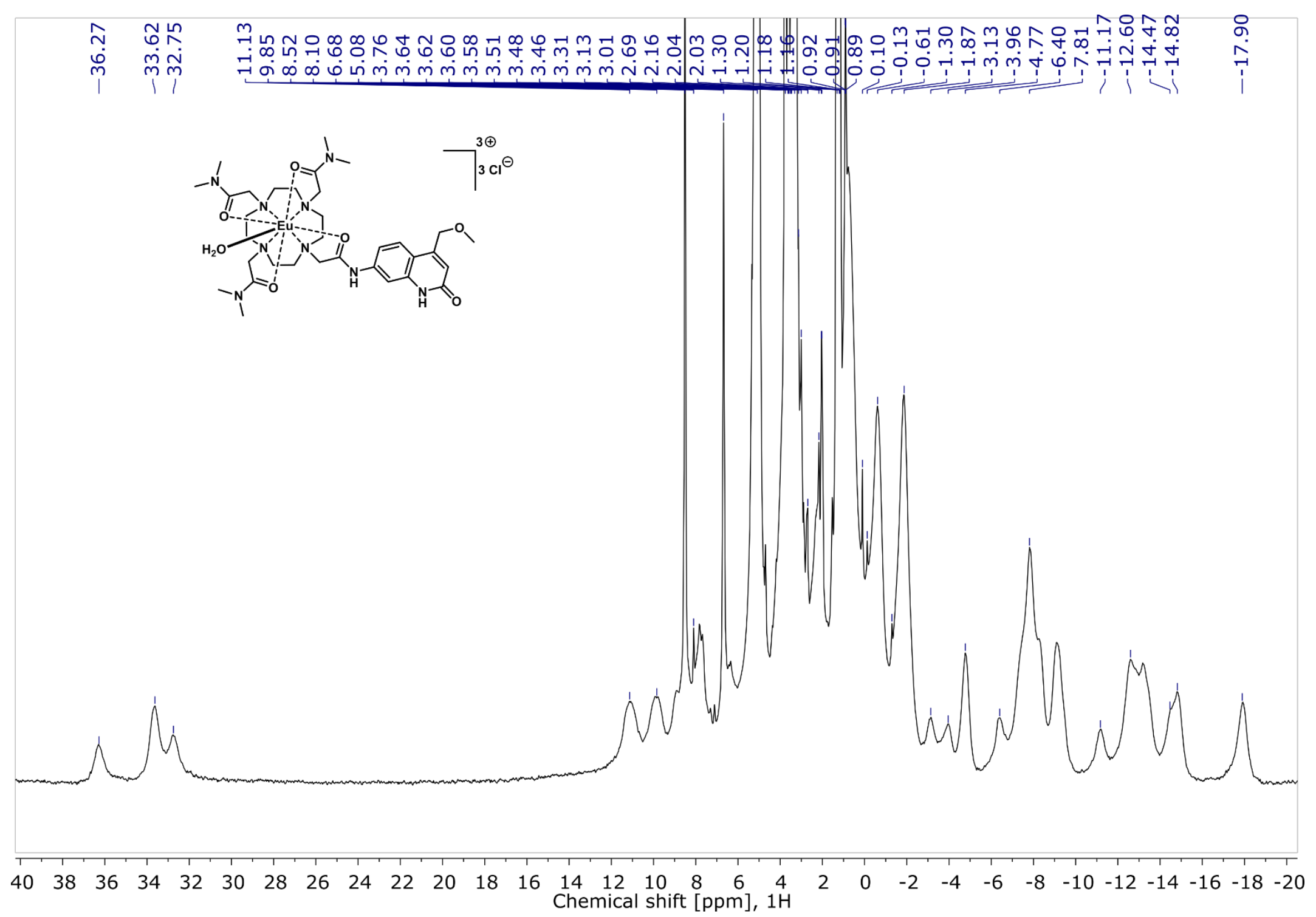

Figure S110. ${ }^{1} \mathrm{H}$ NMR spectrum of EuL4c ${ }^{\text {MOM }}\left(400 \mathrm{MHz}, \mathrm{CD}_{3} \mathrm{OD}, 0{ }^{\circ} \mathrm{C}\right)$. 


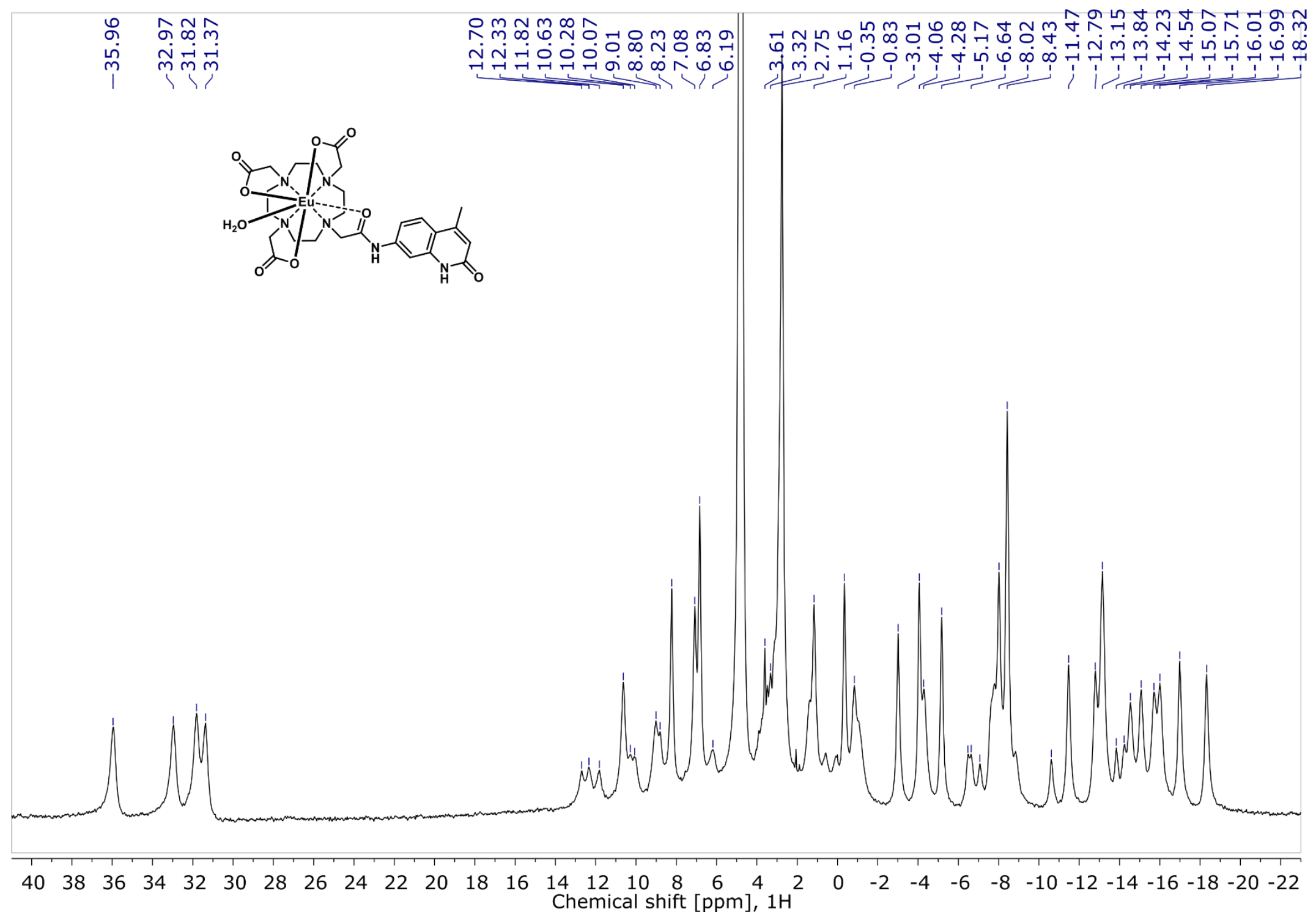

Figure S111. ${ }^{1} \mathrm{H}$ NMR spectrum of $\mathbf{E u L 1}^{\mathrm{Me}}\left(400 \mathrm{MHz}, \mathrm{D}_{2} \mathrm{O}, 10{ }^{\circ} \mathrm{C}\right)$. 


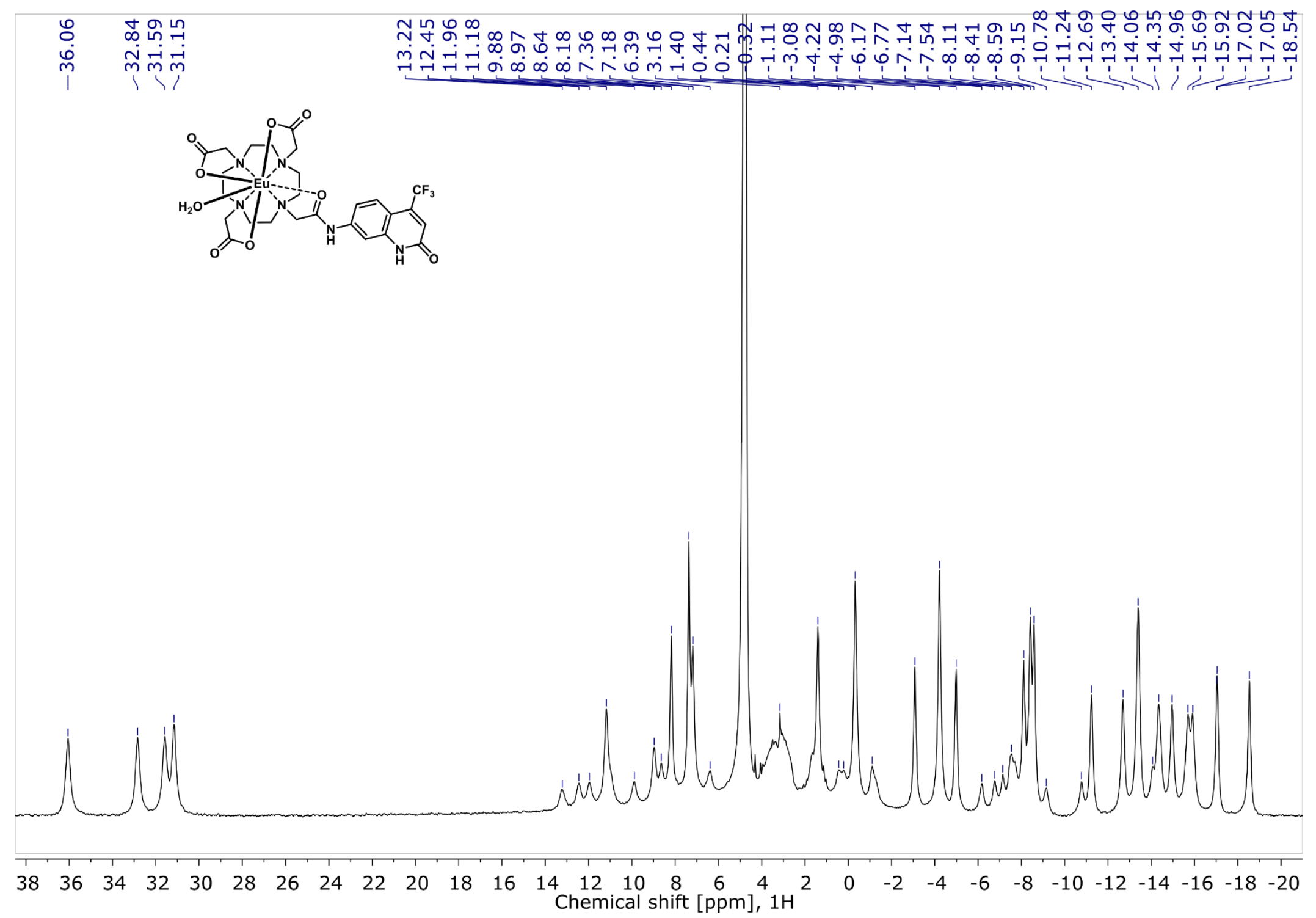

Figure S112. ${ }^{1} \mathrm{H}$ NMR spectrum of EuL1 ${ }^{\mathbf{C F} 3}\left(400 \mathrm{MHz}, \mathrm{D}_{2} \mathrm{O}, 10{ }^{\circ} \mathrm{C}\right)$. 


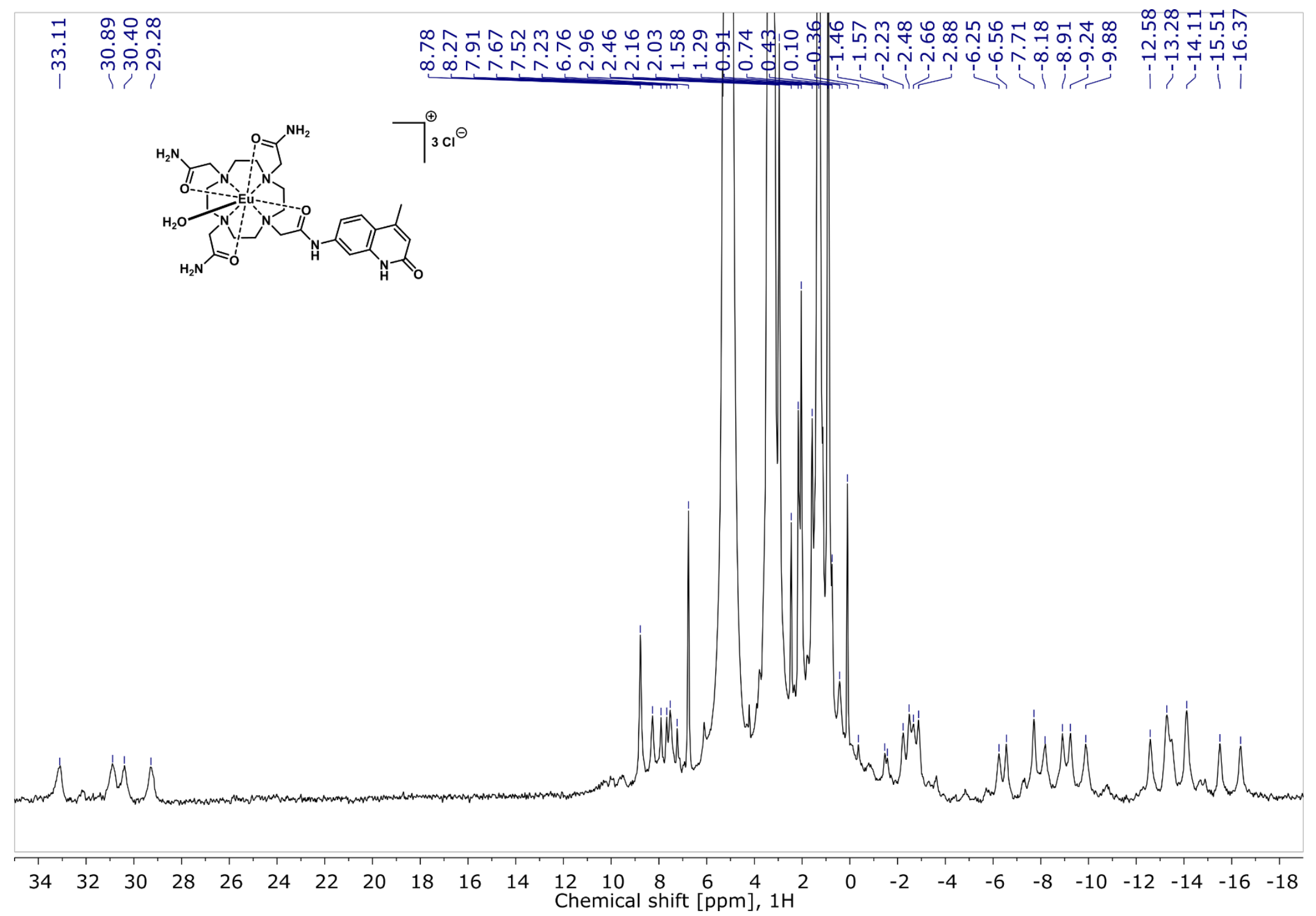

Figure S113. ${ }^{1} \mathrm{H}$ NMR spectrum of EuL4a ${ }^{\mathrm{Me}}\left(400 \mathrm{MHz}, \mathrm{CD}_{3} \mathrm{OD}, 0{ }^{\circ} \mathrm{C}\right)$. 


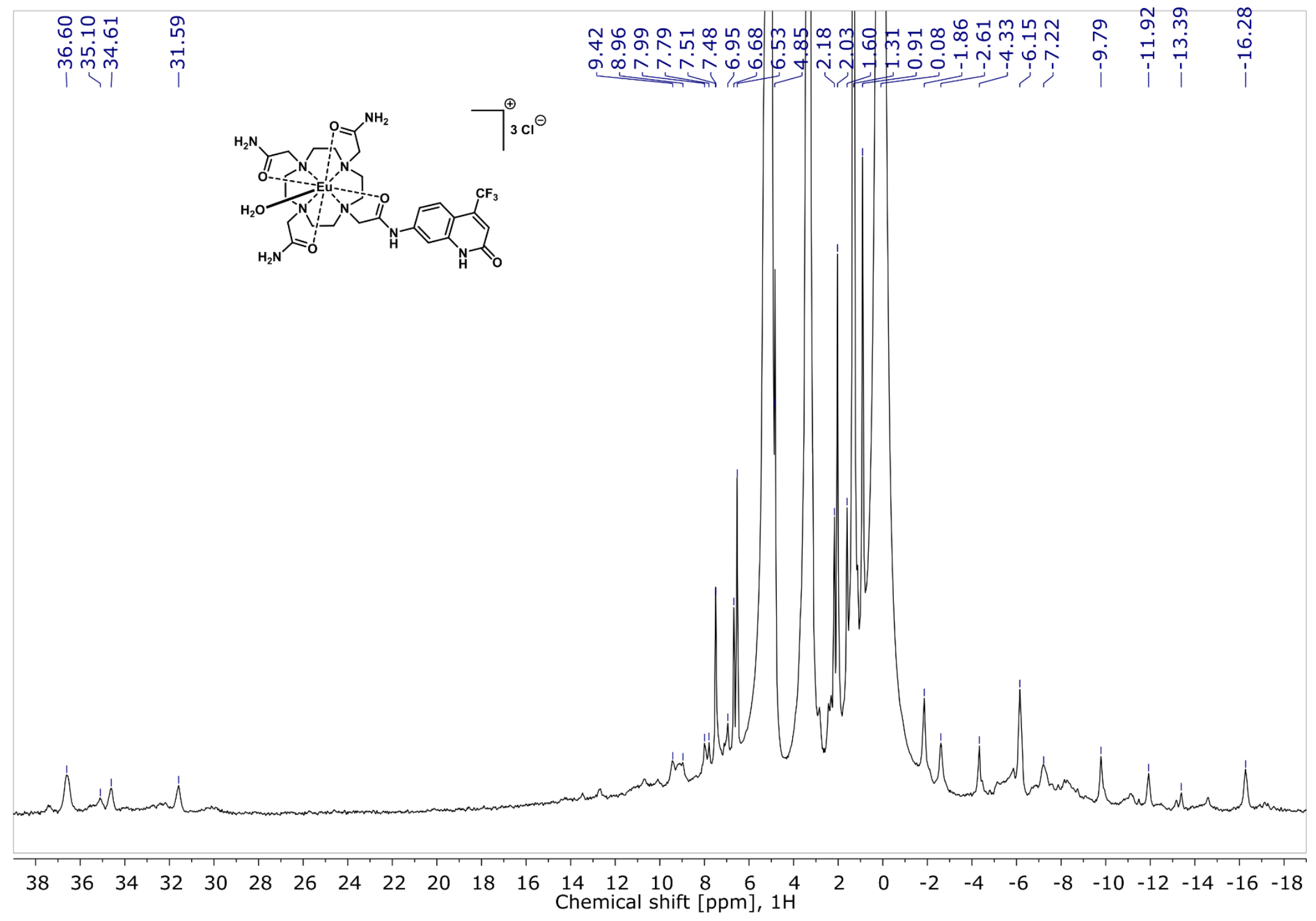

Figure S114. ${ }^{1} \mathrm{H}$ NMR spectrum of EuL4a ${ }^{\mathbf{C F 3}}$ (400 MHz, $\left.\mathrm{CD}_{3} \mathrm{OD}, 0{ }^{\circ} \mathrm{C}\right)$. 


\section{References}

1. Hopper, L. E.; Allen, M. J., Rapid synthesis of 1,7-bis(t-butoxycarbonylmethyl)-1,4,7,10tetraazacyclododecane (DO2A-t-Bu ester). Tetrahedron Lett. 2014, 55, 5560-5561.

2. León-Rodríguez, L. M. D.; Miranda-Olvera, A. D., Highly regioselective N-trans symmetrical diprotection of cyclen. Tetrahedron Lett. 2006, 47, 6937-6940.

3. Kovacs, D.; Lu, X.; Mészáros, L. S.; Ott, M.; Andres, J.; Borbas, K. E., Photophysics of Coumarin and Carbostyril-Sensitized Luminescent Lanthanide Complexes: Implications for Complex Design in Multiplex Detection. J. Am. Chem. Soc. 2017, 139, 5756-5767.

4. Suzuki, K.; Kobayashi, A.; Kaneko, S.; Takehira, K.; Yoshihara, T.; Ishida, H.; Shiina, Y.; Oishi, S.; Tobita, S., Reevaluation of absolute luminescence quantum yields of standard solutions using a spectrometer with an integrating sphere and a back-thinned CCD detector. Phys. Chem. Chem. Phys. 2009, 11, 9850-9860.

5. Supkowski, R. M.; Horrocks, W. D., Jr., On the determination of the number of water molecules, q, coordinated to europium(III) ions in solution from luminescence decay lifetimes. Inorg. Chim. Acta 2002, 340, 44-48.

6. Horrocks, W. D., Jr.; Sudnick, D. R., Lanthanide ion probes of structure in biology. Laser-induced luminescence decay constants provide a direct measure of the number of metal-coordinated water molecules. J. Am. Chem. Soc. 1979, 101, 334-40.

7. Beeby, A.; Clarkson, I. M.; Dickins, R. S.; Faulkner, S.; Parker, D.; Royle, L.; de, S. A. S.; Williams, J. A. G.; Woods, M., Non-radiative deactivation of the excited states of europium, terbium and ytterbium complexes by proximate energy-matched $\mathrm{OH}, \mathrm{NH}$ and $\mathrm{CH}$ oscillators: an improved luminescence method for establishing solution hydration states. J. Chem. Soc., Perkin Trans. 2 1999, 493-504.

8. Cramer, L. E.; Spears, K. G., Hydrogen bond strengths from solvent-dependent lifetimes of Rose Bengal dye. J. Am. Chem. Soc. 1978, 100, 221-227. 
9. Boens, N.; Qin, W.; Basarić, N.; Hofkens, J.; Ameloot, M.; Pouget, J.; Lefèvre, J.-P.; Valeur, B.; Gratton, E.; vandeVen, M.; Silva, N. D.; Engelborghs, Y.; Willaert, K.; Sillen, A.; Rumbles, G.; Phillips, D.; Visser, A. J. W. G.; van Hoek, A.; Lakowicz, J. R.; Malak, H.; Gryczynski, I.; Szabo, A. G.; Krajcarski, D. T.; Tamai, N.; Miura, A., Fluorescence Lifetime Standards for Time and Frequency Domain Fluorescence Spectroscopy. Anal. Chem. 2007, 79, 2137-2149.

10. Dickins, R. S.; Parker, D.; de Sousa, A. S.; Williams, J. A. G., Closely diffusing O-H, amide N-H and methylene $\mathrm{C}-\mathrm{H}$ oscillators quench the excited state of europium complexes in solution. Chem. Commun. 1996, 697-8.

11. Kovacs, D.; Phipps, D.; Orthaber, A.; Borbas, K. E., Highly luminescent lanthanide complexes sensitized by tertiary amide-linked carbostyril antennae. Dalton Trans. 2018, 47, 10702-10714.

12. Nielsen, L. G.; Sørensen, T. J., Including and Declaring Structural Fluctuations in the Study of Lanthanide(III) Coordination Chemistry in Solution. Inorg. Chem. 2020, 59, 94-105.

13. Kovacs, D.; Kiraev, S. R.; Phipps, D.; Orthaber, A.; Borbas, K. E., Eu(III) and Tb(III) Complexes of Octa- and Nonadentate Macrocyclic Ligands Carrying Azide, Alkyne, and Ester Reactive Groups. Inorg Chem 2020, 59, 106-117.

14. Odhner, H.; Jacobs, D. T., Refractive Index of Liquid D2O for Visible Wavelengths. J. Chem. Eng. Data 2012, 57, 166-168. 